\title{
INTERNATIONAL LINEAR COLLIDER
}

A TECHNICAL PROGRESS REPORT

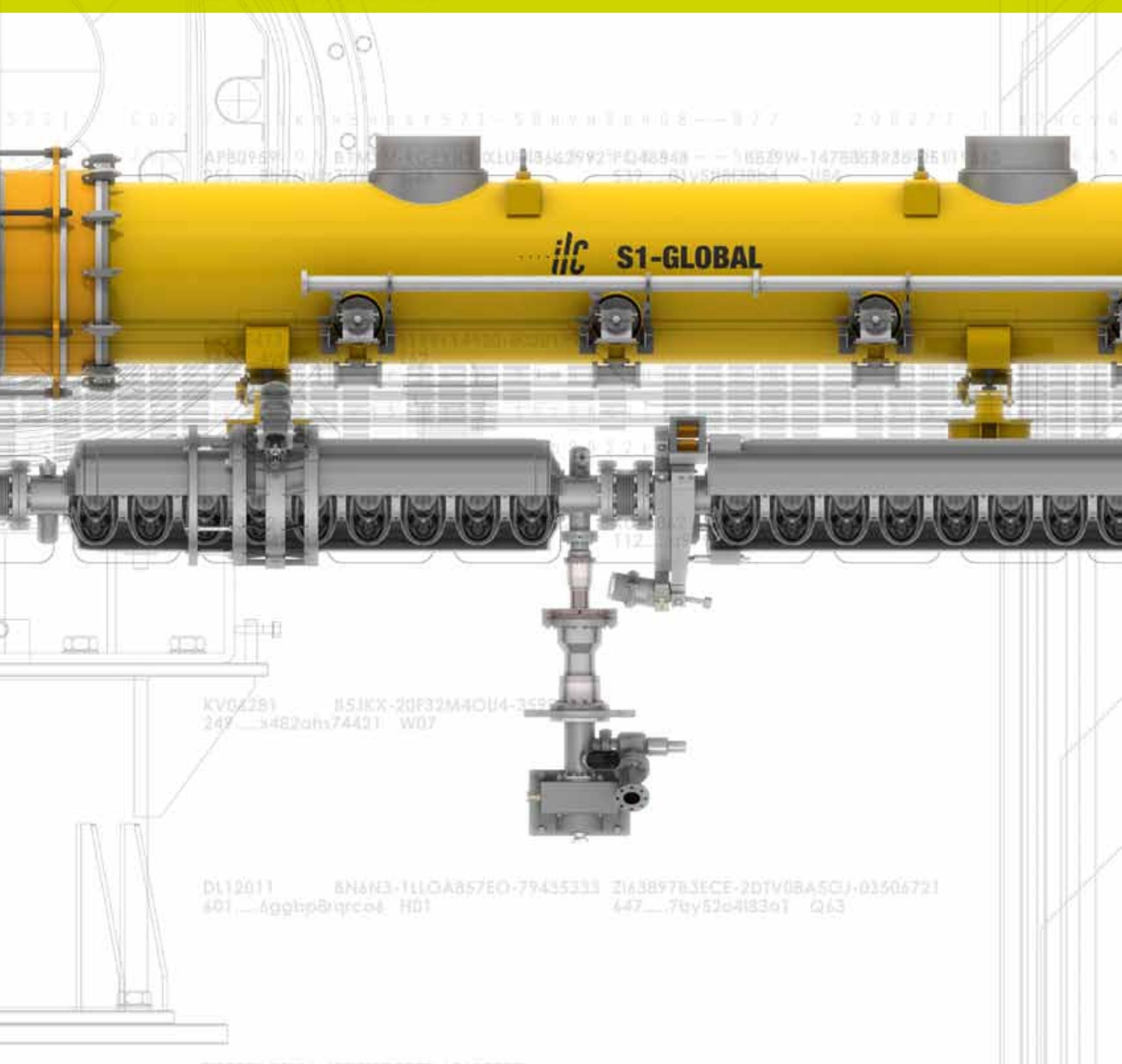




\section{DISCLAIMER}

This report was prepared as an account of work sponsored by an agency of the United States Government. Neither the United States Government nor any agency thereof, nor any of their employees, nor any of their contractors, subcontractors, or their employees, makes any warranty, express or implied, or assumes any legal liability or responsibility for the accuracy, completeness, or any third party's use or the results of such use of any information, apparatus, product, or process disclosed, or represents that its use would not infringe privately owned rights. Reference herein to any specific commercial product, process, or service by trade name, trademark, manufacturer, or otherwise, does not necessarily constitute or imply its endorsement, recommendation, or favoring by the United States Government or any agency thereof or its contractors or subcontractors. The views and opinions of authors expressed herein do not necessarily state or reflect those of the United States Government or any agency thereof.

The International Linear Collider: A Technical Progress Report marks the halfway point towards the Global Design Effort fulfilling its mandate to follow up the ILC Reference Design Report with a more optimised Technical Design Report (TDR) by the end of 2012. The TDR will be based on much of the work reported here and will contain all the elements needed to propose the ILC to collaborating governments, including a technical design and implementation plan that are realistic and have been better optimised for performance, cost and risk.

We are on track to develop detailed plans for the ILC, such that once results from the Large Hadron Collider (LHC) at CERN establish the main science goals and parameters of the next machine, we will be in good position to make a strong proposal for this new major global project in particle physics. The two overriding issues for the ILC R\&D programme are to demonstrate that the technical requirements for the accelerator are achievable with practical technologies, and that the ambitious physics goals can be addressed by realistic ILC detectors.

This GDE interim report documents the impressive progress on the accelerator technologies that can make the ILC a reality. It highlights results of the technological demonstrations that are giving the community increased confidence that we will be ready to proceed with an ILC project following the TDR. The companion detector and physics report document likewise demonstrates how detector designs can meet the ambitious and detailed physics goals set out by the ILC Steering Committee. LHC results will likely affect the requirements for the machine design and the detectors, and we are monitoring that very closely, intending to adapt our design as those results become available.

There are too many people involved in important ways in the ILC programme to thank them individually. Nevertheless, I would like to express our ongoing appreciation of the support of funding agencies around the world, and of the major high-energy laboratories for their continuing support. I also want to note the importance of our oversight committees, the International Committee for Future Accelerators and the ILC Steering Committee for their steadfast advice and scientific oversight, and of the Funding Agencies for Large Collaborations, both for their support of our common fund, and their ILC R\&D resource oversight.

Finally, as director of the GDE, I want to thank the dedicated group of accelerator scientists, engineers and technicians who continue to make such impressive progress in the face of the inevitable ups and downs of the overall project. This document is living proof!

BARRY BARISH, director, ILC Global Design Effort 


\begin{tabular}{|c|c|}
\hline EDITOR LIST & ECKHARD ELSEN \\
\hline & DESY, Germany \\
\hline & MIKE HARRISON \\
\hline & Brookhaven National Laboratory, USA \\
\hline & LEAH HESLA \\
\hline & Fermilab, USA \\
\hline & MARC Ross \\
\hline & Fermilab, USA \\
\hline & PERRINe Royole-DEgIEUX \\
\hline & CNRS/IN2P3, France \\
\hline & RIKA TAKAHASHI \\
\hline & KEK, Japan \\
\hline & NICHOLAS WALKER \\
\hline & DESY, Germany \\
\hline & BARBARA WARMBEIN \\
\hline & DESY, Germany \\
\hline & AKIRA YAMAMOTO \\
\hline & KEK, Japan \\
\hline & KAORU YOKOYA \\
\hline & KEK, Japan \\
\hline & MIN ZHANG \\
\hline & IHEP, China \\
\hline
\end{tabular}




\section{CONTENTS}

INTRODUCTION \& OVERVIEW 8

1.1 A global effort 9

1.2 Beyond the Reference Design Report: primary goals for the Technical Design Phase 11

1.3 The halfway point 12

1.4 Design process 13

1.5 Structure of the report 14

SUPERCONDUCTING RADIOFREQUENCY TECHNOLOGY 16

2.1 Putting superconducting radiofrequency technology to the test for the ILC 17

2.2 Development of worldwide infrastructure 22

2.3 Progress towards manufacture of high-gradient cavities 35

2.4 Cryomodule design and development

2.5 High-power radiofrequency development

2.6 System integrations tests

ACCELERATOR SYSTEM R\&D

3.1 The electron cloud R\&D programme

at CesrTA and other laboratories

3.2 The ATF2 final focus test beamline at KEK

ACCELERATOR DESIGN \& INTEGRATION

5.2 Life safety and infrastructure support

5.3 Site-specific design efforts 


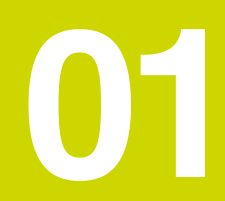

1.1 A GLOBAL EFFORT

1.2 BEYOND THE REFERENCE DESIGN REPORT:

PRIMARY GOALS FOR THE TECHNICAL DESIGN PHASE

1.3 THE HALFWAY POINT

1.4 DESIGN PROCESS

1.5 STRUCTURE OF THE REPORT

\section{INTRODUCTION \& OVERVIEW}


In 2003, more than 2,700 scientists from around the world signed a published statement Understanding Matter, Energy, Space and Time: the Case for the Linear Collider [1-1]. The statement made clear the undisputed worldwide consensus that the next energy frontier machine after the Large Hadron Collider (LHC) should be an electron-positron linear collider with an initial centre-of-mass energy reach of 500 gigaelectronvolts $(\mathrm{GeV})$, upgradable to 1 teraelectronvolt $(\mathrm{TeV})$. In the same year, the International Committee for Future Accelerators (ICFA) commissioned the International Technology Review Panel (ITRP) to select from the then competing acceleration technologies a single technology that would be the basis for an international design effort towards a truly global project. The following year, 2004, the ITRP recommended to ICFA that the 1.3-gigahertz superconducting radiofrequency (SCRF) technology developed by the TESLA collaboration [1-2] should form the basis for the design of the International Linear Collider (ILC).

\subsection{A GLOBAL EFFORT}

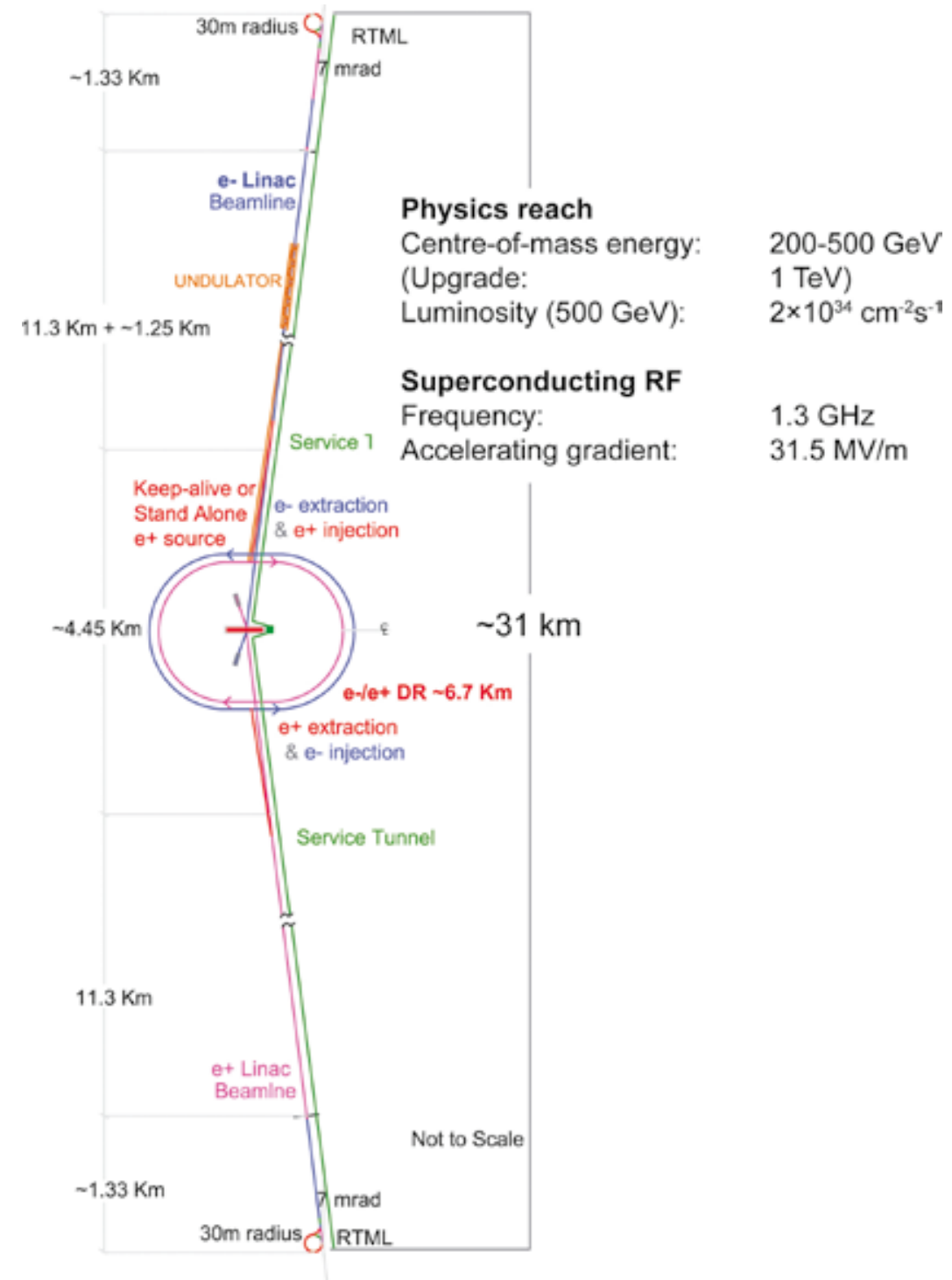

Figure 1.1. The layout of the ILC and its primary parameters as presented in the 2007 Reference Design Report. 
1 ILCU (ILC Unit) = 1 US 2007 \$

$(=0.83$ EUR $=117$ Yen $)$
Shortly after the ITRP decision, the International Linear Collider Steering Committee formally established the Global Design Effort (GDE) under the directorship of Barry Barish. Drawing on the resources of over 300 national laboratories, universities and institutes worldwide, the GDE produced the ILC Reference Design Report (RDR) [1-3] in August 2007. The report describes a conceptual design for the ILC reflecting an international consensus, together with an initial cost estimate of 6.70 billion ILCU ${ }^{1}$ for capital equipment costs, and 14,200 person-years of institutional labour. The layout and principal parameters of the design as presented in the RDR is shown in Figure 1.1.

The first two years of the GDE leading up to the publication of the RDR were focused on ILC design activities and the cost estimate. The work done during the RDR phase identified many high-risk challenges that required R\&D. The four highest-priority critical risk-mitigating R\&D areas were prioritised as:

1. SCRF cavities capable of reproducibly achieving at least 35 megavolts/ metre $(\mathrm{MV} / \mathrm{m})$.

2. A cryomodule consisting of eight or more cavities, operating at a gradient of $31.5 \mathrm{MV} / \mathrm{m}$.

3. Linac 'string test' (or integration test) of more than one cryomodule linac with beam.

4. Development of models and mitigation techniques for electron cloud effects in the positron damping ring.

Other R\&D areas (for example in the beam delivery system and the sources) were also identified, but were considered lower priority at the time of the RDR.

The first three priority R\&D items all relate to the superconducting radiofrequency linear accelerator technology, the primary cost driver of the machine. Although it was noted by the International Technology Review Panel that TESLA SCRF technology was 'mature', the ILC gradient goal had only been achieved in a handful of cavities (one of which had accelerated beam at $35 \mathrm{MV} / \mathrm{m}$ in the TESLA Test Facility at DESY - a proof of principle). Furthermore, the R\&D had been focused primarily in Europe centred at DESY, and a major goal for the GDE was to export this knowledge to the Americas and Asian regions. This had already begun during the RDR phase with the beginnings of development of the necessary SCRF infrastructure at Fermilab, Argonne National Laboratory and Jefferson Lab in the US, and KEK in Japan. Europe's own development has been driven by the design and construction of the European X-ray free-electron laser at DESY, which although independent of the GDE - uses very similar technology and can be considered a large prototype for the ILC.

This report concentrates on the work subsequent to the RDR and the priority R\&D identified therein. In 2007 the GDE began to restructure itself for an Engineering Design Phase, with the goal of producing an Engineering Design Report in 2010. Large funding cuts at the end of 2007 in the US and UK required the GDE management to rethink its strategy and timescale. With the loss of expected funding, it was clear that the originally planned engineering could not be done. Instead, it was decided to focus the remaining resources on the risk-mitigating $R \& D$. The programme was also extended by two years to 2012 and renamed to the Technical Design Phase. 
In 2008 the newly structured Global Design Effort technical project management published a five-year R\&D Plan that outlined the scope, milestones and goals of the Technical Design Phase. The R\&D Plan was intended to undergo regular revision and is now in its fifth version [1-4]. The plan clearly states the primary goals for the Technical Design Phase:

- results from critical R\&D programmes and test facilities that either demonstrate or support the choice of key parameters in the machine design;

- one or more models for a Project Implementation Plan, including scenarios for globally distributed mass-production of high-technology components as in-kind contributions;

- an updated and robust VALUE estimate and construction schedule consistent with the scope of the machine and the proposed Project Implementation Plan;

- an updated technical description of the ILC technical design in sufficient detail to justify the associated VALUE estimate.

The Technical Design Phase will culminate in the publication of a Technical Design Report (TDR) at the end of 2012. The TDR and its supporting documentation is intended to show a robust design and form a sound basis for a 'proposal to host' the project. The TDR is expected to build on the solid base of the RDR, but emphasise the Technical Design Phase activities towards the goals stated above:

- Results of critical R\&D. Unlike the RDR, which was primarily a design document, the TDR will focus on presenting the worldwide results of the technical R\&D during the five-year Technical Design Phase along with an updated design and cost.

- Project Implementation Plan. The plan has no counterpart in the RDR. It will deal with issues pertaining to the international project itself, such as governance, project structure, finance models and in-kind contributions. Of critical importance is the development of globally distributed massproduction models, particularly for the SCRF technology. These models will go beyond the simplistic ones assumed for the RDR, and will not only relate to how the machine will get constructed, but will also factor significantly into the cost estimate.

- Updated VALUE estimate. During the RDR phase a bottom-up cost estimate for all technical systems and components was made. This estimate was subsequently successfully defended to an international review. For the TDR, a complete reworking of the estimate is not expected. However it is considered mandatory to review and update the costs associated with the primary cost drivers: SCRF technology and conventional facilities, including civil construction. In particular for the SCRF, the new estimates will reflect the five years of R\&D in all three regions and must be consistent with the industrialisation models for globally distributed mass-production being developed. It is a stated goal of the Global Design Effort to make every attempt to produce a cost estimate in 2012 that does not significantly exceed the RDR estimate of 6.7 billion ILCU.

- Updated design. Again building on the solid base of the published 2007 Reference Design Report, an updated design will be presented in the TDR. This design will reflect not only the results of the risk-mitigating $\mathrm{R} \& \mathrm{D}$, but also many cost-driven design modifications resulting in a more cost-effective solution. An additional important aspect will be the
1.2 BEYOND THE REFERENCE DESIGN REPORT: PRIMARY GOALS FOR THE TeChinical Design PHASE 
further development of site-specific designs, taking into account the various differing constraints that individual sites may present. This is an important step forward from the RDR, which considered three conceptual generic sample sites from the standpoint of a single machine design. The resulting options will give potential hosts the flexibility in design needed to fulfil their specific site constraints.

The TDR will not be a complete engineered design and there will still be considerable engineering and design work to be done prior to start of construction. Identifying the scope of this remaining work - and the technical risk associated with it - is also an important task for the Technical Design Phase. However, the TDR will represent major technical progress over the $\mathrm{RDR}$, and present a significantly mature design, including significant progress on industrialisation for the SCRF technology and site-specific development.

\subsection{THE HALFWAY POINT}

This document, coming close to the mid-term of the TD Phase (or the end of 'Technical Design Phase 1'), is a major deliverable for the Global Design Effort. It represents a snapshot of the R\&D status: a large fraction of the R\&D projects continue through to the publication of the TDR in 2012, and in some cases beyond.

Highlights from the Phase 1 R\&D include:

- successful construction and commissioning, and/or further development of existing SCRF infrastructure at Fermilab, Argonne National Laboratory, Jefferson Lab and Cornell University in the US, KEK in Japan, and most recently the Institute of High Energy Physics, Chinese Academy of Sciences;

- identification of the preferred process for consistent production of $35-\mathrm{MV} / \mathrm{m}$ cavities (worldwide) and a successful demonstration of the Phase 1 goal of a 'production-like' yield of 50\% (towards the final R\&D goal of $90 \%$ for the TDR);

- as part of the above, and important for global mass-production, 'qualification' of new cavity vendors in the Americas and Asia to complement those already existing in Europe;

- construction and testing of more high-performance cryomodules (in particular at DESY as part of the preparation of the European X-ray FreeElectron Laser; this includes high-current beam tests at DESY's FLASH facility);

- international collaboration on the construction of a high-performance cryomodule at KEK, enabling plug-compatible design philosophies to be explored, as well as technology comparisons;

- start of developments towards SCRF mass-production models, including $R \& D$ for cost-effective industrialisation with an industrial R\&D pilot plant at KEK;

- reconfiguration of the Cornell Electron Storage Ring (CESR) at Cornell University in support of the CesrTA electron cloud programme, phase 1 of which was successfully concluded at the end of 2010 with a recommendation on electron cloud mitigation technologies for the ILC positron damping ring; 
- development of fast kicker technology for the damping rings, in particular tests in the Accelerator Test Facility (ATF) at KEK.

- Installation and commissioning of all subsystems and instrumentation of the final focus test beam at ATF2 at KEK;

- significant progress on the development of the machine-detector interface in support of the detector push-pull concept;

- progress on source R\&D, in particular on development of prototype polarised electron gun; demonstration of a prototype undulator section for the positron source; initialisation of engineering design and key R\&D on the positron target concepts and capture devices (ongoing).

In addition to the R\&D outlined above, the overall design of the ILC has been critically reviewed with respect to the identified cost drivers, and in particular conventional facilities and siting. As part of the stated need to constrain the costs, a primary goal was to reduce the underground volume required by the machine (the underground civil construction also represents one of the highest risks in terms of cost and schedule). After approximately 18 months of study, several design modifications to the 2007 published reference design were proposed, including:

- a single-tunnel solution for the main linear accelerators (linacs), which includes two novel concepts for radiofrequency power generation and distribution in support of that solution;

- a reduction in the number of bunches per pulse from 2625 to 1312 , facilitating cost savings via a reduction in the installed radiofrequency power and smaller damping rings, luminosity being in principle recovered by stronger focusing at the interaction point;

- moving the undulator-based positron production from the mid-point of the main electron linac (operating at a nominal $150 \mathrm{GeV}$ ) to the end, facilitating better integration into a consolidated 'central region' of the entire machine, which contains almost all the required systems other than the main linacs.

The above issues all have a direct impact on conventional facilities and siting. An accelerator design and integration team has been charged with developing a design based on these proposals. The goal is to achieve a consensus on the modified baseline early in 2011, which will then be used in Technical Design Phase 2 for further detailed design work and cost estimation. In order to facilitate a consensus-building mechanism, a Top-Level Change Control process was developed that focused on two workshops (Table 1.1). These workshops - open to all stakeholders and especially to the physics and detector community - have been used to carefully review the design modifications and finally submit a consensus proposal to the director. With the modified baseline agreed upon, the emphasis of the work for Technical Design Phase 2 will shift to consolidating that design and the ongoing R\&D towards producing the TDR in 2012. 
Table 1.1 Baseline Assessment Workshops (BAW) used for defining the modified ILC baseline for the Technical Design Report as part of the Top-Level Change Control process.

\begin{tabular}{|c|c|c|}
\hline BAW-I & September 2010, KEK [1-5] & $\begin{array}{l}\text { 1. Review of average accelerating gradient and } \\
\text { allowed gradient spread } \\
\text { 2. Single-tunnel option for main linacs and } \\
\text { associated novel RF power schemes }\end{array}$ \\
\hline BAW-II & January 2011, SLAC [1-6] & $\begin{array}{l}\text { 1. Relocation of the undulator-base positron } \\
\text { production to the end of the main electron linac. } \\
\text { 2. Reduction in bunch number and associated } \\
\text { modifications to the damping rings, main linac RF } \\
\text { and beam delivery system. }\end{array}$ \\
\hline
\end{tabular}

\subsection{STRUCTURE OF THE REPORT}

The remaining chapters of this report are intended to describe fully the Phase 1 achievements outlined above. Chapter 2 deals with the superconducting radiofrequency; chapter 3 deals with accelerator systems R\&D (i.e., non-SCRF R\&D); chapter 4 discusses the important work associated with the conventional facilities and siting (the second primary cost driver), and in particular the site-specific issues; chapter 5 discusses briefly the accelerator design and integration work and the updated baseline and parameters; and finally chapter 6 looks towards the remaining work and the publication of the TDR.

\section{References}

[1-1] http://physics.uoregon.edu/ lc/wwstudy/

Ic_consensus.html

[1-2] TESLA TDR: http://lcdev.kek.jp/TESLA-TDR/

[1-3] RDR: http://www.linearcollider.org/about/

Publications/Reference-Design-Report
[1-4] TDP R\&D Plan: http://ilc-edmsdirect.desy.de/ ilc-edmsdirect/file.jsp?edmsid $={ }^{\star} 813385$

[1-5] BAW-I indico site: http://ilcagenda.linearcollider. org/internalPage. py?pageld=5\&confld=4593
[1-6] BAW-II indico site: http://ilcagenda.linearcollider. org/conferenceDisplay.py?confld=4612 
INTRODUCTION \& OVERVIEW 


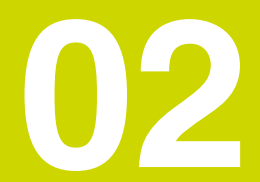

2.1 PUTTING SUPERCONDUCTING

RADIOFREQUENCY TECHNOLOGY

TO THE TEST FOR THE ILC

2.2 DEVELOPMENT OF WORLDWIDE INFRASTRUCTURE

2.3 PROGRESS TOWARDS

REPRODUCIBLE MANUFACTURE OF

HIGH-GRADIENT CAVITIES

2.4 CRYOMODULE DESIGN AND DEVELOPMENT

2.5 HIGH-POWER RADIOFREQUENCY DEVELOPMENT

2.6 SYSTEM INTEGRATIONS TESTS

\section{SUPERCONDUCTING RADIOFREQUENCY TECHNOLOGY}




\subsubsection{SCRF development in the Technical Design Phase}

$\mathrm{R} \& \mathrm{D}$ for superconducting radiofrequency (SCRF) technology is a very high-priority and global technical activity for the ILC in the Technical Design Phase (TDP) [2-1, 2, 3]. This activity builds on and extends the pioneering work done in the decade or so leading up to the International Technology Review Panel's choice of SCRF technology in 2004 [2-4]. In that decade, R\&D on 1.3-gigahertz $(\mathrm{GHz})$ technology done by the TESLA Technology Collaboration succeeded in reducing the cost per megaelectronvolt $(\mathrm{MeV})$ by a large factor over the early 1990 s state-of-the-art SCRF. The original goal was to reduce costs by increasing the operating accelerating gradient by a factor of five from 5 megavolts per metre $(\mathrm{MV} / \mathrm{m})$ to $25 \mathrm{MV} / \mathrm{m}$, and reducing the cost per metre of the complete accelerating module by a factor of four for large-scale production [2-1-5].

The pace of progress has continued during the TDP, with the state-of-the-art cavity performance now exceeding $40 \mathrm{MV} / \mathrm{m}$ in the vertical low-power test, approaching the physical critical field limit for niobium.

During the first half of the TDP, under the prioritised R\&D guidance set by the Global Design Effort (GDE), the baseline cavity processing recipe was improved and optimised in most labs participating in the so-called So study programme (see section 2.3) to develop high-gradient superconducting cavities. Jefferson Laboratory (JLab) in the US reports that they have achieved repeatable cavity processing for cavities that can reach a gradient of more than $35 \mathrm{MV} / \mathrm{m}$ consistently. Argonne National Laboratory (ANL), Fermilab and KEK have started to demonstrate high-quality processing and cavity results. It is anticipated that a reliable process will be demonstrated in labs worldwide by the end of the TDP. This effort, along with the transfer of the processing technology to industry in all three regions, will put ILC cavity processing on a strong footing by the end of the TDP.

In the last three years, some 60 cavities were built for the purpose of demonstrating the ILC gradient. Of these, more than half were subjected to the defined standard process, while the others were used for developing the process or qualifying the equipment in use. The fraction of cavities manufactured using the standard process that meet or exceed the performance criteria can be used to estimate construction project performance (generally referred to as production yield).

Advancement in SCRF cavity performance has followed two tracks: the control of field emission typically caused by mechanical defects or contaminant particles and the reduction of localised heating caused by non-uniformities located near the cavity equator welds.

Major progress in both tracks was made before 2007, including the use of high-purity niobium material, the introduction of high-pressure water rinsing, and the development of electropolishing. Quality control and quality assurance in the production of the niobium starting sheet and in the fabrication of complete cavities, such as sheet scanning, have been introduced by DESY as well as by some other labs.

\subsection{PUTTING}

SUPERCONDUCTING

RADIOFREQUENCY

TECHNOLOGY TO THE

TEST FOR THE ILC 
In view of this progress, a forward-looking gradient goal of $35 \mathrm{MV} / \mathrm{m}$ or higher in the low-power vertical test with 90\% production yield was adopted for the reference design. This remains the primary goal to be demonstrated during the TDP.

Field emission is a major cause of variability in cavity performance. After the GDE's SCRF team had formed in 2007, it took the first step to suppress field emission following advice from the TESLA Technology Collaboration to introduce rinses intended to clear away the debris left behind by the electropolishing process [2-6, 7]. This has proved quite effective and the incidence of strong field emission has been greatly reduced after ethanol and degreaser chemical rinses were introduced.

The development of surface diagnostic techniques capable of quantitative evaluation of surface defects has proceeded in parallel. The most important of these is the development of the high-resolution 'Kyoto-KEK' camera. The camera can be precisely positioned inside the cavity and provides images of the electropolished mirror-like niobium surfaces that can be accurately referenced to external features.

A long-term goal of the cavity optical inspection development is to enable cavity performance predictions based on observed features prior to cavity RF testing at cryogenic temperatures. To meet this goal, a definitive correlation between camera image characteristics and external temperature-mapping measurements made during low-power tests as well as cavity quench behaviours need to be established. This work has just begun and already shows promising results; as a result of this $R \& D$, we are now able to conclude that all observed hot spots that cause severe gradient limits (i.e., a gradient below $20 \mathrm{MV} / \mathrm{m}$ ) have a corresponding internal surface defect. However, the converse does not appear to be true, that is, not all observed surface blemishes limit cavity performance. It is possible that more subtle details in the observed features control the quench behaviour. Optical inspection at higher resolution and in three dimensions is being explored at various labs to settle the issue. Further work on this topic will continue throughout TD Phase 2 and beyond; in particular, the mass production of the European-XFEL cavities will provide a large and valuable optical inspection dataset.

\subsubsection{Main linac SCRF operational performance}

Nowhere is the increased pace of progress more evident than in the expansion of global test infrastructures and in the fostering of cavity fabrication companies in each region. Halfway through the four-year Technical Design Phase, four institutional cavity process and test facilities (one in Europe, two in the Americas and one in Asia), are actively providing ILC cavities fabricated by four companies (two in Europe, one in the Americas and one in Asia). We expect to roughly double the number of qualified cavity fabrication vendors in the remaining half of the Technical Design Phase. Fully functional hightechnology cavity production capability in each region is mandatory for providing the ILC project with a strong global technology basis. 
In addition to fabrication and processing sites, the last few years have also seen the development of cryomodule assembly and SCRF linac beam test facility infrastructures in each region. These infrastructures make up the link between individual cavity testing and full linac performance. Following qualification in the low-power vertical test, cavities are connected in a string to allow the passage of the beam and are provided with coaxial power couplers. The string is placed in the 12-m-long cryomodules, which are in turn joined together in the accelerator enclosure tunnel. To characterise the performance of the finished linac complex we test the assembled cryomodules and groups of cryomodules with beam in test linac facilities. The GDE has established qualification performance criteria for each step of the cryomodule production through to a final linac system performance specification (average beam accelerating gradient). The criteria are summarised in Table 2.1 [2-8].

\begin{tabular}{|c|c|c|c|}
\hline $\begin{array}{l}\text { Cost-relevant design } \\
\text { parameter(s) for TDR }\end{array}$ & $\begin{array}{l}\text { ILC main linac cavity } \\
\text { operational specification }\end{array}$ & $\begin{array}{l}\text { R\&D goal for cavity gradient } \\
\text { in vertical test }\end{array}$ & $\begin{array}{l}\text { Table } 2.1 \text { Cavity performance specification } \\
\text { and R\&D goals. }\end{array}$ \\
\hline $\begin{array}{l}\text { Gradient in vertical test, } \\
\text { including the } 2 \text { nd pass }{ }^{1}\end{array}$ & $\begin{array}{l}35 \mathrm{MV} / \mathrm{m} \text { at } \mathrm{Q}_{0} \geq 8 \times 10^{9} \text {, average } \\
\text { with spread } \leq \pm 20 \%\end{array}$ & $\begin{array}{l}35 \mathrm{MV} / \mathrm{m} \text { at } 90 \% \text { yield, } \\
\text { equivalent to } \geq 38 \mathrm{MV} / \mathrm{m} \text {, average }\end{array}$ & \\
\hline $\begin{array}{l}\text { Cavity-string gradient in } \\
\text { cryomodule test }\end{array}$ & $34 \mathrm{MV} / \mathrm{m}$, average & & \\
\hline $\begin{array}{l}\text { Main linac operational } \\
\text { gradient }\end{array}$ & $\begin{array}{l}31.5 \mathrm{MV} / \mathrm{m} \text { at } \mathrm{Q}_{0} \geq 1 \times 10^{10} \\
\text { average, with spread } \leq \pm 20 \%\end{array}$ & & ${ }^{1}$ Second-pass refers to a second surface process \\
\hline
\end{tabular}

In the table, both the ILC main linac operational specification and the R\&D goal are described in terms of an average cavity gradient to be achieved with an allowance for peak-to-peak gradient spread. Cavity performance is listed for two test stages: a vertical low-power test of individual cavities and a high(pulsed-) power cryomodule test, after a cavity has been connected to a cavity string and inserted into a cryostat. The main linac operational gradient refers to the gradient at which the cavity can operate indefinitely following installation in the main linac. The table lists an R\&D goal of not more than $3 \%$ deterioration of cavity gradient from vertical test to cryomodule test, assuming the $35 \mathrm{MV} / \mathrm{m}$ with the $90 \%$ yield to $34 \mathrm{MV} / \mathrm{m}$ on average, respectively. It also lists an R\&D goal operational limit of not more than 1.5 MV/m below the limit seen in the cryomodule test and an operational controls margin gradient of not more than $3 \%$.

The construction of ILC SCRF linac beam test facilities in each of the three regions is the largest investment in new SCRF infrastructure. These projects (section 2.6) will be complete or will be nearing completion by the end of the Technical Design Phase. These cornerstone facilities, listed in Table 2.2, complete the SCRF high-technology capability that provides the global basis for the ILC. In each case, the facility serves to advance regional projects, which are only indirectly related to ILC. This adds resources and strength and diversifies the development effort. Five cryomodules based on the ILC technology, along with 80 very similar cryomodules for the European XFEL, will be constructed during the period leading up to 2014. This includes the 'S1-Global' cryomodule recently built and tested at KEK with cavities contributed by institutions in each region (section 2.4). 
Table 2.2 Regional ILC SCRF technology

development and testing centres.
Linac beam test centres

(beam on date)

ILCTA-NML (2012)

Quantum-Beam/STF-2

(2011/2013)

CEA-Irfu/CNRS-LAL/DESY for FLASH (from 2005)

FLASH and E-XFEL

Table 2.2 summarises the number of cavity fabrication companies that are either qualified or expected to be qualified during the TDP, the institutional process and low-power testing centres that have throughput capability of dozens of cavities per year and the cryomodule assembly and high-power testing centres and linac beam test facilities, with expected initial operation date.

\subsubsection{Challenges for SCRF technology}

Underpinning the overall strategy of the SCRF R\&D plan is the desire to produce the best possible cost-optimised solution for the main linac using state-of-the-art technology. Good progress has been made in TDP-1 towards these goals, and they remain fundamentally unchanged in TDP-2, which also sees a shift in emphasis towards the development of industrial massproduction models, (section 2.6). The milestones for the TD Phase SCRF goals outlined are summarised in Table 2.3 [2-1].

\begin{tabular}{|c|c|c|c|}
\hline Stage & Subjects & Milestones to be achieved & Year \\
\hline so & Nine-cell cavity & $\begin{array}{l}35 \mathrm{MV} / \mathrm{m} \text {, maximum, at } \mathrm{Q}_{0} \geq 8 \times 10^{9} \text {, with a production } \\
\text { yield of } 50 \% \text { in TD Phase } 1 \text {, and } 90 \% \text { in TD Phase } 2\end{array}$ & $\begin{array}{l}2010 / \\
2012\end{array}$ \\
\hline S1 & Cavity-string & $\begin{array}{l}31.5 \mathrm{MV} / \mathrm{m} \text {, average, at } \mathrm{Q}_{0} \geq 10^{10} \text {, in one cryomodule, } \\
\text { including a global effort, and } 34 \mathrm{MV} / \mathrm{m} \text {, average, in TD } \\
\text { Phase } 2\end{array}$ & $\begin{array}{l}2010 / \\
2012\end{array}$ \\
\hline S2 & $\begin{array}{l}\text { Cryomodule- } \\
\text { string }\end{array}$ & $\begin{array}{l}31.5 \mathrm{MV} / \mathrm{m} \text {, average, with full beam loading and } \\
\text { acceleration }\end{array}$ & 2012 \\
\hline
\end{tabular}


The interim TDP-1 R\&D milestone of cavity production yield of more than $50 \%$ at $35 \mathrm{MV} / \mathrm{m}$ (including cavities which have undergone a second surface treatment and test process) has been achieved (section 2.3). We consider strategies for gradient improvement, categorising and prioritising tasks leading to the Technical Design Report. These are:

Short-term R\&D goals:

- manufacturing process including quality control.

- preparation with surface treatment including a time- and cost-effective process.

- further studies towards the understanding and (further) reduction of field emission.

Long-term R\&D goals:

- cost-effective mass production technology such as seamless hydroforming, large-grain niobium with chemical polishing.

- further fundamental $R \& D$ to reach much higher gradient to be adaptable for a future upgrade towards $1 \mathrm{TeV}$, such as alternative cavity shapes.

For the latter, we expect to capitalise on the tremendous progress and investment of the last few years. In the coming months, we will outline the cost saving and performance-enhancing strategies to be implemented using the new infrastructure. 
2.2 DeVelopMent

OF WORLDWIDE

INFRASTRUCTURE

\subsubsection{Development of infrastructure at KEK, in Asia}

The Asia region's effort for SCRF activity and infrastructure is centred at the Superconducting RF Test Facility (STF) at KEK [2-9]. It includes facilities for cavity surface process, cavity and cavity string assembly in cleanrooms, cryomodule assembly, cavity performance tests in the vertical position and cryomodule tests in the horizontal position in an undergrounded tunnel. The general layout is shown in Figure 2.1. Further efforts for new SCRF infrastructures have also progressed in laboratories in China and India.
Figure 2.1 General layout of the Superconducting RF Test Facility at KEK.

\section{STF (Superconducting RF Test Facility)}

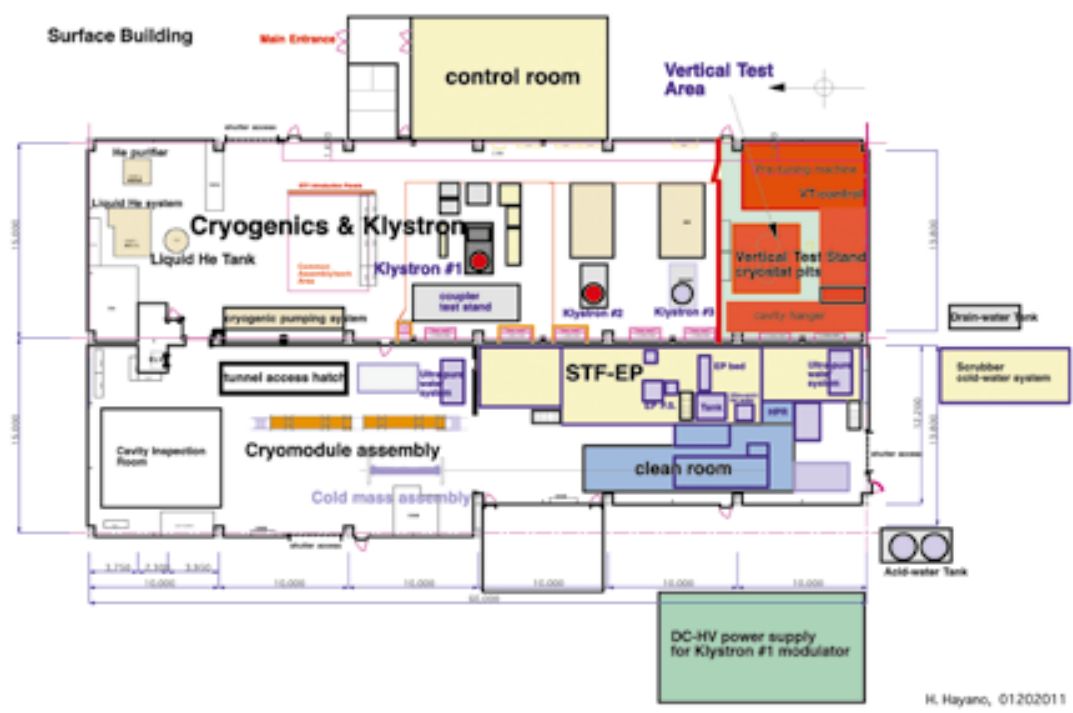

For the cavity process and treatment at STF, a new electropolishing (EP) facility was constructed and became operational in 2008 [2-10]. The system includes the EP system, buffered chemical polishing utilities for small parts and flange surface etching, ultrasonic rinsing, a high-pressure rinsing system and a cleanroom for cavity assembly. Optimisation of the EP and high-pressure rinsing system has been a major continuing subject following initial commissioning. Various diagnostics are provided to monitor and record every EP treatment, the EP process itself and subsequent water rinsing in order to control the temperature to stay below $35^{\circ} \mathrm{C}$. A specific study examined the optimisation of the EP current density by setting a reasonably low current density (30 to 35 milliamperes $(\mathrm{mA})$ per square centimetre $\left(\mathrm{cm}^{2}\right)$ ) to suppress sulphur generation. The ultrasonic rinse solution was tested to ensure removal of precipitated sulphur particles from the cavity surface. As a result of the rinsing investigation, a $2 \%$ solution of commercially available FM-20 detergent has become a regular part of the process. Effluent water from the high-pressure rinse is routinely monitored by using a particle counter and a total organic component device for monitoring contamination. Figure 2.2 shows the EP facility established at KEK during TD Phase 1. 


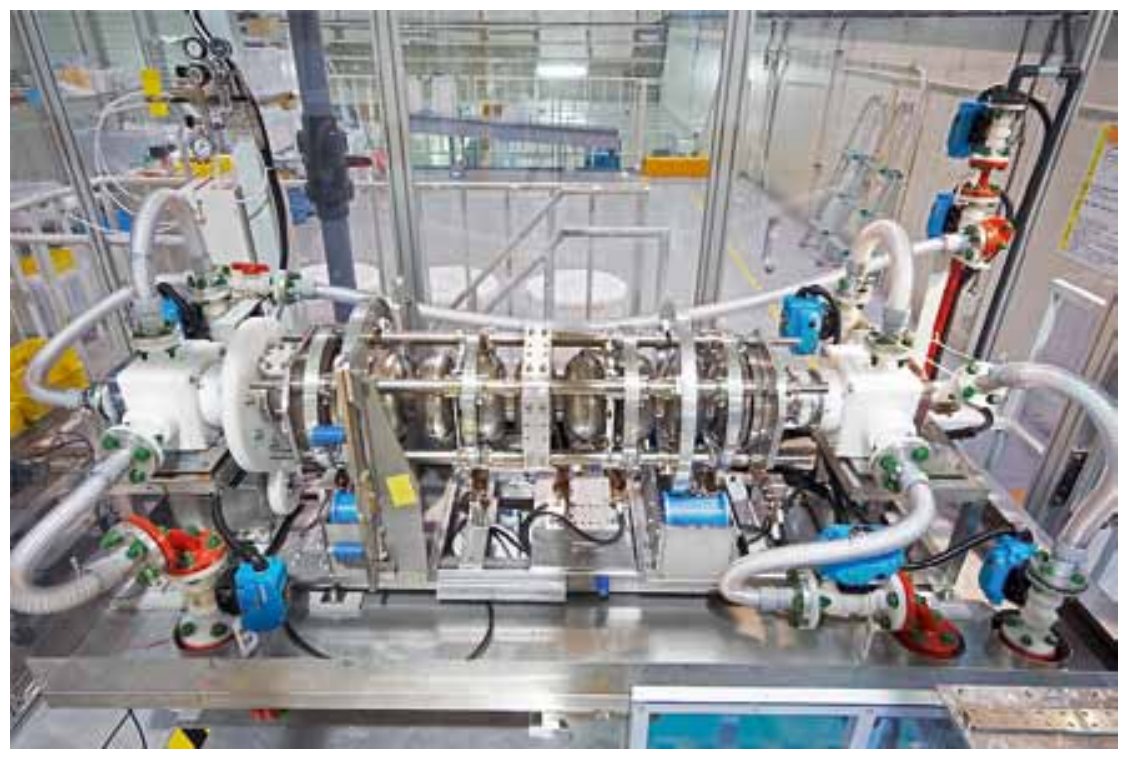

Figure 2.2 The EP facility established at KEK-STF.

The vertical test stand for low-power cavity field testing was also commissioned in 2008. For the vertical test, the first 4-m-deep cryostat was installed in STF, assembled together with radiation shielding and helium pumping system. The test stand is now routinely operated once per week. It uses a 2,0oo-litre liquid helium supply with dewars for the cooling and cold test. Figure 2.3 shows the vertical test facility at KEK-STF.

A monitoring system for full temperature and X-ray mapping is used for every vertical cavity test [2-11]. A total of 352 carbon-resistor temperature sensors and 142 PIN diode X-ray sensors are attached around each cell and end group higher order mode (HOM) coupler. Observed temperature rise and $\mathrm{X}$-ray intensity data are summarised by a 'map plot' in real time in order to easily identify heat spots and to facilitate the connection with the surface inspection that follows each test. 
Figure 2.3 The vertical test stand established at KEK-STF.

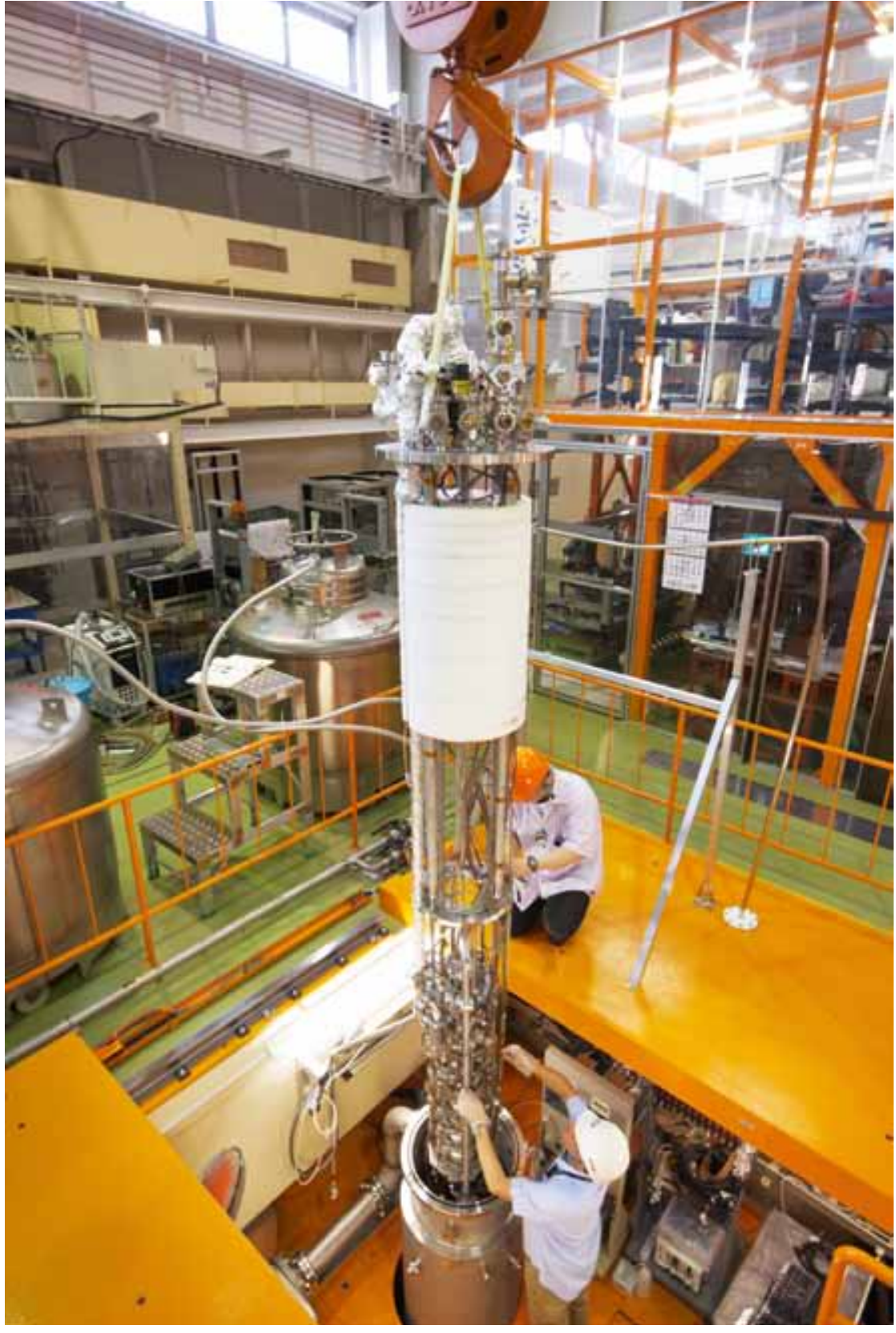

The development and subsequent upgrade of a cavity surface inspection camera system was done in collaboration with KEK and Kyoto University [2-12]. A newly developed high-performance complementary metal-oxide semiconductor (CMOS) camera combined with an LED illumination system resulted in a highly improved resolution, a ten-times increase in brightness and much longer-life illumination. Automated image capturing and defectfinding software was developed and is to be further optimised. Figure 2.4 shows the cavity surface inspection camera system, together with an example of a high-resolution image of the electron-beam weld. 



Figure 2.4 Left: Kyoto-KEK optical inspection camera system. Right: an example of automated image capture software.

In case a cavity has clearly visible defects inside and its performance is limited by these defects, a surface repair tool is useful. A miniature grinding mechanism with very small charge-coupled device (CCD) observation camera that fits within the 78-mm diameter of the cavity has been developed as shown in Figure 2.4 [2-13]. Recently, this device, combined with a technique to get precise internal defect information using a replica-mould technique, has been successfully used to repair a cavity at STF. One good example is the nine-cell cavity MHI-08. A pit-type defect $(0.7 \mathrm{~mm}$ by $0.5 \mathrm{~mm}$ in size and about 115 micrometres $(\mu \mathrm{m})$ in depth) was found using the inspection camera at precisely the $16 \mathrm{MV} / \mathrm{m}$ quench location indicated by the thermal monitor system. After grinding by using a local grinding machine developed at KEK (Figure 2.5) and ensuing 50- $\mu \mathrm{m}$ surface removal by EP, the cavity gradient performance was improved to $27 \mathrm{MV} / \mathrm{m}$ with no observation of heating at that location. After two additional EP cycles, a gradient of $38 \mathrm{MV} / \mathrm{m}$ was achieved in the fourth vertical test.

The installation of an automated cavity tuning machine is now operational at STF. It was originally developed at DESY and two additional sets have been fabricated in cooperation with DESY and Fermilab, as described later. The machine has the capability of field flatness tuning and cell-to-cell alignment correction through linked operation between the automated software, beadpull, six-jaw cell deforming, and cavity eccentric measurement system. 
Figure 2.5 Local grinding tool with an expandable motor stage installed in a 50-mm-diameter cylindrical housing.

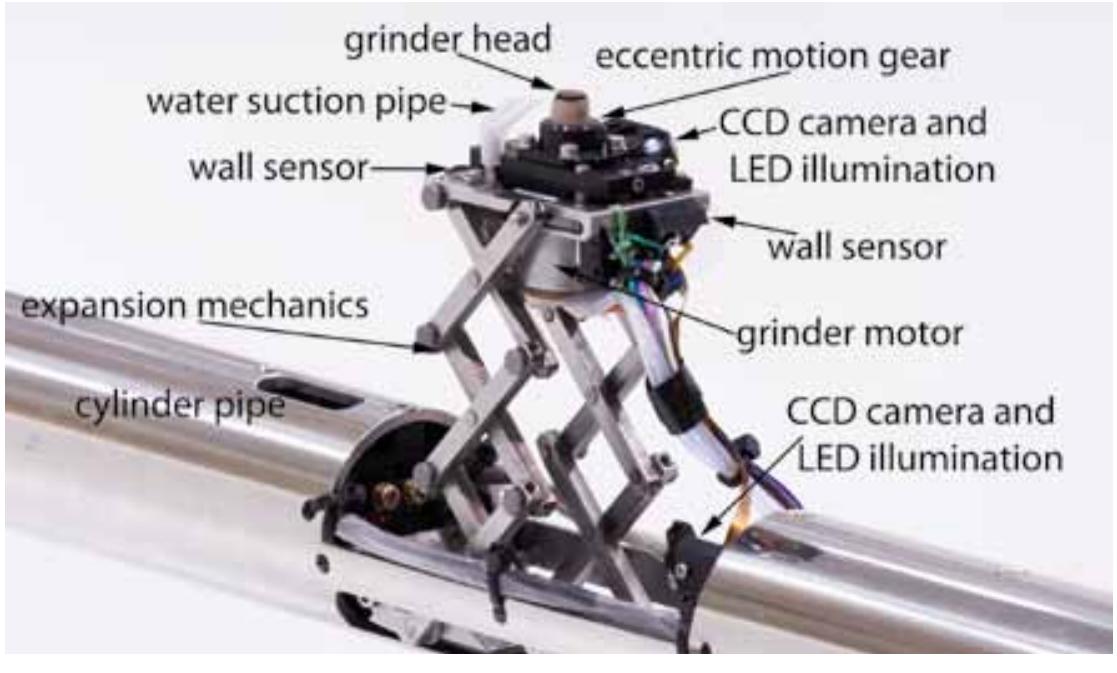

Figure 2.6 General layout of the cavity fabrication facility in progress at KEK.
The development of a cavity fabrication facility (a pilot plant) is in progress at KEK to study cost-effective fabrication technology. To prepare for industrialisation, various R\&D efforts are required, along with the initiative of researchers of the laboratories and in close cooperation with industry. The facility at KEK includes an electron beam welder, a trimming machine, a press machine and a chemical pre-process facility. Figure 2.6 shows the general layout. The press machine and the trimming machine were already installed and commissioned in 2010. The electron beam welder is to be delivered to KEK in early 2011 . The cavity fabrication study for forming cells and end group components was started. In order to simplify the machining steps, the use of a pressing technology for various fabrication processes is now under investigation. The close cooperation with industrial partners is much encouraged in these R\&D efforts. The cavities to be fabricated by using the facility are expected to be installed into cryomodules and tested at STF between 2013 and 2014 .






\subsubsection{Development of infrastructure in the Americas}

Infrastructure development in the Americas has included a focus on new industrial capabilities to add to existing ones and the development of new diagnostic and test facilities at laboratories [2-13, 14, 15, 16, 17, 18, 19, 20, 21]. Nearly 100 nine-cell cavities sourced from industry in both Europe and the United States have been procured through the Americas regional team for the purposes of improving both infrastructure at the laboratories and capabilities in industry. The typical process for developing a vendor includes production and test of several single-cell prototypes and, after successful testing of these, progression to production of full nine-cell cavities. At the time of the RDR there was one vendor in the United States qualified to build nine-cell cavities; as of this writing not only has that vendor tuned up their production process to successfully make multiple nine-cell cavities, but a second vendor has recently had its first nine-cell cavity tested. It reached a gradient of $29 \mathrm{MV} / \mathrm{m}$ with no field emission. In addition, single cell cavities have been tested from a third Americas region vendor, and we have processed and tested single cell cavities fabricated in India as well.

In parallel with the cavity effort, processing facilities were developed at ANL and Fermilab, based strongly on the existing capabilities at JLab to develop the throughput capacity for both the cavities required for cryomodules and those being used for R\&D. Figure 2.7 shows the general layout of the ANL/ Fermilab surface processing facility located at ANL [2-22].




This has included the creation of EP and high-pressure rinsing systems and associated rooms at ANL that are now complete. The ongoing installation and commissioning of furnaces at Fermilab and Cornell removes the last large bottleneck in the production of cavities and allows capacity for further R\&D. To operate the ANL/Fermilab facility, technicians were located and trained at JLab for extended periods. Throughout this effort, the processing and test of cavities at JLab has continued, and the performance of nine-cell cavities coming from JLab has been consistently excellent. The engineering of this processing facility, and the standardisation of the chemical processing steps, has now led to a chemical polishing facility being assembled in industry.

In 2007 the vertical test system at Fermilab was commissioned, and since has been used at an increasing rate such that two cool-downs per week are now possible, for either nine-cell or single-cell tests [2-23]. A unique facility in the Americas, the Horizontal Test Facility at Fermilab, used for the testing of dressed cavities, was brought online in 2008 and has been used since at a rate of approximately one test per month to qualify the cavity dressing process before assembly into an ILC-style cryomodule, known as CM-2 [2-16]. Figure 2.8 shows the Horizontal Test Facility at Fermilab.

Figure 2.8 The Horizontal Test Facility at Fermilab.



Production, test and diagnostic facilities at the laboratories in the Americas region have been enhanced not only by propagation of tools developed elsewhere, such as the Kyoto-KEK camera system, but also through local efforts such as the creation of the controls system for the automatic tuning machine (since delivered to DESY and KEK and deployed at Fermilab), second sound quench detection location development, Questar optical inspection system development, automation and software development of the optical inspection process, development of a silicone moulding technique for the $3-\mathrm{D}$ imaging of surface features, development and commissioning of a vertical EP facility and creation of a fast thermometry system for measurements of quench location and hot spots. Industrial X-ray tomography has been used to view voids in the cavity material, typically around the equator welds, that currently appear to be a leading indicator of fabrication difficulties that 
could lead to quench limitations during tests. With respect to remediation efforts, laser re-melting has been shown to smooth single-cell cavity surfaces successfully and to be minimally invasive, while tumbling techniques are under continued study both as a remediation and a preventive measure. Figure 2.9 shows the Fermilab tumbling process facility.

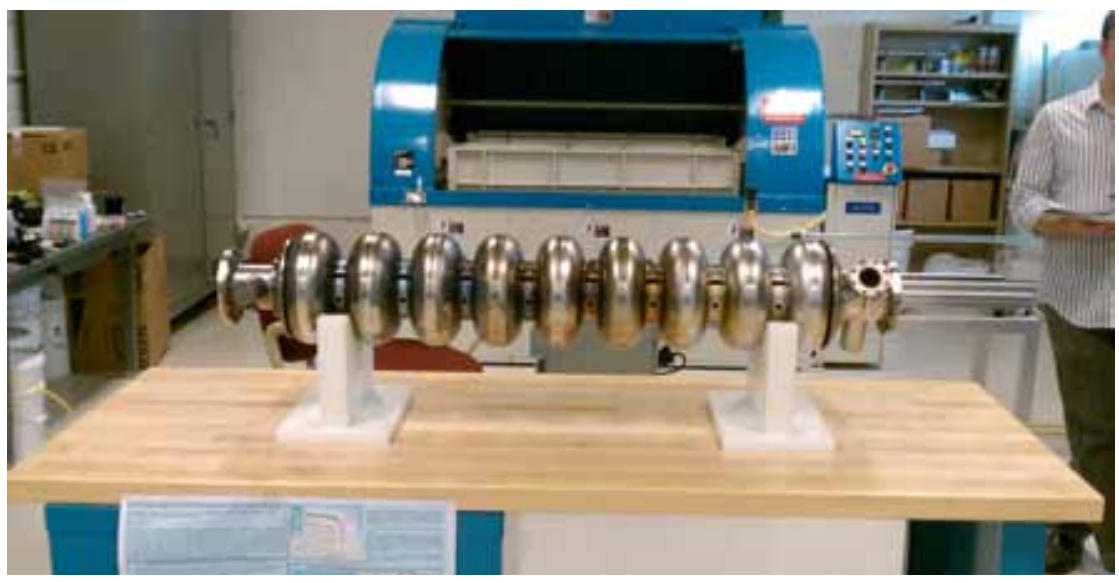

In addition to cavity fabrication and processing, Fermilab hosts a full cryomodule assembly facility capable of a throughput of one cryomodule per month. Figure 2.10 shows the cryomodule assembly facility at Fermilab. The design is strongly based on the system at DESY and has been used to date to assemble Cryomodule 1 (CM-1), and to dress multiple cavities currently undergoing testing in the horizontal test stand in preparation for CM-2 assembly.

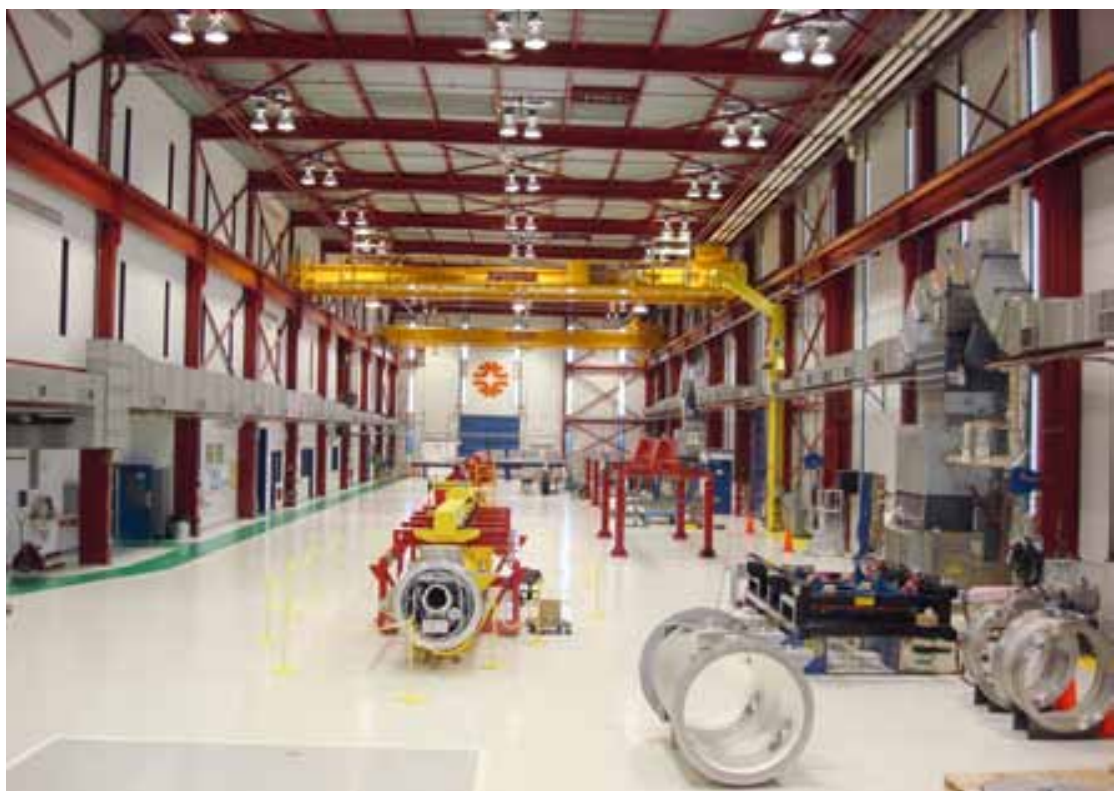


Leading up to the completion of the TD phase, the Americas region has additional vertical test stand and horizontal test facilities on order to increase capacity, and should be well positioned to process and test tens of cavities, finally assembling them into cryomodules in 2011 and beyond. Our diagnostic and test capabilities will continue to be used to diagnose faults earlier and earlier in the fabrication and processing chain, and to feed information back to our vendors.

Fermilab is currently constructing the SCRF Test Accelerator at the New Muon Lab (NML). NML consists of a photo-emitted RF electron gun, followed by a bunch compressor, low-energy test beamlines, SCRF accelerating structures, and high-energy test beamlines. Figure 2.11 shows the general layout of NML. The progress of the system integration and tests is discussed in section 2.6 .

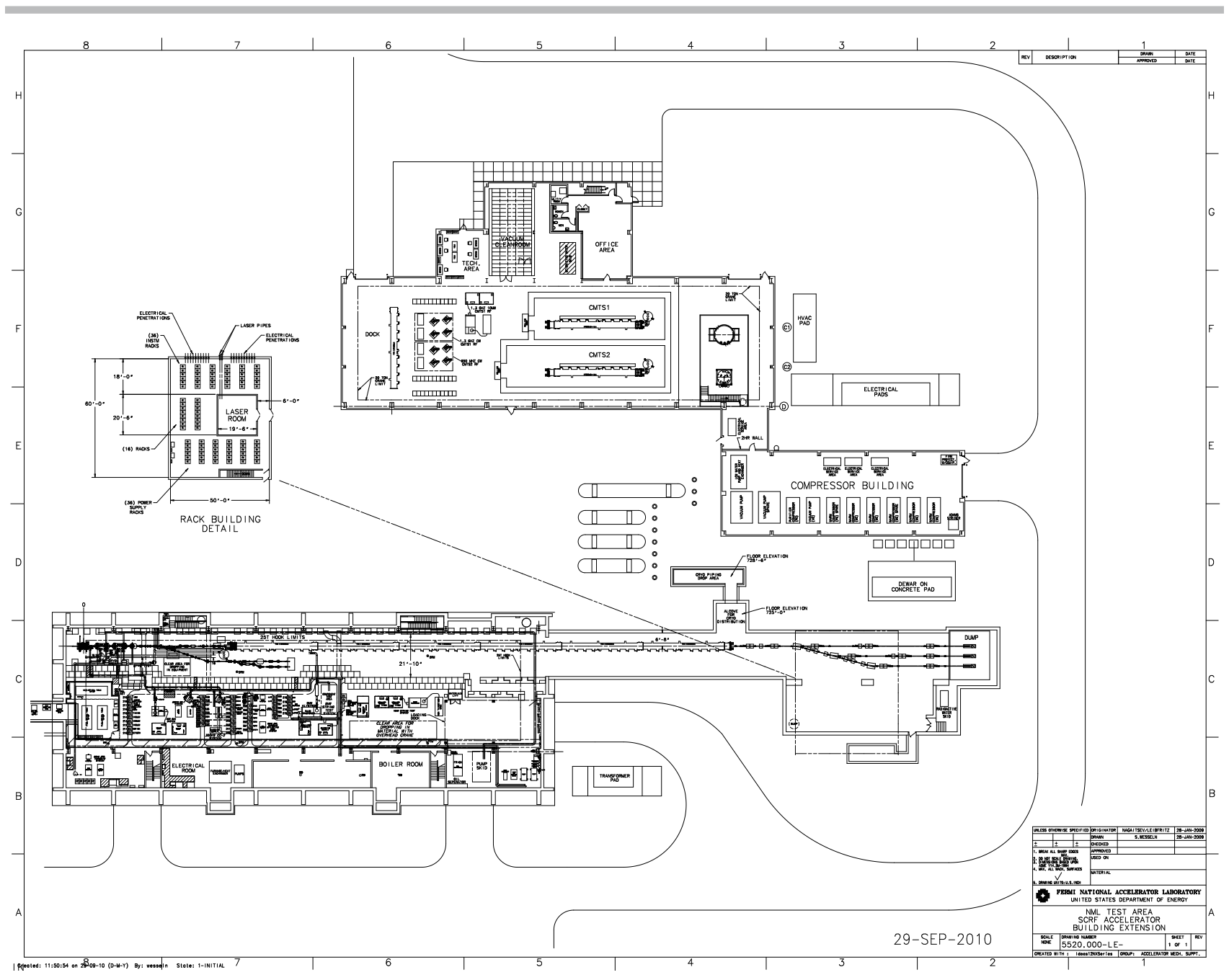

Figure 2.11 General layout of NML at Fermilab. 


\subsubsection{Development of infrastructure in Europe}

The European SCRF activities are naturally centred on the imminent mass production for the European XFEL. The contracts for the production of some 600 cavities, couplers and tuners have been extended to industry. This development marks an important milestone since the cavity treatment process had to be agreed upon in all detail and specified for mass production. It is also an important milestone for the ILC since, although a higher operating gradient is required for the ILC, the manufacture with final electropolishing is deemed identical for European XFEL and ILC. Cryomodules have been produced by various vendors and performed excellently at the FLASH facility so that a sufficient industrial base has become available. Initial cold tests of the fabricated cavities will be made at DESY. RF couplers procured, tested and conditioned by CNRS/LAL (in Orsay, France) will be mounted at CEA/Irfu (in Saclay, France), who will also carry out the string and overall accelerator module assembly [2-24]. Subsequently the cryomodules will be returned to DESY for test and installation into the European XFEL.

The manufacturing contracts include 24 cavities that will be available for additional treatment for highest gradients and supplemented with couplers and tuners. These cavities are part of the ILC-HiGrade project that addresses the high gradient in the context of cavity mass production.

Surface preparation (polishing) of the cavities requires first a bulk removal process, followed by a final 'fine polishing'. The ILC specification requires that both bulk and final treatments be made using electropolishing. For the XFEL, electropolishing is used for the bulk treatment of all the cavities, but the final polishing process will be either electropolishing or buffered chemical polishing, depending on the vendor. The vendors are required to meticulously follow the process description and to document the individual steps. A comparison of the two techniques for final surface treatment can be done based on the statistics of several hundred cavities each.


Figure 2.12 Left: tool for room temperature RF measurement of half cells. Right: tool for field flatness tuning of completed cavities. 
Figure 2.13 Layout of the Accelerator Module Test Facility hall at DESY.
The two manufacturers selected in the tendering process will be supplied with European standards certified tools developed by DESY and Fermilab to assess the field and mechanically tune the individual cells for field flatness. These automated tools can be operated by non-RF experts and will provide a standardised log of the recorded data at the same time. The automation has considerably reduced the tuning time. Figure 2.12 shows the automated RF measurement and the tuning machines.

The first cold test of the nine-cell cavity performance will be made at DESY. It will provide the first feedback on the achieved gradient after manufacture. All cavities, except for the ILC-HiGrade cavities, will be delivered with the helium vessel mounted. Much of the existing infrastructure at DESY has been developed at the time of the TESLA Technology Collaboration and has been used ever since. Evidently this infrastructure had to be augmented to allow for bulk testing. The Accelerator Module Test Facility has recently been built to allow for series test of cavities delivered from the manufacturer. Figure 2.13 shows the general layout.

Here four cavities at a time will be mounted on the so-called insert as shown in Figure 2.14. It is subsequently lowered into the vertical cryostat and individually tested for performance, which includes a test of peak field performance.

The facility has been planned for minimal physical handling of the cavities to guarantee high throughput and reproducibility. The test procedure was standardised and aims for rapid characterisation of the cavity performance. It is expected that most of the cavities will pass this test and fulfil the gradient requirement for the European XFEL of $22.4 \mathrm{MV} / \mathrm{m}$. We estimate based on current understanding that the treatment of cavities of higher gradient will be no different. Cavities that fail the acceptance test will have to be diagnosed in detail and are subject to post-processing. The reason for failure is typically field emission from the inclusion of particulates in the niobium surface, or a quench resulting from surface irregularities. The vertical cryostats will be equipped with sensors, called oscillating superleak transducers, for detection of second-sound signals originating from the source of the cavity quench. The analysis of the second-sound propagation time enables the reconstruction of the quench origin by triangulation. 


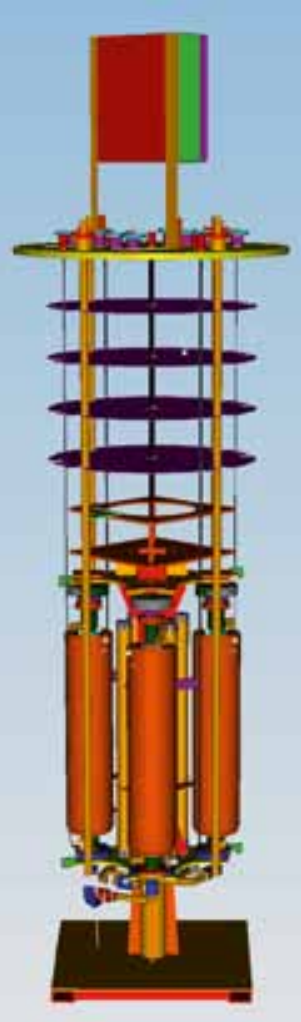

Figure 2.14 The cavity insert holds four cavities at a time for the test in the vertical cryostat.

ILC-HiGrade is also preparing an automated optical scanner, which will map the surface close to the electron beam weld of cell hemispheres and the iris between two cells. The optical scanner uses the Kyoto-KEK developed camera, as described above, which resolves surface features down to about $10 \mu \mathrm{m}$.

The long existing infrastructure at DESY has also been improved in order to facilitate the post-processing. The cleanroom standards have been raised and the handling steps have been streamlined. The failing cavities lend themselves to an additional short buffered chemical polish process, which removes a layer of niobium of around $10 \mu \mathrm{m}$ from the surface. Specific action can be taken to address surface features located in the optical scan.

The accepted cavities will be shipped to CEA/Irfu for further assembly. The actual shipping procedure has been verified; the shipping tools have been developed and the forces on the cavities during transport have been measured and found acceptable.

The high-power couplers will be manufactured in industry and are delivered to CNRS/LAL for conditioning. Eight couplers will be conditioned per week with a maximum RF power of 5 megawatts (MW). Figure 2.15 shows the layout of the coupler conditioning facility at LAL. After acceptance test the couplers will be shipped directly to the assembly site at CEA/Irfu. 
Figure 2.15 Layout of the coupler conditioning facility at CNRS/LAL.
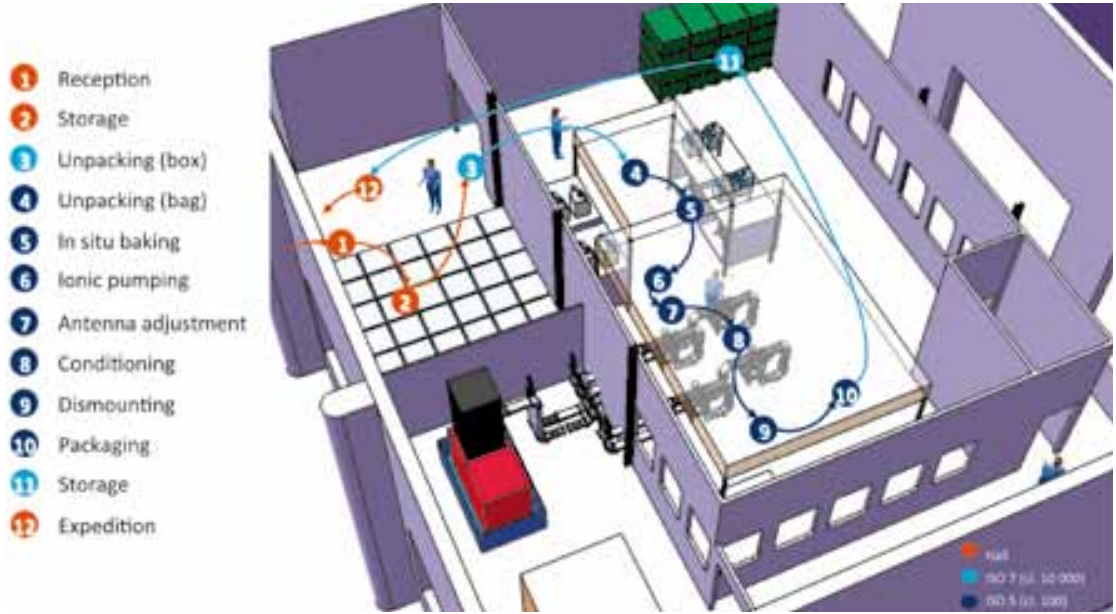

Figure 2.16 Layout of the cryomodule assembly facility at CEA/Irfu.
The team at CEA/Irfu has equipped existing lab space to house the infrastructure for cryomodule assembly [2-24]. Figure 2.16 shows the general layout. All components will be 'washed' before assembly. A large ISO 4 cleanroom and supplemental ISO 5 and ISO 7 cleanrooms have been installed to allow for mounting of the supplied couplers and for assembly of the cavities into a string. Once assembled, the string will be precision-aligned. The cantilever system will be used to insert the string into the cryomodule. The infrastructure will initially be used for the components for the SPIRAL2 project, and then for mass production for the European XFEL. 


\subsubsection{Overview}

The gradient choice for the ILC SCRF cavities is important for the beam energy reach and the machine cost. At the time of the RDR, a choice of $35 \mathrm{MV} / \mathrm{m}$ was made for cavity vertical qualification tests [2-25]. This choice was supported by the demonstration of a gradient of $35 \mathrm{MV} / \mathrm{m}$ or more in several nine-cell TESLA Test Facility (TTF)-shape cavities, results from the DESY-KEK collaboration. These cavities were surface-processed by electropolishing, heat treated at $600-800{ }^{\circ} \mathrm{C}$ in a vacuum furnace for hydrogen removal and baked at $120^{\circ} \mathrm{C}$ for 48 hours after the final EP.

Achieving $35 \mathrm{MV} / \mathrm{m}$ in nine-cell cavities reproducibly is important, as the total number of cavities required for the ILC is far more than any SCRFbased machines built or planned. A global R\&D programme called So was established in 2006 to address this challenge [2-26]. The So programme, coordinated by the GDE Cavity Group, has broad global participation from ANL, Cornell, DESY, Fermilab, JLab, and KEK. IHEP and Peking University are also going to participate in the programme. Significant progress in understanding the gradient limit and gradient scatter has been made by instrumented cavity testing at cryogenic temperatures and high-resolution optical inspection of the cavity RF surface. This is accompanied by steady progress in reproducibility at $35 \mathrm{MV} / \mathrm{m}$ and in improved practical gradient limit in nine-cell cavities. At the time of the RDR, Europe was the only region to have demonstrated $35-\mathrm{MV} / \mathrm{m}$ nine-cell cavity fabrication and processing. Today, 35-MV/m cavity fabrication and processing has also been demonstrated in the Asia and Americas regions. A solid SCRF technical base for the ILC on a global scale is now in place.

The global efforts in ILC gradient R\&D are rewarded not only by improved gradient yield and reproducibility but also in the achievement of still higher gradients. By mid-2010, a major SCRF gradient R\&D milestone of 50\% yield at $35 \mathrm{MV} / \mathrm{m}$ was achieved as described below. The average gradient in the stateof-the-art nine-cell cavities is raised to around $40 \mathrm{MV} / \mathrm{m}$, a steady increase compared to the state-of-the-art $35 \mathrm{MV} / \mathrm{m}$ in 2005.

\subsection{PROGRESS TOWARDS MANUFACTURE OF HIGH- GRADIENT CAVITIES}

\footnotetext{
2.3.2 Globally coordinated gradient R\&D - the S0 programme

The ILC gradient R\&D is a global effort with current participation of ANL, Cornell, DESY, Fermilab, JLab, and KEK. Information is exchanged monthly at the GDE ILC Cavity Group meetings. Diagnostic tools, production procedures and process parameters are at times verified by exchanging cavities across the labs and regions. There is growing interest and capability in cavity gradient R\&D in other labs such as IHEP and Peking University in China, TRIUMF in Canada, and RRCAT and IUAC in India. Encouraging cavity results are emerging. Historically, these R\&D initiatives usually drive industrial interest and capability for SCRF cavity manufacture and processing. A global SCRF industry is emerging, driven in large part by the demand for higher cavity gradient for the ILC.
} 


\subsubsection{Understanding the source of gradient limitation and scatter}

The initial So effort focused on the issue of field emission, which was identified to be the main cause of gradient variability. Following the recommendation of the TESLA Technology Collaboration 2005 report, R\&D priority was given to improved post-EP cleaning procedures [2-27]. Three methods are now established for effective field emission reduction: ethanol rinsing was successfully developed and applied at DESY, ultrasonic cleaning with detergent was introduced and optimised at JLab and fresh EP was found effective for field emission reduction at KEK. Some of the methods have been successfully transferred across facilities at different labs.

As a result of the R\&D effort, sources of field emission are now understood [2-27]. Besides the traditional particulate field emitters, niobium oxide granule is found to be a major field emitter introduced by the electropolishing process itself. Wiping and brushing of end group components immediately after EP processing reduces niobium oxide granules (often accompanied by increased sulphur-bearing compounds) in the hidden areas where high-pressure rinse cleaning is less effective due to lack of direct water jet bombardment. Streamlined cleanroom assembly procedures are now routinely used, minimising recontamination by particulates generated by the assembly process itself. 'Field emissionfree' performance up to $40 \mathrm{MV} / \mathrm{m}$ has been demonstrated in several electropolished nine-cell cavities. Efforts are continuing to develop improved and new cleaning techniques towards the goal of eradicating field emission up to the theoretical quench limit of a niobium cavity.

Our understanding of the source of limiting quench behaviour is much improved thanks to temperature mapping measurements and accompanying high-resolution optical inspection. Many of the thermometry and inspection instruments have been successfully adopted in most labs participating in the So studies. Kyoto-KEK cameras and Cornell oscillating superleak transducer systems are two examples. For the ILC nine-cell cavities, a quench limit at below $25 \mathrm{MV} / \mathrm{m}$ is found to be caused predominantly by highly localised geometrical defects in the zone adjacent to the electron beam weld seam at the cavity equator. These defects can be roughly categorised as circular pits or bumps with a typical diameter in the range of 200 to $800 \mathrm{~mm}$. Detailed morphology of the defect can be quite complicated as revealed by replica mould measurements and by microscopic inspection of small samples cut out from real cavities. It is suspected that local magnetic field enhancement at sharp edges are usually observable on the RF surface of as-built cavities. This quench limit due to geometrical defects is insensitive to repeated EP, and there is emerging evidence to show that these geometrical defects already exist before any chemistry is done to the cavity surface. It is now generally recognised that, in order to overcome quench limits below $25 \mathrm{MV} / \mathrm{m}$, improved quality assurance and control in material and fabrication are important. 
Further studies are needed to understand the source of quenches at higher gradients in the nominal range of above $30 \mathrm{MV} / \mathrm{m}$. It is clear that higher quench limits are also caused by highly localised defects near the equator electron beam weld, but unlike the geometrical defects, there is typically no observable feature at the predicted quench site. It has been suggested that locally suppressed superconductivity due to compositional irregularities may be responsible. Additional capabilities of compositional analysis in situ at the predicted quench location are expected to shed light on this issue, and offline microscopic analysis of small samples removed from the predicted quench location (as has been done at DESY) may also provide important information for further gradient improvement.

\subsubsection{Gradient yield definition and global cavity result database}

At the start of the Technical Design Phase, goals of a $50 \%$ process yield by 2010 and a 90\% production yield at $35 \mathrm{MV} / \mathrm{m}$, with a quality factor $\mathrm{Q}_{0}$ of greater than $8 \times 10^{9}$, were defined. As the TDP progressed, the needs for a clearer definition of the production yield and for a globally consistent and available database for recording test results were recognised. In 2009 the GDE ILC Cavity Group proposed a clear definition of gradient yield, which adopted the concept of the first-pass and second-pass yield and established rules for cavity selection. In parallel the ILC Global Cavity Database Team was created as a part of the So effort [2-28]. The team includes members from Cornell, DESY, Fermilab, JLab and KEK, and took on the task of not only creating the database, but also defining the rules for how the data should be included and how the data would be presented. The result of this effort is a clear, objective, and publicly accessible database where the progress of cavity $\mathrm{R} \& \mathrm{D}$ can be tracked. In fact, the database group has presented status reports at each major ILC workshop, meeting or review since mid-20o9.

The most recent results for first-pass and second-pass yields, presented in a time-phased manner at the IWLC2010 meeting in Geneva, Switzerland, are shown in Figure 2.17 [2-29]. By way of definition, plots only include results from a vendor-laboratory combination that has previously demonstrated through tests the ability to fabricate and process a cavity that achieves more than $35 \mathrm{MV} / \mathrm{m}$ in a vertical test. A first-pass result is one where the fabrication and processing have been completed according to the standard recipe leading up to the first test; a second-pass result sums up all first pass results greater than $35 \mathrm{MV} / \mathrm{m}$ and those results where a poorer-performing cavity had some remediation applied based on diagnostics from the first test. Apparent from the graphs is the improvement with time of the yield curves, particularly for second-pass results. This improvement is attributable to improved diagnostic and remediation tools that have been developed in the past years. Repeatability in EP processing, one of the methods that has been demonstrated, plays an important role in improving the second pass yield by raising the gradient performance of cavities quench limited between 25 and $35 \mathrm{MV} / \mathrm{m}$. A smaller gain is seen in the first-pass results. This is consistent with our limited ability to recognise fabrication flaws early in fabrication by means other than vertical testing. Improvement of our understanding of the critical fabrication parameters and the development of predictive quality assurance checks are an R\&D direction in the remainder of the TD phase. 
Figure 2.17 Cavity gradient performance with production yield for (top) the first pass and (bottom) the second pass.
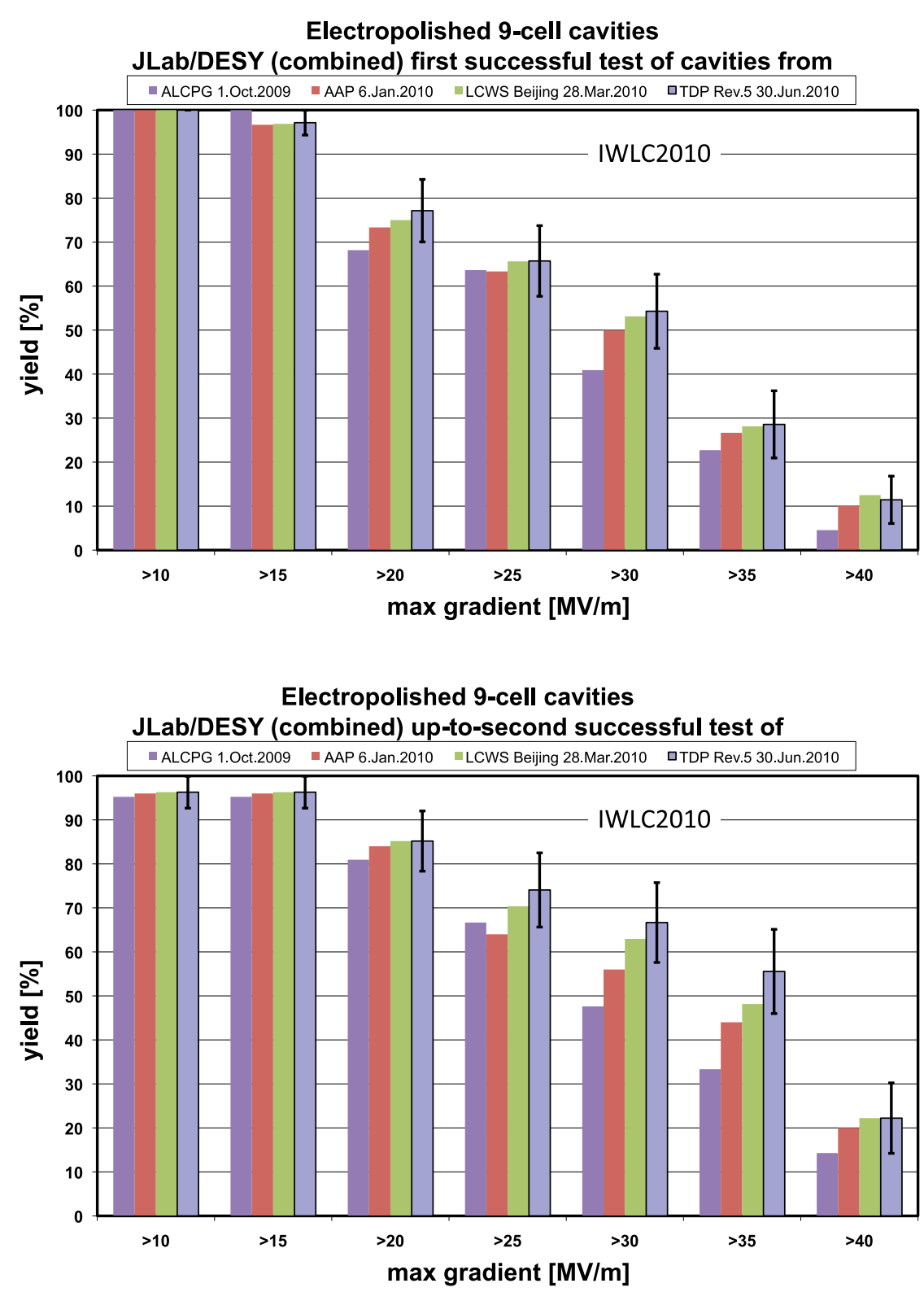

2.3.5 Achieving the TDP-1 gradient milestone of $50 \%$ yield at $35 \mathrm{MV} / \mathrm{m}$ The improved understanding of gradient limit goes hand in hand with the improvements achieved in cavity gradient yield. Field emission was much reduced due to the application of post-EP cleaning procedures such as ethanol rinsing and ultrasonic cleaning with detergent, with a further reduction in field emission achieved by applying the procedure of continued acid circulation after the EP voltage is turned off. This procedure reduces sulphur-bearing niobium oxide granules and hence reduces inherent contaminants on the as-polished surface. Optimised electropolishing and streamlined cleanroom assembly resulted in reproducible cavity processing and hence reproducible cavity gradient results. As a result of the continued 
improvement and optimisation of the cavity processing and the continued understanding of the gradient limit, the TDP-1 gradient goal of a $50 \%$

production yield at $35 \mathrm{MV} / \mathrm{m}$ and at $Q_{\mathrm{o}}$ greater than $8 \times 10^{9}$ was accomplished in June 2010 as shown in Figure 2.17. Further highlighted individual institutional progress in the gradient R\&D in 2010 is summarised in Table 2.4.

\begin{tabular}{|c|c|c|}
\hline Joint effort & Progress & Table 2.4 Globally highlighted gradient R\&D \\
\hline Research Instruments-JLab & Achieved $90 \%$ yield at $\geq 35 \mathrm{MV} / \mathrm{m}$ and $\mathrm{Q}_{0} \geq 8 \times 10^{9}$ & progress in 2010. \\
\hline $\begin{array}{l}\text { Research Instruments- } \\
\text { Fermilab/ANL/JLab }\end{array}$ & Achieved $\geq 35 \mathrm{MV} / \mathrm{m}$ and $\mathrm{Q}_{0} \geq 8 \times 10^{9}$ & \\
\hline $\begin{array}{l}\text { Research Instruments- } \\
\text { Fermilab/ANL }\end{array}$ & Achieved $34.5 \mathrm{MV} / \mathrm{m}$ with tumbled cavity & \\
\hline Niowave-Fermilab/ANL & Achieved $28.8 \mathrm{MV} / \mathrm{m}$ with the first production cavity & \\
\hline KEK-Fermilab/ANL & Demonstrated local repairing: gradient improved from 11 to $30 \mathrm{MV} / \mathrm{m}$ & \\
\hline IHEP-KEK & Achieved $20 \mathrm{MV} / \mathrm{m}$ with the first IHEP cavity (LL, LG, no-end) & \\
\hline PKU-JLab & Achieved $28 \mathrm{MV} / \mathrm{m}$ with the PKU cavity (TESLA, FG, w/-end) & \\
\hline Hitachi-KEK & Achieved $35 \mathrm{MV} / \mathrm{m}$ with the first Hitachi cavity (TESLA-like, FG, no-end) & \\
\hline MHI-KEK & Achieved $\geq 35 \mathrm{MV} / \mathrm{m}$ and $\mathrm{Q}_{0} \geq 8 \times 10^{9}$ with MHI- 12 cavity & \\
\hline DESY/E-XFEL & 600 cavities ordered; RI and Zanon awarded & \\
\hline
\end{tabular}

\subsubsection{Aiming at the TDP-2 gradient goal and $90 \%$ yield at $35 \mathrm{MV} / \mathrm{m}$}

After successful achievement of the 2010 goal of $50 \%$ second-pass production yield, the TDP-2 2012 goal remains a cavity gradient second-pass production yield of 90\%. This is an ambitious goal, but appears possible. Recently, a 90\% yield has been demonstrated based on very limited statistics (ten cavities built by one of the most experienced cavity manufacturers and processed and tested at JLab). Our efforts in TDP-2 will focus on two areas: at lower gradients, the modification of the production process to remove mechanical pits and other imperfections that now appear to be a leading cause of lower-gradient quench limitations; and at higher gradients, improvement of the processing and assembly techniques that result in improved surface homogeneity and reduced field emission. Though both of these efforts will start in our cavity R\&D efforts with remediation of defects or emission seen in the first-pass results, the goal is to understand the problems well enough that our efforts can become predictive, rather than reactive. Our ultimate goal is to feed knowledge from labs back to industry for improved fabrication in industrial manufacturers.

For mechanical defects, use of inspection systems such as the Kyoto-KEK camera, silicone pit modelling or moulding, or X-ray tomography to locate and categorise defects early in production, and tracing of these defects to performance-limiting locations as seen by T-mapping or second sound, will require added inspection efforts over the next years to create a database of defects and a more detailed understanding of the parameters that directly limit performance. For processing errors, more detailed understanding 
of the process itself is required, as is reasonable quality assurance checks to make sure the processes are executed successfully each time. This will include further tweaking of the standard process formula.

Finally, it should be noted that neither of the above R\&D directions has been proven to be conclusively 'the' answer to the current yield limits. Current performance limitation could vary from vendor to vendor or from laboratory to laboratory. Continued incremental improvements will rely on continued extensive inspections, until the exact root causes are proven.

\subsubsection{Long-term cavity R\&D for a very high gradient beyond the TDR} Long-term R\&D addresses the gradient need for the ILC 1-TeV upgrade [2-27]. With the improved cavity cell shapes and optimised material properties, one can expect nine-cell niobium cavities with gradients in the range of 40 to $60 \mathrm{MV} / \mathrm{m}$. There are three proposed cell shapes with major efforts for very high gradients. The low-loss shape developed at KEK has shown excellent gradient results of around $50 \mathrm{MV} / \mathrm{m}$ in many single-cell cavities [2-30]. Several nine-cell cavities have been prototyped and gradients of more than $35 \mathrm{MV} / \mathrm{m}$ have been demonstrated in nine-cell cavities with and without end-groups. The re-entrant shape developed at Cornell has shown the record gradient of around $60 \mathrm{MV} / \mathrm{m}$ in a single-cell cavity [2-31]. The first nine-cell re-entrant cavity has been built and efforts are underway to push for very high gradient. A new 'low surface field' shape has been designed at SLAC. Planning is underway at JLab to prototype the first single-cell and multi-cell low surface field shape cavities.

In addition to the cell shape development, one can also expect benefits from continued optimisation of niobium material. There is evidence that heat treatment, at various stages such as post-forming or post-fabrication, may have significant room for improvement in achieving very high gradients. As the shape improvement and material improvement are two independent paths and as both are compatible with the EP processing procedure, one can expect significant gradient improvement toward the range of 40 to $60 \mathrm{MV} / \mathrm{m}$ by using the current baseline cavity processing procedures.

It is generally agreed that gradients up to $100 \mathrm{MV} / \mathrm{m}$ in superconducting RF cavities are theoretically possible by switching to other superconducting materials, but significant fundamental $R \& D$ is required. Active programmes now exist in this direction pursued by several groups at ANL, Cornell and JLab. It is recognised that the most promising path lies in the thin film coating of new material on copper or aluminium substrates pre-formed into suitable cavity shapes. Given the potential high return, R\&D of new superconducting RF material and cavity system development should be intensified after the TDR in order to support the physics scenario of $1 \mathrm{TeV}$ or more for the ILC. 
Most of the effort and resources spent on ILC cryomodule activities since the publication of the RDR and the beginning of the Technical Design

Phase have been devoted to the collaborative S1-Global programme [2-32]. Taking advantage of experimental activities at KEK in the framework of this worldwide effort, several ILC cryomodule design issues have been addressed, like the assessment of the thermal performance of the intermediate 5-kelvin (K) shields and the investigation of diverse tuner and magnetic shielding solutions. The experience gained from S1-Global, which integrates different variations of components (e.g., cavities, magnetic shielding, couplers and tuners) from several collaborating partners into a single cryomodule, is also very important for the assessment of all the 'plug-compatible' interfaces of the cryomodule for future ILC plans.

\subsection{CRYOMODULE DESIGN AND DEVELOPMENT}

\subsubsection{Progress in the S1-Global cryomodule development hosted at KEK}

The ILC cryomodule design study mainly done at KEK is only briefly described here. (See 2.6.2 for details of the high-powered RF tests.) The cryomodule design work began in May 2008 with a joint team of Fermilab, INFN and KEK, and was completed by the end of 2008. For construction of the S1-Global cryomodule, KEK performed the modification of a half cryomodule, so-called Cryomodule-A, from one of the original STF modules [2-33] in 2009, while INFN and KEK cooperatively provided components for another half cryomodule, so-called Cryomodule-C. DESY, Fermilab and KEK provided and tested eight cavities and couplers needed for the S1-Global programme. The S1-Global cryomodule was assembled and installed in the KEK-STF tunnel between January and May 2010. The cold tests were performed between June 2010 and February 2011. Before starting the S1-Global cryomodule cold test, KEK completed the thermal test of the intermediate 5 -K shield by using a half-length, 6-m-long STF cryomodule. The heat load to the $2-\mathrm{K}$ region was measured with and without the $5-\mathrm{K}$ shield and compared with calculations. The results are being applied on the thermal design of the ILC cryomodule. The thermal design guideline for the ILC cryomodule will be summarised below.

\subsubsection{Progress in the European XFEL project hosted at DESY and R\&D at Fermilab}

The European XFEL project is now in its construction phase and the commissioning is expected in 2014 [2-34]. The XFEL superconducting linac consists of 80 cryomodules with a design derived from the TESLA Test Facility Type-3 on which the ILC module design concept is also based. The industrial vendors have been qualified, and the project is moving forward to the start of the serial production. The European XFEL project will therefore soon provide much experience from a relatively large series of production modules in an industrial context and that will bring valuable information for the industrialisation of the ILC cryomodules. 
At Fermilab, CM-1, an ILC Type-3 cryomodule assembled using parts provided by DESY, INFN and Fermilab, has been cooled down and tests are underway at the NML test facility at Fermilab. Dressed cavities for CM-2 are being tested in Fermilab's Horizontal Test Cryostat. In addition, design work is in progress on cryomodules for Project $\mathrm{X}$, a high-intensity proton linac proposed for Fermilab's next generation of neutrino experiments and other studies. Project X cryomodules, being a continuous wave and low beta design, differ in various ways from ILC-type cryomodules but will benefit greatly from the ILC experience.

\subsubsection{ILC cryomodule design with plug compatibility}

In order to develop an industrialised design of the ILC cryomodule, plugcompatible interfaces of the cryomodule have been defined as listed in Table 2.5. A detailed and more complete parameter list shall be established in future studies.

Table 2.5 Plug-compatible interfaces of cryomodule.

Table 2. 6 Heat loads of one

ILC cryomodule from RDR.

\begin{tabular}{l} 
Interface \\
\hline Cryomodule slot \\
\hline Vacuum vessel \\
\hline Vacuum bellow \\
\hline Vacuum flange
\end{tabular}

Item

Length

Length/Outer diameter/Support

Length

Input coupler interface

to vacuum vessel

Cavity support lug

Cooling pipes

Diameter/Thickness/Connection

Diameter/Thickness/Connection

Span/Lug width and thickness

Position/Diameter/Material

Parameter

$12,679.6 \mathrm{~mm}$

$11,830 \mathrm{~mm} / 965.2 \mathrm{~mm} / \mathrm{TBD}$

$849.6 \mathrm{~mm}$

TBD

Longitudinal pitch: $12,679.6 \mathrm{~mm}$ Others: TBD

$750 \mathrm{~mm} / \mathrm{TBD}$

Cooling pipe parameters are specified w/ cryogenic design.

\subsubsection{Study of thermal balance with and without 5-K radiation shield}

The specific heat loads in the three cryogenic circuits of one ILC cryomodule (at the temperature levels of $2 \mathrm{~K}, 5 \mathrm{~K}$ and $40 \mathrm{~K}$ ) are listed in Table 2.6, calculated from the values of one RF unit as shown in the RDR [2-35].

\begin{tabular}{|c|c|}
\hline Load, in W & $2 \mathrm{~K}$ \\
\hline Static load & 1.7 \\
\hline Dynamic load & 9.7 \\
\hline Total load & 11.4 \\
\hline
\end{tabular}

\begin{tabular}{l}
$5 \mathbf{K}$ \\
\hline 10.6 \\
\hline 4.4 \\
\hline 15.0
\end{tabular}

\begin{tabular}{l}
$40 \mathrm{~K}$ \\
\hline 59.2 \\
\hline 94.3 \\
\hline 153.5
\end{tabular}


In the 5 - $\mathrm{K}$ circuit, the heat load by thermal radiation was evaluated to be at $1.41 \mathrm{~W}$. The present ILC cryomodule scheme, derived from the TESLA Test Facility cryomodules [2-36], has two thermal shields circuits. The ' 5 -K circuit' contains helium pressurised above the 2.3-bar critical pressure, which warms from $5 \mathrm{~K}$ to $8 \mathrm{~K}$ through the circuit.

The $40-\mathrm{K}$ circuit contains helium gas warming from $40 \mathrm{~K}$ to $80 \mathrm{~K}$. When the $5-\mathrm{K}$ thermal radiation shield is removed (this was investigated as a means to simplify the module and decrease the fabrication costs), thermal radiation from the $40-\mathrm{K}$ screen would directly impinge into the $2-\mathrm{K}$ region, increasing noticeably the module's $2-\mathrm{K}$ heat load. However, by modifying the $40-\mathrm{K}$ thermal shield flow scheme as shown in Figure 2.18 [2-37] and by rearranging the thermal intercept strategy, this additional contribution could be mitigated. The improvement is obtained by using the forward line to lower the temperature of the thermal shield from $74 \mathrm{~K}$ to $46 \mathrm{~K}$ while using the return (warmer) line to provide the thermal intercepts of input couplers and current leads, thus increasing the average intercept temperature from $54 \mathrm{~K}$ to $66 \mathrm{~K}$.

\section{Allocation of thermal loads to $40 \mathrm{~K}-80 \mathrm{~K}$ circuit}

$300 \mathrm{~K}$ wall of cryostat

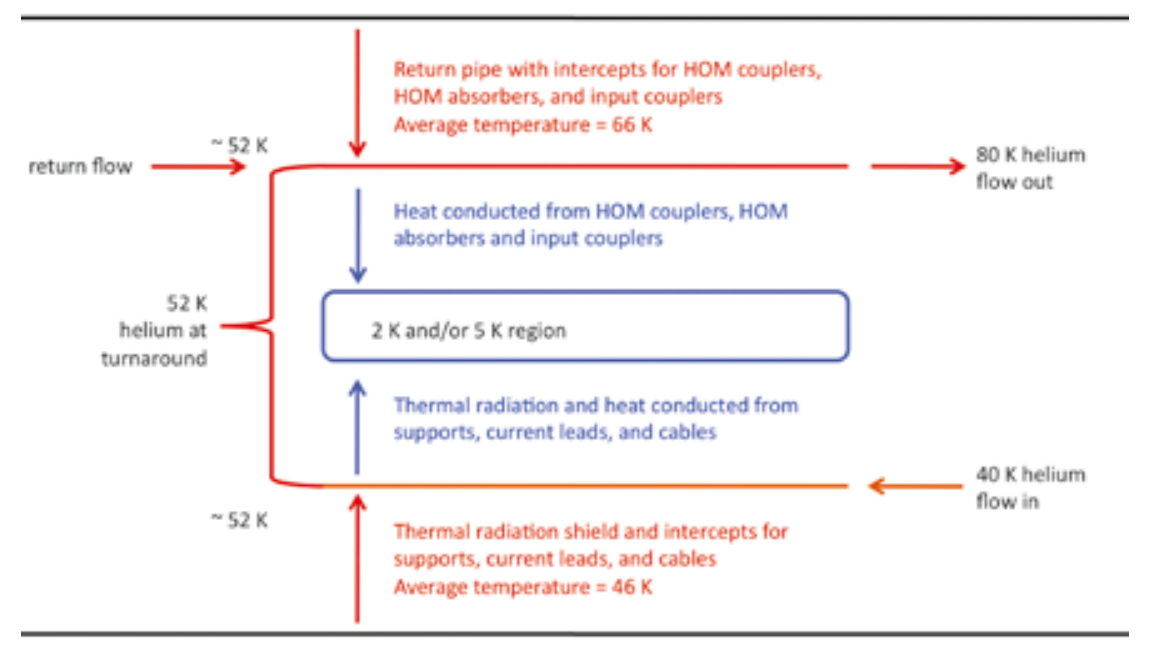

$300 \mathrm{~K}$ wall of cryostat
Figure 2.18 Cooling scheme and thermal balance for the ILC cryomodule's thermal design.

For future assessment of the ILC cryomodule's thermal design, the cost implications of eliminating the lower part of the $5-\mathrm{K}$ shield needs to be carefully studied, particularly in the following aspects:

1. Capital and assembly cost reduction of the lower shield components

2. Capital and operational cost increase of the cryogenic system due to the additional heat load 
Figure 2.19 Cross-section of the cryomodule with (left) two thermal shields and (right) without the lower part of 5-K shield.
The effect on the assembly time and costs for providing the 5 - $\mathrm{K}$ thermal intercept to other components, such as main and HOM couplers, will be studied. For example, the cross-sections of the cryomodule with the 5-K shield and without the lower part of the 5-K shield are shown in Figure 2.19. In the proposed design, the cooling line at $5 \mathrm{~K}$ is preserved due to the need to provide the thermal intercepts of the input couplers, support posts and RF cables.

\subsubsection{Study of the magnetic shield assembly}

Different magnetic shield solutions are being developed by several institutions and are being studied from a viewpoint of the cryomodule assembly work. The magnetic shields of DESY and Fermilab (TESLA-type) cavities are designed to be assembled outside of the cavity jacket and require more numbers of split components. The magnetic shield of the KEK (TESLA-like) cavity is designed to be internally installed in the helium jacket and requires fewer split components. Both designs have been verified to work well through the S1-Global cavity string test. Further detailed design studies need to be carried out to find the best cost-effective magnetic shield design as a part of the best cost effective cryomodule design, satisfying the magnetic shield performance. 
In the Reference Design Report (RDR) [2-38], the high-power radiofrequency equipment and layout had reached a fairly mature state. The klystrons, modulators and related equipment were housed in a parallel utility tunnel, connected every $38 \mathrm{~m}$ to the main linac tunnel by a penetration through which waveguides carried up to $10 \mathrm{MW}$ of Lband $(1.3 \mathrm{GHz})$ power, to be distributed along three cryomodules. Extensive ongoing work has been required, due mainly to the fundamental change in tunnel configuration. With the move to single-tunnel main linac housings, the production and distribution to the cavities of RF power had to be rethought. Two proposed options are currently being pursued by ILC R\&D programmes. They are referred to by the acronyms KCS (klystron cluster scheme) and DRFS (distributed RF system).

\subsection{HIGH-POWER RADIOFREQUENCY DEVELOPMENT}

\subsubsection{Klystron cluster scheme}

\section{System description}

In the KCS scheme [2-39], RF production is moved to the surface. Unlike in the $\mathrm{RDR}$, where it is brought down as alternating current $(\mathrm{AC})$ wall-plug power, or the European XFEL, where it is brought down as direct current (DC) cable power to underground klystrons, with KCS, the power used to accelerate the beam is transported between the surface and the underground tunnel as RF. This approach of sending power down as RF follows the example of the SLAC linac, which served the only previous linear collider, the SLC. The differences arise both from having to accommodate a deep-bored (as opposed to cut-andcover) tunnel, which makes shafts expensive, and from the need to minimise surface impact over what will be a much larger footprint.

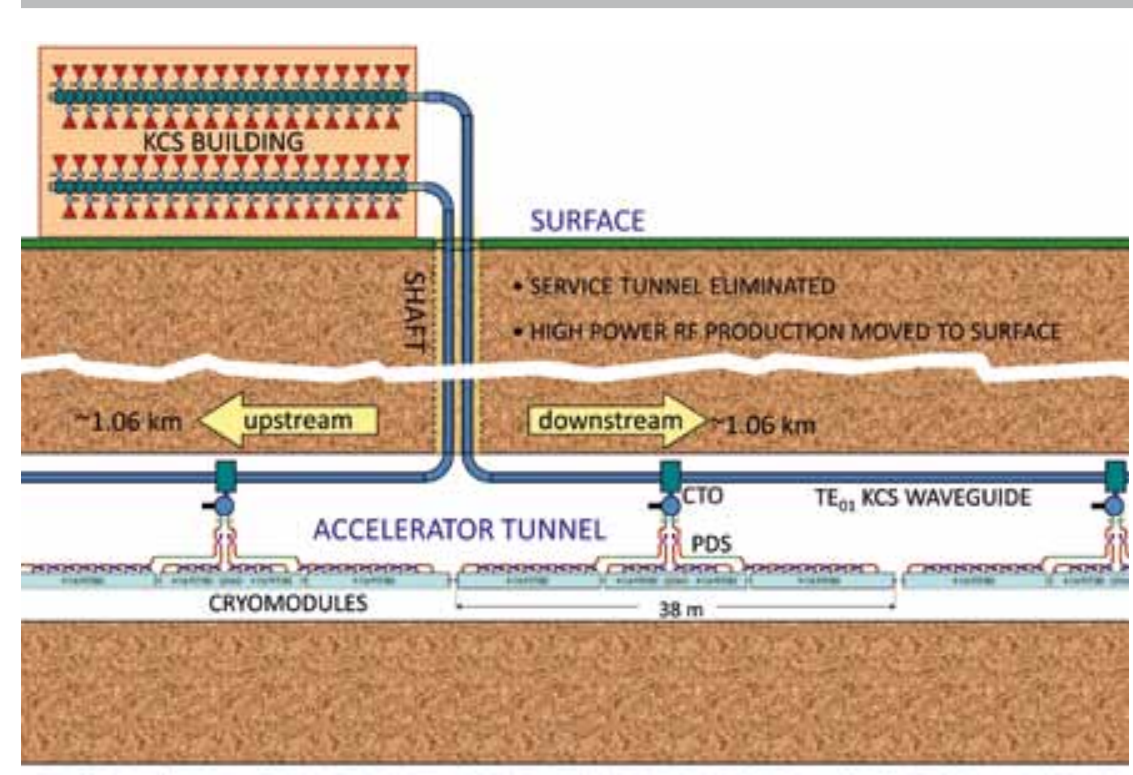

Figure 2.20 Basic layout scheme of the klystron cluster scheme. Many high-power RF sources in a surface building are combined into a large circular waveguide. 
Thus the idea of clustering was adopted. Power from groups of approximately 34 10-MW klystrons is combined into a single low-loss, over-moded waveguide and transported down to the tunnel and along the linac as shown in Figure 2.20. This is sufficient, allowing for a few percent extra transmission loss, to power approximately $1.06 \mathrm{~km}$ of ILC linac. At $38-\mathrm{m}$ intervals, partial power is siphoned from this main waveguide in 10-MW decrements. From each such tap-off, the RF is distributed to the cavities (26 in three cryomodules) through a WR650 waveguide system, just as it would be from a local klystron.

To minimise the number of buildings and shafts required, each houses two klystron clusters with one feeding upstream and the other downstream, powering over $2 \mathrm{~km}$ of linac per shaft. Besides the KCS main waveguide artery, some equipment from the service tunnel, such as front-end feedback electronics and beam instrumentation, is added to the linac tunnel in shielded crates below the cryomodules. No increase in tunnel diameter is foreseen. In addition to the civil construction cost savings associated with going to a single tunnel, this scheme brings the heat load associated with the RF production equipment to the surface, greatly facilitating cooling.

\section{Klystrons and modulators}

In the RDR, a 10-MW multi-beam klystron was identified as the ILC L-band power source. This choice was based on cost effectiveness, efficiency and relatively low operating voltage, and takes advantage of years of R\&D for TELSA and the European XFEL. The prototypes were built by CPI, Thales, and Toshiba. The KCS option retains this source. A Toshiba tube is being operated at SLAC for reliability and lifetime studies.

The modulator requirements for the klystrons are 120-kilovolt, 140-A, 1.6-millisecond pulses at a $5-\mathrm{Hertz}(\mathrm{Hz})$ repetition rate. As an alternative to the RDR baseline design, SLAC is pursuing a Marx-topology modulator to fulfil this requirement with a reliable and cost-effective approach [2-40]. A fullscale prototype, the SLAC P1 Marx is currently undergoing lifetime testing, driving the above-mentioned 10-MW Toshiba multi-beam klystron. In the accumulated 1,500 hours there have been no chronic problems. A secondgeneration Marx, the SLAC P2 Marx, is currently under development [2-41].

The Marx is made up of many identical and, ideally, redundant cells. If a cell becomes inoperable, it can be bypassed. Increasing the applied charge voltage or turning on 'spare' cells allows the modulator to continue operation. In addition, a modular design allows better use of high-volume manufacturing techniques, thereby reducing costs. Finally, portable cells allow maintenance staff to quickly replace inoperable cells with pre-tested replacements, reducing the mean repair time.

The SLAC P1 Marx operates in air, has no output transformer, and is air-cooled as shown in Figure 2.21. The Marx utilises a field programmable gate array based control system. A diagnostic module on each cell, along with the ground station and a cell control board, coordinates the timing of the cells. The diagnostic card has four analog input channels monitored at 20 kilosamples per second with a resolution of 16 bits. A fast transient recorder can also be used at 30 megasamples per second with 8-bit resolution. In the SLAC P1 Marx, 16 11-kV Marx cells are arranged with a single 'Vernier' Marx. 
The triggering sequence of the main Marx cells is designed to promptly turn on eleven cells, then stagger the turn-on of the remaining five cells to coarsely compensate the storage capacitor droop. The Vernier Marx (with cells charged to around $1 \mathrm{kV}$ ) staggers its turn-on and turn-off to further regulate the output to the specified level of $\pm 0.5 \%$.
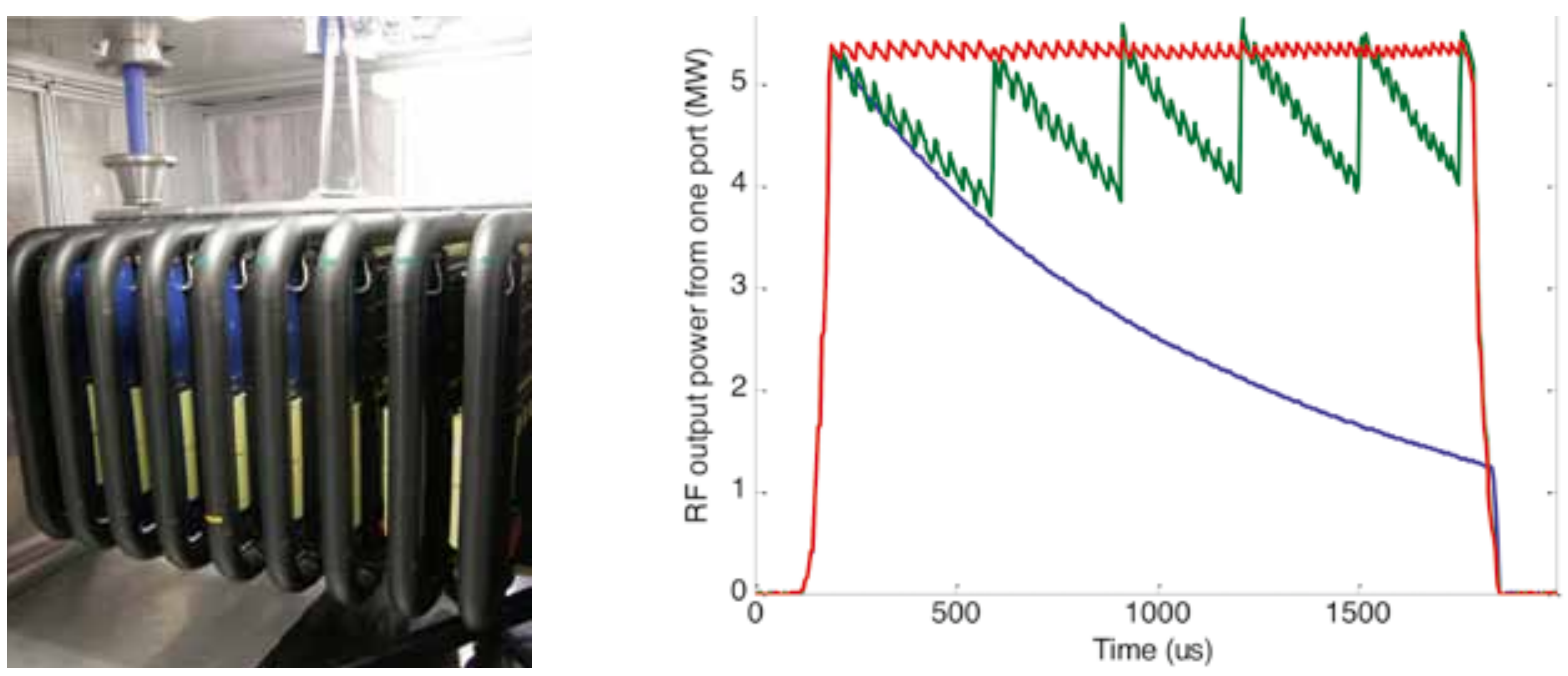

Figure 2.21 Left: SLAC P1 Marx modulator showing its cantilevered support structure, high-voltage grading rings, and 16 installed cells. Right: RF waveforms from one of the two klystron ports: no droop compensation (blue), with only delay cells (green), with delay cells and Vernier (red), producing a flat pulse with a $3 \%$ saw-tooth pattern.

Building upon the success of the P1 Marx, the SLAC P2 Marx is currently in the final stages of design. It includes $323.75-\mathrm{kV}$ to $4-\mathrm{kV}$ cells. This modulator will be able to produce the specified power with up to two of the cells offline. Three notable differences distinguish it from P1. First, a nested droop correction scheme is employed in the P2 Marx. Each cell individually regulates its output, removing the need for a separate compensation element (like the Vernier in the P1 Marx). Second, there is no arraying of solid-state switches within a cell, simplifying the control and protection schemes. Third, the modulator layout is redesigned to have a single-side access.

\section{Main waveguide and tap-offs}

For low transmission loss and robustness against RF breakdown, an overmoded circular waveguide operated in the TEO1 mode is used as the main high-power RF conduit; the attenuation drops faster with radius for this mode than for others, and it has no electric fields terminating on the wall. The diameter chosen is $0.480 \mathrm{~m}$, at which the added ohmic transmission loss along a KCS should be around 6.5\%. With power levels on the order of $300 \mathrm{MW}$, this main transmission waveguide will likely be evacuated and thus needs sufficient wall thickness, about $1 \mathrm{~cm}$. Mode conversion considerations suggest radius, roundness and alignment tolerances on the order of a millimetre and a straightness tolerance of half a degree. The flange joint between pipe sections will be designed to include sufficient longitudinal flexibility to take up local thermal expansion while maintaining concentricity and straightness. An insulation jacket and water cooling will be used to keep much of the main waveguide's heat load (averaging around $130 \mathrm{~W} / \mathrm{m}$ ) from the tunnel air. 
An overmoded waveguide is not trivial to manipulate, as geometrical changes like bends tend to scatter power into parasitic modes. Each main waveguide will need to undergo probably three 9o-degree bends, downward into the shaft, then towards and along the tunnel. These occur at maximum power between the last tap-in and the first tap-off, and will unavoidably have surface electric fields, so the design will be a challenge. They must have excellent port-to-port mode preservation and high-power handling without being excessively bulky. $\mathrm{TE}_{\mathrm{O} 1}$ mode bends exist at X-band, and design options for KCS are currently under consideration.

For tapping off RF power from the main circular $\mathrm{TE}_{\mathrm{o} 1}$-mode waveguide without breaking the azimuthal symmetry and thus introducing surface electric fields in the very high-power region, a special waveguide component was designed, referred to as the coaxial tap-off [2-42]. The idea of this device involves stepping up the diameter from below to above the $\mathrm{TE}_{\mathrm{O} 2}$ cut-off in such a way that roughly half the power is converted, creating a mixture of the two modes. The radial distribution of the electric field then varies longitudinally as the two modes beat as a result of their different guide wavelengths. At an appropriate distance, this beating is terminated by reintroduction of a wall at the original radius, separating the inner volume from a coaxial outer volume and dividing the power between the two in circular and coaxial $\mathrm{TE}_{\mathrm{o} 1}$ modes.

To then extract the stripped power, the coaxial guide is shorted, and power is coupled through eight radial apertures into a wraparound waveguide and thence through two standard WR650 rectangular output ports as shown in Figure 2.22. The gap between the step and the dividing wall is varied over roughly $14 \mathrm{~cm}$ to achieve the many different fractional power couplings needed, ranging between 0.03 and 0.5 , and a small customised ridge before the step is used to cancel any mismatch. The coaxial and wraparound region should never see more than about $10 \mathrm{MW}$, whereas the power in the inner region tops $300 \mathrm{MW}$. If the KCS is evacuated, a 5-MW pillbox window on each rectangular coaxial tap-off port will transition to the pressurised distribution waveguide.
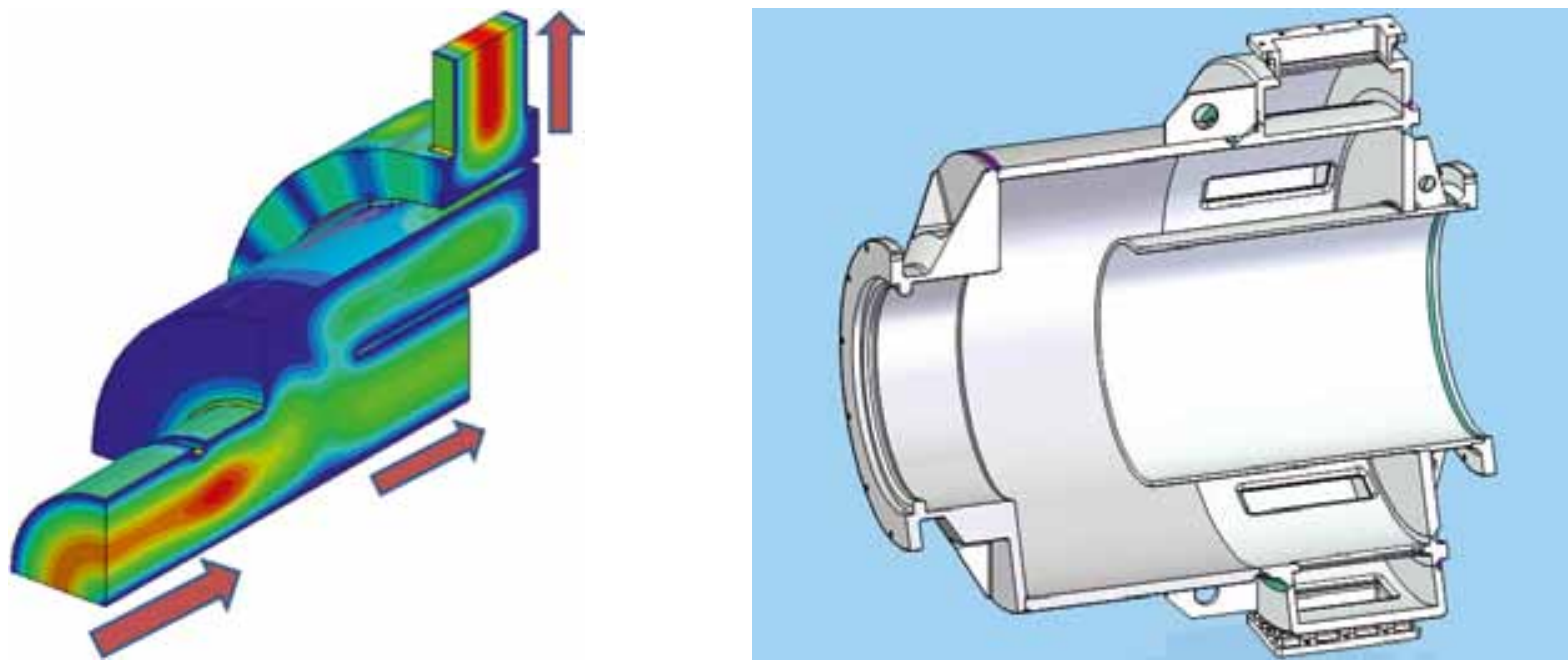

Figure 2.22 Left: simulation model with field plots (electric on cut plane, magnetic on surface). Right: mechanical design of a 3-decibel coaxial tap-off. For other couplings, only the gap length and matching ridge are modified. 
In the surface building, tap-offs are also used in reverse for combining. At each one, proper phasing and relative amplitude ratio between the power flowing in the circular waveguide and the klystron power being added must be achieved for efficient combining. The diameter of the tap-offs' circular ports is smaller than that of the main waveguide. The two are interfaced by a matched double step taper at the end of the combining assembly and before and after each linac coaxial tap-off. These steps, as well as steps and edges in the coaxial tap-off, are radiused to ease pulsed heating.

A pair of 3-decibel ( $\mathrm{dB}$ ) coaxial tap-offs has been fabricated, as have been shorting caps which can be used as launchers, two diameter step tapers, a vacuum pump out spool and four 2.44-m sections of the $0.480-\mathrm{m}$ diameter waveguide as shown in Figure 2.23. A back-to-back cold test of the tap-offs showed good transmission. High-power tests underway are aimed at resonating the waveguide to achieve field levels equivalent to a 300-MW travelling wave and demonstrating transmission at the 4- to 5-MW level available from our test setup's Thales klystron [2-42]. Initial tests with 14.5-pounds-force per square inch gauge (psig) nitrogen pressurisation are being done in the hope that evacuation may not be necessary after all. However, such a conclusion would have to wait until a bend, the likely bottleneck, is so tested.
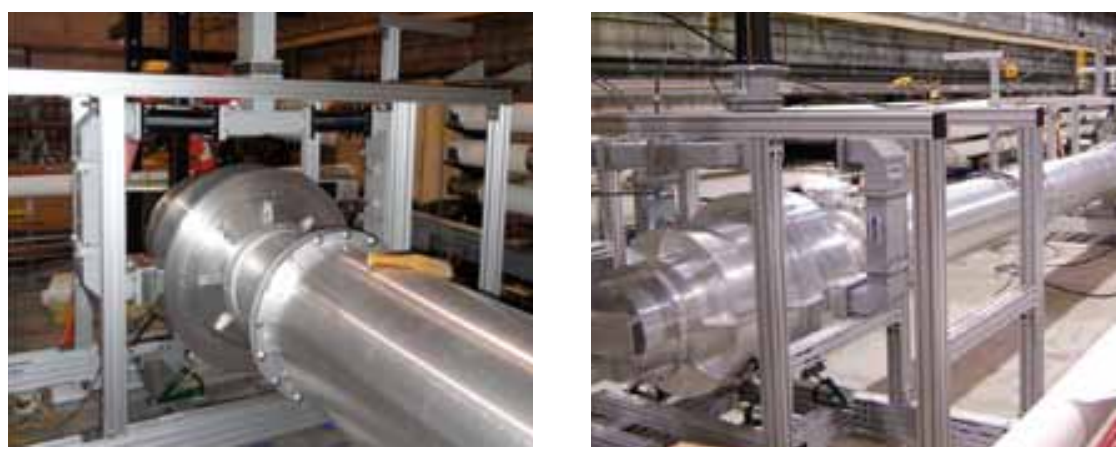

Figure 2.23 KCS R\&D hardware. Left: coaxial tap-off fed through a waveguide $T$ and connected through a taper to the $0.480-\mathrm{m}$ diameter circular waveguide. Right: a four-section 10-m run of the KCS main waveguide.

\section{Local waveguide power distribution system}

The RF power extracted from each coaxial tap-off along the linac tunnel is distributed to 26 cavities in three cryomodules (9-8-9, with a quadrupole magnet in the centre cryomodule), constituting an RF unit. The waveguide power distribution system (PDS) through which it flows has evolved since the RDR, as shown in Figure 2.24. The three-stub tuner has been eliminated, its dual functions now accomplished by phase shifters with a movable side wall and tunable coaxial fundamental power couplers, both of which are motorised. Feeding properly spaced cavities in pairs through a 3-dB hybrid allows the combined reflected power to be directed to a load on the hybrid's fourth port. Thus the expensive circulators can likely also be eliminated for most cavities. Finally, with the wide range of sustainable ILC cavity gradient limits $( \pm 20 \%)$ now to be accepted to increase production yield, power efficiency demands we tailor the distribution to the (sorted pairs of) cavities in each RF unit. Thus adjustable coupling from the main WR650 waveguide is included. This latter function can be provided by a novel waveguide component developed at SLAC called the variable tap-off [2-43]. 
This adjustable directional coupler works by means of mode rotation of a circular $\mathrm{TE}_{11}$ mode accomplished via physical rotation of an oval middle section. A PDS incorporating four vertical tap-offs was built and high-power tested for the first cryomodule that will be tested at Fermilab [2-44].



Figure 2.24 Left: updated modular PDS waveguide layout. Power for two cavities at a time is coupled from a main WR650 feed and divided through a hybrid. Reflections from the cavities combine into the hybrid load, allowing elimination of circulators (included after the hybrid in the 3-D view), except for the odd cavity in a nine-cavity cryomodule. Right: one of four PDS modules built and tested at SLAC for the first cryomodule of Fermilab's L-band NML test accelerator.

\subsubsection{Distributed RF scheme}

\section{Basic concept of DRFS compared with the RDR}

The distributed RF scheme (DRFS) was proposed as another possible costeffective solution for a single main linac tunnel design in the proposal of SB2009 [2-45]. The basic concept of DRFS is illustrated in Figure 2.25. The salient feature of DRFS is a complete single-tunnel plan with no highpower RF components on the surface, utilising approximately 8,0oo small modulating-anode (MA) klystrons of 8oo-kW output power, driven by DC power supplies and MA modulators. The RF power for two superconducting cavities is fed by a klystron through a rather simple PDS without employing a circulator. By contrast, the RDR presented a two-tunnel plan in which a 10-MW multi-beam klystron feeds power to 26 cavities through a complicated PDS using 26 circulators in RDR RF units comprising three cryomodules. 


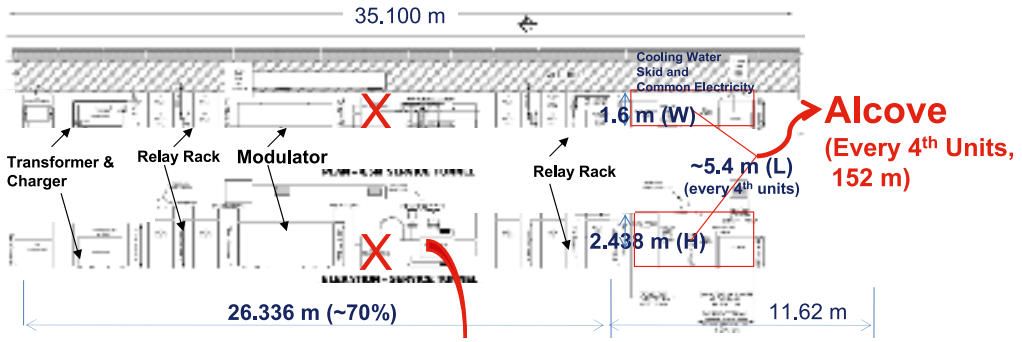

Figure 2.25 Schematic layout

of the distributed RF scheme.

One big HV klystron replaced by 13 small klystrons

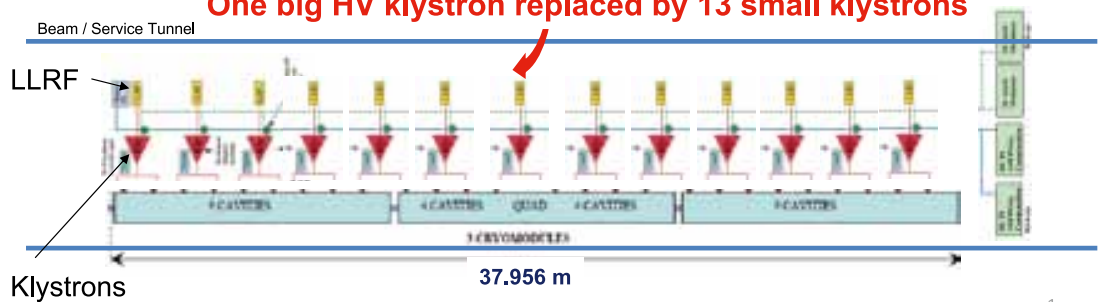

Klystrons

$37.956 \mathrm{~m}$

The advantage of the DRFS is that it represents a complete single-tunnel plan in which there are no high-power RF facilities on the surface. One klystron feeds power to only two cavities, facilitating optimised cavity performance. In the case of local superconducting cavity failures, it is easy to separate them from the operation, giving DRFS good operability. There is a small probability of big failures that prevent beam operation. There are also concerns about the construction cost, maintainability and heat loss problems, since all heat loads are also in the single underground tunnel (this will be described in a later section). Though it uses mature technology, DRFS is a newly proposed plan. The technical feasibility is being demonstrated in part of the S1-Global programme at KEK.

\section{DRFS configuration in the tunnel}

Since DRFS is a complete single-tunnel plan, its configuration strongly depends on the tunnel shape and the layout of cryomodule in the tunnel. If the tunnel cross-section is assumed to be circular, constructed by tunnel boring machine, two configurations are possible: in the first configuration the cryomodule hangs from the ceiling; in the second, the cryomodule is installed on the floor. The current likely configuration of DRFS is based on the latter, which is shown in Figure 2.26. The diameter of the tunnel cross-section is $5.7 \mathrm{~m}$. The tunnel is divided into three horizontal regions: a cryomodule region, a passage and maintenance space including egress and a region of high-power RF equipment, separated from the other regions by a radiation shielding wall with access doors. It is shown that all required components, including standby power supply, are in the highpower equipment region. Waveguides of the PDS are laid under the floor. The tunnel is also divided into three regions in the vertical direction and accommodates all required functions, including water supply, ventilation and the space to exhaust an accidental helium leak. It is necessary to investigate this configuration in more detail to assure sufficient working space during installation and maintenance, and this will be done after optimisation of the RF component sizes. 

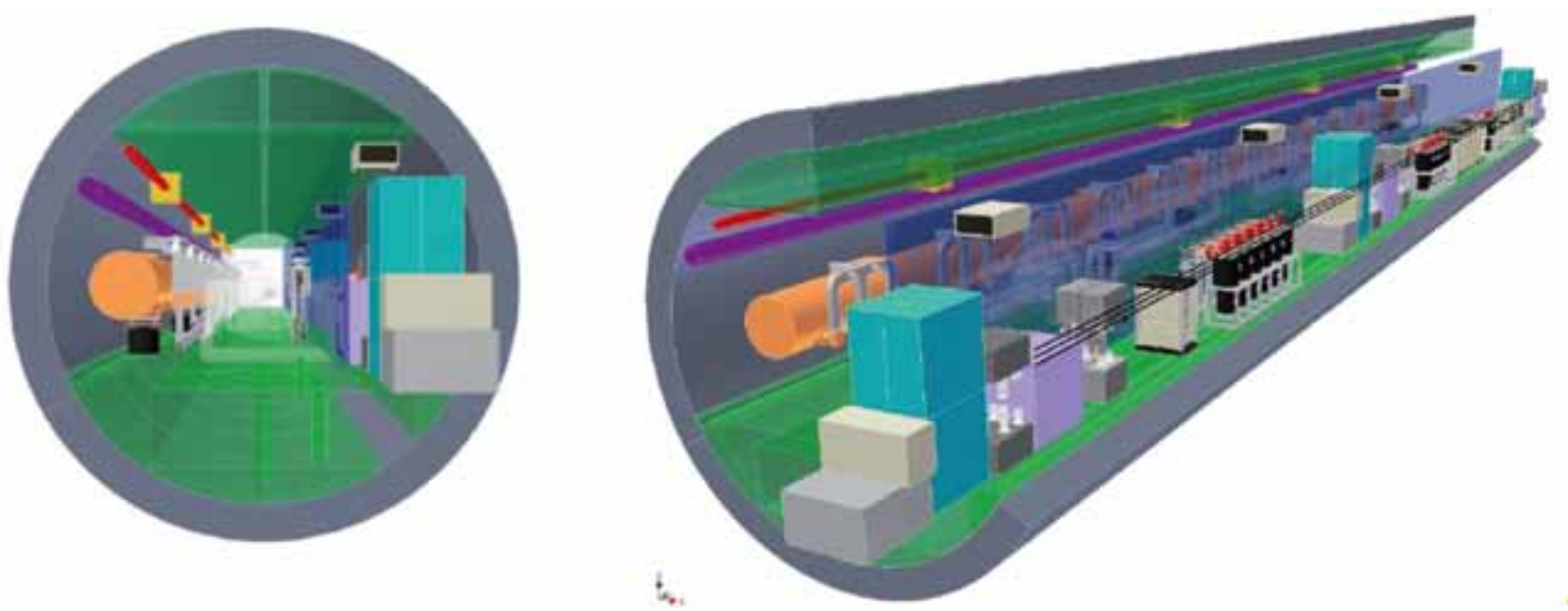

Figure 2.26 DRFS configurations in the tunnel shown in 3-D. Left: cross-section view. Right: three-quarters view.

\section{Operability and availability of DRFS}

Since DRFS can control two superconducting-cavity units with low-level RF, operability is better than foreseen in the RDR. Some of the advantages of the DRFS have already been described in an earlier section. Thirteen DRFS klystrons are operated on a common DC power supply and MA modulator, so the required applied high voltage is determined by the maximum power in the unit, if a fixed overhead for low-level RF control is assumed. From superconducting cavity manufacturing, a $20 \%$ variation in the accelerating gradient of $31.5 \mathrm{MV} / \mathrm{m}$ should be accepted, and in order to achieve an efficient system, sorting of the cavities is inevitable for DRFS. Assuming that 3,400 cavities are manufactured every year in the construction period and that they are sorted in bins with an $8 \%$-range variation of field gradient, the resulting number in a bin is 680 . These are sorted in an RDR unit (three cryomodules) with $4 \%$ higher power without sacrificing the field gradient. Therefore in DRFS, this kind of cavity sorting is prescribed. In order to achieve high availability in DRFS, we assumed 110,00o hours of mean time before failure for MA klystrons and introduced backup DC power supplies and MA modulators. This configuration is shown in Figure 2.27 , in consideration of the DC power supply size and cost. In this case, the basic configuration of the DRFS baseline (or the high-current case) includes two regular DC power supplies and MA modulators plus another backup in two RDR units. So far, for other availability and installation issues, the basic scenario is kept to be the same as SB2009, as long as we employ the configuration of Figure 2.27. 
Availability Consideration Revised



Full Power Option@26-Cavities (1 klystron feeds 2 cavities)

- 1 DC Pis +0.5 Back-up

1 MA Puiser +0.5 Back-up

13 Kiylutons

26 Cavies

13 Magionee(tybidi)
DC AS tor 13 Nepritors

WA Moddatm Sor 13 Nintera

und

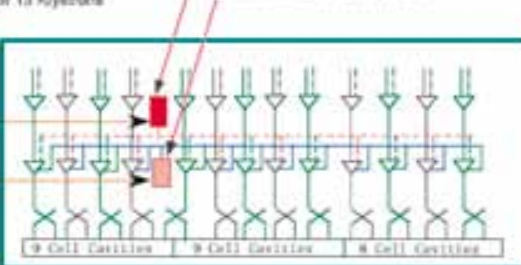

Full Power Option@ 26-Cavities

( 1 klystron feeds 2 cavities)

$=1$ DC Pis +0.5 Bach-up

1 Ma Puser +0.5 llack-up

13 Klystrons

26 Caviles
Figure 2.27 Two-RDR-unit system of DRFS in

the baseline case is shown. Red boxes represent

the DC power supplies. Pink boxes represent

modulating-anode modulators for active components.

Blue boxes represent the DC power supplies for

backup components. Light blue boxes represent

the modulating-anode modulator for backup

components.

Another important issue for DRFS is radiation. There is a concern about semiconductor damage from the radiation in the high-power RF components. As shown in Figure 2.27, DRFS employs a radiation shield wall which has the same shield thickness investigated by DESY; it is planned to investigate the effect more precisely.

\section{R\&D status of DRFS}

A DRFS demonstration to show feasibility is planned at KEK to extend over three consecutive years. The first demonstration is scheduled at the beginning of 2011. It will employ a two-unit DRFS in the S1-Global project in KEK [2-46]. The first prototype DRFS klystrons, a power supply and an MA modulator will be installed and operated to feed power to four cavities. Low-level RF control is a main R\&D theme to evaluate the feedback technology when there are no circulators.

Prototype klystrons of DRFS were manufactured in 2010, and factory tests satisfied the required original specification: $750 \mathrm{~kW}$, as shown in the left image of Figure 2.28. The output power is increased to $800 \mathrm{~kW}$ to accept the gradient variation of $32.5 \mathrm{MV} / \mathrm{m} \pm 20 \%$; factory tests cleared this revised specification. Output characteristics of prototype klystrons are shown in the right image of Figure 2.28. The results for first klystron are an output power of $813 \mathrm{~kW}$ at $64.2 \mathrm{kV}$ with an efficiency of $57.4 \%$ for micro-perveance of 1.36 and output power of $806 \mathrm{~kW}$ at $67.1 \mathrm{kV}$ with an efficiency of $60.1 \%$ for microperveance of 1.15. Since the DRFS klystron is an MA klystron, beam perveance can be controlled by the ratio between cathode-to-MA electrode voltage and cathode-to-anode voltage. A prototype power supply and MA modulator have been delivered to KEK and are operated in an acceptance test. All DRFS high-power components were moved near to the cryomodule in the S1-Global tunnel and operated as the demonstration at the end of 2010. The configuration of test arrangement in S1-Global is shown in Figure 2.29. 

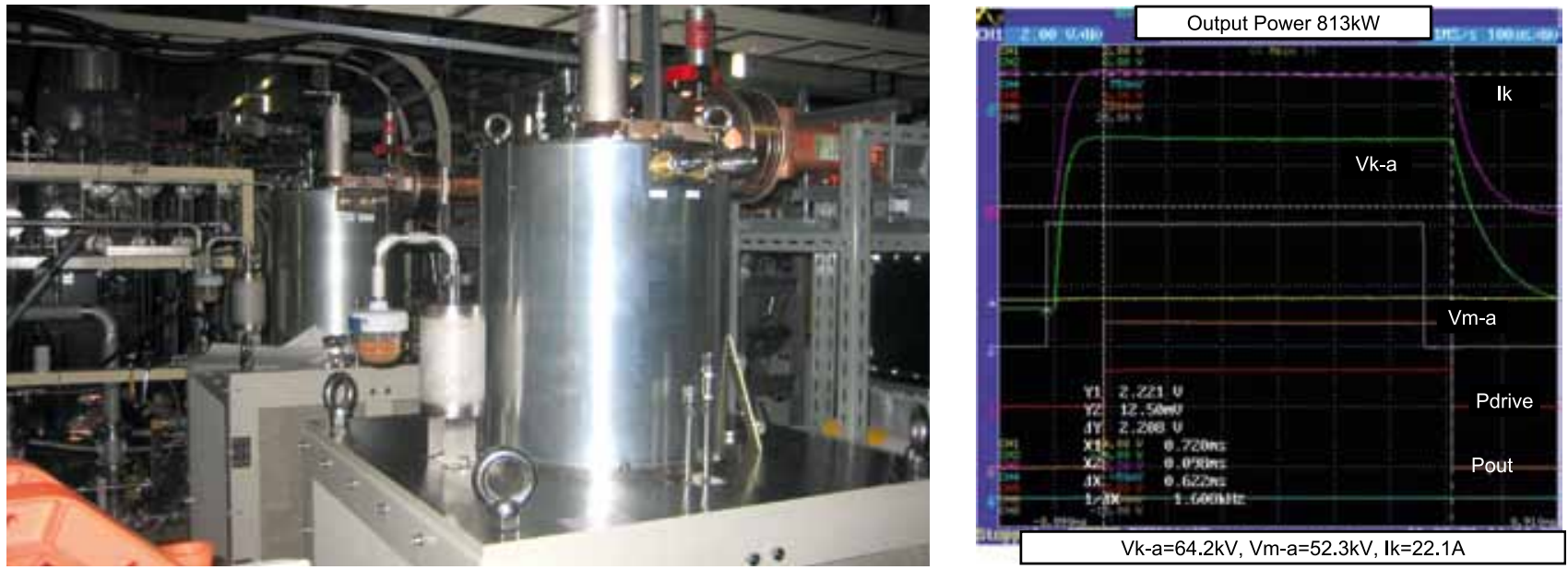

Figure 2.28 Klystrons (left) and waveforms in the test (right).

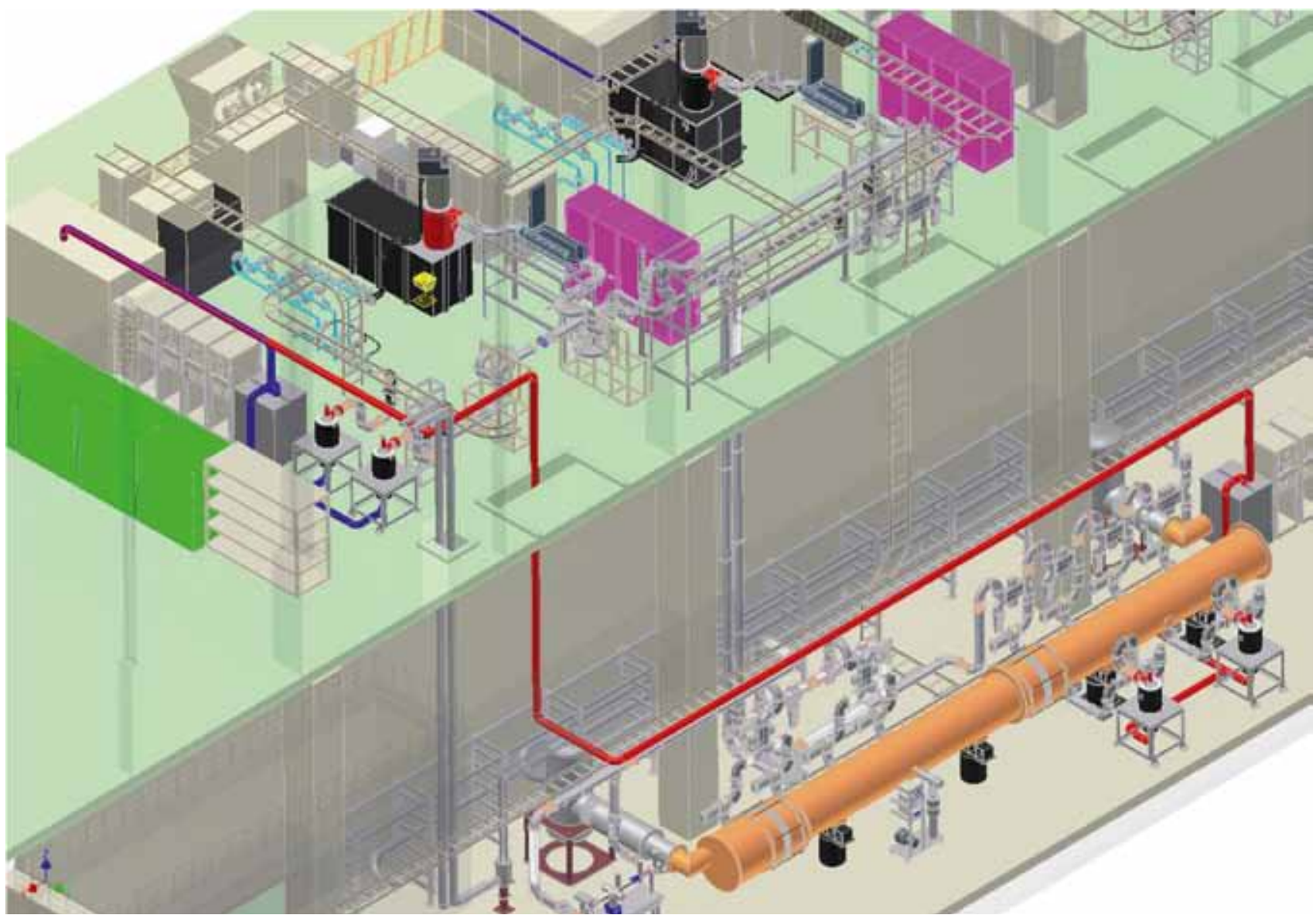

Figure 2.29 Layout of the DRFS test configuration in S1-Global. At first, high-power RF components are tested and evaluated on the first floor with a matched load on the PDS (shown in blue), and then systems are moved near the cryomodule in the tunnel (shown in red). 
The second demonstration is a continuation to check the long-term stability by installing one of the above DRFS units in the 'quantum-beam project' at KEK. The third demonstration, planned for the years 2012 to 2013, entails the installation of a four-unit DRFS feeding RF power to eight cavities in the STF phase 2 project at KEK. During these R\&D schedule periods, key elements of the DRFS high-power system components will be developed, including spark gap development in the crowbar circuit, high-voltage relay in the DRFS power supply system and permanent magnet R\&D for the klystron. Some of this work has been already begun in collaboration with industry. Since the DRFS concept is well established and employed in many projects, we think there are no basic difficulties, but some problems need to be solved for system installation. The purpose of the R\&D should be focused in this direction. $R \& D$ for cost reduction is another very important issue, and after successful operation, design efforts to reduce the cost of each component are strongly required.

\subsubsection{RDR configuration in single tunnel as backup}

SB2009 demands a single-tunnel configuration for the ILC main linacs in order to reduce project cost. Because the options described in the preceding two sections are in the R\&D stage, a backup plan was put forward in which the equipment of the RDR high-power RF system, which is better studied, is incorporated into the linac tunnel. One possible RDR single-tunnel configuration is almost identical to that of the European XFEL, with pulse transformers, 10-MW multi-beam klystrons and power distribution systems in a single tunnel of a diameter of $5.2 \mathrm{~m}$ and modulators on the surface. Another single-tunnel plan for the RDR system has all high-power components in a single tunnel of a diameter of $6.5 \mathrm{~m}$. For a mountainous site, this type of complete single-tunnel plan is preferable. The pros and cons of this latter option are largely shared with DRFS, since both are complete single-tunnel plans.

\subsubsection{Global progress in cavity and cryomodule string integration and tests} The cavity/cryomodule string integration and cold tests have made global progress in three major facilities of TESLA Technology Collaboration/FLASH at DESY in Europe, the New Muon Laboratory (NML) at Fermilab in the Americas region, and the Superconducting Test Facility (STF) at KEK in Asia [2-47]. TTC/FLASH has made much progress with beam acceleration by using a series of cavity/cryomodule strings [2-48]. NML construction started in 2007, and the first cryomodule installation and the associated facility was completed in 2010 [2-49]. The RF test is being prepared. STF construction start was in 2005, and the first cryomodule test was performed in 2007 without beam. Since spring 2010, the S1-Global cryomodule test has been carried out as a global cooperation programme [2-50]. Table 2.7 summarises progress and plans for these facilities.

\subsection{SYSTEM INTEGRATIONS TESTS}


Table 2.7 Progress in cavity/cryomodule integration and tests for TTF/FLASH, NML, and STF.

\begin{tabular}{|c|c|c|}
\hline Location & Year & Progress \\
\hline \multirow{5}{*}{$\begin{array}{l}\text { TTF/ } \\
\text { FLASH } \\
\text { (DESY) }\end{array}$} & 2005 & TTF2/FLASH integration and test started \\
\hline & 2008 & ILC 9-mA beam: first beam with 3-mA, 500-ms beam pulses \\
\hline & 2009 & Operation with high-power ILC-like beam with 22-kW average power \\
\hline & 2011 & Gradient-margin studies with long beam pulses \\
\hline & 2012 & Studies of beam operation at the limits of gradient and RF power \\
\hline \multirow{4}{*}{$\begin{array}{l}\text { NML } \\
\text { (Fermilab) }\end{array}$} & 2007 & NML first cryomodule integration \\
\hline & 2010 & Integration completed and cool-down started \\
\hline & 2012 & Planned: NML accelerator system integration to be complete \\
\hline & 2013 & Planned: Beam acceleration to start \\
\hline \multirow{5}{*}{$\begin{array}{l}\text { STF } \\
\text { (KEK) }\end{array}$} & 2007 & STF S-1: cavity/cryomodule system integration and test \\
\hline & 2010 & S1-Global: cryomodule assembly and cold test \\
\hline & 2011 & Planned: quantum beam integration and beam test to start \\
\hline & 2012 & Planned: STF-2 accelerator system integration to be complete \\
\hline & 2013 & Planned: STF- 2 beam accelerator to start \\
\hline
\end{tabular}

\subsubsection{Cavity/cryomodule string integration and test at NML, Fermilab} Fermilab is currently constructing the SCRF Test Accelerator at the New Muon Lab as described in section 2.4. The initial primary purpose of NML will be to test superconducting RF accelerating cryomodules for the ILC and for Fermilab's Project X, a proposal for a high-intensity proton source. The unique capability of NML will be to test these modules under conditions of high-intensity electron beams with ILC-like beam parameters. In addition NML incorporates a photo injector, which offers significant tunability and the possibility of generating an electron beam with a brightness comparable to state-of-the-art accelerators. This opens the exciting possibility of also using NML for fundamental beams research and tests of new concepts in beam manipulations and acceleration, instrumentation and the applications of beams.

Building infrastructure - cryogenics, electrical power, RF power, water cooling, electronics racks, shielding - is currently being installed. A single superconducting cavity is currently installed and tested, and a single SCRF cryomodule (type TTF III+) is being cooled down since November 2010. Beamline construction will start in late 2011 and we expect to start delivering beam in late 2012.

The NML injector beamline is shown in Figure 2.30. It consists of a 1.3-GHz RF photo-emitted electron gun, followed by two SCRF accelerating cavities, a bunch compressor and beam diagnostics. The primary injector beamline will operate at around $4 \mathrm{O} \mathrm{MeV}$ and is some $22 \mathrm{~m}$ long. It will be capable of producing an ILC-like beam structure with a bunch charge of 3.2 nanocoulombs $(\mathrm{nC})$, a 3-MHz bunch repetition rate, a bunch train length of $1 \mathrm{~ms}$, a $5-\mathrm{Hz}$ bunch train repetition rate, and peak current in excess of $10 \mathrm{kA}$. The single bunch charge can be well over $20 \mathrm{nC}$. In addition, there is floor space for two reconfigurable $4 \mathrm{O}-\mathrm{MeV}$ test beamlines for a variety of experiments. 


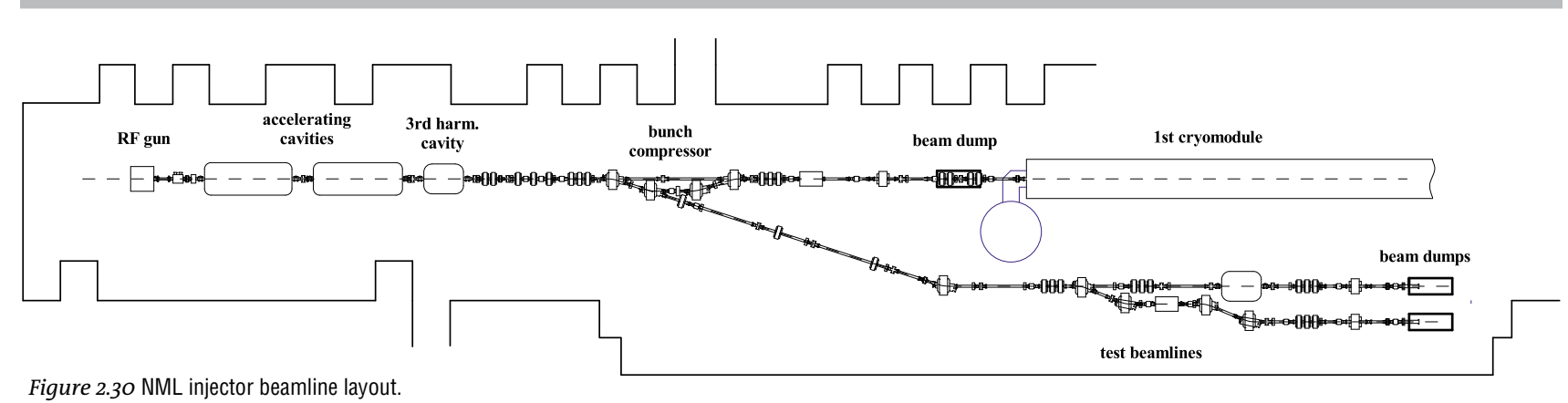

Figure 2.30 NML injector beamline layout.

The acceleration section will initially consist of three ILC-type SCRF cryomodules (a single ILC RF unit powered by a single 10-MW klystron) capable of accelerating beam to around $750 \mathrm{MeV}$. A building expansion, almost completed, will allow for a total of up to six cryomodules and up to $1,500 \mathrm{MeV}$ of beam energy. A plan for the high-energy downstream beamlines is shown in Figure 2.31. There will be floor space and infrastructure available for up to three high-energy test beamlines (18 to $34 \mathrm{~m}$ in length) and a storage ring up to $10 \mathrm{~m}$ in diameter. High-energy beam dumps are being designed to absorb the $75 \mathrm{~kW}$ of beam power.

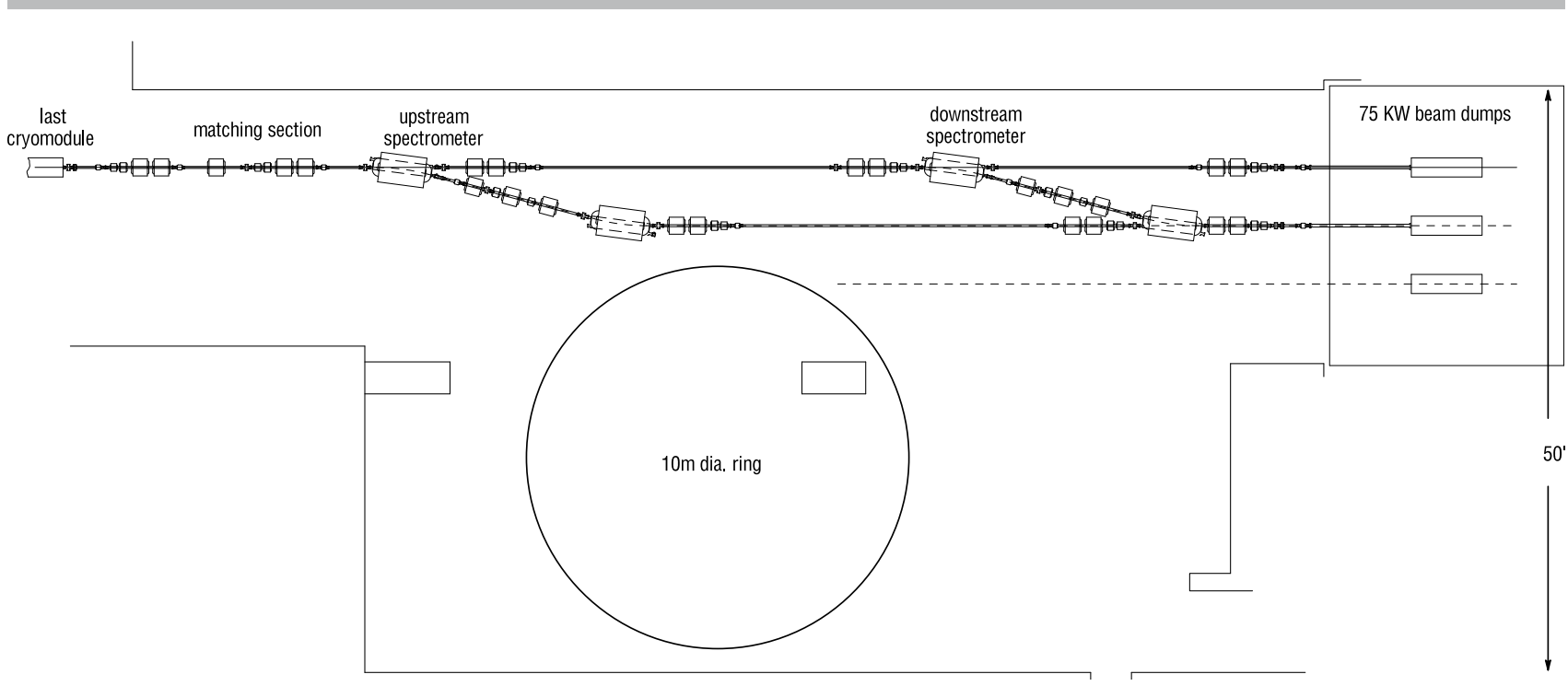

Figure 2.31 High-energy beamline layout at NML.

In summary, in addition to providing realistic tests of a new generation of RF cryomodules, the new NML facility offers excellent opportunities to advance ILC system integration tests, as well as accelerator science and technology on several fronts. Eventually, NML will become a truly open user facility with unique capabilities to advance accelerator research by groups from various institutions, to enhance accelerator education and to promote accelerator technology development for industrial applications. 


\subsubsection{Cavity/cryomodule integration and string test at KEK's STF}

ILC cryomodule development, test and accelerator integration activities are done in STF at KEK. An accelerator tunnel of $100 \mathrm{~m}$ length is used for cryomodule test and system integration. The three high-power RF power stations and the cryogenics plant for the cryomodule are located in the nearby surface building, which measures $60 \mathrm{~m}$ by $30 \mathrm{~m}$.

\section{STF-1 Cavity/cryomodule test}

The STF phase 1 test cryomodule consists of two 6-m horizontal cryostat units of half the length of the ILC design. Each of them can accommodate four cavities. The type-A cryomodule is designed to accommodate TESLA-like cavities, and the type-B cryomodule is for low-loss cavities [2-51]. A cool-down test was carried out for the TESLA-like cavities in Cryomodule-A and one low-loss cavity in Cryomodule-B in early 2008. Another cool-down test using four TESLA-like cavities in Cryomodule A followed in 2008. One cavity out of the four reached 31.5 MV/m, the nominal ILC operational gradient. The other three stayed around $20 \mathrm{MV} / \mathrm{m}$. A study of Lorentz force detuning measurement and compensation was made, and the stabilisation of field amplitude and phase of cavities was demonstrated by digital feedback control using piezo actuator Lorentz force detuning compensation. An amplitude and phase stability of $0.04 \%$ root mean squared (rms) and o.o2-degree rms, respectively, was demonstrated. This stability performance is well within the ILC specification. Several tests of low-level RF and power distribution were performed, including simulated beam loading signal mixture, special filtering techniques and intermediate-frequencymixture analogue-to-digital converter detection, as well as loaded Q-value control using waveguide shorts and phase shifters.

\section{S1-Global cavity/cryomodule test}

The primary goal of the S1-Global programme is the 'realisation of an average accelerating gradient of $31.5 \mathrm{MV} / \mathrm{m}$ with eight cavities' in the S1-Global cryomodule, which is composed of components contributed by the different collaborating partners [2-47,52].

The general plan for the S1-Global programme is summarised in Table 2.8. The S1-Global cryomodule consists of two 6-m cryomodules, Cryomodule-A and Cryomodule-C, shown in Figure 2.32. Four cavities from Fermilab and DESY are installed in Cryomodule-C, and four cavities in two different kinds of cavity jackets developed by KEK are installed in Cryomodule-A. The parameters of the two 6-m cryomodules are listed in Table 2.8. The contributions of the participating laboratories demonstrate the collaborative framework of S1-Global:

- DESY: two TESLA-type cavities including Saclay-type tuners and power couplers

- Fermilab: two TESLA-type cavities, power couplers and integration of the INFN blade tuners in the cavity packages

- KEK: four TESLA-like cavities, with two variations of tuner/jacket design, Cryomodule-A for KEK cavities, power distribution for Cryomodule-A, and infrastructure for tests

- INFN: design and construction Cryomodule-C in cooperation with KEK, and production of the blade tuners for the Fermilab cavities

- SLAC: two sets of VTO power distribution for Cryomodule-C, and processing of Fermilab couplers 
In addition to the main target of demonstrating an average accelerating gradient of $31.5 \mathrm{MV} / \mathrm{m}$ with eight cavities during the cryomodule testing, the S1-Global programme has the following R\&D goals:

- The operation at an average accelerating gradient of $31.5 \mathrm{MV} / \mathrm{m}$ in pulsed $\mathrm{RF}$ conditions at $5 \mathrm{~Hz}$ with 1-ms flat-top length under stable conditions: $0.07 \%$ rms amplitude variation and 0.35 -degree $\mathrm{rms}$ phase variation

- Experience with the design, assembly and the alignment procedures for different types of cavity packages from participating parties

- The measurement and comparison of the cryogenic performance for each cavity package and cryomodule (in terms of heat loads to the 2-K environment) in the static and high accelerating field dynamic conditions

- The comparative studies of RF performances of the cavity packages (including tuner concepts) from the participating institutes

- Advancement in the implementation of the plug-compatible concept for the ILC activities
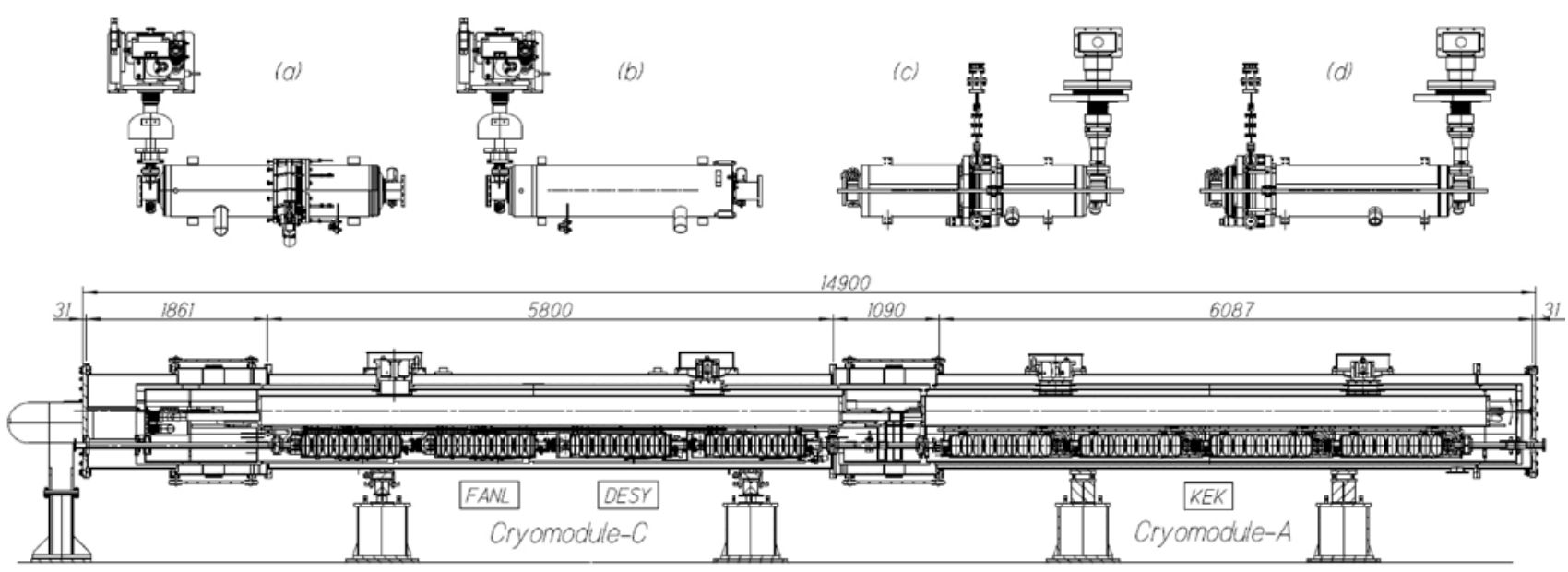

Figure 2.32 S1-Global cryomodule and cavity package of each laboratory. (a) Fermilab cavity with INFN blade tuner. (b) DESY cavity with Saclay-type tuner. (c) KEK-A cavity with slide jack tuner. (d) KEK-B cavity.

\begin{tabular}{|c|c|c|}
\hline & Cryomodule-A & Cryomodule-C \\
\hline Vacuum vessel length & $6,087 \mathrm{~mm}$ & $5,800 \mathrm{~mm}$ \\
\hline Distance b/w couplers & $1,337.0 \mathrm{~mm}$ & $1,383.6 \mathrm{~mm}$ \\
\hline Cavity package & KEK-A/KEK-B & Fermilab/DESY \\
\hline Cavity type & TESLA-like & TESLA-type \\
\hline Tuner type & Slide jack & Blade/CEA \\
\hline Input coupler type & Disk window & Cylindrical window \\
\hline Magnetic shield & Inside jacket & Outside jacket \\
\hline Package length & $1,247.6$ & $1,247.4 / 1,283.4$ \\
\hline
\end{tabular}

Table 2.8 S1-Global cryomodule parameters. 
Figure 2.33 Cooling characteristics of the S1-Global cryomodule with eight cavities in the second cooling test period.

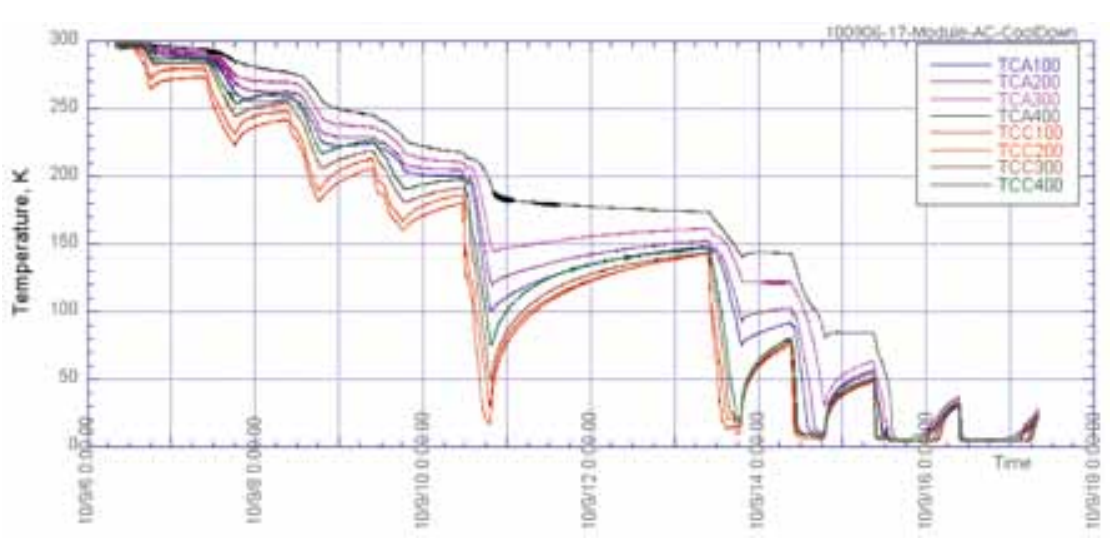

Figure 2.34 Cavity performance in the S1-Global cryomodule. Blue bars show the gradient measured for individual cavity in vertical tests. Red bars show the achieved gradient for each individual cavity in the S1-Global cryomodule test. Green bars show the average gradient for the seven-cavity string test in simultaneous RF operation. The average gradient is $30 \mathrm{MV} / \mathrm{m}, 27 \mathrm{MV} / \mathrm{m}$ and $26 \mathrm{MV} / \mathrm{m}$, respectively.

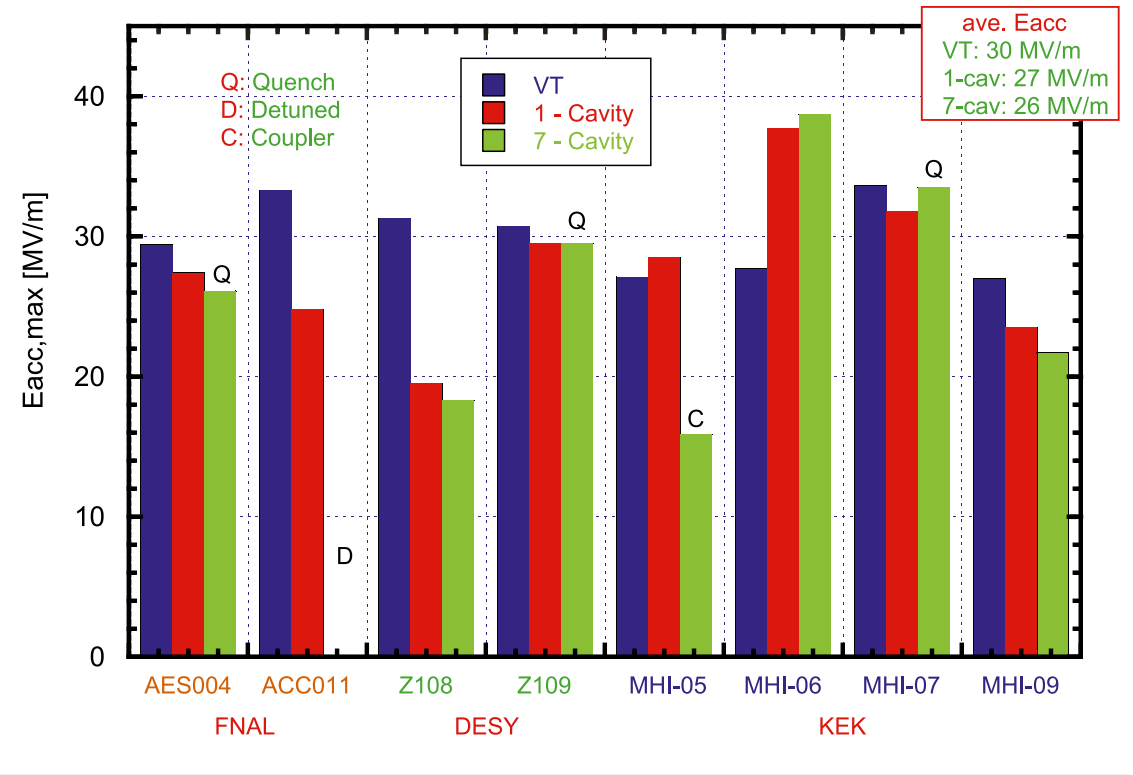

The S1-Global cryomodule has been cooled down to $2 \mathrm{~K}$ twice, as shown in Figure 2.33, and a series of experiments has progressed. The average maximum gradient of individual cavities was observed to be $27 \mathrm{MV} / \mathrm{m}$, and the maximum gradient average in the seven-cavity string operation was observed to be $26 \mathrm{MV} / \mathrm{m}$, compared to the maximum average gradient in each vertical test of $30 \mathrm{MV} / \mathrm{m}$, as shown in Figure 2.34. The Lorentz force detuning characteristics for the four kinds of tuner systems were also measured for each cavity, and a new Lorentz force detuning compensation scheme was tried. The resulting experimental performance is now under analysis. The S1-Global cold test has successfully evaluated thermal performance, tuner performance, cavity high-gradient operation using vector-sum control, as well as Lorentz force detuning compensation, using both the RDR RF scheme and the DRFS system. 


\section{Development plans of STF phase 2}

The planned STF phase 2 accelerator is illustrated in Figure 2.35. The new power scheme of DRFS, which is proposed for single-tunnel design in a mountainous site, will be demonstrated instead of the RDR power scheme.

It consists of two ILC cryomodules driven by nine DRFS klystrons, a photocathode RF gun driven by a 5-MW klystron, and two nine-cell-cavity capture modules driven by a DRFS klystron. The current compact X-ray source development is included in the commissioning of the beam source as a part of the ILC test phase-2 accelerator.

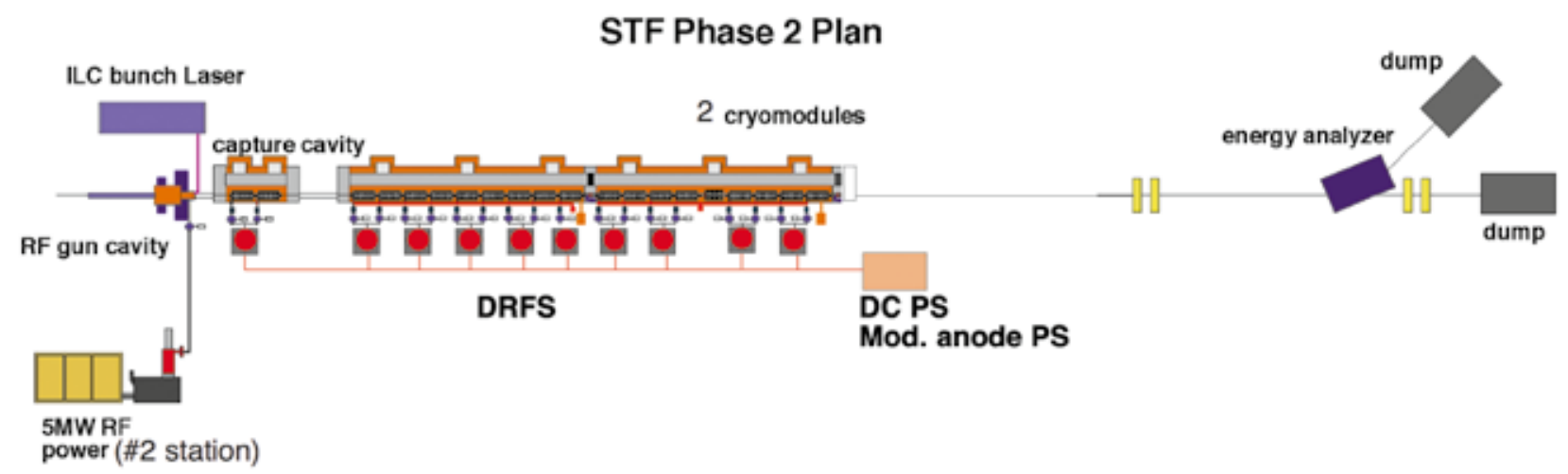

Figure 2.35 General layout of planned STF phase-2 accelerator. The RF gun, the capture cryomodule, two ILC-type cryomodules are aligned. The cavities will be powered by DRFS klystrons, while the RF gun will be powered by a 5-MW klystron. This accelerator is in the STF tunnel, which is $10 \mathrm{~m}$ below the STF building.

The production of two cavities for the capture section and nine cavities for the first cryomodule started in 2009 and will be completed in 2011. The beam source development to build the photocathode RF gun system took a major step forward in 2010. The RF gun cavity and the input coupler were fabricated and delivered to STF by the development of the DESY-Fermilab-KEK team through the US-Japan collaboration programme in November 2009. After minor modifications to the cavity water jacket and beam pipe flange, the pumps, solenoid, waveguide, RF window and the Cs2Te photocathode system were assembled into the gun cavity. RF processing of the cavity was performed up to 1.7-MW input without serious problems. The drive laser for the photocathode was also developed and delivered from the Institute of Applied Physics (IAP) in Nizhny Novgorod, Russia and the Joint Institute for Nuclear Research (JINR) in Dubna, Russia. The laser system is a collaborative development between KEK, IAP and JINR, begun in 2007. The laser generates 10-picosecond pulsed UV light $(266 \mathrm{~nm})$ with 3-MHz repetition during a 1-ms RF pulse with $5-\mathrm{Hz}$ klystron repetition. The extracted electron charge in one bunch is designed to be $3.2 \mathrm{nC}$, which is the ILC specification. After finishing the X-ray generation experiment, the first ILC cryomodule will be installed in the STF tunnel by the end of 2012. The second run of the phase-2 accelerator is scheduled from January 2013 to July 2013. Parallel to this, a second cryomodule will be in preparation. 


\subsubsection{The TTF/FLASH ' 9 -mA' experiment at DESY}

The ' 9 - $m A$ ' programme was proposed by the GDE in 2008 and subsequently taken up by DESY with a view to performing system tests in support of both the ILC S-2 beam test programme and the European XFEL development. Operationally, the programme is led by DESY, while the scientific programme is coordinated jointly by DESY and the GDE. International participation in the programme, notably from ANL, Fermilab and KEK, has concentrated on low-level RF and accelerator controls [2-48].

The primary goal for TD Phase 1 was to demonstrate reliable operation of the TTF/FLASH linac with ILC-like high-power beams. Other specific goals are:

\section{Long bunch train high beam loading demonstration}

- 8oo-ms pulse: 2,400 bunches at $3 \mathrm{MHz}, 3 \mathrm{nC}$ per bunch

- vector sum control of up to 24 cavities

- +/- 0.1\% energy stability

- beam energy from 700 to $1,000 \mathrm{MeV}$

\section{Study operation at the limits}

- determining energy stability limitations and trade-offs

- cavity gradient margins and RF power overhead

These goals broadly address linac integration and operation with ILC-like beams. Aside from the primary demonstration of reliable operation under ILC-like conditions, the critical R\&D task is to assess operational requirements for gradient-margin and RF-power overhead, which provide direct input to the ILC main linac design parameters. In the following sections we will briefly describe the FLASH facility, results and accomplishments from the two main beam studies periods to date, and give an outlook for future 9-mA studies.

\section{FLASH overview}

The TESLA Test Facility at DESY was constructed by the international TESLA collaboration to demonstrate the feasibility of a linear collider based on superconducting accelerating structures of high performance and cost-competitiveness with conventional copper structures. Technical feasibility of superconducting accelerating structures was demonstrated in 2000 when an 800-ms-long 8-mA beam was accelerated through a single cryomodule to $168 \mathrm{MeV}$.

TTF has subsequently been upgraded several times. In 2005, TTF became FLASH (Free-electron laser in Hamburg), began operation as a free-electron laser (FEL) photon user facility and has since accumulated more than 25,000 hours of accelerator operation. The most recent upgrades in $2009 / 2010$ included raising the maximum operating energy to $1.25 \mathrm{GeV}$.

The main elements of FLASH before the 2009/2010 upgrades, shown in Figure 2.36, are a 5-MeV laser-driven photo-cathode RF gun, two stages of bunch compression, and six cryomodules (ACC1 to ACC6), each containing eight TESLA-type 1.3-GHz superconducting RF cavities. Four klystrons supply RF power to the electron gun, eight cavities in ACC 1,16 cavities in ACC23, and 24 cavities in ACC456, respectively. Low-level RF controllers associated with each klystron regulate the vector sum of the fields in each group of cavities. 
The RF unit comprising the 24 cavities in ACC 456 is of particular interest for the 9-mA studies because of the close resemblance to the RF configuration in the ILC reference design. In this configuration, the beam energy at the end of the linac can be as high as $1 \mathrm{GeV}$. Electron bunches are then transported either through a series of undulators for FEL photon generation or through a bypass line, and then are finally transported to a high-power beam dump.

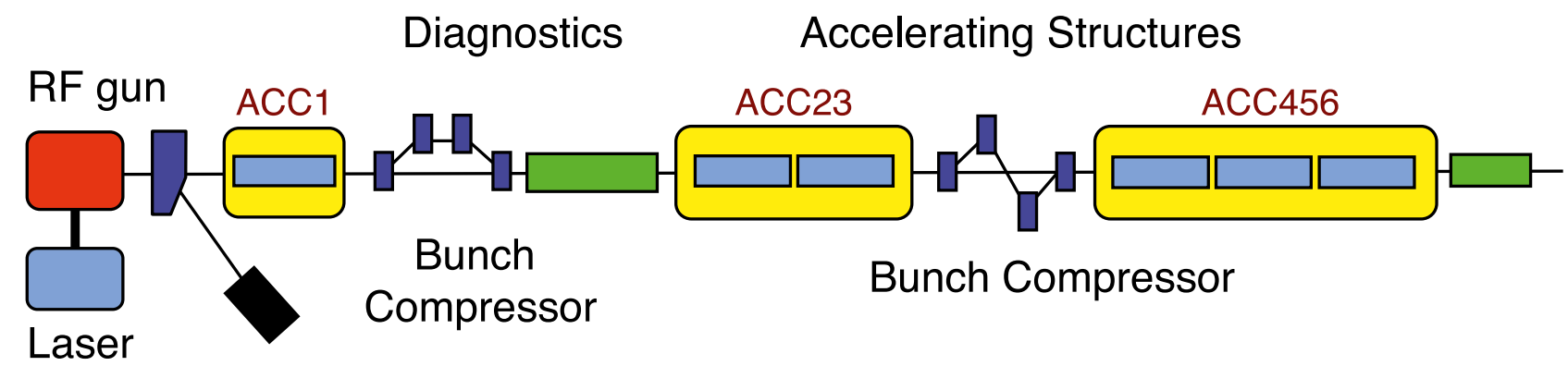

Figure 2.36 FLASH layout (2009).

Design beam parameters for FLASH are quite close to those of the ILC. Both are listed in Table 2.9 together with design parameters for the European XFEL and performance goals for the 9-mA experiment itself. Even so, achieving these beam parameters is a major challenge for FLASH, since the average power and pulse length are well beyond typical parameters for FEL user operation (less than 30 bunches per pulse and less than $1 \mathrm{nC}$ per bunch).

Extrapolation from the 9-mA experiment to the ILC involves not just beam parameters, but also operating gradients of the superconducting cavities. Gradient limits for the 48 FLASH cavities are shown in Figure 2.37. The gradient limits of the 24 cavities in ACC456 range from $23 \mathrm{MV} / \mathrm{m}$ to $32 \mathrm{MV} / \mathrm{m}$, with the first four cavities in ACC6 all having gradient limits above $30 \mathrm{MV} / \mathrm{m}$, and hence maximum operating gradients, are comparable to those planned for the ILC.

\begin{tabular}{|c|c|c|c|c|}
\hline Parameter & Design parameters & & & Goal \\
\hline & XFEL & ILC & FLASH & 9-mA experiment \\
\hline Bunch charge $(\mathrm{nC})$ & 1 & 3.2 & 1 & 3 \\
\hline Bunch repetition rate $(\mathrm{MHz})$ & 5 & 2.7 & 9 & 3 \\
\hline Number of bunches & 3,250 & 2,625 & 7,200 & 2,400 \\
\hline Pulse length (ms) & 650 & 970 & 800 & 800 \\
\hline Average current (mA) & 5 & 9 & 9 & 9 \\
\hline Average beam power $(\mathrm{kW})$ & & & 36 & 36 \\
\hline
\end{tabular}

Table 2.9 XFEL, ILC, and FLASH design parameters, and goals of the $9-\mathrm{mA}$ experiment. 


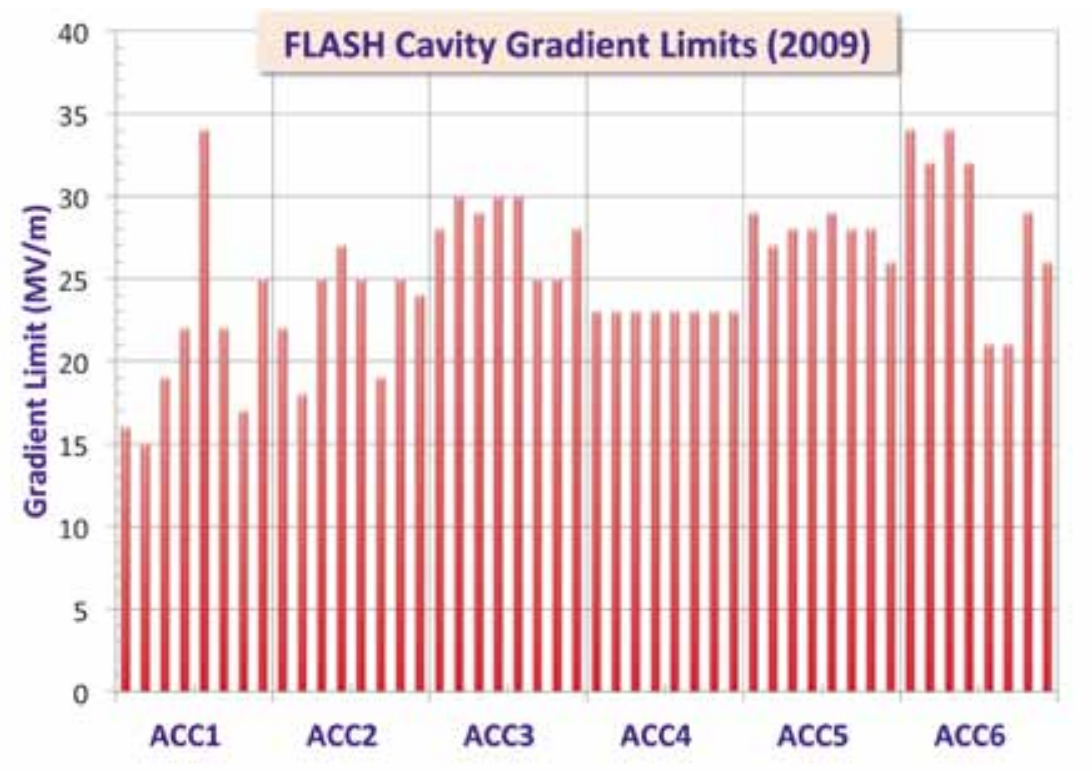

\section{Beam studies}

There have been two periods of dedicated 9-mA studies with high-power beams: 48 hours in September 2008 and a two-week period in September 2009.

In September 2008, the linac operated at a $1-\mathrm{MHz}$ bunch repetition rate with $3 \mathrm{nC}$ per bunch, and pulse lengths of 550 bunches were reached before the studies were cut short by a vacuum event in the final section of the beam transport line. This vacuum event prompted DESY to develop several new diagnostics for monitoring the beam position and monitoring beam spill. Beam position monitors using in-air magnetic loops and diamond/sapphire blades were installed immediately before the beam dump, and fourquadrant beam loss monitors using Cerenkov fibres and ion chambers were installed along the last several metres of the beam transport line. These new diagnostics were installed immediately prior to the two-week 9-mA study period in September 2009. It was during these two weeks of studies that the primary study goal for TD Phase 1 was achieved. Highlights of the studies results and achievements are described in the next section.

\section{Achievements during TD Phase 1}

A major milestone was reached during the September 2009 studies when high-power beam operation was demonstrated over a range of beam currents and pulse lengths. The highlighted progress is summarised in Table 2.10. 


\begin{tabular}{|c|c|c|}
\hline Metric & Goal & Achieved \\
\hline \multirow[t]{4}{*}{ Bunches per pulse } & $800 \times 3 \mathrm{nC}(1 \mathrm{MHz})$ & $800 \times 3 \mathrm{nC}$ \\
\hline & $2,400 \times 3 \mathrm{nC}(3 \mathrm{MHz})$ & $1,800 \times 3 \mathrm{nC}$ \\
\hline & & $2,100 \times 2.5 \mathrm{nC}$ \\
\hline & & $\sim 2,400 \times 2 \mathrm{nC}$ \\
\hline Charge per pulse & 7,200 nC@3 MHz & 5,400 nC@ 3 MHz \\
\hline \multirow[t]{2}{*}{ Beam power } & $36 \mathrm{~kW}$ & $22 \mathrm{~kW}$ \\
\hline & $(7,200 \mathrm{nC}, 5 \mathrm{~Hz}, 1 \mathrm{GeV})$ & $(5,400 \mathrm{nC}, 5 \mathrm{~Hz}, 800 \mathrm{MeV})$ \\
\hline
\end{tabular}

Table 2.10 Goals and results achieved in the FLASH

9-mA experiment.

In summary, the following machine parameters were achieved:

- 15 continuous hours of running with $3 \mathrm{~mA}$ and 800- $\mu$ s bunch trains

- Running at around $9 \mathrm{~mA}$ with bunch trains of 500 to $600 \mu \mathrm{s}$ for several hours

- Full pulse length (80o $\mu$ s, around 2,40o bunches) at around $6 \mathrm{~mA}$ for shorter periods

- Energy deviations within long bunch trains: less than $0.5 \%$ pulse-pulse at $7 \mathrm{~mA}$

- Energy jitter pulse-pulse with long bunch trains: around $0.13 \% \mathrm{rms}$ at $7 \mathrm{~mA}$

Reaching and operating the linac with high-power levels was unquestionably challenging, particularly with respect to minimising bunch-by-bunch fractional beam loss and with regard to exception handing. These, however, are operational issues, and are not indicative of any fundamental issues associated with operating a high-power superconducting linac. On the contrary, the test results achieved in September 2009 are considered by the GDE to be sufficient to have demonstrated the feasibility of a high-power superconducting electron linac. The requirement to minimise beam scraping plays into many different operational aspects, since beam scraping can occur from wrong steering, energy changes, beam size and beam stabilisation.

\section{Energy stability}

The beam energy must be stabilised over two timescales: long-term pulse-topulse stability over minutes and hours and energy deviations within a bunch train. Representative measurements of energy stability over both timescales are summarised above.

The main challenge for stabilising energy deviations within the bunch train is the compensation of transients from beam loading and Lorentz force detuning, which are a function of beam current and cavity gradient respectively. Both effects are largely repetitive from pulse to pulse: they can be compensated using 'iterative learning feed-forward' to progressively modify the RF power feed-forward drive waveforms to minimise the repetitive transients on the cavity field vector sum. Examples of energy deviations within long bunch trains for $3 \mathrm{~mA}$ and for $7.5 \mathrm{~mA}$ currents are shown in Figure 2.38. 
Figure 2.38 Achieved energy stability during bunch train at different currents.
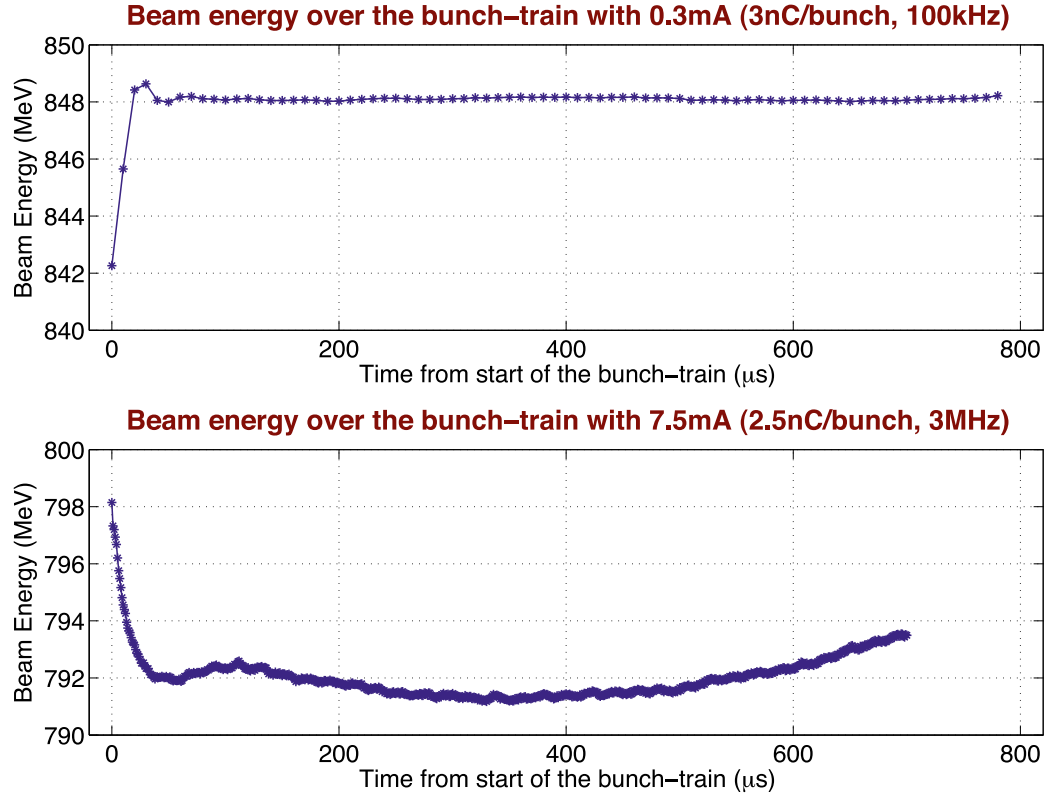

\section{Effects of beam loading}

The effect of beam loading on individual cavity gradients can be seen in Figure 2.39 for $3 \mathrm{~mA}$ and $7.5 \mathrm{~mA}$. Although the vector sums are flat, the individual cavities charge or discharge at a rate that depends on the beam current. These gradient 'tilts' can minimised by tailoring individual cavity forward power ratios and loaded Qs to the operating gradient and beam current. The extent to which this can be accomplished will be a subject of study during TD Phase 2. Examples of energy stability over the bunch train at $3 \mathrm{~mA}$ and $7.5 \mathrm{~mA}$ are shown in Figure 2.39.

Figure 2.39 Beam loading-induced gradient tilts at different currents.
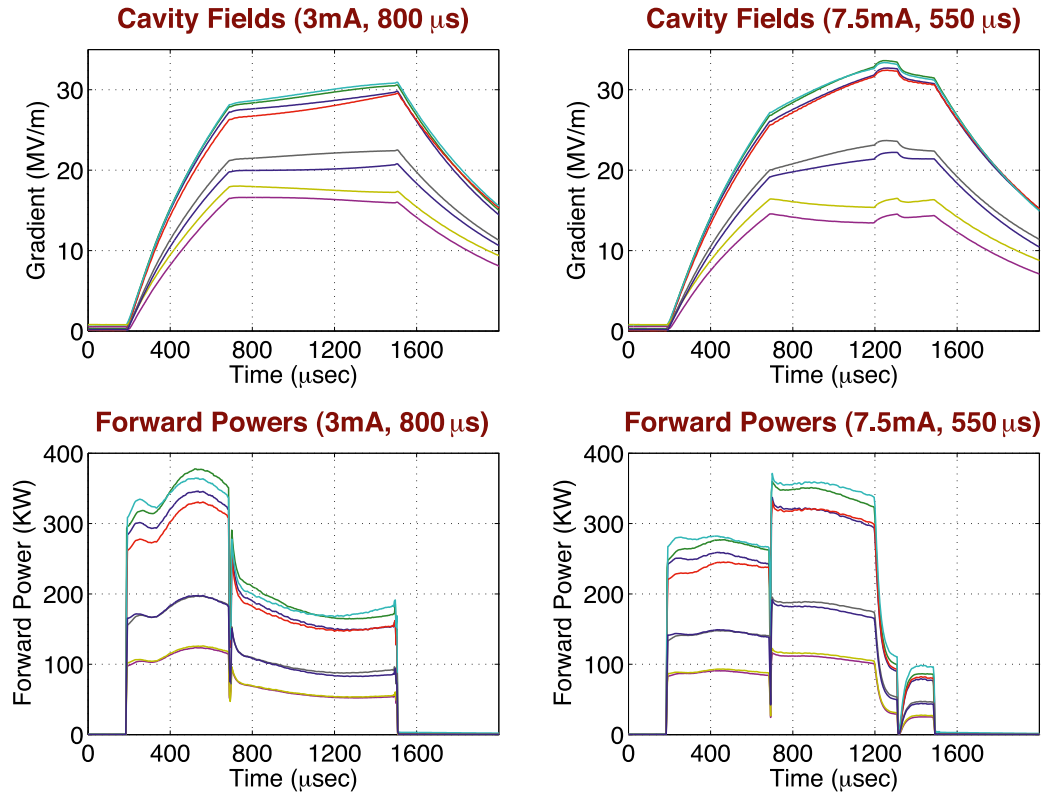


\section{Outlook}

Since September 2009, there have been several major upgrades to the FLASH accelerator to increase the reach and performance of FEL user operation. Upgrades included installation of a seventh cryomodule to raise the maximum energy to $1.25 \mathrm{GeV}$ and a third-harmonic cavity in the injector to linearise bunch compression and increase the peak bunch current. Significant upgrades have also been made to the low-level RF systems, with the introduction of fast beam-based feedback during the bunch trains, improvements in pulse-to-pulse learning feed-forward and beam loading compensation, and expansion of exception handling. The 9-mA studies will reap substantial benefit from these upgrades and the resulting performance improvements, such as the more than ten-fold reduction in rms energy jitter as shown in Figure 2.40 .
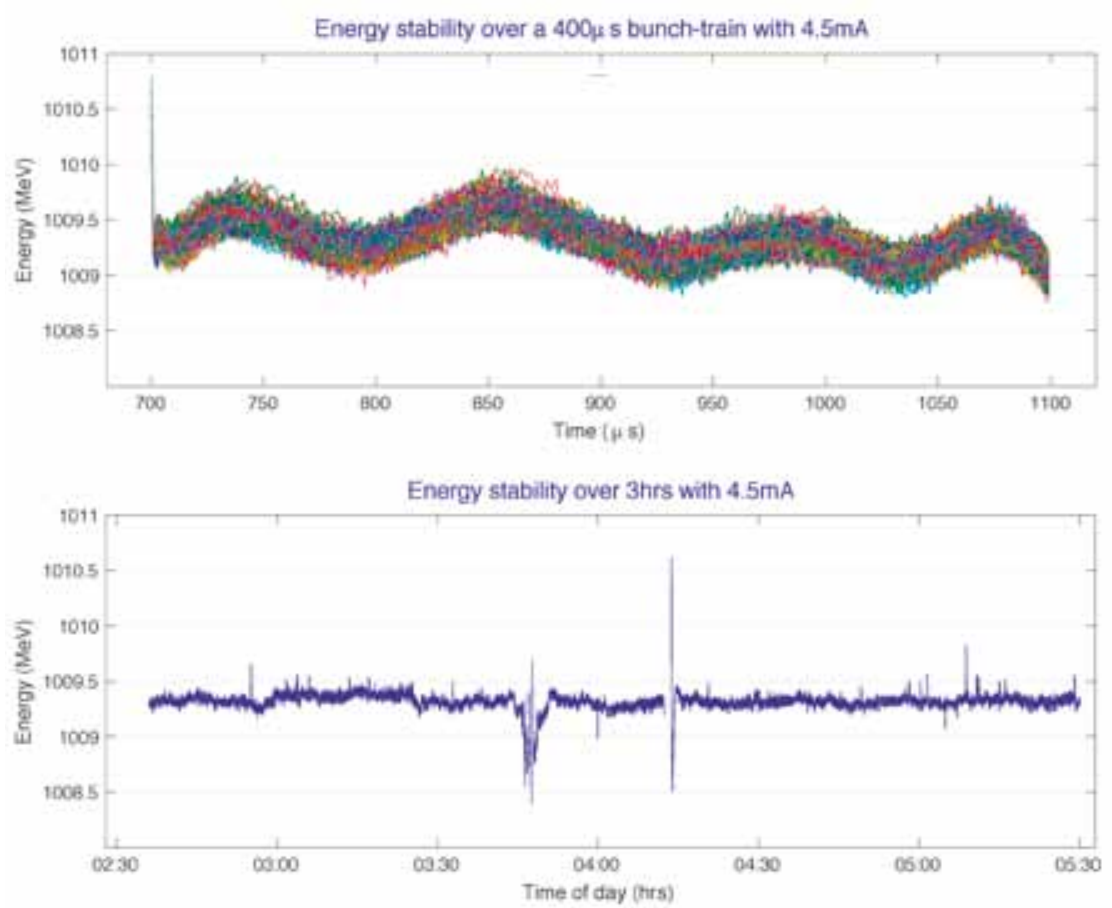

During TD Phase 2, 9-mA studies will shift emphasis from demonstrating feasibility of operations to studying 'operation at the limits,' such as required gradient margins and RF power overhead. Key issues will be tight control of beam-loading-induced gradient tilts, accurate compensation of Lorentz force detuning using fast piezo tuners, and tight control of beam loading-induced transients.

In summary, accomplishments during TD Phase 1 have demonstrated reliable operation of the FLASH linac with high power ILC-like beams, and hence the primary 9-mA experiment goals for TD Phase 1 have been achieved. Future 9-mA studies will be directed towards characterising operation with these high-power beams, and in particular, to assess the minimum gradient margin and RF power overhead required for reliable operation of the ILC. 


\section{SUPERCONDUCTING RADIOFREQUENCY TECHNOLOGY}

References

[2-1] ILC-GDE Technical Design Phase R\&D Plan (Rel.

5, 2010): http://ilcdoc.linearcollider.org/record/15442

[2-2] A. Yamamoto for the ILC-GDE SCRF

collaboration, "Global R\&D effort for the ILC SCRF

LINAC Technology," Proc. EPAC-08, Genova, (2008).

[2-3] A. Yamamoto for the ILC-GDE SCRF

collaboration, "Superconducting RF cavity development

for the International Linear Collider," IEEE Trans. Appl.

Supercond. 19 No. 3, p. 138 (2009).

[2-4] ITRP report: (2004). http://www.fnal.gov/

directorate/icfa/ITRP_Report_Final.pdf

[2-5] TESLA TDR edited by F. Richard et al. (2001):

http://flash.desy.de/tesla/tesla_documentation/\#e1509

[2-6] J. Wiener et al., TTC Report 2008-2 (2008).

[2-7] G. Ciovati et al., TTC Report 2008-5 (2008):

http://flash.desy.de/reports_publications/tesla_reports/

tesla_reports_2008/

[2-8] A. Yamamoto, presented at the1st Baseline

Assessment Workshop (BAW1): http://ilcagenda.

linearcollider.org/conferenceOtherViews.

py?view=standard\&confld=4593

[2-9] H. Hayano, "Asian Region Laboratory Plans,"

presented at the Superconducting RF Cavity

Technology and Industrialization Workshop (A Satellite

Workshop at IPAC-10, Kyoto, 2010):

http://ilcagenda.linearcollider.org/conferenceDisplay.

py?confld $=4530$

[2-10] K. Ueno et al., "Development of electro-polishing

facility in KEK," Proc. of 5th Japanese Accelerator

Society meeting (2008).

[2-11] Y. Yamamoto et al., "Summary of results and

development of online monitor for T-mapping/X-ray-

mapping in KEK-STF," Proc. IPAC-10, Kyoto (2010).

[2-12] Y. Iwashita et al., Phs. Rev. ST Accel. Beam, 11, 093501 (2008).

[2-13] K. Watanabe et al., "Repair techniques of superconducting cavity for improvement cavity performance at KEK-STF," Proc. IPAC-10, Kyoto. [2-14] R. Kephart, "American Region Laboratory Plans," presented at the Superconducting RF Cavity Technology and Industrialization Workshop (A Satellite Workshop at IPAC-10, Kyoto, 2010):

http://ilcagenda.linearcollider.org/conferenceDisplay. py?confld $=4530$

[2-15] J. Ozelis, "Initial Results from Fermilab's Vertical Test Stand for SCRF Cavities," Proc. 13th International Workshop on RF Superconductivity (SRF2007), Beijing (2007).
[2-16] A. Hocker et al., "Commissioning and early operating experience with the Fermilab horizontal test facility," Proc. 13th International Workshop on RF Superconductivity (SRF2007), Beijing (2007). [2-17] R. Carcagno et al., "Control System Design for Automatic Cavity Tuning Machines," Proc. 2009 Particle Accelerator Conference, Vancouver (2009). [2-18] Jan-Hendrik Thie et al., "Mechanical Design of Automatic Cavity Tuning Machines," Proc. 14th International Workshop on RF Superconductivity (SRF2009), Berlin (2009).

[2-19] D. Reschke et al., "Preparatory Procedure and Equipment for the European $X$-ray Free Electron Laser Cavity Implementation," Phys. Rev. ST Accel. Beams 13, 071001 (2010).

[2-2O] T. Arkan et al., "Superconducting RF Cryomodule Production and Testing at Fermilab," Proc. XXV Linear Accelerator Conference (LINAC10), Tsukuba, Japan, 2010.

[2-21] C.M. Ginsburg, M.P. Kelly et al.,

"Superconducting RF Cavity Production Processing

and Testing at Fermilab," Proc. XXV Linear Accelerator Conference (LINAC10), Tsukuba (2010).

[2-22] A. Rowe, "ANL/Fermilab SCPF EP/HPR

Processing Status and Plans," Linear Collider Forum of the Americas, (2009).

[2-23] M. Kelly et al., "Surface Processing Facilities

for Superconducting RF Cavities at ANL," LINAC-08 (2008).

[2-24] 0. Napoli, "E-XFEL cryomodule production plan and model," presented at the Superconducting RF

Cavity Technology and Industrialization Workshop (A

Satellite Workshop at IPAC-10, Kyoto, 2010):

http://ilcagenda.linearcollider.org/conferenceDisplay. py?confld $=4530$

[2-25] L. Lilje, "R\&D in RF superconductivity to support the International Linear Collider," Proc. PAC-08,

Albuquerque, p. 2559 (2007).

[2-26] See an appendix of the TTC Report 2008-05,

"ILC R\&D Board Task Force on high gradient (S0/S1)," (2006).

[2-27] R. Geng "Long-term cavity R\&D overview," presented at Int. Workshop on Linear Collider 2010, IWLC2010, Acc-WG3, 10-10-21, Geneve (2010).

[2-28] See ILC Newsline, http://www.linearcollider.org/ newsline/readmore_20091029_ftr1.html

[2-29] C. Ginsburg and A. Yamamoto presented at Int. Workshop on Linear Collider 2010, IWLC2010, Acc-WG3, 21 October 2010: http:// ilcagenda.linearcollider.org/conferenceOtherViews. py?view=standard\&confld $=4507$
[2-30] K. Saito et al., "Gradient yield improvement efforts for single and multi-cells and progress for very high gradient cavities," SCRF-07, Beijing, TU202 (2007).

[2-31] R. Geng, "Review for new shapes for high gradients," Physica C 441 p. 145 (2006).

[2-32] N. Ohuchi, et al, LINAC10, Tsukuba, September 2010, M0302, http://jacow.org

[2-33] N. Ohuchi, et al., EPAC08, Genoa, June 2008, MOPP144, p. 892 (2008), http://jacow.org

[2-34] http://www.xfel.eu

[2-35] N. Phinney, et al., ILC RDR Accel., ILC-Report-2007-001, p.III-171, (2007): http:// ilcdoc.linearcollider.org/record/6321/files/ILC_RDR_ Volume_3-Accelerator.pdf?version $=4$

[2-36] C. Pagani, et al., "TESLA Cryogenic Accelerator Modules," TESLA Report 2001-36.

[2-37] T. Peterson, "Cryomodule Thermal Intercept Optimization Strategy," TILC08-Sendai, March 3-6, Sendai, 2008: http://ilcagenda. linearcollider.org/conference0therViews. py?view=standard\&confld $=3154$

[2-38] Reference Design Report, Accelerator, ILC-Report-2007-001, 2007: http://lcdev.kek.jp/RDR/ RDRall.pdf

[2-39] Christopher Nantista and Chris Adolphsen, "Klystron Cluster Scheme for ILC High Power RF Distribution," presented at the 2009 Particle Accelerator Conference (PAC09), Vancouver (2009); SLAC-PUB-13696.

[2-40] C. Burkhart, et al., "ILC Marx Modulator Development Program Status," presented at the 1st International Particle Accelerator Conference (IPAC10), Kyoto, (2010).

[2-41] M.A. Kemp, et al., "Status Update on the Second-Generation ILC Marx Modulator Prototype," IEEE Power Modulator Conference, Atlanta, (2010).

[2-42] Chris Adolphsen, "ILC RF System R\&D," presented at the 1st International Particle Accelerator Conference (IPAC10), Kyoto, Japan, May 23-28, 2010. [2-43] Christopher D. Nantista and Chris Adolphsen, "A Variable Directional Coupler for an Alternate ILC High-Power RF Distribution Scheme," presented at the 2006 Linear Accel. Conf. (LINAC06), Knoxville,(2006); SLAC-PUB-12372.

[2-44] Christopher Nantista et al., "Progress in L-Band Power Distribution System R\&D at SLAC," presented at the XXIV Linear Accelerator Conference (LINAC08), Victoria, (2008); SLAC-PUB-13438. 
[2-45] S. Fukuda, "DRFS design and R\&D

status, and Installation strategy," presented

at the 1st Baseline Assessment Workshop

(BAW-1), KEK, Tsukuba (2010): http://ilcagenda.

linearcollider.org/conferenceOtherViews.

py?view=standard\& confld $=4593$

[2-46] S. Fukuda, "DRFS test preparation for

S1-Global," presented at Int. Workshop on Linear

Collider, IWLC2010, Geneva, (2010): http://

ilcagenda.linearcollider.org/conferenceOtherViews.

py?view=standard\&confld $=4507$

[2-47] A. Yamamoto, "ILC SCRF Update," presented at the Int. Workshop on Linear Collider, Geneva, (2010):

http://ilcagenda.linearcollider.org/

conference0therViews. py?view $=$ standard $\&$ confld $=4507$

[2-48] N. Walker, et al, "Operation of the FLASH Linac with Long Bunch Trains and High Average Current,"

(PAC 2009): http://accelconf.web.cern.ch/AccelConf/

PAC2009/papers/we6pfp109.pdf

[2-49] R. Kephart, "American Region Laboratory

Plans," presented at the Superconducting RF Cavity

Technology and Industrialization Workshop (A Satellite

Workshop at IPAC-10, Kyoto, 2010): http://ilcagenda.

linearcollider.org/conferenceDisplay.py?confld=4530

[2-50] H. Hayano, "Asian Region Laboratory

Plans," presented at the Superconducting RF Cavity

Technology and Industrialization Workshop (A Satellite

Workshop at IPAC-10, Kyoto, 2010): http://ilcagenda.

linearcollider.org/conferenceDisplay.py?confld=4530.

[2-51] STF group, "STF Phase-1 Activity Report," KEK

Report 2009-3, April 2009:

[2-52] N. Ohuchi, et al, "S1-Global collaborative efforts

8-cavity-cryomodule: 2 Fermilab, 2 DESY and 4 KEK,"

Proceedings of LINAC 10, Tsukuba, Japan, 2010. 


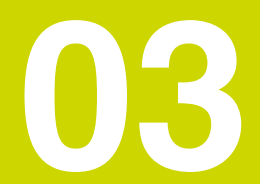

3.1 THE ELECTRON CLOUD R\&D PROGRAMME AT CESRTA AND OTHER LABORATORIES

3.2 THE ATF2 FINAL FOCUS TEST BEAMLINE AT KEK

3.3 ACCELERATOR SYSTEMS R\&D

\section{ACCELERATOR SYSTEM R\&D}




\subsubsection{Introduction}

One of the principal R\&D issues for the positron damping ring of the ILC is to ensure that the build-up of the electron cloud in the vacuum chambers can be kept below the levels at which electron cloud-induced emittance growth and beam instabilities occur. During Phase I (2008-2010) of the ILC Technical Design Phase (TDP) a focused effort to study methods of suppressing the electron cloud as well as measuring its impact on ultra-low emittance beams was undertaken at the Cornell Electron-Positron Storage Ring Test Accelerator (CesrTA). In addition, work has also been underway at various laboratories around the world to develop better techniques to mitigate the build-up of the electron cloud. Section 3.1.2 describes the research effort being carried out at Cornell University with the CesrTA collaboration [3-1, 2], while section 3.1.3 describes the work that is in progress at various other laboratories around the world. As part of this coordinated global programme, a major emphasis has been placed on developing and benchmarking simulation tools as well as measurement techniques. In October 2010, the ECLOUD10 Workshop was held at Cornell University [3-3]. The workshop presentations provide a comprehensive overview of the recent activities.

In order to incorporate the research results into the ILC damping ring design, an ECLOUD Working Group has been formed whose main objective is to provide recommendations on the electron cloud mitigation techniques to apply to the damping ring design based on the results of the R\&D programme $[3-4,5]$. This objective has recently been achieved in a dedicated Working Group meeting [3-6] during the ECLOUD1o Workshop, with a significant level of participation by the experts attending the workshop. The preliminary recommendations are summarised in section 3.1.4.
3.1 THE ELECTRON CLOUD R\&D PROGRAMME AT CESRTA AND OTHER LABORATORIES

\subsubsection{The CesrTA R\&D programme}

The CesrTA research programme was approved in late 2007 to carry out electron cloud R\&D in support of the ILC technical design. The first dedicated experiments using the Cornell Electron-Positron Storage Ring (CESR) began in March 2008 at the conclusion of 28 years of colliding beam operations for the CLEO experiment [3-7]. Two principal goals were specified for the programme. The first was to characterise the build-up of the electron cloud in each of the key magnetic field regions of the accelerator, particularly in the dipoles and wigglers, and to study the most effective methods of suppressing it in each of these regions. This required the design and installation of detectors to study the local build-up of the cloud in each of these environments as well as a supporting simulation programme to fully characterise and understand the results. The second goal was to study the impact of the electron cloud on ultra-low emittance beams. The ILC damping ring design targets a geometric vertical emittance of 2 picometre radians (pm.rad); no positron ring has been operated in this emittance regime. By benchmarking electron cloud instability and emittance growth simulations in a regime closer to that specified for the damping ring, confidence in projections of the final damping ring performance can be significantly improved. This in turn will determine whether further R\&D is required to achieve the necessary design specifications. 
Table 3.1 2-GeV and 5-GeV lattice parameters for CesrTA.
In order to carry out these measurements, CESR had to be reconfigured as a damping ring and upgraded with the necessary beam instrumentation for low-emittance optics correction and characterisation of the resulting beams.

\section{Conversion of CESR to a damping ring test accelerator configuration}

Modification of CESR into a damping ring configuration involved three main thrusts:

1. Relocation of six of the twelve CESR-c damping wigglers $[3-8,9]$ to the Lo straight section to enable ultra-low emittance CesrTA operation [3-7].

2. Upgraded beam instrumentation to achieve and characterise ultra-low emittance beams. This included deployment of a high-resolution beam position monitoring system [3-10] and X-ray beam-size monitors for both positron and electron beams [3-11].

3. Addition of vacuum system diagnostics for characterisation of local electron cloud growth in a range of vacuum chambers. Local electron cloud diagnostics include retarding field analysers [3-12, 13], transverseelectric-wave transmission hardware [3-14] and shielded pickups for time-resolved measurements [3-15]

Table 3.1 shows the CesrTA lattice parameters for operation at 2 and 5 gigaelectronvolts $(\mathrm{GeV})$. At $2 \mathrm{GeV}, 90 \%$ of the synchrotron radiation power is provided by the twelve damping wigglers and a horizontal emittance of 2.6 nanometre radians ( $\mathrm{nm} \cdot \mathrm{rad}$ ) is obtained [3-16]. During Phase I of the CesrTA programme, a vertical emittance target of less than $20 \mathrm{pm} \cdot \mathrm{rad}$ (ten times the ILC damping ring vertical emittance target) was specified. A key element of the R\&D programme is the flexibility of CESR operation. CESR allows operation between 1.8 and $5.3 \mathrm{GeV}$ with both positron and electron beams. The ability to operate over a wide range of energies, bunch spacings and bunch intensities enables systematic studies of primary photoelectron and secondary electron contributions to electron cloud build-up in the vacuum chambers, which are not feasible at any other facility.

\begin{tabular}{|c|c|c|c|c|c|}
\hline \multirow{13}{*}{$\begin{array}{l}\text { Table 3.1 2-GeV and 5-GeV lattice parameters } \\
\text { for CesrTA. }\end{array}$} & \multirow{2}{*}{$\begin{array}{l}\text { Energy } \\
\text { Number of wigglers }\end{array}$} & & \multirow[t]{2}{*}{$\mathrm{GeV}$} & \multirow{2}{*}{$\begin{array}{l}2.085 \\
12\end{array}$} & \multirow{2}{*}{$\begin{array}{l}5.0 \\
6\end{array}$} \\
\hline & & & & & \\
\hline & Wiggler field & & $\mathrm{T}$ & 1.9 & 1.9 \\
\hline & Horizontal tune & $Q_{x}$ & & 14.57 & \\
\hline & Vertical tune & $Q_{y}$ & & 9.6 & \\
\hline & Longitudinal tune & $\mathrm{Q}_{2}$ & & 0.075 & 0.043 \\
\hline & RF voltage & $\mathrm{V}_{\mathrm{RF}}$ & MV & 8.1 & 8 \\
\hline & Horizontal emittance & $\varepsilon_{\mathrm{x}}$ & $\mathrm{nm} \cdot \mathrm{rad}$ & 2.6 & 35 \\
\hline & Damping time constant & $\tau_{x, y}$ & $\mathrm{~ms}$ & 57 & 20 \\
\hline & Momentum compaction & $a_{p}$ & & $6.76 \times 10^{-3}$ & $6.23 \times 10^{-3}$ \\
\hline & Bunch length & $\sigma_{1}$ & $\mathrm{~mm}$ & 9.2 & 15.6 \\
\hline & Relative energy spread & $\sigma_{\mathrm{E}} / \mathrm{E}$ & & $0.81 \%$ & $0.93 \%$ \\
\hline & Bunch spacing & $t_{b}$ & ns & $\geq 4$, steps of 2 & \\
\hline
\end{tabular}


A novel element of the CesrTA upgrade has been the development of a highresolution X-ray beam size monitor capable of single-pass measurements of each bunch in a train. Figure 3.1 shows one of the indium-gallium-arsenide detectors wire-bonded to its circuit board along with a single-pass fit of data acquired using pinhole imaging with a 1-milliamp $(\mathrm{mA})$ bunch. In addition to pinhole imaging, coded aperture and Fresnel zone plate optics have also been installed in both the positron and electron beam lines. These detectors are our principal tools for verifying the vertical beam size in the ultra-low emittance machine optics.
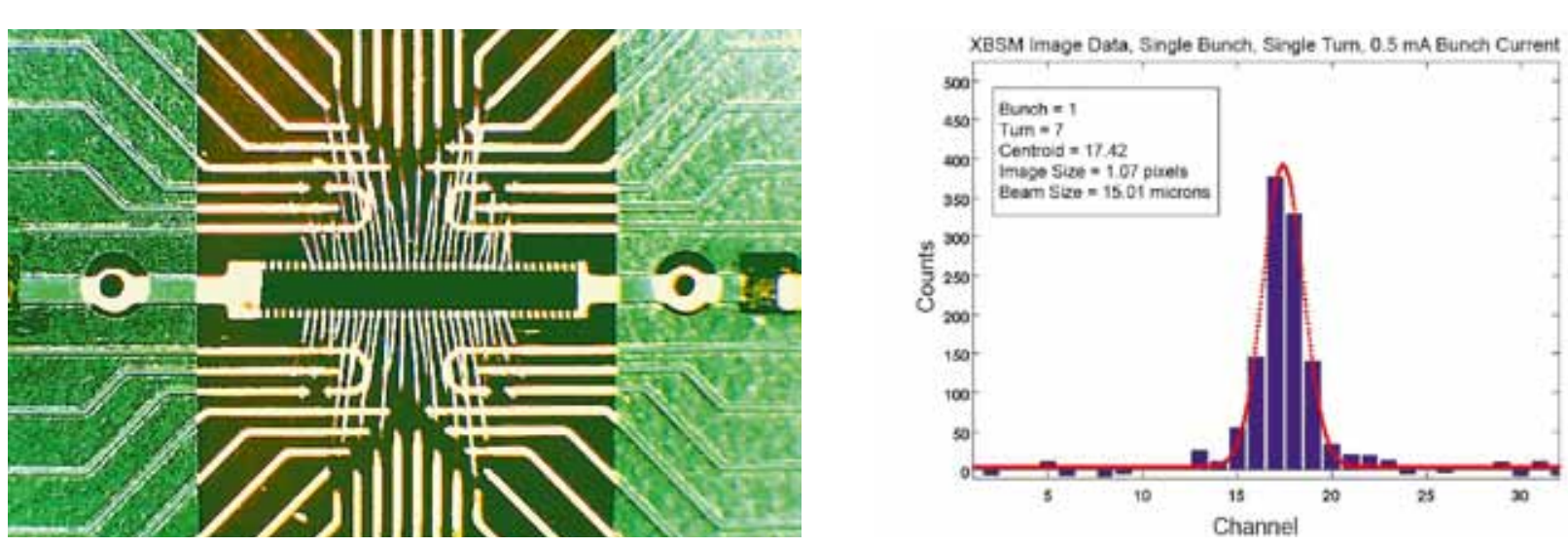

Figure 3.1 Left: an X-ray beam size monitor detector, an indium-gallium-arsenide diode array, mounted on its circuit board. 32 diodes of 400-micrometre width and 50-micrometre pitch are utilised in each detector. Right: a single-turn fit to data acquired from a bunch with $0.8 \times 10^{10}$ particles (at 2.1-GeV beam energy) using a heavy-metal slit as the $\mathrm{X}$-ray imaging optic.

Figure 3.2 shows the layout of the Lo straight section after installation of the wiggler string. This region is one of four dedicated CesrTA electron cloud experimental areas. It is equipped with extensive diagnostics to study the growth and mitigation of the electron cloud in wigglers. A second electron cloud experimental region was installed on the opposite side of CESR in the L3 straight section.



Figure 3.2 Layout of the CESR LO wiggler straight and electron cloud experimental region with a cutaway view of the CLEO detector. Six superconducting CESR-c type wigglers are deployed in the straight, which is configured for zero dispersion operation. The straight section includes extensive vacuum diagnostics: retarding field analysers, residual gas analyser, and transverse electric wave measurement hardware. 
Figure 3.3 Layout of the CESR L3 straight and electron cloud experimental region. Tests of electron cloud mitigations in drift, dipole and quadrupole chambers are possible in this region. Additionally, an in situ secondary-electron-yield station is also installed, which allows characterisation of the rate of processing and equilibrium secondary electron yield properties of various vacuum system technical surfaces.
Figure 3.3 shows the layout of the L3 region. It supports four electron cloud experiments: a large bore quadrupole housing a test chamber; the Positron Electron Project (PEP) II chicane for dipole chamber tests, which was relocated from SLAC after the early termination of PEP II operations; a drift chamber test section currently configured for testing titanium-zirconium-vanadium (TiZrV) (NEG) test chambers; and an in situ secondary-electron yield measurement station, which supports studies of the processing rates and equilibrium secondary electron yield properties of various technical surfaces. In addition to the Lo and L3 experimental regions, two arc sections were configured for flexible installation of experimental drift chambers to study the performance of various mitigations in the photon environment of the CESR arcs.



\section{Electron cloud build-up and mitigation studies}

Retarding field analysers deployed at approximately 30 locations around CESR have enabled the detailed study of local cloud build-up in variety of vacuum chambers under a range of experimental conditions [3-17, 18]. The analysers provide a time-averaged current readout at each location. The majority of deployed retarding field analysers utilise a segmented design to provide geometric information about the cloud build-up around the azimuth of the vacuum chamber. Analyser data taken in vacuum chambers fabricated with cloud mitigations provides the foundation for comparison of the efficacy of different electron cloud mitigation methods. An active effort is underway to model this analyser data in order to determine the secondary-electron yield and photoelectron yield parameters of the vacuum chambers treated with mitigations $[3-18,19,20]$. In addition to the retarding field analyser studies, transverse-electric-wave transmission methods [3-21] are also being used to characterise the build-up around the ring and a significant simulation effort is underway to take full advantage of these results [3-22, 23, 24]. A final method to study local cloud build-up is shielded pickup measurements [3-25], which are providing additional constraints on the vacuum chamber surface parameters for the chambers in which they are installed. 
Table 3.2 summarises the range of chamber surfaces and mitigation methods that were prepared for testing during Phase I of the CesrTA R\&D programme. Figure 3.4 shows a comparison of the performance of various chamber surfaces in a dipole field along with a plot of the evolution of the transverse distribution of the electron cloud that develops in the dipole chamber as a function beam current. While coating with a low secondary electron yield material such as titanium nitride significantly reduces the growth of the cloud in this environment, the use of a grooved surface with titanium nitride coating is clearly superior.

\begin{tabular}{|c|c|c|c|c|c|c|}
\hline Mitigation & Drift & Quadrupole & Dipole & Wiggler & $\begin{array}{l}\text { Institutions providing } \\
\text { chambers }\end{array}$ & $\begin{array}{l}\text { Table 3.2 Vacuum chambers fabricated for testing } \\
\text { during Phase I of the CesrTA R\&D programme. }\end{array}$ \\
\hline $\mathrm{Al}$ & $\sqrt{ }$ & $\sqrt{ }$ & $\vec{v}$ & & CU, SLAC & Checks indicate chambers for which data has \\
\hline $\mathrm{Cu}$ & $\bar{v}$ & & & $\bar{v}$ & CU, KEK, LBNL, SLAC & already been acquired. Dated entries indicate \\
\hline TiN on Al & $\sqrt{ }$ & $\sqrt{ }$ & $\checkmark$ & & CU, SLAC & $\begin{array}{l}\text { scheduled installation times for chambers yet to be } \\
\text { tested. CU stands for Cornell University }\end{array}$ \\
\hline TiN on $\mathrm{Cu}$ & v & & & $\sqrt{ }$ & CU, KEK, LBNL, SLAC & \\
\hline Amorphous $\mathrm{C}$ on $\mathrm{Al}$ & $\sqrt{ }$ & & & & CERN, CU & \\
\hline Diamond-like C on Al & $1 / 2011$ & & & & CU, KEK & \\
\hline NEG on SS & v & & & & $\mathrm{CU}$ & \\
\hline Solenoid windings & $\bar{v}$ & & & & $\mathrm{CU}$ & \\
\hline Fins with TiN on Al & v & & & & SLAC & \\
\hline $\begin{array}{l}\text { Triangular grooves } \\
\text { on } \mathrm{Cu}\end{array}$ & & & & $\bar{v}$ & CU, KEK, LBNL, SLAC & \\
\hline $\begin{array}{l}\text { Triangular grooves } \\
\text { with TiN on Al }\end{array}$ & & & $\sqrt{ }$ & & CU, SLAC & \\
\hline $\begin{array}{l}\text { Triangular grooves } \\
\text { with TiN on } \mathrm{Cu}\end{array}$ & & & & $1 / 2011$ & CU, KEK, LBNL, SLAC & \\
\hline Clearing electrode & & & & $\sqrt{ }$ & CU, KEK, LBNL, SLAC & \\
\hline
\end{tabular}
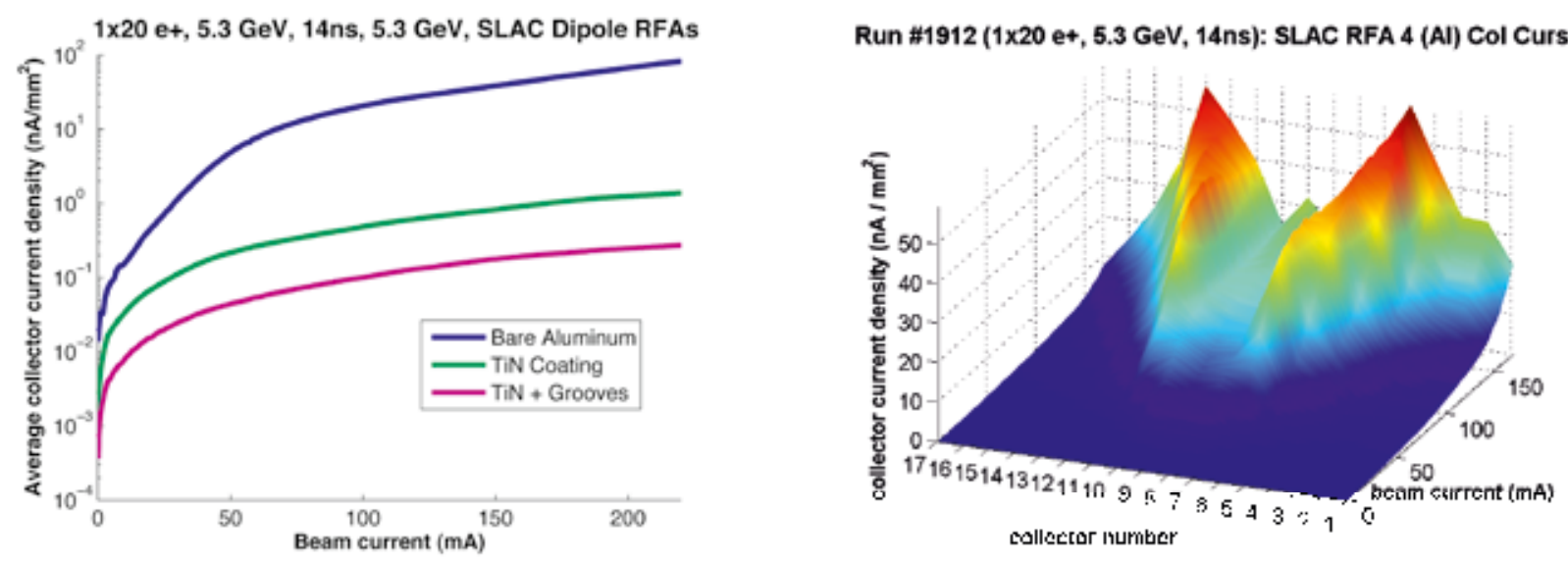

Figure 3.4 Left: the measured retarding field analyser current in a dipole versus beam current with a 20-bunch positron train for a bare aluminium surface, titanium nitride-coated surface and a grooved surface with titanium nitride coating. The efficacy of the grooved surface for suppressing the electron cloud is clearly evident. Right: the transverse shape of the electron cloud signal in the dipole retarding field analyser (aluminium chamber surface) as a function of beam current. 
Figure 3.5 Left: a grooved copper insert with $21.8^{\circ}$ triangular grooves having 1 -mm pitch for testing in a CesrTA wiggler. Right: a thin clearing electrode applied with a thermal spray method to the bottom half of another CesrTA experimental wiggler chamber.
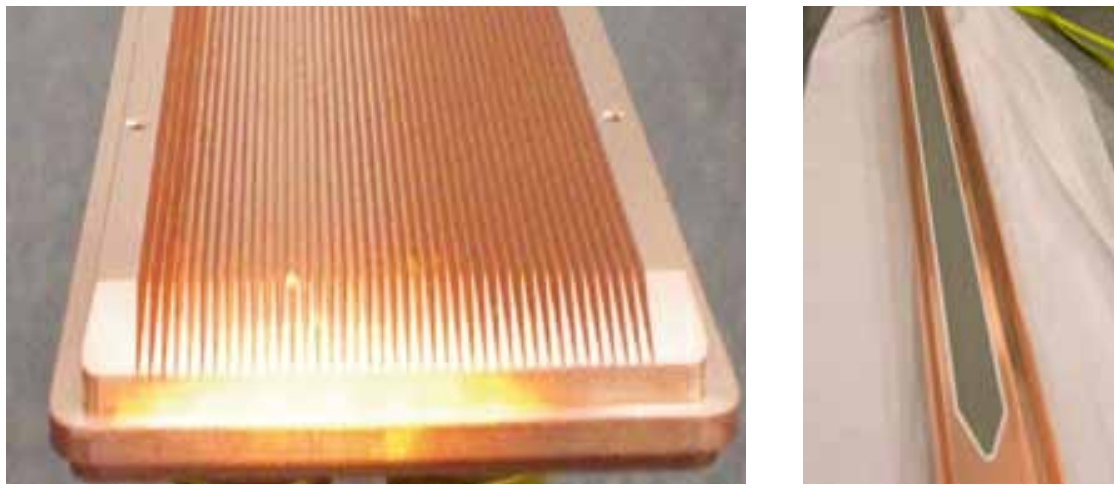

Figure 3.5 shows two of the mitigation methods that have been tested in the CesrTA high-field damping wigglers: triangular grooves and a clearing electrode. The clearing electrode is a very thin structure developed at KEK [3-26] that offers very good thermal contact with the vacuum chamber and minimal impact on the chamber aperture (see also section 3.1.3). A bare copper surface and a titanium nitride-coated copper surface have also been tested. The left plot in Figure 3.6 shows a comparison of the electron cloud growth as a function of beam current with each of these surfaces. The data indicate that the best cloud suppression in the wiggler region is obtained with the clearing electrode. One additional comparison remains: the testing of a grooved surface with titanium nitride coating. This test chamber has recently been installed in CESR and tests will take place over the next few months.

The right plot in Figure 3.6 shows the transverse distribution of the cloud present in the vertical field region of the wiggler (copper surface) as a function of the retarding grid voltage, which probes the energy spectrum of the electron cloud.
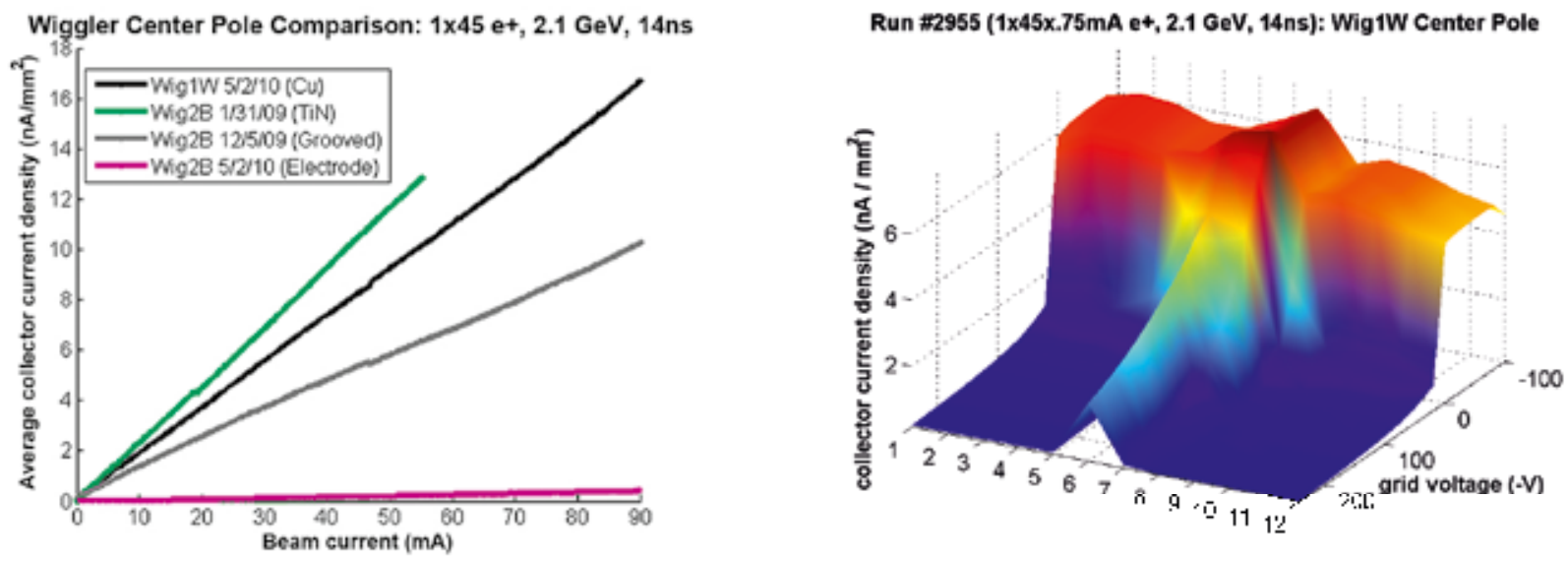

Figure 3.6 Left: the measured retarding field analyser current in a wiggler versus beam current with a 20-bunch positron train for a bare copper surface, a titanium nitridecoated copper surface, a grooved copper surface and a clearing electrode. The efficacy of the clearing electrode for suppressing the electron cloud is clearly evident. Right: the transverse shape of the electron cloud signal in the wiggler retarding field analyser as a function of retarding voltage. 
Studies of the electron cloud build-up in drift and quadrupole regions have also yielded important results. Drift measurements have been used to compare the performance of various coatings. A new coating of significant interest is amorphous carbon coating developed at CERN [3-27] for use in the Super Proton Synchrotron (SPS). Tests at CesrTA have afforded the opportunity to study the performance of this coating in the presence of synchrotron radiation. Initial studies show that the electron cloud mitigation performance of amorphous carbon is quite comparable to that of titanium nitride and that its vacuum performance is quite reasonable in an environment with significant photon flux. Continued testing will provide information about the long-term durability of this very promising coating. Vacuum chambers in quadrupole magnetic fields can show quite significant cloud build-up. Concerns about long-term trapping of the cloud in quadrupole fields [3-28] requires that cloud mitigation be incorporated into the ILC damping ring quadrupole vacuum chambers. Tests in CesrTA have demonstrated the effectiveness of titanium nitride coating in this region.

Low-emittance programme and studies of electron cloud-induced beam dynamics with low-emittance beams

The CesrTA low-emittance tuning effort provides the basis for studying the emittance-diluting effects of the electron cloud in a regime approaching that of the ILC damping rings. As of early 2010, the low-emittance tuning programme had resulted in reliable operation at or below the CesrTA Phase I vertical emittance of $20 \mathrm{pm} \cdot \mathrm{rad}$ [3-29] for both single- and multi-bunch operation as confirmed by X-ray beam size monitor measurements of the vertical beam size [3-30]. As of the end of 2010, vertical emittances less than $10 \mathrm{pm} \cdot \mathrm{rad}$ have been achieved.

A number of beam dynamics studies have been conducted in order to fully characterise the impact of the electron cloud on beams in CESR. As the electron cloud builds up along a bunch train, the focusing effect of the cloud on the beam causes the natural frequency of oscillation of each bunch (i.e. the horizontal and vertical betatron tunes) to shift with respect to the preceding bunch. Measurements of this electron cloud-induced coherent tune shift [3-31,32] for trains of electron and positron bunches, as well as for witness bunches at various positions behind a leading train, have provided an important probe of the integrated effect of the cloud around the ring. Systematic measurements over a wide range of beam conditions (varying beam energy, emittance, bunch current, bunch spacing and train lengths) are being used to validate our electron cloud models more thoroughly and have led to improved simulations, for example, for the ring photon propagation model [3-33], which are now being applied to the ILC damping ring.

A principal deliverable of the CesrTA programme is the characterisation of instability thresholds and emittance-diluting effects in the regime of ultra-low vertical emittance [3-34, 35, 36]. Figure 3.7 shows the observed beam motion spectrum for each bunch along a train obtained in these conditions. As described in the preceding paragraph, the development of the horizontal and vertical tune lines, denoted by $\mathrm{F}_{\mathrm{h}}$ and $\mathrm{F}_{\mathrm{v}}$, along the bunch train provides information about the electron cloud density experienced by each bunch. For a positron train, the attractive force of the bunch pinches the cloud into the bunch and can lead to the development of an oscillation of the bunch tail with respect to the head. This head-tail instability is expected to induce 
characteristic sidebands in the bunch motion spectrum. In Figure 3.7, the onset of the spectral lines denoted by $\mathrm{F}_{\mathrm{v}} \pm \mathrm{F}_{\mathrm{s}}$ part way along the bunch train indicate where the cloud density build-up has become sufficient for the onset of the instability.

Figure 3.7 Bunch-by-bunch power spectrum for a positron train with a nominal bunch current of 0.75 $m A /$ bunch. The horizontal $\left(F_{h}\right)$ and vertical $\left(F_{v}\right)$ tunes are clearly visible for all bunches. The onset of the sidebands labelled as $\mathrm{F}_{\mathrm{v}} \pm \mathrm{F}_{\mathrm{s}}$ are consistent with the onset of a head-tail instability around bunch number 15 in the train.

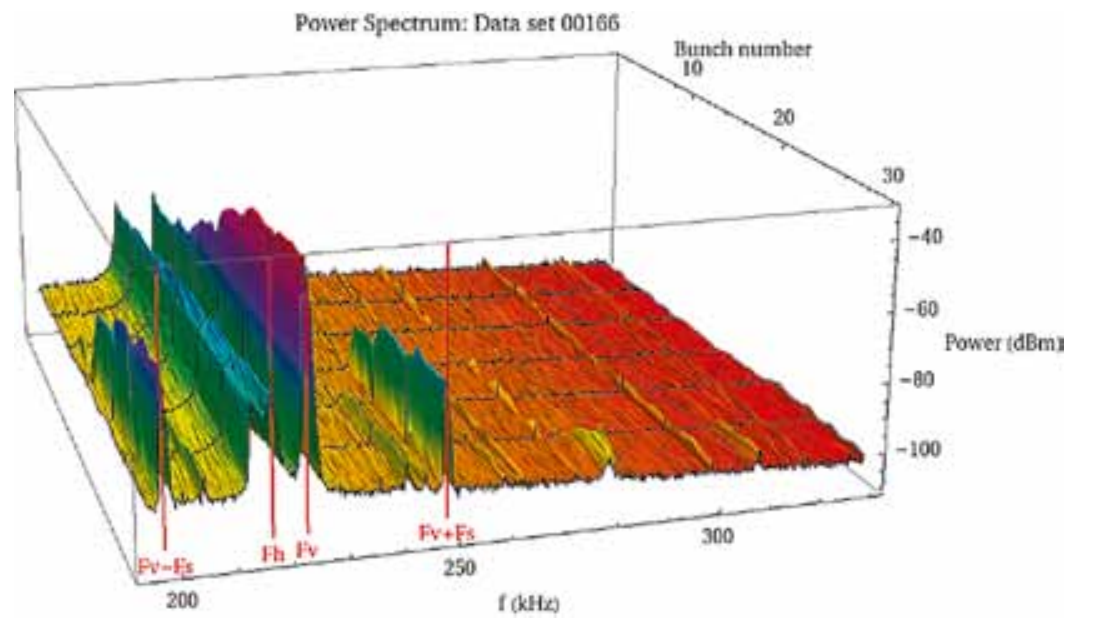

Figure 3.8 Bunch-by-bunch beam sizes based on turn-by-turn fits for each bunch for 30 bunch trains of varying current $\left(0.8,1.2\right.$, and $1.6 \times 10^{10}$ particles/ bunch). As the bunch currents are increased, the point in the train at which the electron cloud density is high enough to cause emittance and beam size growth moves to earlier points in the train.
A second observable associated with this instability is a growth in the vertical beam size as measured along the train. Figure 3.8 shows bunchby-bunch beam size development along bunch trains with three different intensities. As the bunch currents are increased, the bunch number in the train at which beam size blow-up occurs moves earlier in the train due to the more rapid build-up of the electron cloud. By studying both the spectral and beam size information as a function of various parameters (bunch intensity, vertical emittance, bunch spacing, chromaticity, feedback conditions, and beam energy) and comparing with simulation [3-37, 38], we will be able to validate the simulations in a regime approaching that of the ILC damping ring to ensure that our projections of the expected positron damping ring performance are accurate. 
Incorporation of CesrTA results into the ILC damping ring technical design

The results from the first two-and-a-half years of the CesrTA R\&D

programme are currently being integrated into the ILC damping ring technical design [3-39]. In particular, the observed efficacy of grooved chamber surfaces in the dipoles as well as that of the clearing electrode in the high-field wigglers provide confidence that practical electron cloud mitigations can be prepared for the arc and wiggler straight regions of the ILC positron damping ring. The importance of cloud mitigation in the damping ring quadrupole chambers has also been demonstrated. New coating technologies to suppress the secondary electron yield offer great promise. However, there is still the issue of studying the long-term performance and durability of these coatings. This will be a subject of study during Phase II of the CesrTA programme. Perhaps most importantly, the flexibility of CESR operations supports a systematic programme of electron cloud build-up and electron cloud-induced beam-dynamics studies. By benchmarking our physics models and simulations against these studies, our confidence in being able to make valid projections of the expected ILC positron damping performance has been significantly enhanced.

\subsubsection{Electron cloud R\&D at other laboratories}

During 2007 and 2008 in the Positron Low Energy Ring of the PEP II

accelerator, a magnetic chicane and special vacuum chambers were installed to study electron cloud effects in an accelerator beamline [3-40, 41]. A special chamber was used to monitor the secondary electron yield of titanium nitride and titanium-zirconium-vanadium (NEG) coating, copper, stainless steel and aluminium under the effect of electron and photon conditioning in situ in the beam line. A drastic reduction of the secondary electron yield to approximately 0.95 for titanium nitride and a still-high value for aluminium of greater than 2.0 after exposure in the accelerator beamline has been measured. Other vacuum-chamber materials including NEG coated samples have also been measured. In magnetic field-free regions, chambers have been installed with rectangular groove profiles meant to reduce the secondary electron generation at the surface. The electron signals in the grooved chambers, when compared to signals in smooth chambers, were significantly reduced. From the electron cloud chicane tests, two important results in dipoles are reported: 1) the titanium nitride coating reduces the cloud density by several orders of magnitude with respect to a bare aluminium surface and 2) a new resonance phenomenon has been observed that results in the modulation of the electron wall flux, and hence, one presumes, of the electron cloud density. After the PEP II shutdown the magnetic chicane and the test chambers were installed in the CesrTA ring (see section 3.1.2) to continue the cloud mitigation studies.

Tests of coated chambers, grooves and clearing electrodes have been carried out at KEK in order to mitigate the electron cloud instability in an intense positron ring $[3-26,42,43,44]$. Aiming for the application in a dipole-type magnetic field, various shapes of triangular grooved surfaces have been studied. In a laboratory, the secondary electron yields of small test pieces were measured using an electron beam in the absence of magnetic fields. The grooved surfaces clearly had low secondary electron yield compared to flat surfaces of the same materials. The grooves with sharper vertices had smaller secondary electron yield. 
A test chamber installed in a wiggler magnet of the KEK-B positron ring was used to investigate the efficacy of the grooved surface in a strong magnetic field. In the chamber, a remarkable reduction in the electron density around the beam orbit was observed compared to the case of a flat surface with titanium nitride coating.

An electron-clearing electrode with an ultra-thin structure has been developed. The electrode was tested with a positron beam of the KEK-B. A drastic reduction in the electron density around the beam was demonstrated in a wiggler magnet with a dipole-type magnetic field of 0.78 tesla (T). No discharge or extra heating of the electrodes and feedthroughs was observed after using the latest connection structure. The same type of electrode was also successfully tested in a CesrTA wiggler (see section 3.1.2). The clearing electrode has also been applied to a copper beam pipe with antechambers in preparation for its application in the wiggler section of Super-KEKB. Simulations indicate a small impedance for the thin structure of this electrode design.

At the INFN Frascati National Laboratories in Italy, clearing electrodes to mitigate the electron cloud instability have been installed in all the dipole and wiggler chambers of the DAФNE positron ring, covering approximately $16 \%$ of the circumference [3-45]. All the electrodes have been inserted, leaving the chambers in place. Tests of the electrodes' effectiveness at high positron current will be done shortly.

At CERN, amorphous carbon thin films have been applied to the liners in the electron cloud monitors and to vacuum chambers of three dipole magnets in the SPS [3-27]. The electron cloud is completely suppressed for LHC-type beams in the liners even after three months of air venting, and no performance deterioration is observed after one year of SPS operation. Following the positive preliminary results obtained at the SPS it was decided to test these types of coatings in a high synchrotron radiation environment in a lepton machine at CesrTA (see section 3.1.2).

\subsubsection{Preliminary recommendations of the ILC Electron Cloud Working Group} A working group has been set up to evaluate the electron cloud effect and instability issues for the ILC positron damping ring and to recommend mitigation solutions. The collaborating institutions are Argonne National Laboratory, CERN, Cornell University, INFN, KEK, Lawrence Berkeley National Laboratory and SLAC. The first task of the working group was to compare the electron cloud effect for two different damping ring designs with 6.4-kilometre $(\mathrm{km})$ and 3.2-km circumferences, respectively, and to investigate the feasibility of the shorter damping ring with respect to the electron cloud build-up and related beam instabilities. We compared the instability thresholds and the electron cloud formation assuming 6-nanosecond (ns) bunch spacing in both configurations, that is, in the same beam current. Both ring configurations were found to exhibit very similar performances. The risk associated with the adoption of the 3.2-km damping ring design, while maintaining the same bunch spacing, was deemed low and the $3.2-\mathrm{km}$ ring was found to be an acceptable baseline design choice. 


\begin{tabular}{|c|c|c|c|c|}
\hline \multirow{2}{*}{$\begin{array}{l}\text { Field region } \\
\text { Drift }^{*}\end{array}$} & \multicolumn{2}{|c|}{ Baseline mitigation recommendation } & \multirow{2}{*}{$\begin{array}{l}\text { Alternatives for further investigation } \\
\text { NEG coating }\end{array}$} & \multirow{3}{*}{$\begin{array}{l}\text { Table } 3.3 \text { Summary of baseline electron cloud } \\
\text { mitigation recommendations developed at the } \\
\text { Electron Cloud Working Group meeting held as part } \\
\text { of the ECLOUD10 Workshop on } 13 \text { October } 2010 \text {. }\end{array}$} \\
\hline & TiN coating & Solenoid windings & & \\
\hline Dipole & $\begin{array}{l}\text { Grooves with } \\
\text { TiN coating }\end{array}$ & $\begin{array}{l}\text { Antechambers for } \\
\text { power loads and } \\
\text { photoelectron control }\end{array}$ & $R \& D$ in the use of clearing electrodes & \\
\hline Quadrupole* $^{*}$ & TiN coating & & $\begin{array}{l}\text { R\&D in the use of clearing electrodes or } \\
\text { grooves with TiN coating }\end{array}$ & $\begin{array}{l}\text { * Where drift and quadrupole chambers are in arc or } \\
\text { wiggler straight regions of the machine, the }\end{array}$ \\
\hline Wiggler & $\begin{array}{l}\text { Clearing } \\
\text { electrodes }\end{array}$ & $\begin{array}{l}\text { Antechambers for } \\
\text { power loads and } \\
\text { photoelectron control }\end{array}$ & Grooves with TiN coating & $\begin{array}{l}\text { chambers will incorporate features of those } \\
\text { sections, that is, antechambers for power loads } \\
\text { and photoelectron control. }\end{array}$ \\
\hline
\end{tabular}

The preliminary mitigation recommendations for the ILC damping rings are the result of the working group discussions held during a number of workshops and regular online meetings. The working group met at Cornell University on 13 October 2010 as a satellite meeting to the ECLOUD10 Workshop held from 8 to 12 October. The workshop was devoted to hearing the results of detailed studies of a range of mitigation options. Input from the workshop participants was included in the evaluation. The results of the evaluation were presented at the IWLC2O1O workshop at CERN [3-46]. Table 3.3 provides a brief summary of the recommendations. 


\subsection{THE ATF2 FINAL FOCUS} TEST BEAMLINE AT KEK

\subsubsection{Introduction}

The challenge of colliding nanometre-sized beams at the interaction point involves three distinct issues:

- creating small emittance beams.

- preserving the emittance during acceleration and transport.

- focusing the beams to nanometres before colliding them.

The Accelerator Test Facility (ATF) at KEK in Japan is a prototype damping ring, which has succeeded in obtaining emittances that come close to satisfying ILC requirements. ATF is now used as a beam injector for the ATF2 final focus test beamline, constructed in 2008. The primary goals for ATF2 address the challenge of the beam size:

- achieving a 37-nanometre $(\mathrm{nm})$ vertical beam size at the interaction point.

- stabilising the beam at that point at the nanometre level.

The main parameters of ATF2 are given in Table 3.4, together with the corresponding values for the ILC.
Table 3.4 Main design parameters for ATF2 compared with those for ILC. The ATF2 37-nm beam size (at the interaction point) includes residual effects from uncorrected higher-order optical aberrations.

\begin{tabular}{|c|c|c|c|c|}
\hline Parameter & & & ATF2 & ILC \\
\hline Beam energy & E & $\mathrm{GeV}$ & 1.3 & 250 \\
\hline Effective focal length & $L^{*}$ & $\mathrm{~m}$ & 1 & $3.5-4.5$ \\
\hline Horizontal emittance & $\varepsilon_{\mathrm{x}}$ & $\mathrm{nm}$ & 2 & 1.0 (damping ring) \\
\hline Vertical emittance & $\varepsilon_{\mathrm{y}}$ & $\mathrm{pm}$ & 12 & 2 (damping ring) \\
\hline Horizontal IP $\beta$ function & $\beta^{*}$ & $\mathrm{~mm}$ & 4 & 21 \\
\hline Vertical IP $\beta$ function & $\beta_{y}^{*}$ & $\mathrm{~mm}$ & 0.1 & 0.4 \\
\hline Horizontal IP angular dispersion & $\eta^{\prime}$ & & 0.14 & 0.0094 \\
\hline Relative energy spread & $\sigma_{\mathrm{E}}$ & $\%$ & $\sim 0.1$ & $\sim 0.1$ \\
\hline Chromaticity & & & $\sim 10^{4}$ & $\sim 10^{4}$ \\
\hline RMS horizontal beam size & $\sigma^{*}$ & $\mu \mathrm{m}$ & 2.8 & 0.655 \\
\hline RMS vertical beam size & $\sigma_{y}^{*}$ & $\mathrm{~nm}$ & 37 & 5.7 \\
\hline
\end{tabular}

The layout of the ATF-ATF2 facility and the design optical functions of the ATF2 beamline are displayed in Figures 3.9 and 3.10, respectively. The optics system - based on a local chromatic correction scheme that affords a compact geometry - is a scaled-down version of the ILC design. 


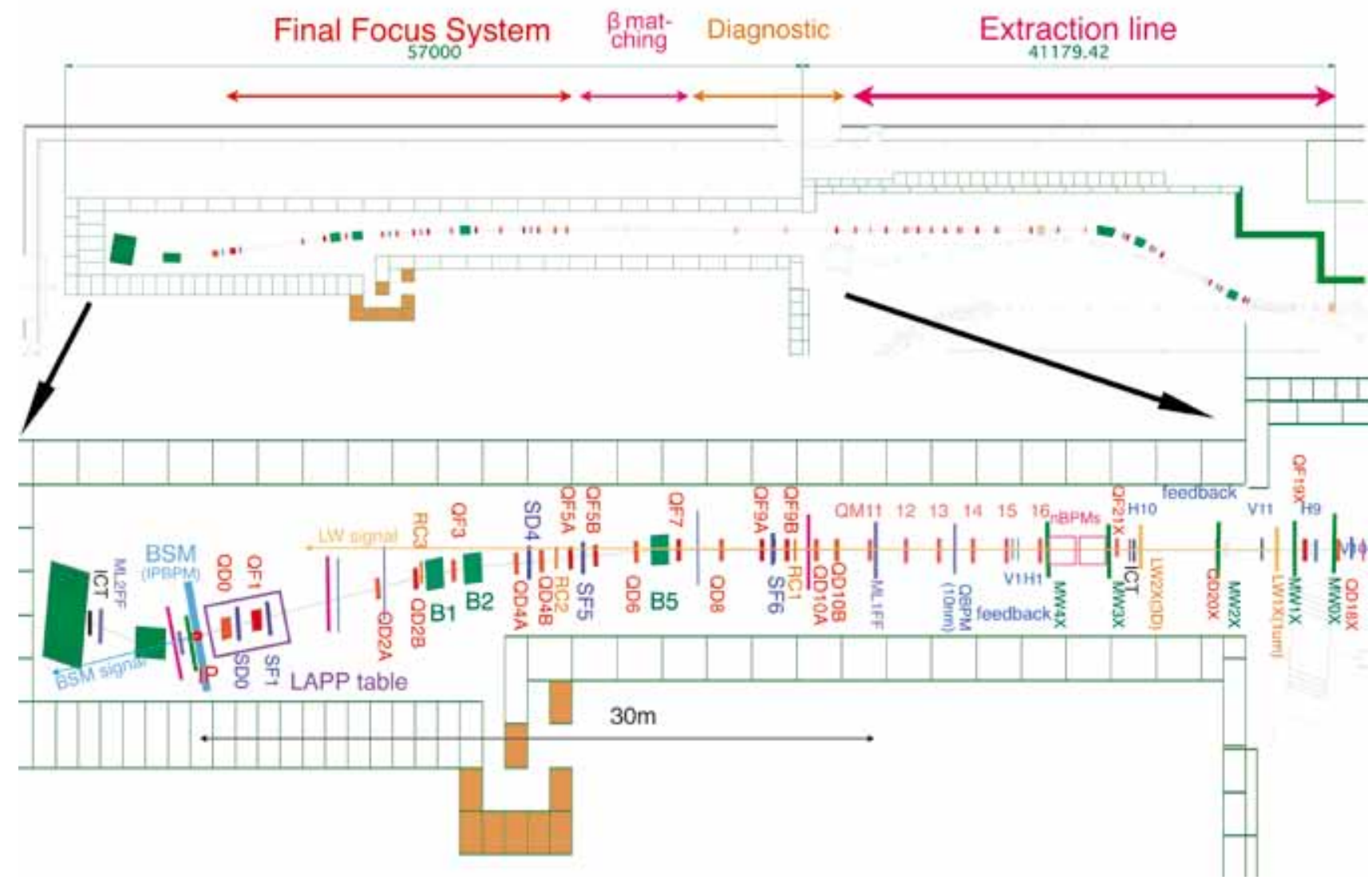

Figure 3.9 Top: the ATF2 beam line. Bottom: an enlargement of the final focus system.

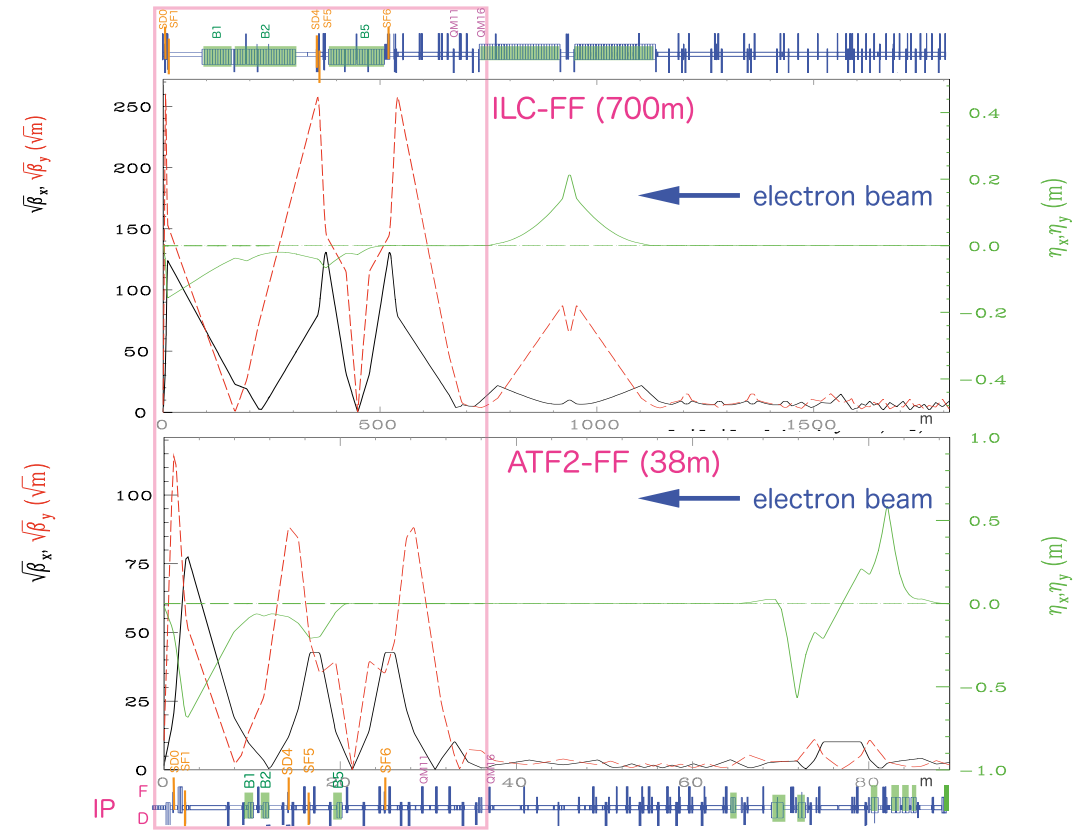

Figure 3.10 Top: ILC beam delivery system optics from the exit of main linac on the right to the interaction point on the left. Bottom: ATF2 optics from the ATF damping ring extraction point on the right to the interaction point on the left. 


\subsubsection{Status of ATF2 systems}

\section{Magnets and magnet mover}

The ATF2 beamline extends over about $90 \mathrm{~m}$ from the beam extraction point in the ATF damping ring to the interaction point (see Figures 3.9 and 3.11). Many quadrupoles and some dipoles were fabricated for ATF2 by IHEP in China, while others were reused from the old ATF extraction beamline and from the Final Focus Test Beam at SLAC. Among those from the latter were the two quadrupole and two sextupole magnets that make up the strong-focusing final doublet system just before the interaction point. The apertures of the final doublet quadrupole magnets needed to be increased to accommodate the larger $\beta$ function values in the ATF2 optics design.

Figure 3.11 View looking downstream along the final focus section of the ATF2 beamline.

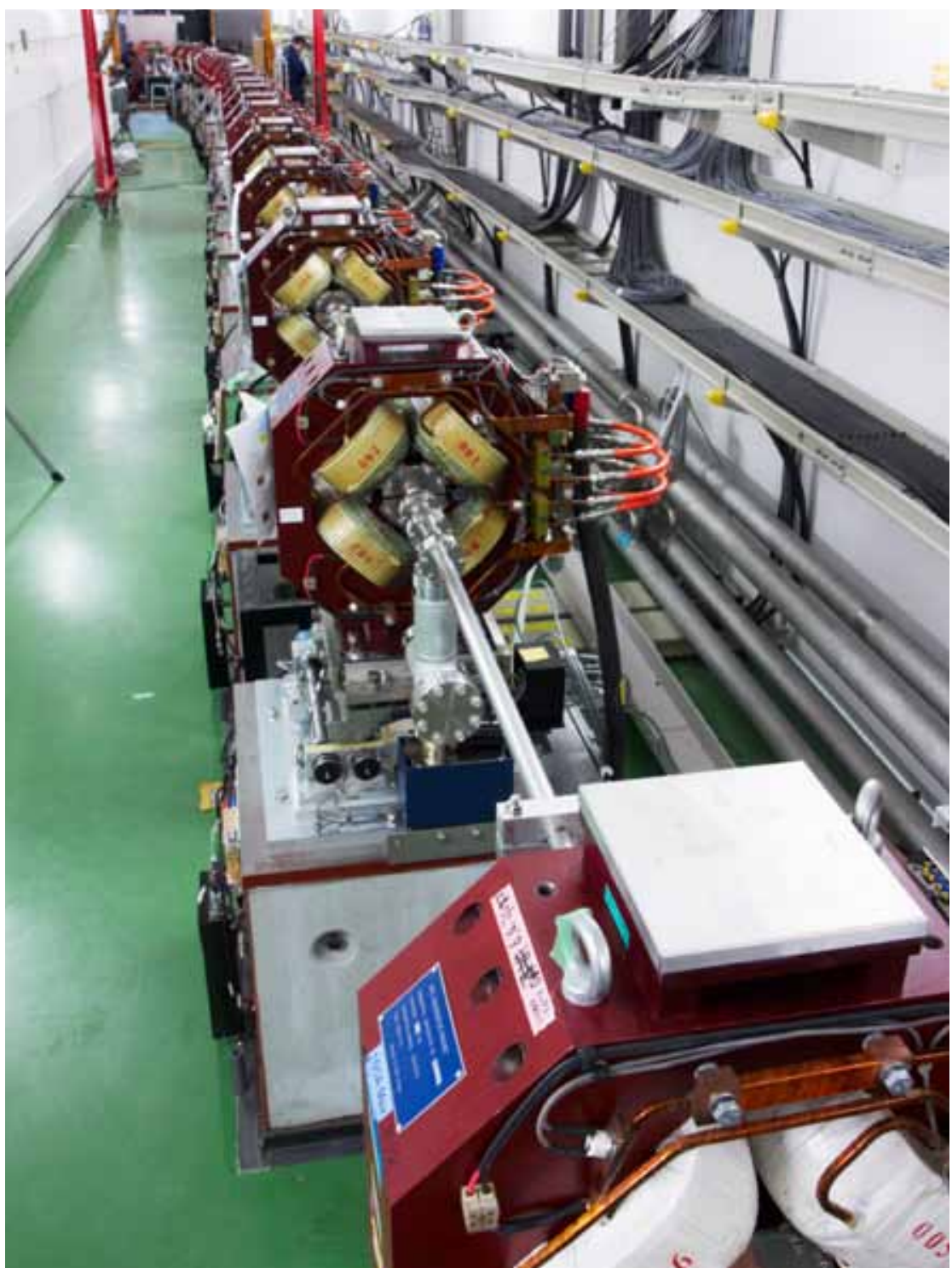


Anticipating gradual movements of supports and magnets due to thermal variations or slow ground motion, 20 quadrupole and five sextupole magnets in the final focus were mounted on remote-controlled three-axis movers recycled from the Final Focus Test Beam experiment. The movers have a precision of 1 to 2 micrometres $(\mu \mathrm{m})$ for transverse motion (horizontal and vertical), and 3 to 5 microradians ( $\mu \mathrm{rad}$ ) for rotations about the beam axis.

Overall alignment precisions of o.1 millimetres $(\mathrm{mm})$ (displacement) and o.1 milliradians (mrad) (rotations) have been achieved using conventional alignment and metrology techniques. The final alignment of the magnets is achieved via beam-based alignment techniques.

\section{Final doublet}

The final doublet is composed of two quadrupole and two sextupole magnets (labelled QDo, QF1, SDo, SF1 in Figure 3.9). These magnets must be supported in a way that ensures that their vertical vibration amplitude relative to the interaction point is smaller than $7 \mathrm{~nm} \mathrm{rms}$ above 0.1 hertz (Hz); this limits unwanted effects on the measured beam size at the interaction point to less than $5 \%$ of the total size. For vibrations below $0.1 \mathrm{~Hz}$, beam-based feedback methods can be used to limit those effects. A rigid support was chosen since it strongly suppresses the relative motion of the final doublet and the interaction point. Vibration measurements with the table fixed to the floor and with all magnets and movers installed were performed in the laboratory for validation, including checking for potential effects from cooling water flowing in the magnets. Additional measurements after installation of the final doublet confirmed that the residual motions of the magnets relative to the interaction point were within tolerance. The whole final doublet system is shown in Figure 3.12.



Figure 3.12 View of the final doublet installed on its rigid mechanical support system. 


\section{Cavity beam position monitors}

The ATF2 beamline is instrumented with $32 \mathrm{C}$-band (6.5 gigahertz) and four S-band $(2.8 \mathrm{GHz})$ high-resolution cavity beam position monitors, fabricated by Pohang Accelerator Laboratory and Kyungpook National University in Korea. There are also four C-band and one S-band reference cavities to monitor beam charge and beam arrival phase. In the diagnostics and final focus sections, every quadrupole and sextupole magnet is instrumented with a cavity beam position monitor. The final doublet magnets use S-band beam position monitors, while the other quadrupoles are equipped with C-band monitors. The usable measurement range of the cavity beam position monitors was found to exceed the mechanical range of quadrupole movers $( \pm 1.5 \mathrm{~mm})$. A resolution of 200 to $400 \mathrm{~nm}$ for the C-band beam position monitors has so far been demonstrated.

\section{Interaction point beam size monitor}

Measuring transverse beam sizes of tens of nanometres at the interaction point requires specialised beam instrumentation, in particular a laser interferometer-based beam size monitor, also referred to as a Shintake monitor. This beam size monitor is based on inverse Compton scattering between the electron beam and a laser interference fringe pattern.

For the ATF2 beam energy, the energy of the generated gamma rays is typically rather low compared to the main component of detector backgrounds, Bremsstrahlung photons (these are emitted when electrons in the transverse tails of the beam interact with apertures and start showering). In the monitor designed for ATF2, the signal is separated from this high-energy background by analysing the signal's longitudinal shower profile, measured with a multilayered detector (located a few metres after the interaction point, downstream from a dipole magnet). The laser wavelength is $532 \mathrm{~nm}$, the second harmonic of the $\mathrm{Nd}$ :YAG (neodymium-doped yttrium aluminium garnet) laser, which provides a suitable fringe pitch to measure the target vertical size of $37 \mathrm{~nm}$. Four laser beam crossing modes of 2-, 8-, 30- and 174-degree angles are available to provide a broad dynamic range of up to several micrometres, allowing the initial beam size to be tuned down to the nominal beam size or smaller. In addition, a single 'laser wire' mode can be used for horizontal beam size measurements.

\section{Other beam line instrumentation}

The instrumentation from the old ATF extraction line (strip line beam position monitors, integrated current transformers, optical transition radiation, screen profile monitors and wire scanners) is reused in the reconfigured beamline. There are five wire scanners with tungsten and carbon wires of $10-\mu \mathrm{m}$ and $7-\mu \mathrm{m}$ diameters, respectively, located in the diagnostic section upstream of the final focus section (see Figure 3.9). They are used to measure the horizontal and vertical beam emittances after extraction from the damping ring. An additional wire scanner is installed just downstream of the interaction point for beam size tuning and has tungsten and carbon wires of $10-\mu \mathrm{m}$ and $5-\mu \mathrm{m}$ diameters, respectively. Screen monitors are located right after the extraction, in the middle of the beamline and before and after the final doublet. An optical-fiber beam loss monitor is installed all along the beamline to measure and localise beam losses. Four optical transition radiation monitors with an improved resolution of 2- $\mu \mathrm{m}$ have been installed in the extraction line, close to the wire scanners. These monitors are used for single-bunch beam size as well as fast emittance measurements. 


\section{Commissioning status}

EMITTANCE IN THE DAMPING RING

Figure 3.13 shows recent results of the vertical emittance measured by three

different devices at the damping ring. The vertical emittance of $10 \mathrm{pm}$

required for ATF2 was routinely obtained with the standard beam tuning

procedures for the damping ring.

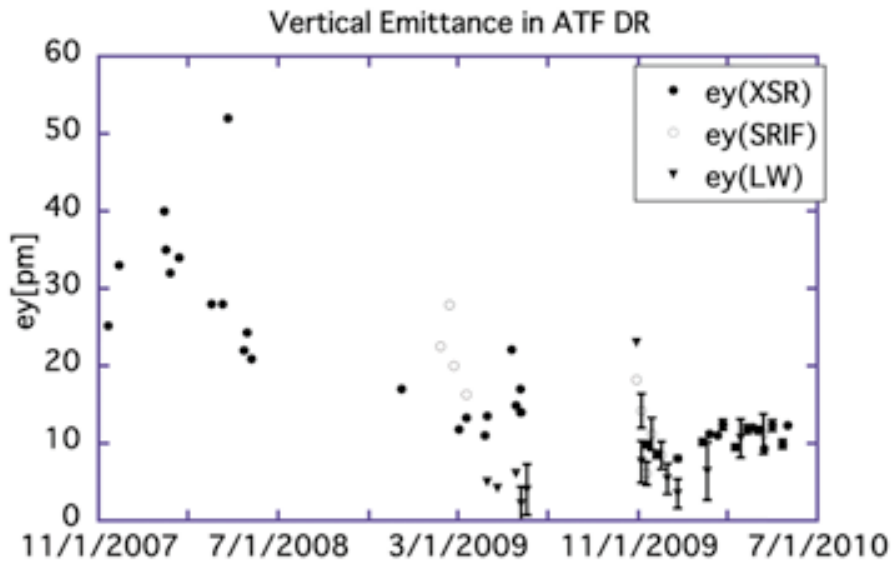

Figure 3.13 Recent measurements of vertical

emittance at the damping ring, where XSR, SRIF

and LW are values by X-ray profile of synchrotron

radiation, interference pattern of synchrotron

radiation and the laser wire, respectively.

\section{BEAM TUNING STRATEGY}

Focusing the low-emittance beam extracted from the ATF damping ring to the specified interaction point beam size requires correcting trajectory and optics distortions induced both by imperfections along the beamline and by mismatch of the beam phase space at damping ring extraction. While final corrections must be performed at the interaction point, it is still important to keep mismatches under control at the entrance of the final focus in order to limit distortions of the linear optics in the carefully tuned chromatic correction section. It is also important for minimising Bremsstrahlung backgrounds in the beam size monitor. These arise from electrons driven to large amplitudes, which then cause showers at the limiting apertures.

To focus the low-emittance beam, all the magnets except for the dipoles must first be aligned with respect to the beam using the beam position monitors. In the final focus section, the positions of most magnets were adjusted using their mechanical movers; steering magnets were used to centre the beam in the upstream magnets. In the extraction line, two quadrupole magnets (labelled QF1X and QF6X - see Figure 3.9) and two skew-quadrupole magnets (labeled QS1X and QS2X) were used to correct horizontal and vertical dispersions at the end of the extraction line.

There are five sextupole magnets in the final focus system (SF6FF, SF5FF, SDFF, SF1FF and SDOFF), which are primarily required for correction of chromatic and geometric optics aberrations. By adjusting linear combinations of the horizontal and vertical displacements of these magnets using their magnet movers, the beam waist positions, dispersions and crossplane coupling at the interaction point can be orthogonally tuned. 
Figure 3.14 The beam position monitor model (lattice) response measured by steering beam at a horizontal corrector magnet ZH4X (top) and at a vertical corrector magnet $\mathrm{ZV} 4 \mathrm{X}$ (bottom) in the extraction line. Both measured and simulated data are shown.
The final doublet quadrupole strengths are also used for fine-tuning. The primary tuning diagnostic is the beam size monitor together with the mechanical wire beam size measurements at the interaction point.

\section{OVERVIEW OF COMMISSIONING RUNS}

Commissioning ATF2 with beam began in late 2008 and continued throughout 2009. Most of the early commissioning was focused on hardware and software commissioning. To reduce the beam size in the final doublet, and therefore background rates, during initial commissioning of the instrumentation (most notably the beam size monitor), the initial optics used a lower demagnification than the goal optics. Interaction point $\beta$ functions of 8 centimetres $(\mathrm{cm})$ in both the horizontal and vertical planes - factors of 20 and 800 higher, respectively, than design - were used. Under these conditions, initial interaction point beam sizes of $12.5 \mathrm{~mm}$ (horizontal) and 1 to $2 \mathrm{~mm}$ (vertical) were measured with the beam size monitor in laser wire mode.

After this initial success, the vertical interaction point $\beta$ function was reduced from $8 \mathrm{~cm}$ to $1 \mathrm{~cm}$, corresponding to a theoretical vertical beam size of approximately $500 \mathrm{~nm}$. (The horizontal $\beta$ function was left unchanged.) Further development of the instrumentation at this time included additional interaction point diagnostics (a screen monitor, wire scanners and a knife-edge monitor) and an upgrade to the interaction point beam size monitor, replacing the laser with a more powerful one (four times greater in intensity). With the stronger focusing optics, the first detailed measurements of the lattice response and optics functions were made (Figure 3.14). Towards the end of 2009 another major milestone was achieved with the first beam size monitor measurements in interference mode, where a vertical beam size of $3.3 \mathrm{~mm}$ in the 3-degree crossing mode was observed.

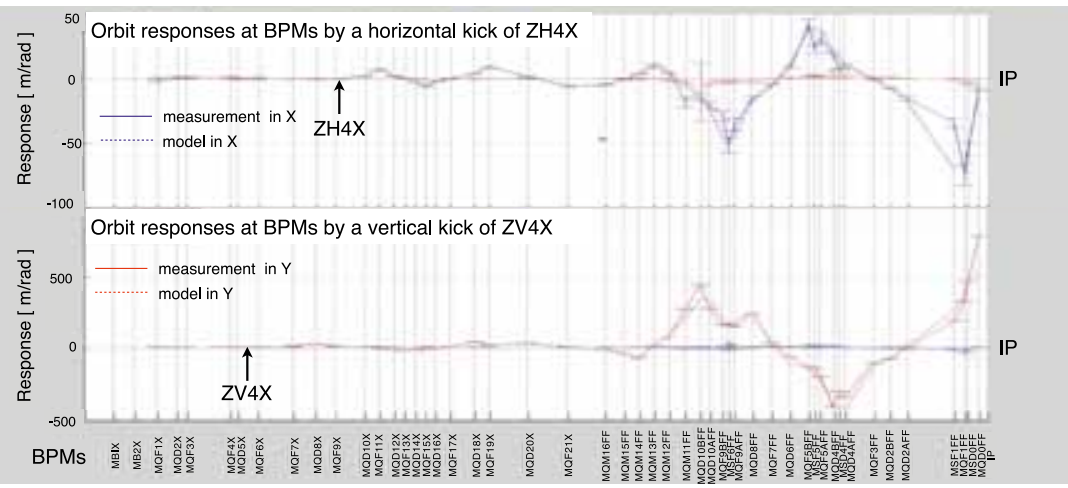

In early 2010, another significant step in demagnification was made with the interaction point $\beta$ functions now only $1 \mathrm{~mm}$ high and $4 \mathrm{~cm}$ wide (ten times larger than the design). This level of demagnification required the use of the sextupoles for the first time to correct the chromatic aberration at the interaction point. With the beam size monitor and beam position monitor systems now fully commissioned, application of the interaction point beam size tuning algorithms could be made for the first time, squeezing the beam size down to the expected value. 
Figure 3.15 shows the raw beam size monitor measurement, corresponding to a vertical beam size of $310 \pm 30$ (statistical) \pm 30 (systematic) $\mathrm{nm}$ - almost three times the value of the expected size of $110 \mathrm{~nm}$, with a vertical emittance of $12 \mathrm{pm}$. Two possible sources of error were identified: alignment roll error of the final doublet quadrupoles, and insufficient commissioning time for the interaction point beam size (Shintake) monitor using the 30-degree mode required for beam size measurements less than $300 \mathrm{~nm}$. The final doublet roll has been subsequently confirmed by mechanical inspection and realigned. One major issue for small beam size operation is high-order multipole components (aberrations) in the quadrupole magnets. A retuned optics has been proposed to mitigate the (simulated) effects of these multipole components. Currently, a new optics is being implemented with the design demagnification in the vertical plane (vertical interaction point $\beta$ function of $0.1 \mathrm{~mm}$ ), but with a still relaxed horizontal demagnification by a factor of 2.5 (horizontal interaction point $\beta$ function of $10 \mathrm{~mm}$ ). The optics includes the proposed mitigation for the quadrupole multipole components.

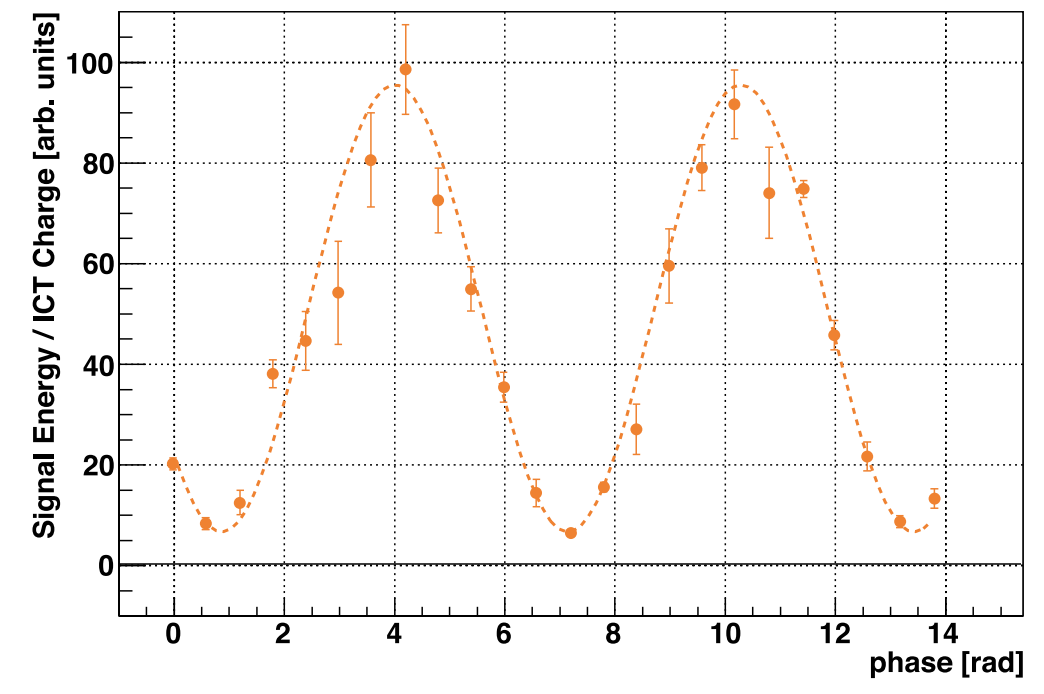

Figure 3.15 The best modulation measured to date by the beam size monitor (Shintake monitor) with the 8-degree crossing angles in the continuous run, taken May 2010. The measurement corresponds to a beam size of $310 \pm 30$ (statistical) \pm 30 (systematic) $\mathrm{nm}$. 
Figure 3.16 Recent results of feedback on a nanosecond time scale. The above three plots are experimental results. The bottom one is a simulated result to demonstrate the nanometre stabilisation at the interaction point, assuming a perfect lattice in the final focus beam line.

\subsubsection{ATF2 outlook and plans}

The remainder of Technical Design Phase 2 (and beyond) will see a concerted effort to achieve the goal of $37-\mathrm{nm}$ vertical beam size. In parallel to this, several R\&D activities related to the second ATF2 goal - stability - are being actively pursued:

- feedback on a nanosecond time scale

- nanometre resolution of the interaction point beam position monitor

- fast nanosecond rise-time kicker

- cavity beam position monitor optimised for monitoring angular variations of the beam near the interaction point with high accuracy

- development of robust laser wire diagnostics

The most recent results of feedback on a nanosecond (ns) time scale are shown in Figure 3.16, where a measurement of the beam offset at the first of a three-bunch train is used to correct (feed back on) the subsequent two bunches. Bunch separation is $151.2 \mathrm{~ns}$. The data clearly indicates a reduction of the beam jitter by a factor of five from the first to the second bunch. The achieved 2.1- $\mu \mathrm{m}$ rms scales to $2.6 \mathrm{~nm}$ at the interaction point, assuming the demagnification of the optics.

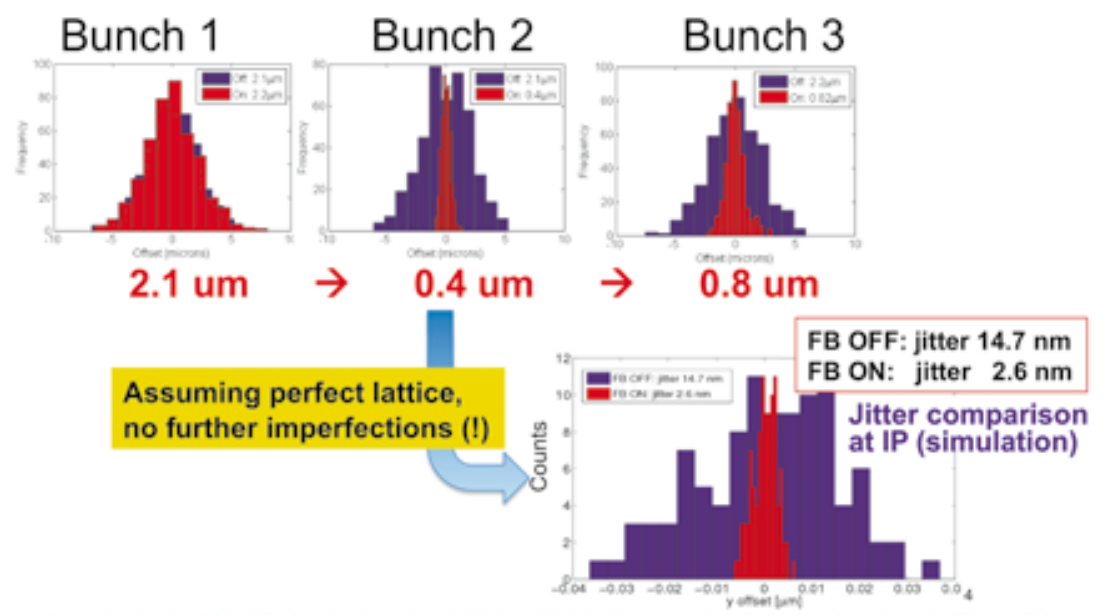

Plans to upgrade the performance of ATF2 on the time scale of a few years, after the main goals of ATF2 have been achieved, are also under consideration. In particular, optical configurations with ultralow $\beta^{*}$ values (two to four times smaller than the current nominal values in the horizontal and vertical planes), relevant to both the CLIC design and to some of the alternative ILC beam parameter sets, are actively studied. In order to allow beam-based stability studies, there is also a proposal to upgrade the final doublet with superconducting magnets built using the foreseen ILC directwind technology. An R\&D programme to develop a tunable permanent magnet suitable for the final doublet is also pursued in parallel, with an initial goal to construct a prototype for initial beam testing in the upstream part of the ATF2 beamline. 


\subsubsection{Polarised electron source}

Currently, the R\&D for the ILC polarised electron source focuses on two aspects. The first is to build a prototype of the source laser system with the goal of generating an electron beam with ILC beam parameters (Table 3.5). For this demonstration of the ILC beam, SLAC's ILC Injector Test Facility will be used. This facility comprises the laser facility, the US Stanford Linear Collider electron gun with associated diagnostics, including a Faraday cup for bunch charge, and a Mott polarimeter for electron polarisation measurements. At a later stage, it is planned to move the laser system to US Jefferson Laboratory's injector facility to allow beam demonstration with a higher-voltage electron gun (16o to 200 kilovolts $(\mathrm{kV}))$, which is currently under construction.

The second aspect of R\&D is aimed at the electron gun itself. The goals are to achieve the ILC specification for a gun voltage of $200 \mathrm{kV}$ while maintaining a low dark current to ensure a long cathode lifetime.

\subsection{ACCELERATOR}

SYSTEMS R\&D

\begin{tabular}{|c|c|c|}
\hline Parameter & Symbol & Value \\
\hline Number of electrons per bunch at gun exit & $\mathrm{n}_{\mathrm{e}}$ & $4 \times 10^{10}$ \\
\hline Number of electrons per bunch at damping ring injection & $\mathrm{n}_{\mathrm{e}}$ & $2 \times 10^{10}$ \\
\hline Number of bunches & $\mathrm{N}_{\mathrm{e}}$ & 2,820 \\
\hline Bunch repetition rate & $\mathrm{F}_{\mu \mathrm{b}}$ & $3 \mathrm{MHz}$ \\
\hline Bunch train repetition rate & $\mathrm{F}_{\mathrm{mb}}$ & $5 \mathrm{~Hz}$ \\
\hline Bunch length at source & $\Delta \mathrm{t}$ & $2 \mathrm{~ns}$ \\
\hline Peak current in bunch at source & $I_{\text {avg }}$ & $3.2 \mathrm{~A}$ \\
\hline Energy stability & $\mathrm{S}$ & $<5 \% \mathrm{rms}$ \\
\hline Polarisation & $\mathrm{Pe}$ & $\geq 80 \%$ \\
\hline Photocathode quantum efficiency & $\mathrm{QE}$ & $0.5 \%$ \\
\hline Drive laser wavelength & $\Lambda$ & $780-810 \mathrm{~nm}$ (tunable) \\
\hline Single-bunch laser energy & $\mathrm{E}$ & $5 \mu \mathrm{J}$ \\
\hline
\end{tabular}

Table 3.5 ILC electron source beam parameters.

Currently, no photocathode R\&D is being conducted. Results from previous R\&D projects have demonstrated that materials are available that can provide the ILC beam charge and polarisation. It is anticipated that the ILC source will use a strained gallium arsenide phosphide (GaAsP) highly-doped photocathode. Figure 3.17 illustrates the performance of such a cathode. The last remaining question is the surface charge limit at microsecond timescales. The laser system currently under development will answer this question. 
Figure 3.17 Performance of strained layers of GaAsP photocathodes at different doping levels.

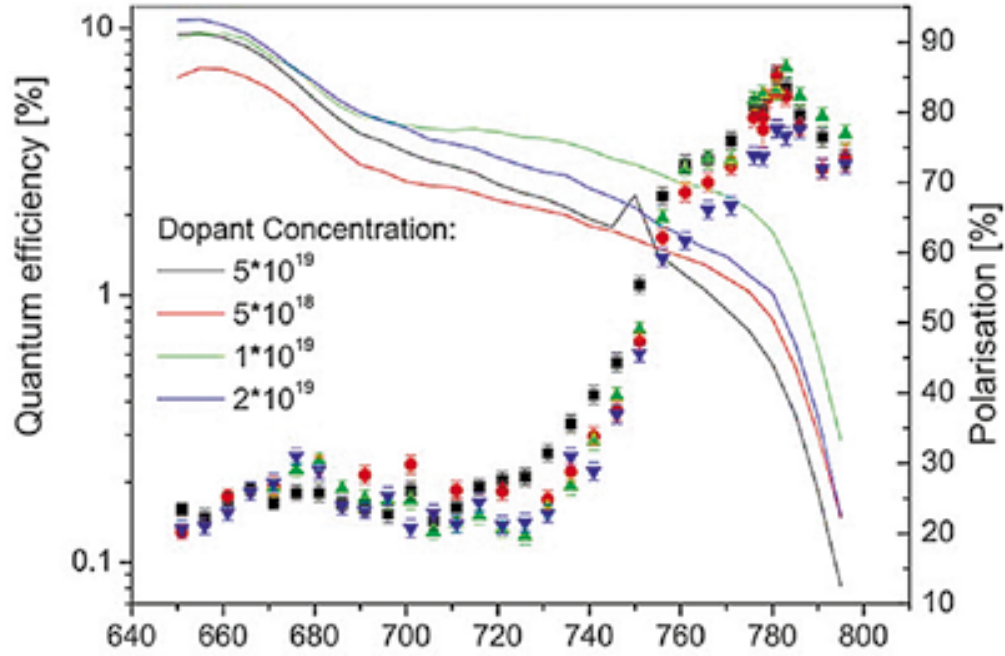

Wavelength [nm]
Laser system development

A laser system is being developed for the ILC polarised injector that is capable of generating the ILC bunch train. The laser wavelength must match the band gap of the cathode material. For GaAsP, a wavelength of approximately $800 \mathrm{~nm}$ is necessary. The laser system must provide the time structure of the ILC pulse train. One basic component of this laser system is the mode-locked oscillator that operates at a harmonic frequency of the micro-bunch repetition rate, which can be locked to an external reference frequency. For efficient amplification of the pulse train, a regenerative amplifier is used (Figure 3.18).
Figure 3.18 Optical layout of the regenerative amplifier.

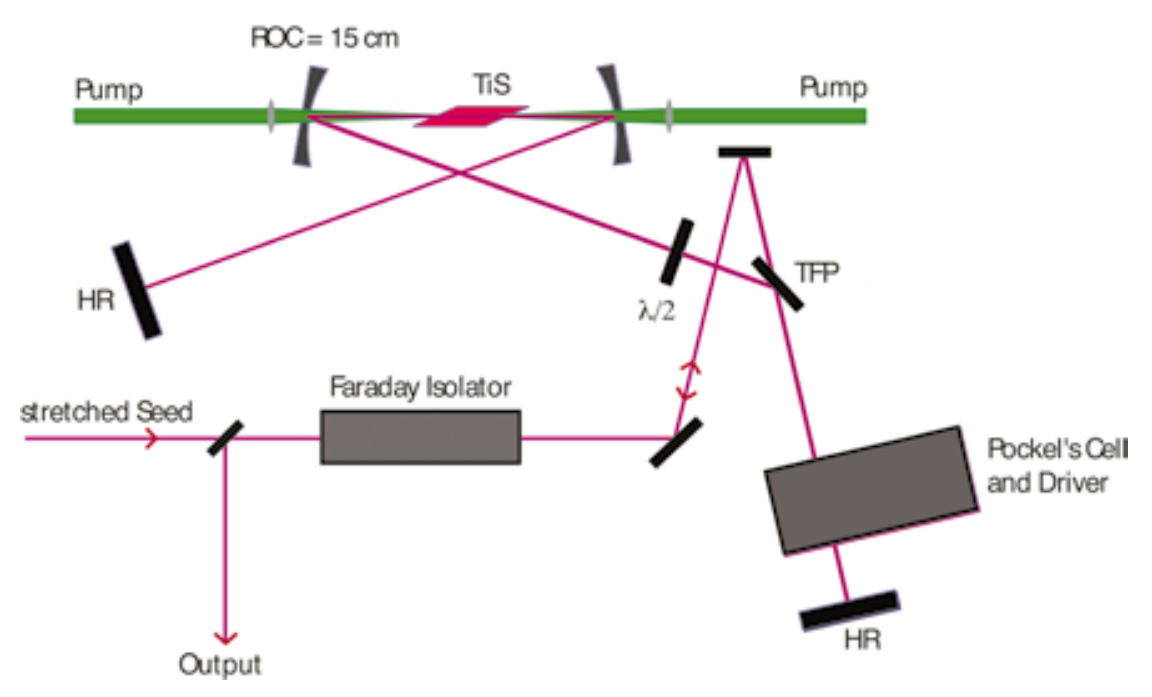


A key component of this amplifier is the cryogenic cell containing a titanium-sapphire crystal. Cryogenic technology allows large pump power and efficient amplification, minimising the effects of thermal lensing in the amplifying medium. The micro-bunch structure (3-megahertz $(\mathrm{MHz})$ pulse train) is controlled by injection and extraction of the regenerative amplifier using a high-repetition-rate Pockel's cell system. The macro-bunch structure ( 5 to $10 \mathrm{MHz}$ ) is generated by electro-optical switching of the amplified beam. At SLAC, two such laser systems are being built: a SLAC version and a second similar system that was constructed by a commercial laser company, Kaptayne Murnane Laboratories, Inc.

\section{Direct current gun}

The main goal of R\&D towards a direct current gun for polarised electron generation is to increase the high-voltage capability while maintaining or reducing the dark current. A higher voltage is desirable to reduce the space charge forces that the electrons experience at low energy before further acceleration. The reduction of space charge forces is desirable to lower the transverse and longitudinal emittance of the generated electron bunches. A low dark current is necessary to maintain the negative electron affinity properties of the photocathode, thereby increasing the lifetime of the electron source. The most important issue is to reduce field emission within the gun, which is the fundamental source of dark current. The ILC gun R\&D is being carried out at Jefferson Lab and focuses on new materials for the anode and cathode electrodes. New surface-polishing techniques are being investigated and compared. Some examples are traditional diamond-paste polishing, electropolishing and buffer chemical polishing. Promising results have been achieved for chemical-buffered polished niobium electrodes (Figure 3.19).

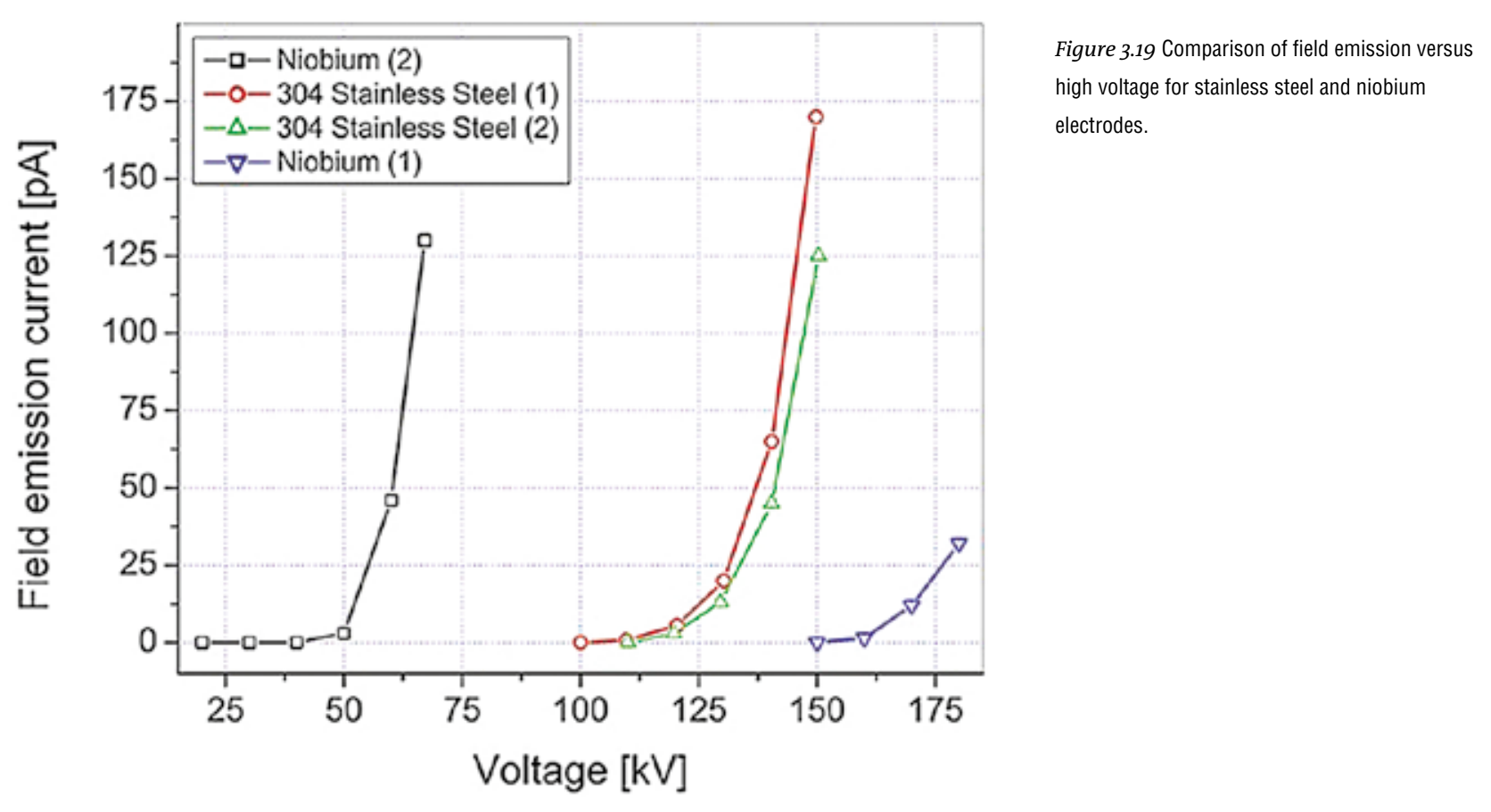


The onset of dark current is significantly higher compared to stainless steel electrodes ( $150 \mathrm{kV}$ vs. $100 \mathrm{kV})$. Additional work focuses on an alternative gun design, the so-called inverted gun, to improve the high-voltage performance. Figure 3.20 shows a niobium electrode for an inverted gun design, which replaces a conventional ceramic insulator with an inverted insulator. This design eliminates the need of sulphur hexafluoride, used in the traditional design to achieve appropriate high-voltage conditions and to ensure no high-voltage breakdown can occur outside the electrode chamber.

Additional emphasis is placed on developing conditioning methods to achieve ultra-high vacuum conditions, which is crucial for successful operation of the gun and improved cathode lifetime.

Figure 3.20 Niobium electrode of inverted gun design.

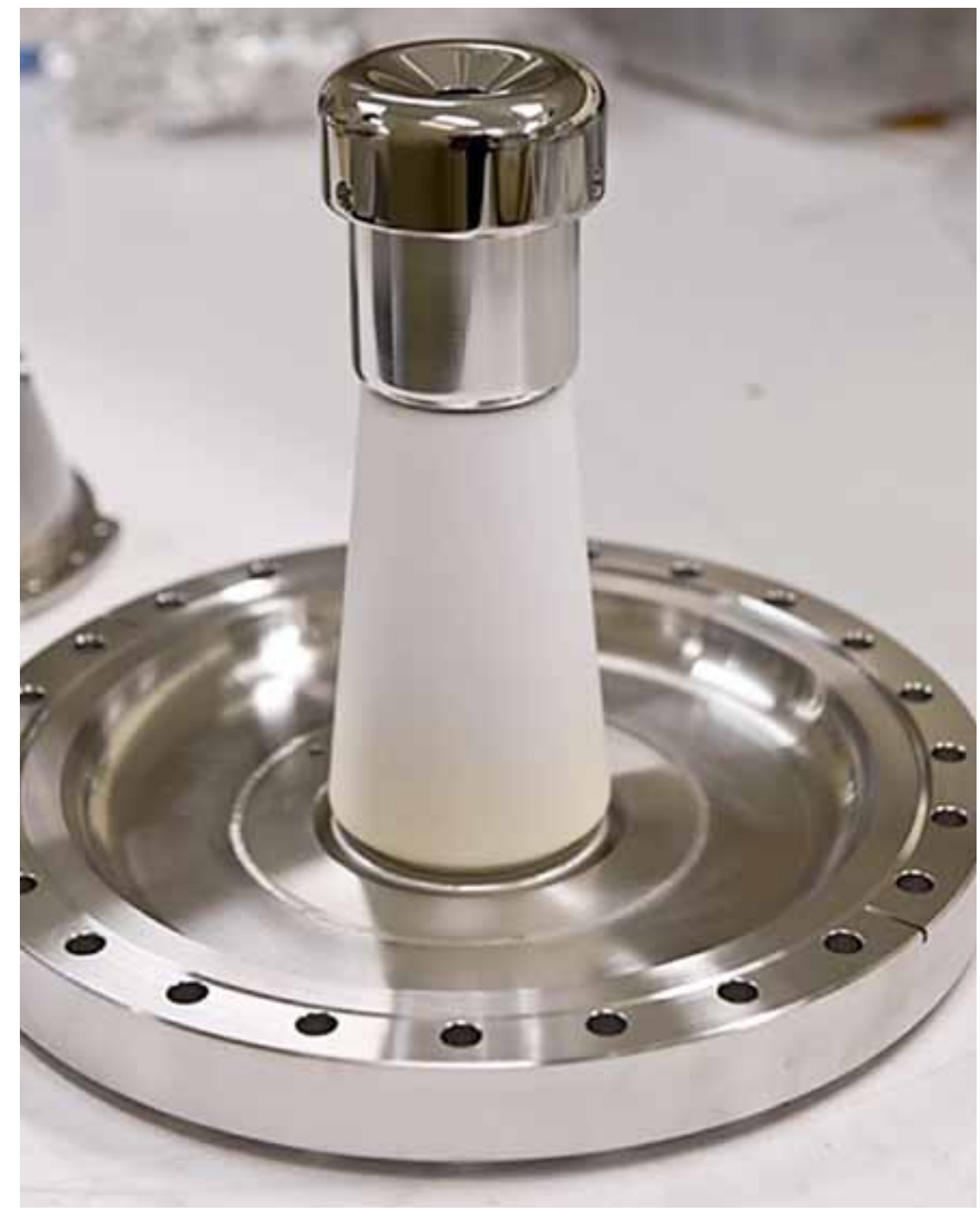




\subsubsection{Positron source}

The ILC baseline for positron production uses the primary high-energy electron beam to generate photons in a long undulator, which subsequently generates electron-positron pairs in a thin target [3-48]. There is a very active $R \& D$ programme associated with the positron source. This system is challenging and novel. As the R\&D has progressed, the design and performance of the source has increased substantially, with many legitimate concerns being dealt with in a systematic manner. Whilst progress has been made in virtually all subsystems, the three key areas of the helical undulator, the conversion target and the flux concentrator have rightly received the most attention as these were previously singled out as higher-risk areas.

\section{Helical undulator}

At the time of the Reference Design Report (RDR), short superconducting helical-undulator prototypes using niobium-titanium superconductors had been successfully fabricated and tested by groups at Rutherford Appleton Laboratory (RAL) in the UK and at Cornell University [3-49, 50] in the US. This gave confidence that the undulator period and field strength selected for the ILC were feasible. Since that time the RAL group has successfully fabricated two identical long undulators, each $1.75 \mathrm{~m}$ in length, which have been magnetically tested and proven easily to achieve the field strength required. In fact, both exceeded the magnetic field specification by more than $30 \%$ [3-51]. The quench training for the two magnets is shown in Figure 3.21.

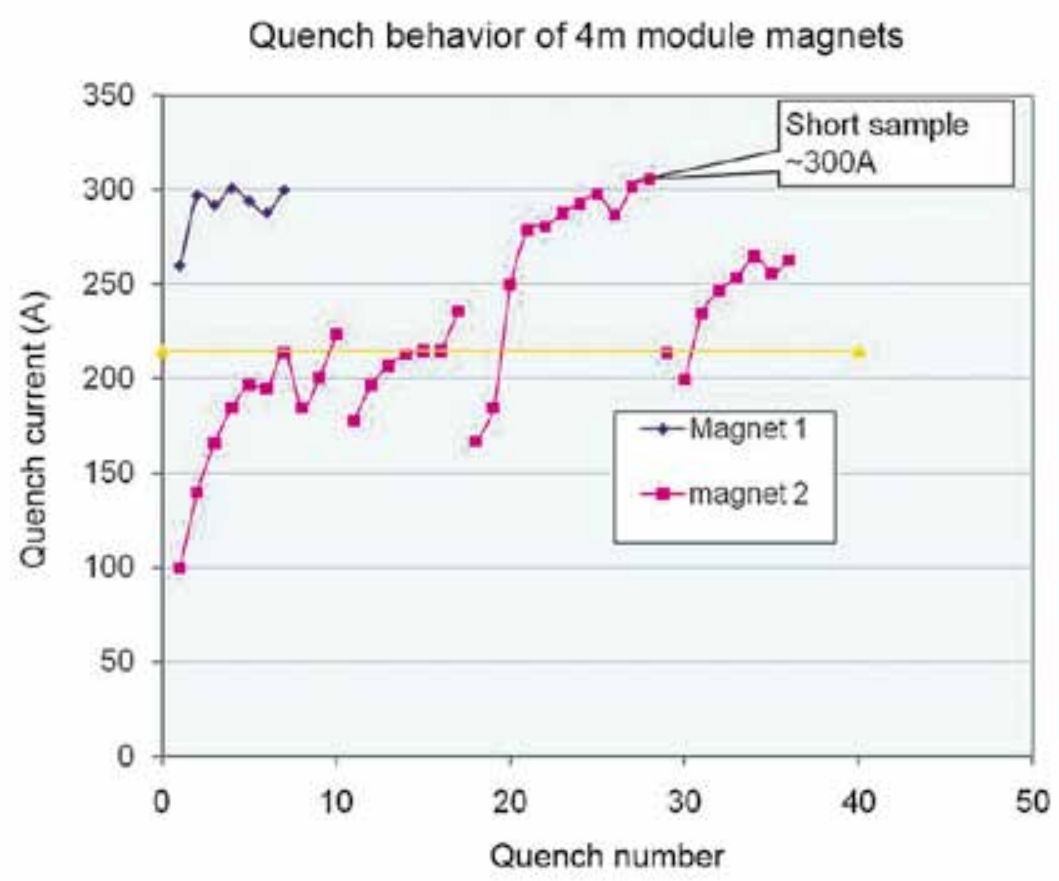

Figure 3.21 Quench history of the 3-m prototype superconducting helical undulator. 
Figure 3.22 The 4-m prototype superconducting helical undulator under test at Rutherford Appleton Laboratory.
In addition the subsequent analysis of the magnetic field results by staff at Daresbury Laboratory in the UK has shown that both undulators have a very high field quality, certainly more than sufficient to provide the intense source of gamma photons that is required. The RAL team has since incorporated both of these undulators into a single 4-m-long cryogenic module (which operates at $-269^{\circ} \mathrm{C}$ ) of the design required by the ILC, and has proven that both undulators can be powered simultaneously at the field levels required [3-52]. A photo of the complete undulator cryomodule is shown in Figure 3.22. In the future it would be valuable to install the module into an electron beam test line to measure the photon properties of the light generated by the undulators.

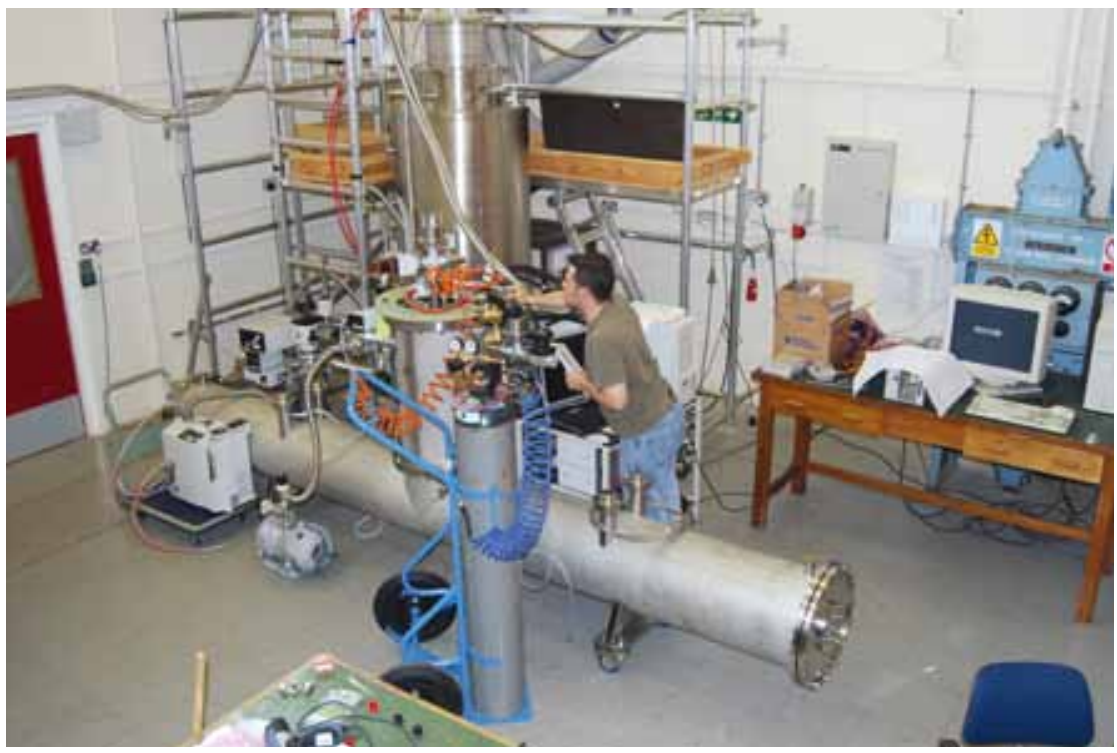

The RAL team is now investigating the use of a more advanced superconducting material, niobium tin, which should enable even higher field strengths to be generated. If this is proven to be the case in practice it will enable the period of the undulator to be reduced further, which will allow the positron source to generate the required positron yield at lower electron drive-beam energies, a considerable advantage to the ILC project. Currently the team is winding short prototypes to gain experience with this technically more challenging material and also to allow a direct comparison with the other prototypes built using niobium titanium [3-53].

\section{Conversion target}

The conversion target is a 1-m-diameter wheel of titanium alloy that rotates at $100 \mathrm{~m} / \mathrm{s}$ at the rim. To increase the positron yield, the target rim passes through a strong magnetic field. Unfortunately, this then induces unwanted eddy currents in the wheel, causing the wheel to heat up. The level of heating that can be tolerated limits the usable magnetic field. Several groups have tried to model the eddy current heating but inconsistent results were obtained from the different simulation codes they used [3-54, 55]. Consequently a full-scale prototype target has been built at the Cockcroft 
Institute in the UK to benchmark the simulation codes. A full-size target wheel was fabricated from the required titanium alloy and was rotated over a range of rim velocities in a strong magnetic field (Figure 3.23). The results of this unique experiment have accurately quantified the eddy current effects and have confirmed which simulations were correct [3-56]. Furthermore, the experiment has proven that the magnetic field level assumed by the positron source design at the target wheel is feasible, with the eddy current heating being easily tolerated.

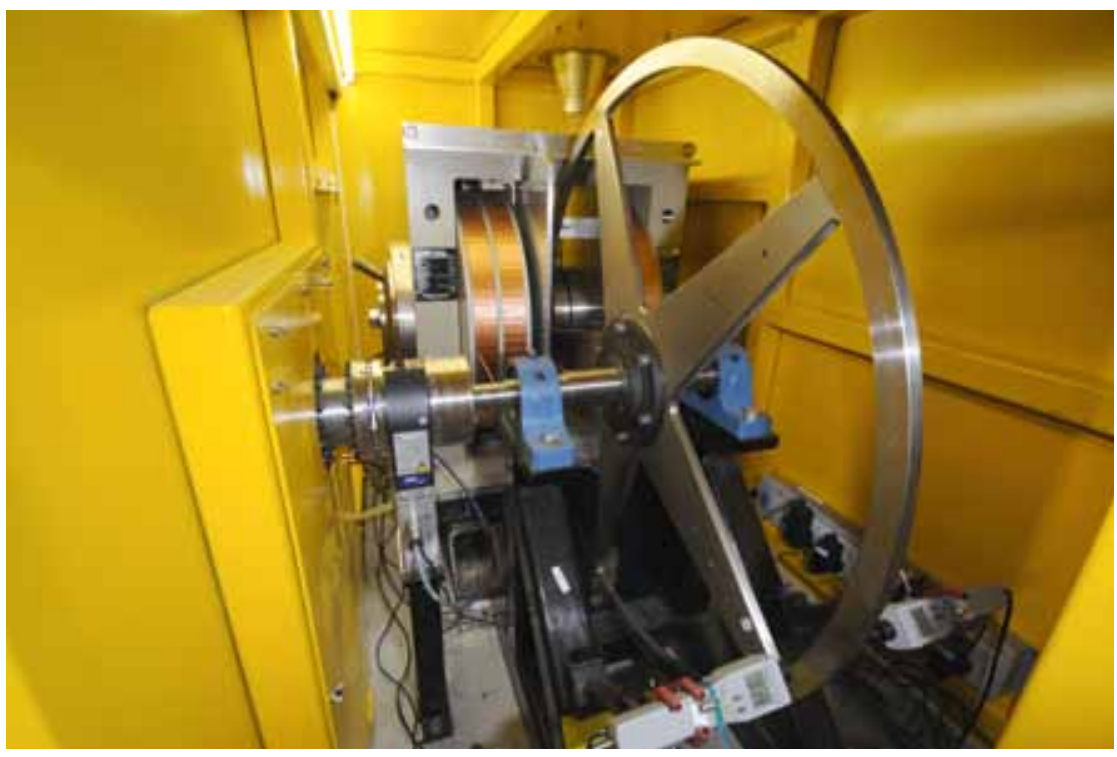

Figure 3.23 Prototype rotating target setup for eddy current tests at Daresbury Lab. Copyright STFC.

The target wheel also has to operate inside a vacuum chamber whilst the motor is in air. This means that a rotating vacuum seal is required that is capable of operating at high velocity, near a magnet and in a high radiation environment - quite a demanding challenge. The team has identified a commercial vacuum seal that, the manufacturers claim, is suitable for ILC conditions. To confirm the long-term performance of the seal, a relatively simple test is currently being planned by staff at Lawrence Livermore National Laboratory (LLNL) in the US. Initially, an equivalent load to the target will be rotated in a vacuum and the performance of the seal evaluated by monitoring the vacuum level within the chamber. Later the full-size target wheel that is currently at the Cockcroft Institute will be delivered to LLNL, and be rotated at the speeds required by the ILC under vacuum. The engineering design concept for this test is shown in Figure 3.24. 
Figure 3.24 Schematic of the engineering design for the in-vacuum rotating target.

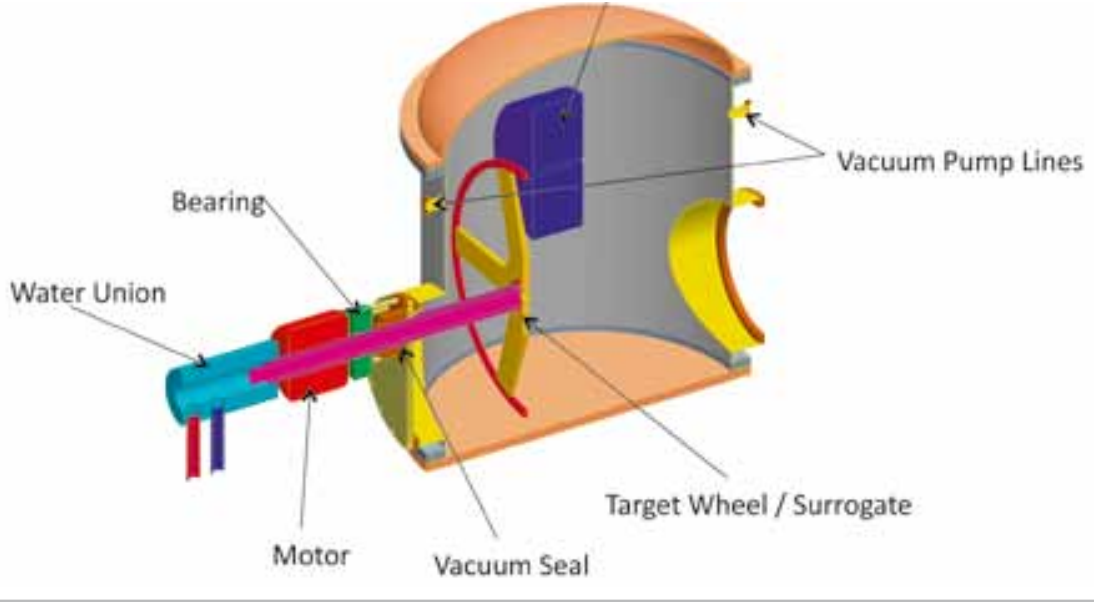

Another issue for the target that has been studied in detail is the effect of the shockwave on the target as a consequence of being struck by the intense pulses of gamma photons generated by the undulator [3-56]. Concerns were raised over possible material damage to the target itself on a shot-by-shot basis. Simulations with a numerical code at LLNL suggested that the effect is not significant. This has since been confirmed with a detailed analytical study, carried out at Durham University in the UK [3-57].

\section{Flux concentrator}

The flux concentrator is the pulsed magnet that generates the strong magnetic field close to the target wheel in order to enhance the positron yield. Many of these have been used successfully in the past but the parameters of the ILC require a more technically challenging device. A detailed R\&D study has been initiated at LLNL to confirm the feasibility of the proposed magnet and later to build a suitable prototype to demonstrate the design performance. The team has shown that the flux concentrator must be operated at around $-200^{\circ} \mathrm{C}$ using a liquid-nitrogen cooling system so that the electrical conductivity of the very high current-carrying copper disks do not generate too much resistive heating. The design is now well advanced (see Figure 3.25) and the simulations predict excellent performance of the magnet [3-58]. The next step is a phased prototyping of the magnet to demonstrate the key features of the design. The first tests will be carried out at room temperature and at low repetition rates. These will confirm the magnetic field strength and profile is as required. The next step will be to cool the magnet with liquid nitrogen and to confirm reliable operation at 5 $\mathrm{Hz}$ over an extended period. 


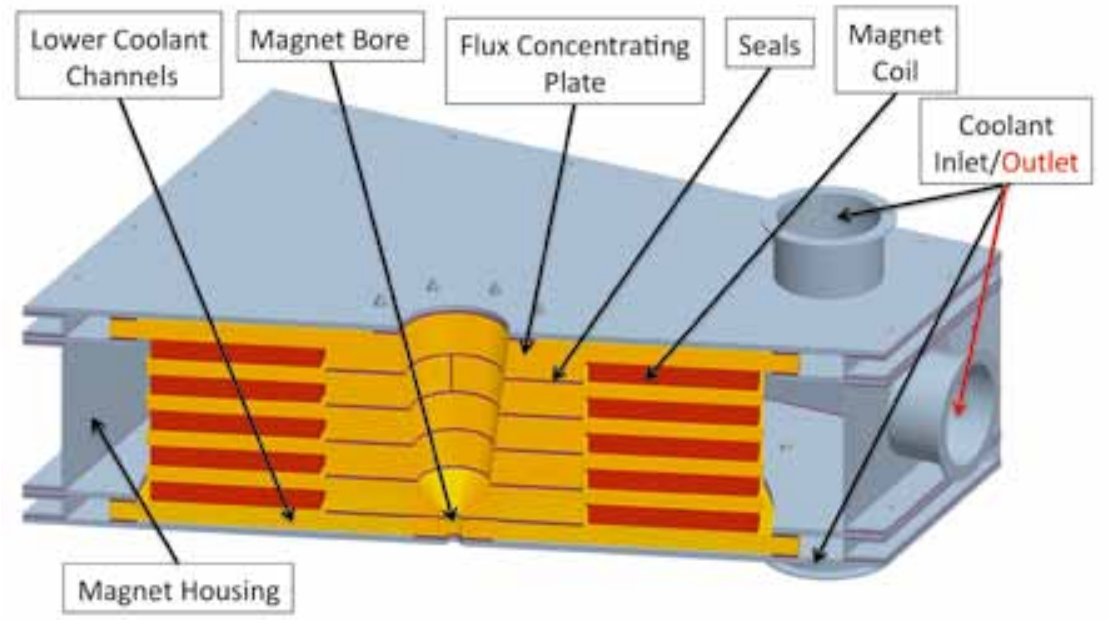

Figure 3.25 Section of the prototype design for the pulsed flux concentrator.

\section{Performance simulations}

The parameters for source subsystems and the determination of the source performance can only be quantified using complex simulations. These simulations quite often require the combination of several sophisticated computer codes. The primary performance figure of merit is the yield, defined as the number of positrons captured in the acceptance of the damping ring per electron passing through the undulator. (Ideally a yield of 1 is required, but the design goal is set at 1.5 to allow a $50 \%$ safety factor.) The second figure of merit is the polarisation of the captured positrons, which depends on additional parameters such as the collimation aperture of the photon beam before the target. Figure 3.26 shows an example of integrated performance simulation [3-59].

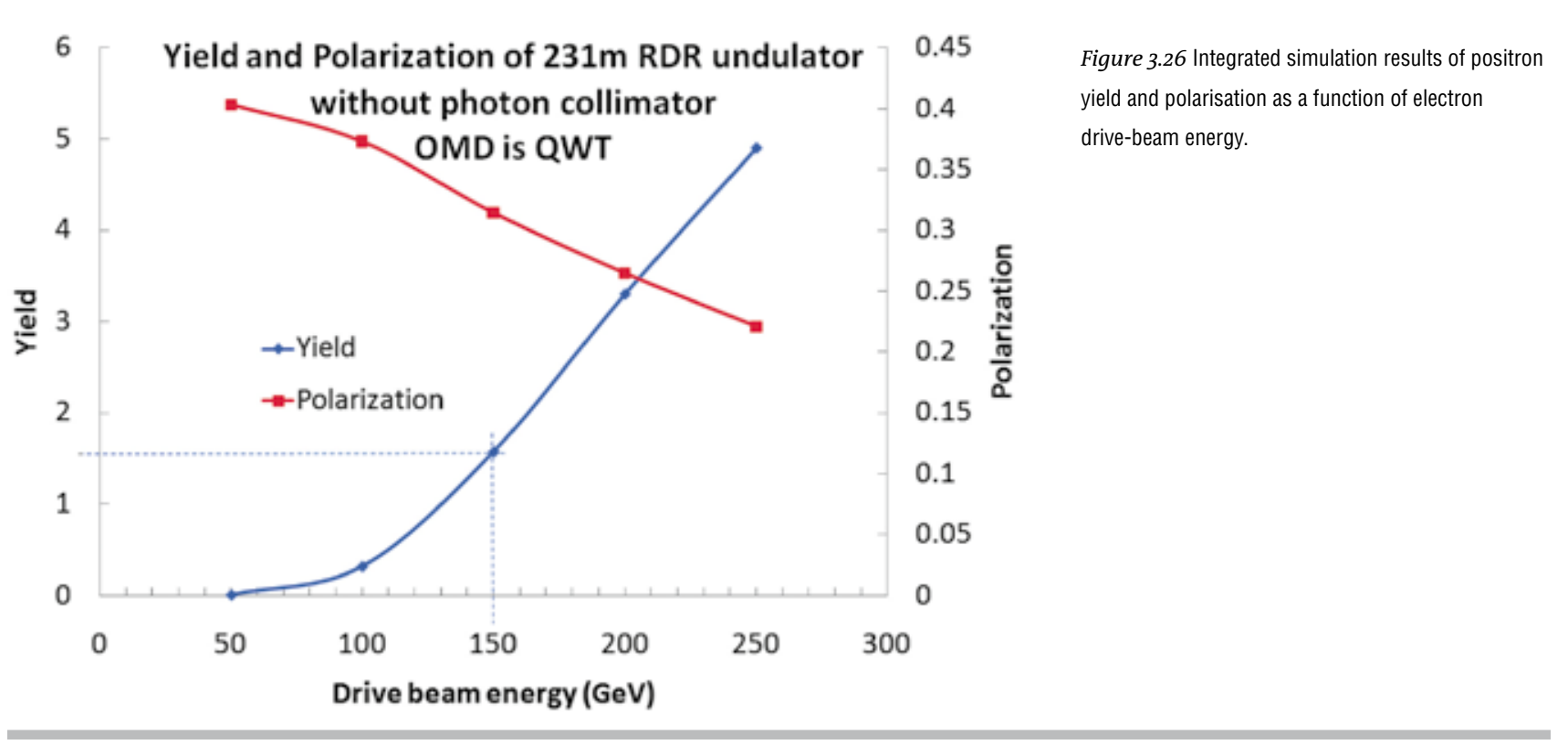


Figure 3.27 Observed positron yield as a function of the incident beam angle with respect to the crystal axis. An enhancement of a factor of approximately 3 is seen at the correct orientation.

\section{Alternative sources}

Since the undulator-based source represents a novel concept, more conventional options using an electron beam on a thick target are also being investigated as possible backup solutions. In the first such option, the positron beam is produced by a few-GeV electron beam from a normalconducting linac with a beam pulse length of approximately $1 \mu$ s and a repetition rate of around $300 \mathrm{~Hz}$. The total 2,630 positron bunches required are produced in $60 \mathrm{~ms}$ (as opposed to a single 1-ms pulse for the undulator source) to reduce the peak target heat load. In this case the flux concentrator technology is less demanding owing to the shorter $(1 \mu \mathrm{s})$ pulse length. As a second option, a system with multiple targets could be used, but a more elegant solution would be a single liquid-lead target system, a prototype of which is currently being tested at KEK-ATF [3-6o]. A third possible electrondriven scheme is the 'hybrid-target system' in which a photon beam is produced from a few-GeV electron beam at an enhanced rate by using a crystal target. The charged particles produced by the crystal target are swept out by a magnetic field, leaving only the photon beam to irradiate a second amorphous target to produce positrons. A basic experiment is being done at KEK using the KEK-B linac [3-61] (see Figure 3.27).

The common disadvantage of electron-driven sources is that the positron beam is unpolarised. As a possible future advanced scheme for polarised positron production, methods using laser Compton scattering are also under study [3-62].

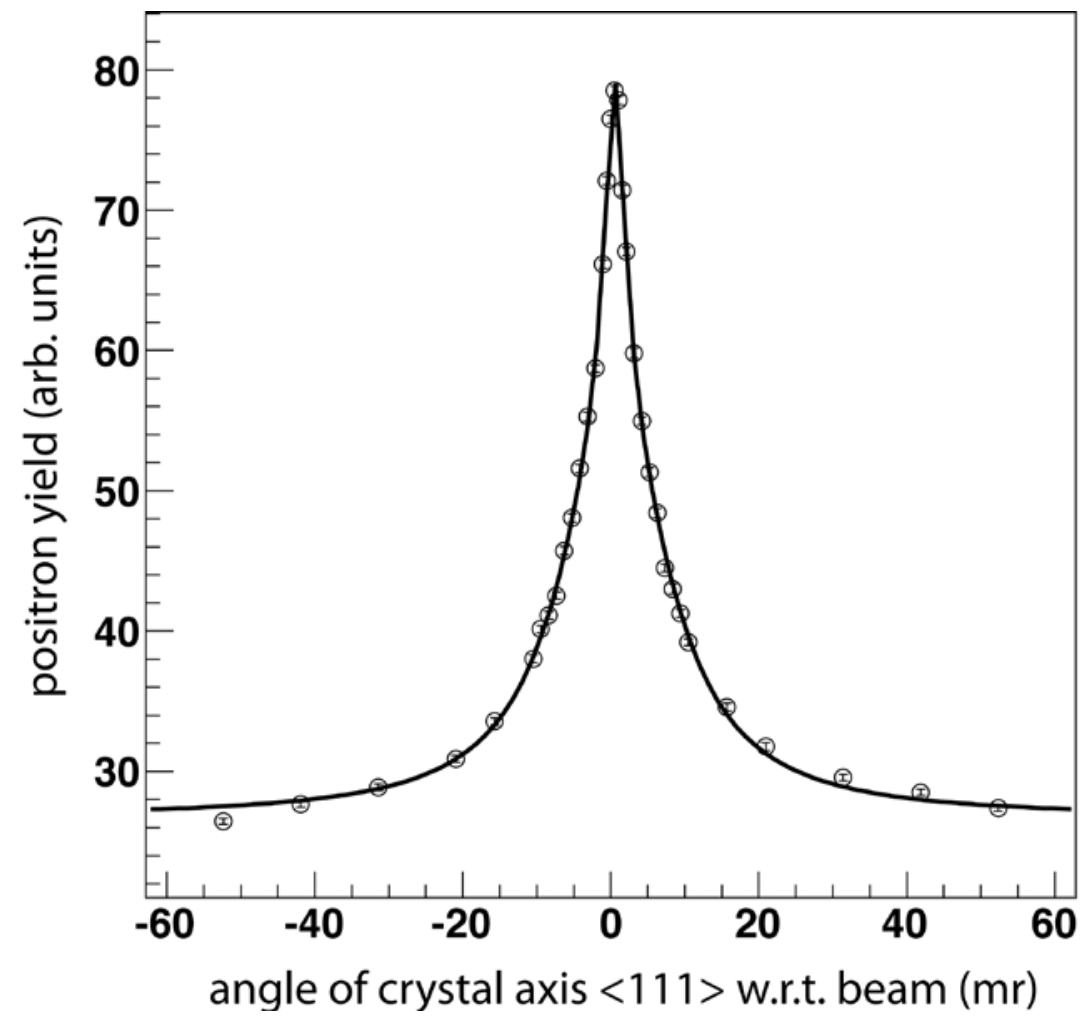




\subsubsection{Damping ring}

The ILC R\&D programme identified the key areas for work during the

Technical Design Phase:

1. developing methods to suppress the electron cloud instability

2. demonstration of ultra-low vertical emittance operation (vertical emittance of $2 \mathrm{pm}$ )

3. demonstration of fast injection/extraction kickers performance.

Two dedicated test facilities were identified for this effort: CesrTA at Cornell University and ATF at KEK. Both programmes have managed large collaborations, with contributors from institutions worldwide working on simulation, experiment and design.

\section{Electron cloud mitigation}

The R\&D effort on electron cloud mitigation involves the large international collaboration gathered around the CesrTA programme plus the effort that is in progress at other laboratories.

This successful damping ring R\&D endeavour is described in more detail in section 3.1 on the CesrTA programme.

\section{Ultra-low emittance operation}

The demonstration of ultra-low emittance was carried out in the framework of the CesrTA and ATF collaborations, but important results have also come from the synchrotron light sources community.

\section{DiAGNOSTICS FOR LOW-EMITTANCE BEAMS AT ATF}

The ATF damping ring achieved a vertical emittance as low as $4 \mathrm{pm}$ before the publication of the RDR and has supported a wide range of important research for many years: low-emittance tuning and intrabeam scattering studies, studies of the fast ion effect and fast kicker tests. Now the damping ring's main focus is the production of an extracted beam with the required characteristics for the ATF2 programme (see section 3.2) and the development and test of low-emittance beam diagnostics. Instrumentation development includes laser wire, optical transition radiation, optical diffraction radiation, and a high-resolution X-ray monitor [3-63].

\section{DiAgnostics AND TUNING ALGORITHMS AT CESRTA}

The low-emittance tuning effort provides the foundation for studies of the emittance-diluting effects of the electron cloud in a regime approaching that of the ILC damping rings. The vertical emittance goal for the initial phase of the CesrTA programme is less than $20 \mathrm{pm}$. Low-emittance tuning efforts have focused on the systematic elimination of optical and alignment errors that are the sources of vertical emittance degradation [3-64]. Techniques have been developed to eliminate beam position monitor systematic errors, measuring gain variation among the four button-electrodes on each beam position monitor, and to centre the monitors with respect to the adjacent quadrupole. Work has also been carried out to optimise the sextupole design, thus minimising sources of emittance coupling. During the most recent experimental run, this effort resulted in measurements of the vertical emittance consistent with having achieved the target vertical emittance of $20 \mathrm{pm}$ in both single-bunch and multi-bunch operations. 
An X-ray beam size monitor has been developed and successfully demonstrated at CesrTA. It is able to measure both integrated and singlebunch turn-by-turn beam sizes at positions for monitoring the progress of the low-emittance tuning of the machine and for beam dynamics related to instabilities driven by the electron-cloud [3-65, 66].

\section{DEMONSTRATION OF VERTICAL EMITTANCE BELOW 2 PM}

\section{AT SYNCHROTRON LIGHT SOURCES}

A step forward in the demonstration of very low vertical emittance has been achieved at some synchrotron light sources, where they operate low-emittance storage rings with characteristics very similar to the ILC damping ring and have developed alignment procedures, machine modelling, tuning algorithms, and orbit stabilisation for coupling correction and low vertical emittance tuning [3-67]. In particular, the Diamond Light Source in the UK, the Swiss Light Source and the Australian Synchrotron storage ring have achieved betatron coupling correction down to $0.1 \%$ and vertical emittances below $2 \mathrm{pm}[3-68,69,70]$. Significant progress has been made in the development of diagnostic systems for the measurement of such small vertical emittances [3-71, 72, 73]. The Low Emittance Ring workshop, held at CERN in January 2010 and organised by the joint ILC-CLIC working group on damping rings, was very successful in strengthening the collaboration within the two damping ring design teams and with the rest of the low-emittance rings community, including synchrotron light sources and B-factories.

\section{Performance of fast injection/extraction kickers}

\section{ILC-LIKE MULTI-BUNCH EXTRACTION AT ATF}

The injection/extraction kickers act as the bunch-by-bunch beam manipulator to compress and decompress the bunch spacing into and from the damping ring. The kickers require high repetition frequency, 3 (or 6) $\mathrm{MHz}$, and very fast rise and fall times of the kicker field: $6 \mathrm{~ns}$ for the nominal configuration and $3 \mathrm{~ns}$ for a proposed luminosity upgrade. The tolerance on horizontal beam jitter of the extracted beam is approximately $10 \%$ of the beam size, which requires the extraction kicker amplitude relative stability to be below $7 \times 10^{-4}$.

A rise and fall time of 3 ns has been already demonstrated in the ATF using a 30-cm-long strip-line kicker together with a semiconductor high-voltage pulse source [3-74]. The time response of the strip-line kicker was observed by measuring the resulting betatron oscillation amplitude of the stored electron beam.

An ILC-type beam extraction experiment using two strip-line kickers has been carried out at KEK-ATF [3-75]. The length of the strip-lines is $60 \mathrm{~cm}$ and the gaps of the two electrodes are $9 \mathrm{~mm}$ and $11 \mathrm{~mm}$. Two pairs of pulsers with a peak amplitude of $10 \mathrm{kV}$, a rise time of $1.5 \mathrm{~ns}$ and a repetition rate of 3.3 $\mathrm{MHz}$ are used to drive the strip-lines. The strip-line kicker system produced a 3-mrad total kick angle for the $1.3-\mathrm{GeV}$ beam. The rise time of the kick field is less than $5 \mathrm{~ns}$. 
The multi-bunch beam stored in the damping ring with 5.6-ns bunch distance was successfully extracted with 308-ns bunch spacing in the extraction line (Figure 3.28). No deterioration of the extracted vertical beam size was observed (as measured with the laser wire). The resynchronisation circuit used for precise timing adjustment worked stably. The relative angle jitter of the single bunch beam extraction was $3.5 \times 10^{-4} \mathrm{rms}$, which is better than the requirements for ILC damping ring extraction. For multi-bunch beam extraction a trigger timing circuit is needed to compensate the time drift of the pulser. Very recently, 30-bunch extraction with an rms angle jitter about $10^{-3}$ has been achieved. This value can be further reduced by precisely tuning of the timing system or by using a feed-forward system.
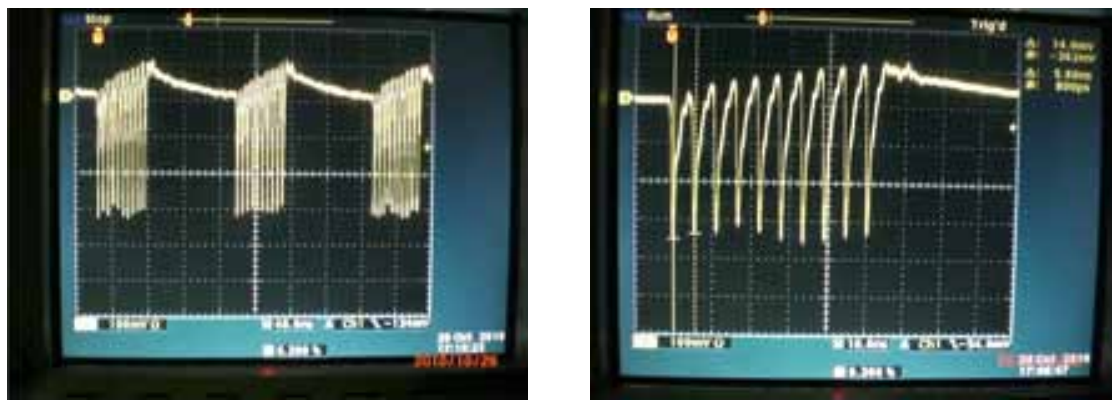

Figure 3.28 ATF damping ring. Top: three trains of ten bunches with 5-ns spacing (right image is a close-up of waveforms seen in the left image). Bottom: 30 bunches extracted with 308-ns spacing.

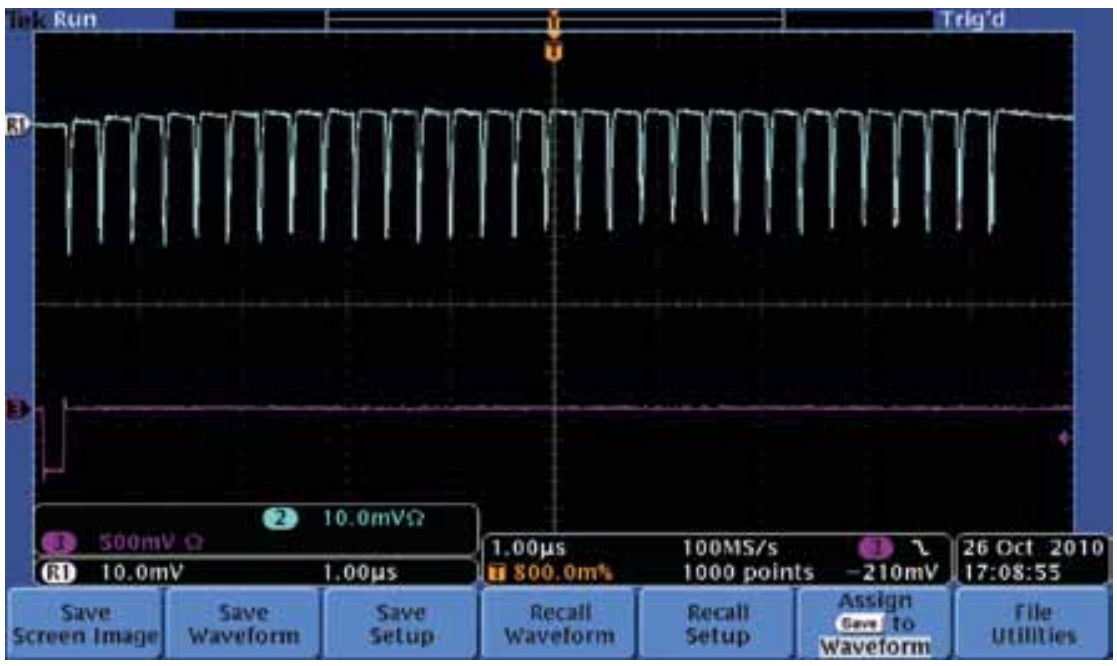

\section{STRIP-LINE KICKER DESIGN AT DAФNE}

The design of the new, fast strip-line kickers for the injection upgrade of the DAФNE $\Phi$-factory is based on strip-line tapering to obtain a low-beam impedance device and an excellent uniformity of the deflecting field in the transverse plane (Figure 3.29) [3-76]. These characteristics are essential also for the ILC damping ring, and the experience gained with the new DAФNE injection system will be applied to the damping ring injection system design. The rise and fall times of the kickers are all less than $6 \mathrm{~ns}$, corresponding to the damping ring requirement for the nominal configuration. 
The coupling impedance measurements and simulations have pointed out the absence of trapped higher-order modes in the longitudinal and horizontal planes when at least two ports are loaded by 50 watts [3-77]. In the vertical plane only four trapped higher-order modes were found. The instability growth rates of these resonances (in the worst case) were well below the damping rates provided by the DAФNE feedback systems. After installing the injection system, no instability effects due to the kickers were observed and the DAФNE broadband impedance arising from this and other vacuum chamber modifications made at the same time was reduced by about $50 \%[3-78]$.

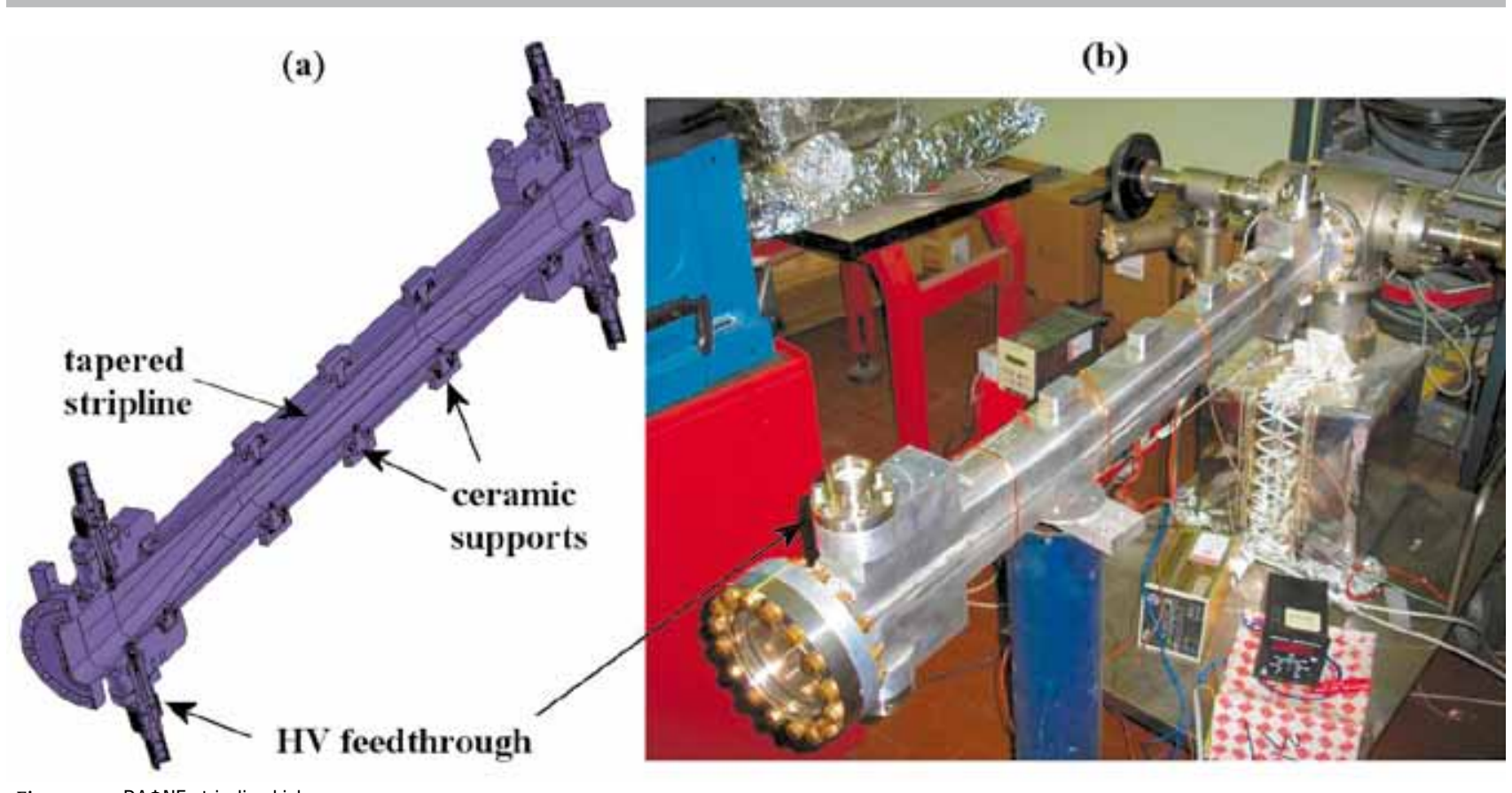

Figure 3.29 DAФNE strip-line kicker.

\section{SLAC PULSER MODULATOR}

At SLAC, two related paths to meet the ILC kicker driver requirements are being studied: a transmission line adder topology, which combines the output of an array of ultra-fast MOSFET (metal-oxide-semiconductor fieldeffect transistor) switches and a drift step recovery diode (DSRD) approach.

For the adder topology, an ultra-fast hybrid MOSFET/driver, recently developed at SLAC, has achieved 1.2-ns switching of 33 amperes at 1,00o volts with a single power MOSFET die [3-79]. A transmission-line adder has been designed based on the ultra-fast hybrid MOSFET/driver switching module. The initial test demonstrated that the adder can combine pulses with 1.4-ns switching time without any degradation [3-80]. The programme continues to extend the system to the ILC parameters. 


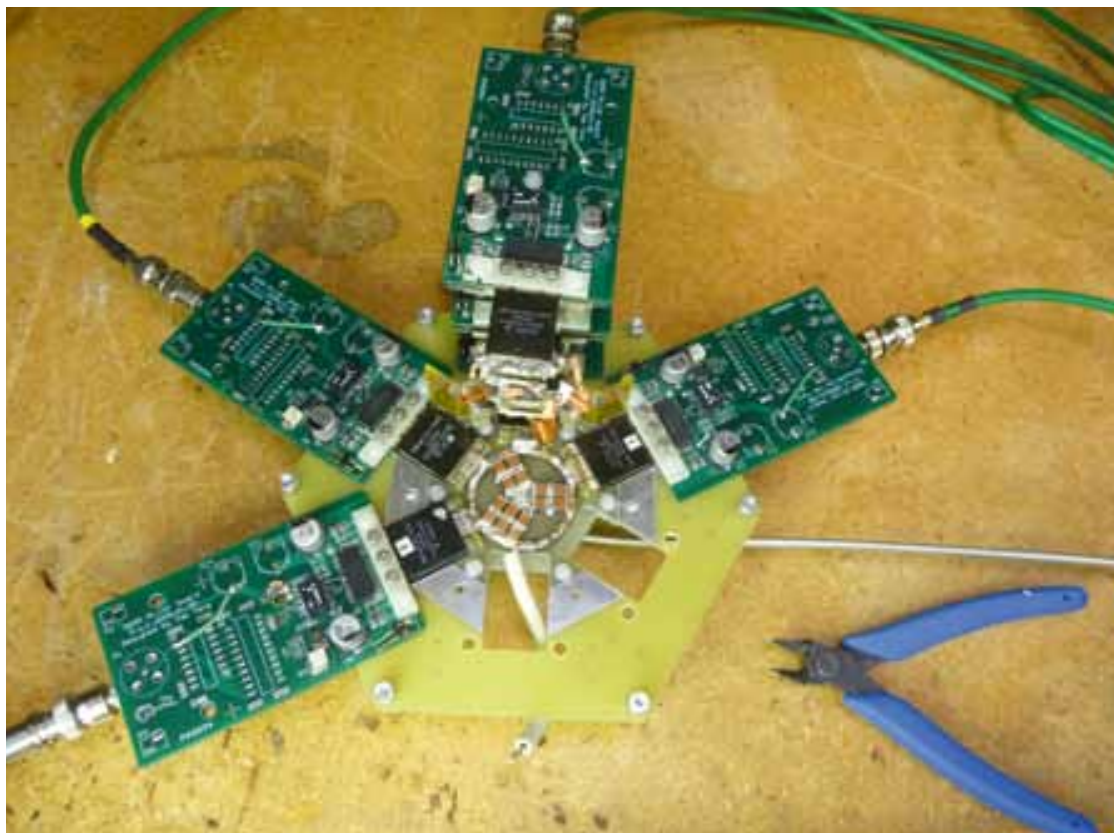

Figure 3.30 A partially assembled drift step recovery diode circuit of a SLAC kicker modulator.

For the DSRD programme, development of a fully capable DSRD kicker driver is proceeding well, with excellent results obtained from the first commercially produced DSRDs, and from a refined circuit for the MOSFET driver [3-81]. A prototype with 2-ns pulse length and 1-MHz pulse train has been demonstrated (Figure 3.30). A recent success was to eliminate the post-pulse, which is unacceptable for the ILC kicker driver since it affects the bunches adjacent to the kicked bunch [3-82]. The plan is now to build a demonstration modulator for beam testing at ATF.

\subsubsection{Beam delivery system}

\section{Final doublet design and prototyping}

The interaction region magnets are one of the most challenging systems. Design and prototyping of the final-doublet magnets is proceeding and is illustrated in Figures 3.31 and 3.32. One particular difference of the present final doublet design with respect to the RDR version is that the last 2-m-long coil defocusing quadrupole (QDo) is split into two separate coils, which allows introduction of a mechanical support point in the middle of the quadrupole cold mass. This modification was found to be necessary during prototyping of the long coil for the final doublet, and will also benefit a proposed modular approach for optimising the optics for low-energy running (shorter QDo). Cryostats have been designed to house the magnets, providing the necessary stable support of the magnet, while being compatible with the requirements of the push-pull arrangement of the detectors. 
Figure 3.31 Final doublet magnet design and prototype of the long coil.

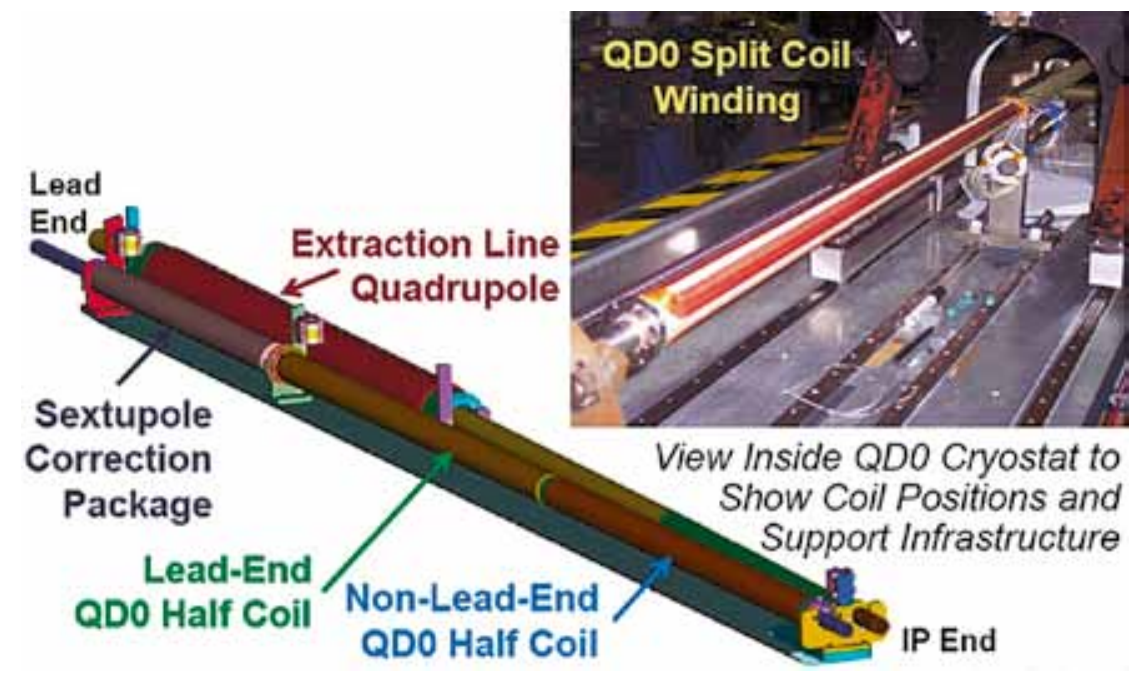

Figure 3.32 Details of design of the final doublet cryostat.

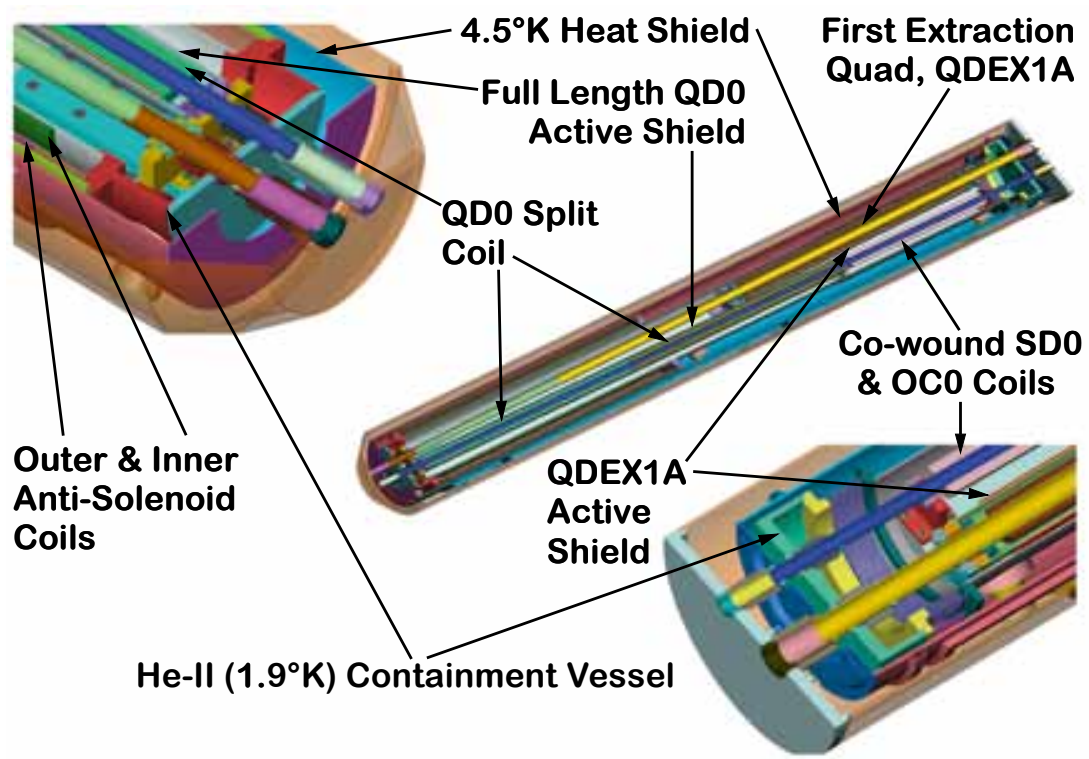

\section{MACHINE-DETECTOR INTERFACE}

The push-pull system for the two detectors was only conceptual at the time of the publication of the RDR, and since then the engineering design has progressed significantly. A time-efficient implementation of the push-pull model of operation sets specific requirements and challenges for many detector and machine systems, in particular the interaction region magnets, the cryogenics, the alignment system, the beamline shielding, the detector design and their overall integration. The minimal functional requirements and interface specifications for the push-pull interaction region have been successfully developed and published [3-83], to which all further related design work on both the detectors and machine sides are constrained. 
The push-pull design needs to accommodate the two detector concepts, the International Large Detector (ILD) and the Silicon Detector (SiD), which are different in their designs, dimensions and mechanical characteristics (such as mechanical rigidity). The different sizes provide particular challenges for the beamline shielding elements, collectively referred to as the pacman shielding. An example of a design of the pacman shielding that ensures compatibility with both detectors is illustrated in Figure 3.33.

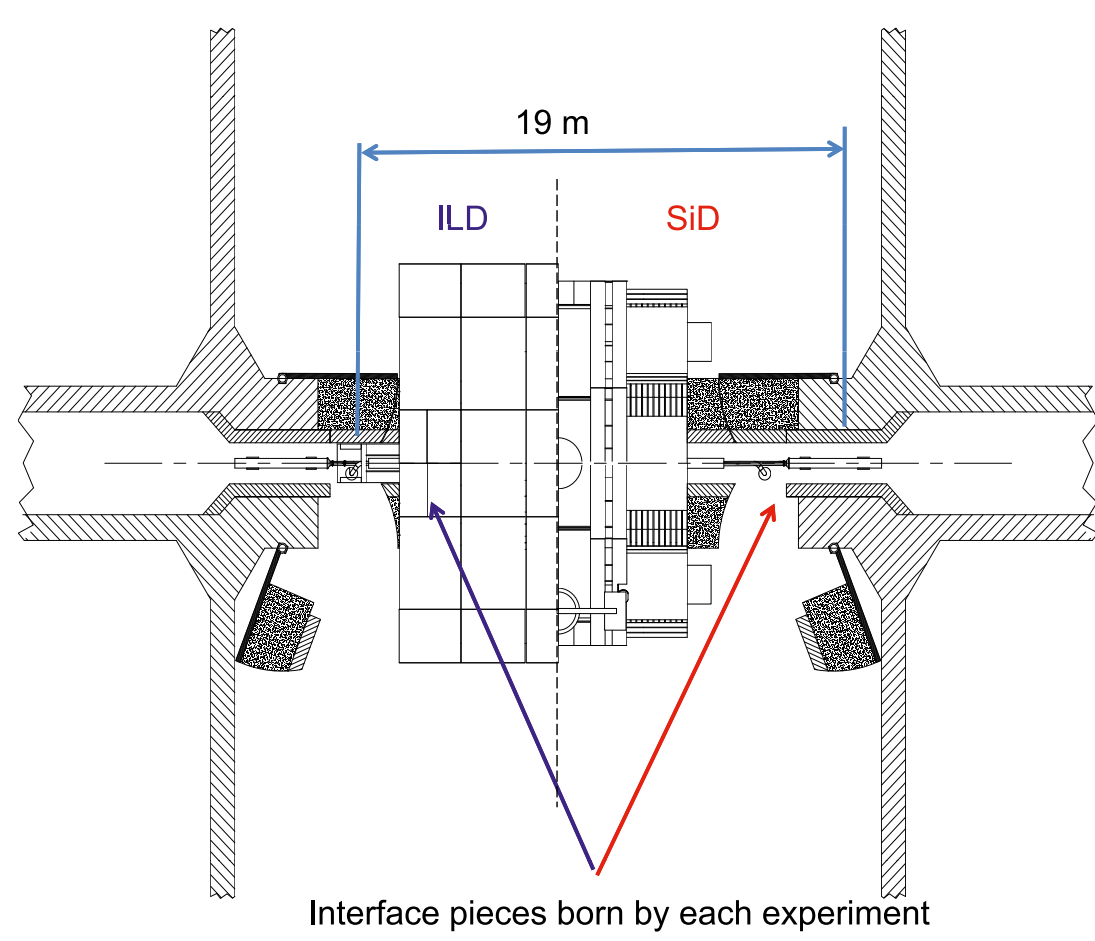

Figure 3.33 Design of the beamline shielding compatible with two detectors of different sizes.

The detector motion and support system has to be designed to ensure reliable push-pull operation, allowing a hundred moves over the lifetime of the experiment while preserving internal alignment of the detector's internal components and ensuring accuracy of detector positioning. The motion system must be designed to preserve structural integrity of the collider hall floor and walls. Moreover, the motion and support system must be compatible with the tens-of-nanometre-level vibration stability of the detector. If the collider is built in a moderate or high seismic region, the system must also be compatible with earthquake safety standards. Two different approaches for the detector support system are currently being considered. The ILD detector is somewhat larger than $\mathrm{SiD}$ and is also designed to be assembled from slices in a way similar to the LHC Compact Muon Solenoid detector. The ILD motion system will thus benefit greatly from the use of a rigid platform on which the entire detector can be placed. The platform will preserve detector alignment and will distribute the load evenly onto the floor. Such an approach is illustrated in Figure 3.34. The more compact and rigid SiD can be supported naturally by an eight-leg structure as shown on Figure 3.35. 
Figure 3.34 Possible platform support concept for the ILD. Left: detector is positioned on the beamline. Right: detector is off the beamline.
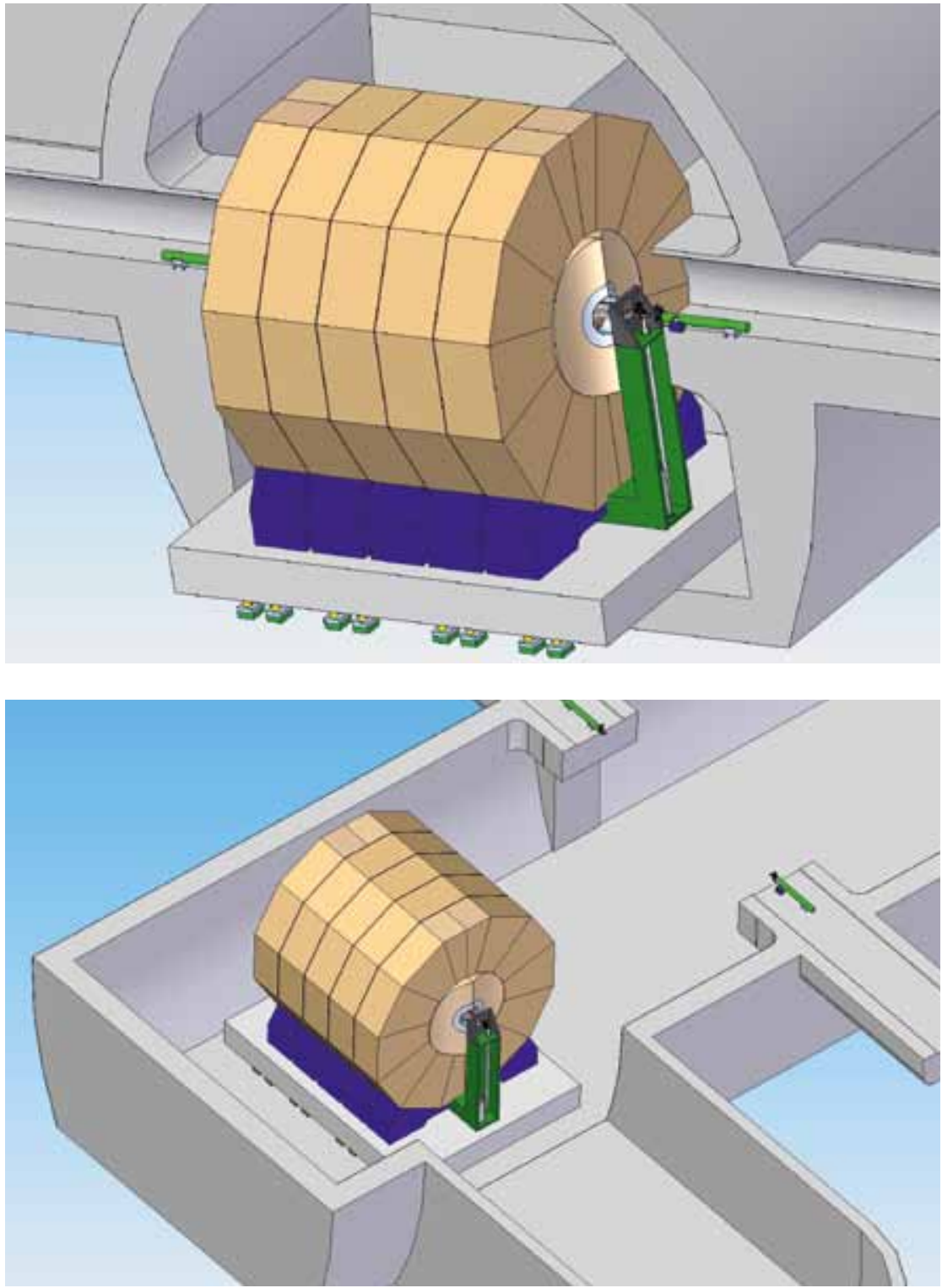

The approach for the design of the detector motion system and in particular the use of a platform is currently being investigated. The criteria for selection of the common design will be based on vibration stability analysis of the entire system (detector together with its support and motion system). The selection is planned to happen in the near future. 


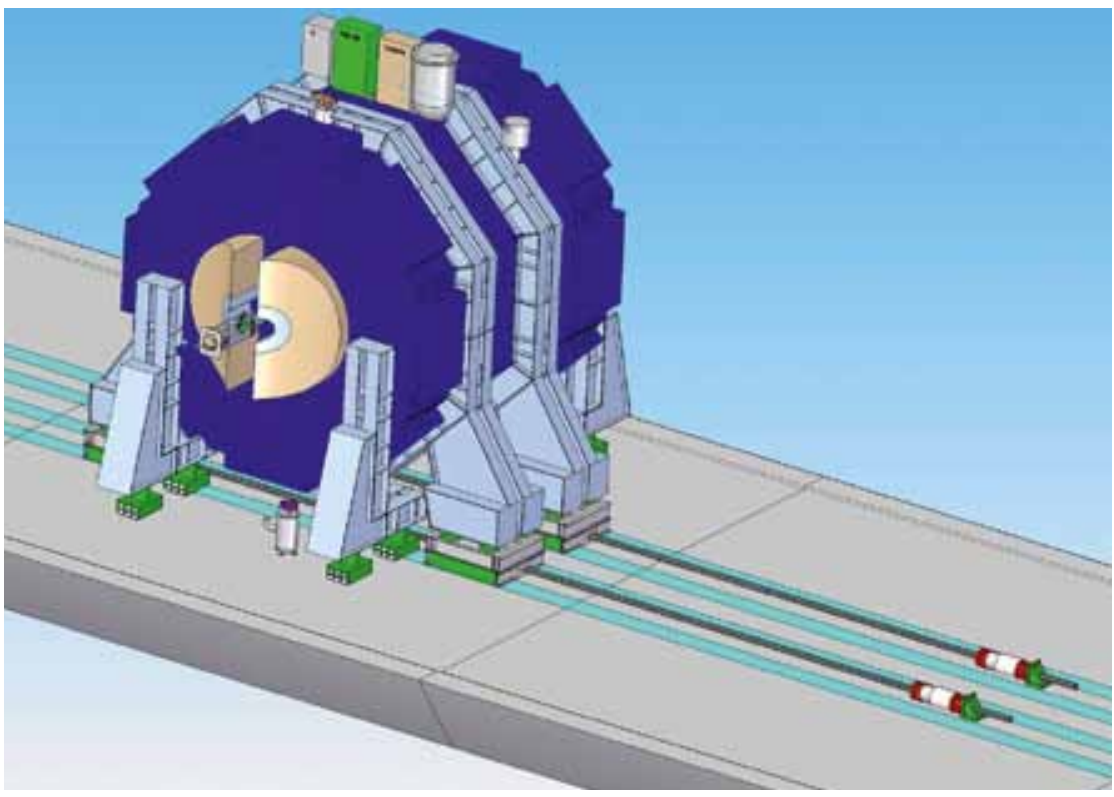

Figure 3.35 Possible detector motion system for SiD.

HIGH-POWER BEAM DUMP SYSTEMS

High-power beam dumps are essential components of the beam delivery system. The main beam dumps are located at the end of the post-interaction point extraction lines. A further two dumps located further upstream (before the final focus beamlines) are envisaged as tune-up and commissioning dumps. The pressurised water dumps must be capable of dissipating up to 18 megawatts (MW) of average beam power (to accommodate the energy upgrade to $1 \mathrm{TeV}$ centre-of-mass).

The dump design for the beam delivery system is based on the 2.2-MW directconvection dump developed and successfully operated for the Stanford Linear Accelerator. Water is injected azimuthally with an appropriate velocity near the outer periphery through a water header located parallel to the beam propagation, which gives rise to a vortex flow. The water is collected in a header located along the axis of beam dump. The beam enters the dump off-axis at a location of high-vortex flow. The length of the beam dump is determined by the energy deposited by the beam in the end plate of the dump. The design parameters are chosen to prevent a temperature rise of the water that would result in boiling. A further critical design item is the beam dump window, which needs to be thin enough to survive the beam passage while supporting the water pressure in the dump. The detailed design of the complete beam dump system, including window and cooling system, is currently being developed [3-84]. The work includes detailed hydrodynamic simulations of the dump, of which an example is given in Figure 3.36. The approximately $1-\mathrm{ms}$ beam pulse is assumed to be rastered by a system of magnets upstream of the dump, which smears the energy distribution in the water and in particular over the millimetre-thin window. 
Figure 3.36 Temperature distribution at the shower maximum of the beam in the dump just after passage of the beam train. The colour bar shows temperature in kelvins, with the maximum temperature equal to $155^{\circ} \mathrm{C}$. The water inlets and sink are shown by white areas.
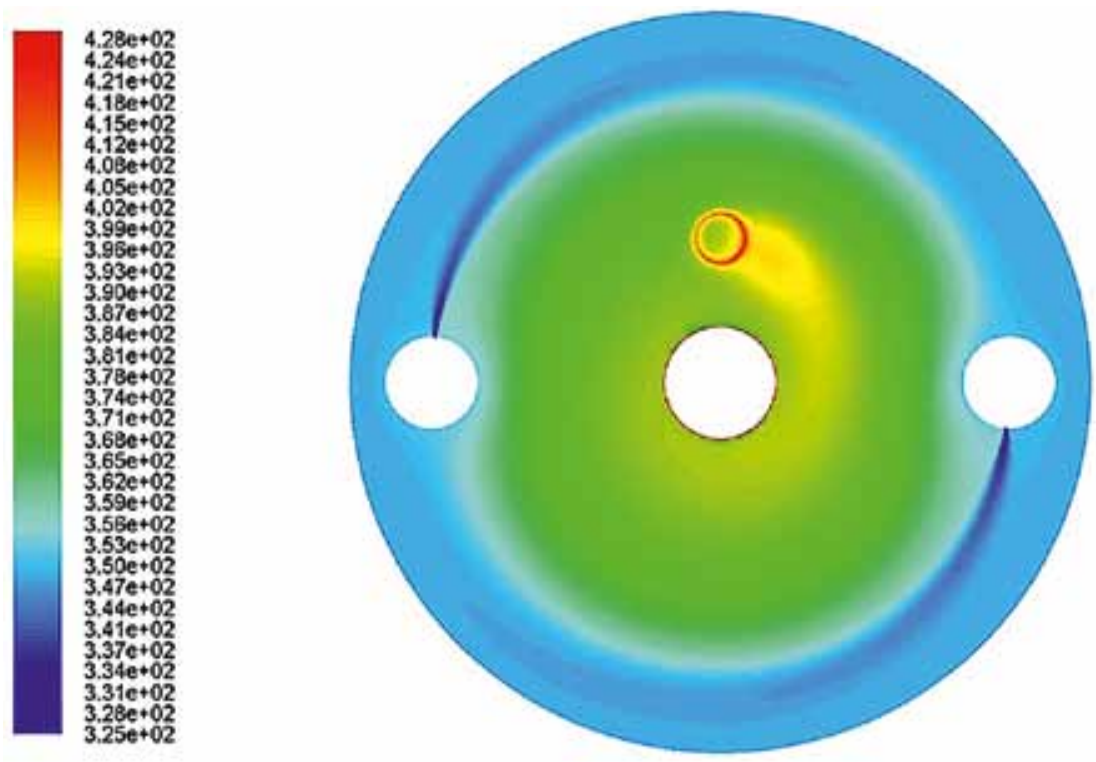

\section{CRAB CAVITY SYSTEM}

A final critical component of the beam delivery system is the crab cavity system, which effectively provides head-on bunch collisions at the interaction point by compensating for the 14-mrad interaction region crossing angle. The radiofrequency (RF) transverse deflecting cavity 'tilts' the bunches at the interaction point. Design and prototyping of the crab cavity has recently demonstrated that the key performance characteristic of the system - the relative phase stability between electron and positron crab-cavities - can be achieved. RF tests of the prototype system have demonstrated an rms phase stability better than 0.1 degrees [3-85], which is already close to the required ILC specification (0.08 degrees). Further improvements have been identified.

\subsubsection{Beam dynamics and simulation}

The luminosity performance of the ILC relies heavily on the ability to preserve the ultra-low emittances produced in the damping rings as the beams are transported and accelerated to the interaction point. The major sources of performance degradation arise from component alignment and instrumentation errors. Quantifying the expected performance can only be made via sophisticated simulations, ideally of the whole accelerator, which include models of the various tuning algorithms to be applied in the real machine. 
The work continues with refinement of the models, and inclusion of new effects. In this way the simulation models become more realistic, and confidence is gained in the projected performance. Since publication of the Reference Design Report, many design modifications have also been proposed and accepted (see chapter 4 ), and the impact of these modifications on the beam dynamics generally needs re-evaluation.

For the ring-to-main-linac system, two major changes in configuration have been considered: a single-stage bunch compressor instead of the two-stage bunch compressor considered for the RDR, and lattice modification in the central area necessitated by the new layout of damping rings. Additional beam dynamics effects extensively studied since the RDR include coupler wakefield and RF kicks in the superconducting cavities [3-86, 87, 88].

During the studies for the RDR, the effort's main focus was on the main linacs and the beam delivery system. The ring-to-main-linac - which includes the long transport line from the damping rings, a 180-degree turnaround, spin rotation sections and bunch compressor - had not been studied in any great detail, but this has since been rectified. The results of emittance preservation studies for the section of the ring-to-main-linac up to but not including the bunch compressor are shown in Figure 3.37. The final average vertical emittance growth is $5.36 \mathrm{~nm}$, or approximately $27 \%$ of the damping ring extracted emittance of $20 \mathrm{~nm}$.

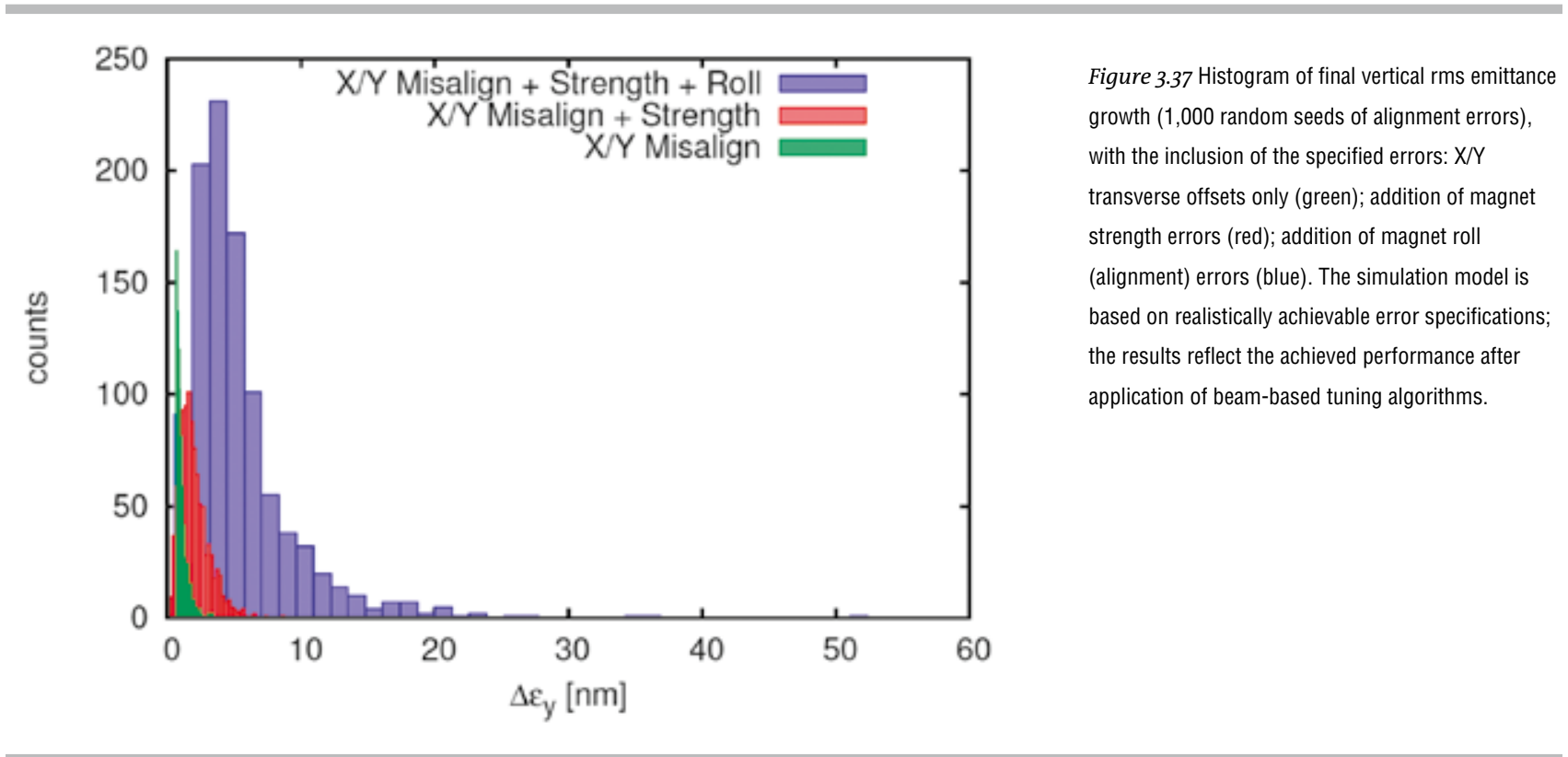

The average vertical emittance growth in the single-stage bunch compressor itself (including all errors) is $2.3 \mathrm{~nm}$, after application of dispersion-free steering, dispersion-generating trajectory bumps and small adjustments to the cryomodule tilt in the RF sections of the compressor. The vertical emittance growth for the entire ring-to-main-linac does not exceed $7.6 \mathrm{~nm}$ on average in the current simulations. 
Table 3.6 Simulated effects of some dynamic (i.e., time-dependent) errors in the main linacs
The effect of possible time-varying stray magnetic fields in the long transport line was found to be quite significant; uncorrected field variations of about 5 nanoteslas rms results in vertical beam jitter of about 50 percent of the beam size. This induces emittance growth of about $1 \mathrm{~nm}$ in the turnaround, though the orbit jitter downstream will be corrected after feed-forward correction [3-89]. Experimental data shows that stray field at frequencies above $1 \mathrm{~Hz}$ can be controlled at this level, but more studies are needed on this issue [3-90].

For the main linacs, the impact of static uncorrelated random errors has been well studied using simulations for the RDR, with the average emittance growth typically measuring around $5 \mathrm{~nm}$ [3-91]. There is possible scope to reduce this further with the application of additional tuning strategies. More recent studies on long-range alignment- and survey-correlated errors [3-92] have indicated potential problems, but these appear to be strongly dependent on the survey and alignment models used and are currently inconclusive.

Effects of coupler wakefield and RF kicks have also been studied in the main linacs. The effects are much less than in the bunch compressors because of the shorter bunch length and are not considered serious.

Effects of dynamic (i.e., time-dependent) errors have been studied as well. Assumed dynamic errors and expected orbit change and emittance growth due to each of the errors are summarised in Table 3.6.

\begin{tabular}{|c|c|c|c|}
\hline & Assumption & $\begin{array}{l}\text { Beam jitter at } \\
\text { linac exit }\end{array}$ & $\begin{array}{l}\text { Emittance dilution at } \\
\text { linac exit }\end{array}$ \\
\hline Quad offset change (vibration) & $100 \mathrm{~nm}$ & $1.5 \mathrm{~s}$ & $0.2 \mathrm{~nm}$ \\
\hline Magnet strength jitter & $10^{-4}$ & $1 \mathrm{~s}$ & $0.1 \mathrm{~nm}$ \\
\hline Cavity tilt change & $3 \mathrm{mrad}$ & $0.8 \mathrm{~s}$ & $0.5 \mathrm{~nm}$ \\
\hline Cavity-to-cavity strength change & $1 \%$ & $0.8 \mathrm{~s}$ & $0.5 \mathrm{~nm}$ \\
\hline
\end{tabular}

1 Note that the radiofrequency feedback control corrects the sum energy gain of many cavities to a $0.1 \%$ level, but not the individual cavity voltages themselves.
The significance of a change in the accelerating voltage of individual cavities in combination with mechanical tilt of the cavity (static alignment error) particularly within the approximately 1-ms pulse - has recently become a focus of attention. The effect leads to trajectory errors of individual bunches in a bunch train. While these trajectory errors (which are relatively slow over the 1-ms pulse) can easily be corrected with feedback (or feed-forward) at the end of the linac, the variation in trajectories in the main linacs gives rise to emittance growth of individual bunches (in accordance with the numbers in Table 3.6). With the assumption of 300-mrad rms random cavity tilts, the voltages in the individual cavities need to be corrected to the level of a few percent over the pulse, which is quite challenging. Figure 3.38 shows the simulated average emittance growth along a main linac, assuming $5 \% \mathrm{rms}$ accelerating voltage jitter with 300-mrad rms static cavity tilt error, both in the cases without orbit correction and with orbit correction at $50-\mathrm{GeV}$ beam energy [3-93]. 


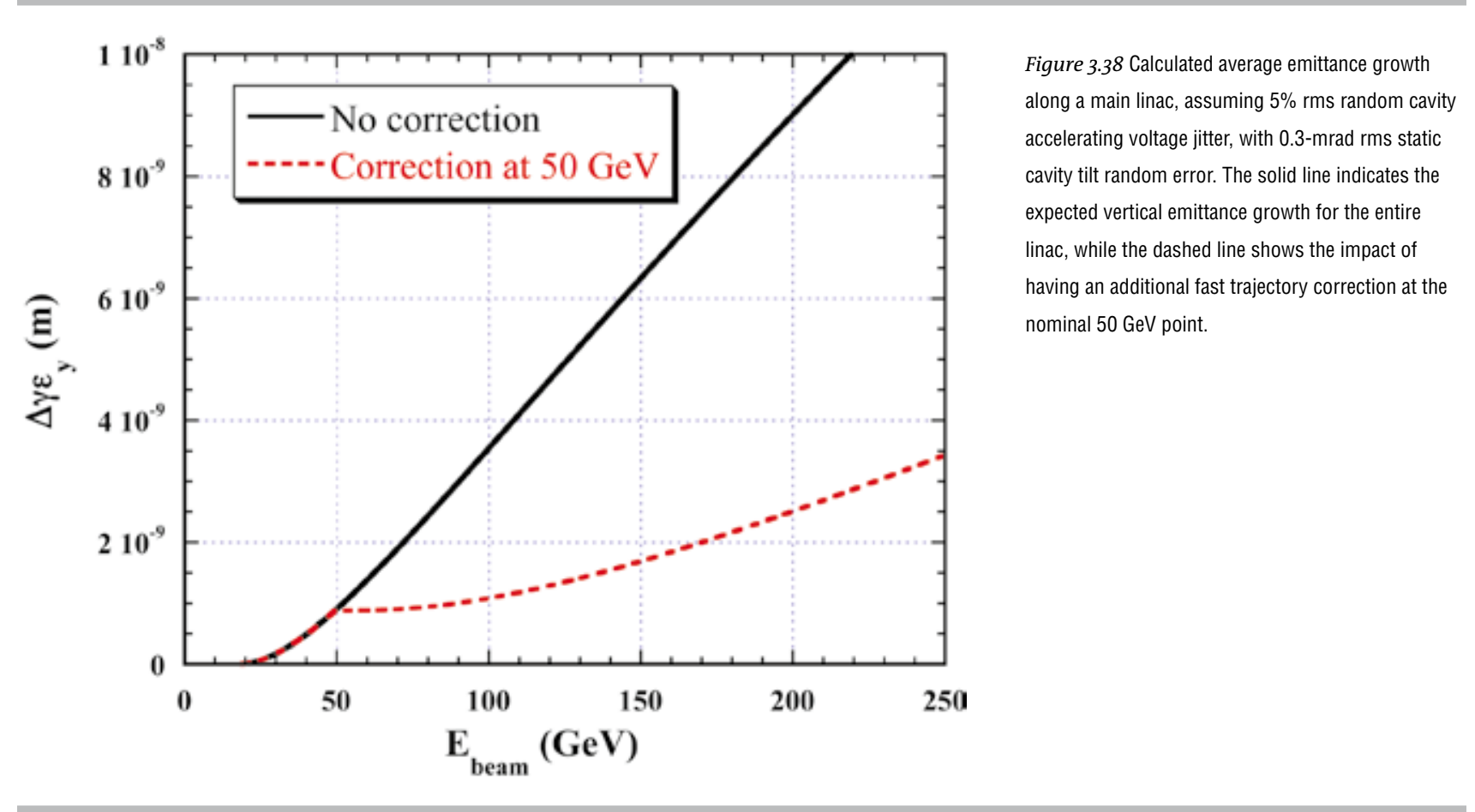

An example of the beam delivery system luminosity tuning simulation for static errors is shown in Figure 3.39. Vertical emittance at the entrance of the beam delivery system was assumed to be $34 \mathrm{~nm}$, which also assumes an emittance growth of $15 \mathrm{~nm}$ for the ring-to-main-linac and main linacs combined. The results show that, for the given assumptions on static errors and input conditions, all random seeds exceed the design luminosity after application of the beam-based tuning algorithms. (Similar simulations have been performed for ATF2, where the results will be experimentally verified.) 


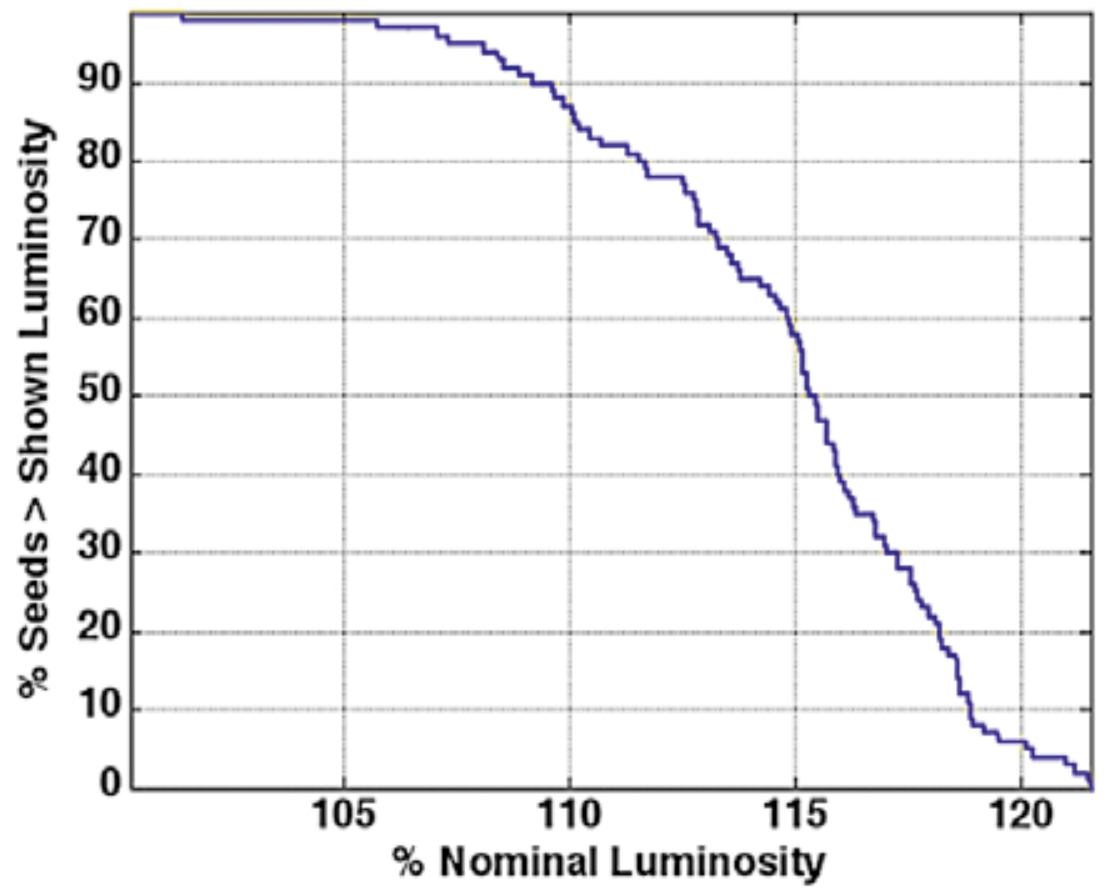

Figure 3.39 Results of simulation of the luminosity tuning in the beam delivery system. The vertical axis indicates the ratio of the random seeds simulated that results in a relative luminosity greater than values in the horizontal axis [3-94].
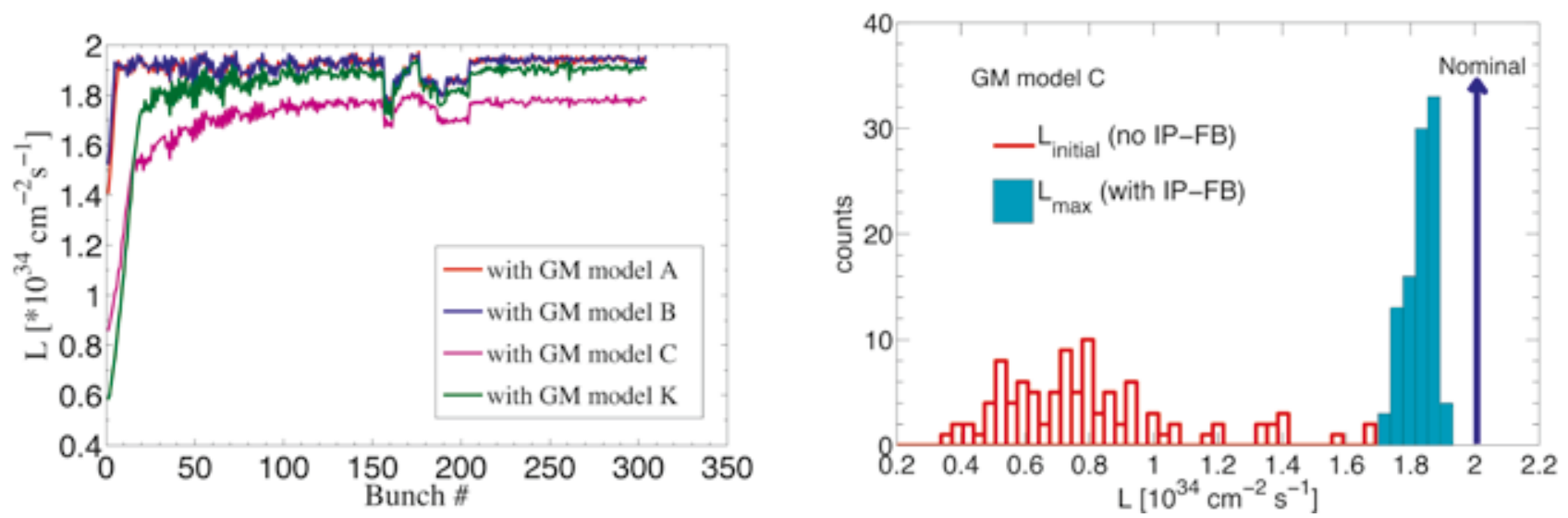

Figure 3.40 Time-dependent luminosity modelling in the beam delivery system. Left: the luminosity is shown as a function of bunch number for the first 300 bunches of a pulse for various ground motion (vibration) models. The luminosity is quickly recovered by the beam-beam fast feedback. Right: a histogram of the results of 100 seeds, assuming model $\mathrm{C}$, as referenced in the left graph, for the ground motion, with and without intra-pulse orbit feedback [3-95].

Effects of dynamic (time-dependent) errors have been also simulated in the beam delivery systems. For example, Figure 3.40 shows the luminosity performances for specific models of ground motion and vibration. The primary effect is a beam-beam offset at the interaction point, which is quickly compensated for by the intratrain fast feedback 
A key parameter is the maximum allowed vibration of the final doublet.

This is primarily set by the limitations of the interaction-point fast feedback,

which becomes increasingly ineffective for larger beam-beam offsets.

Figure 3.41 shows the luminosity as function of rms offset of both final-

doublet cryomodules. The allowed rms final doublet offset tolerances has

been conservatively specified as $50 \mathrm{~nm}$.

Figure 3.41 Simulated luminosity as function of rms offset of both final doublet cryomodules. Mean and standard deviation from tracking simulation are shown [3-94].

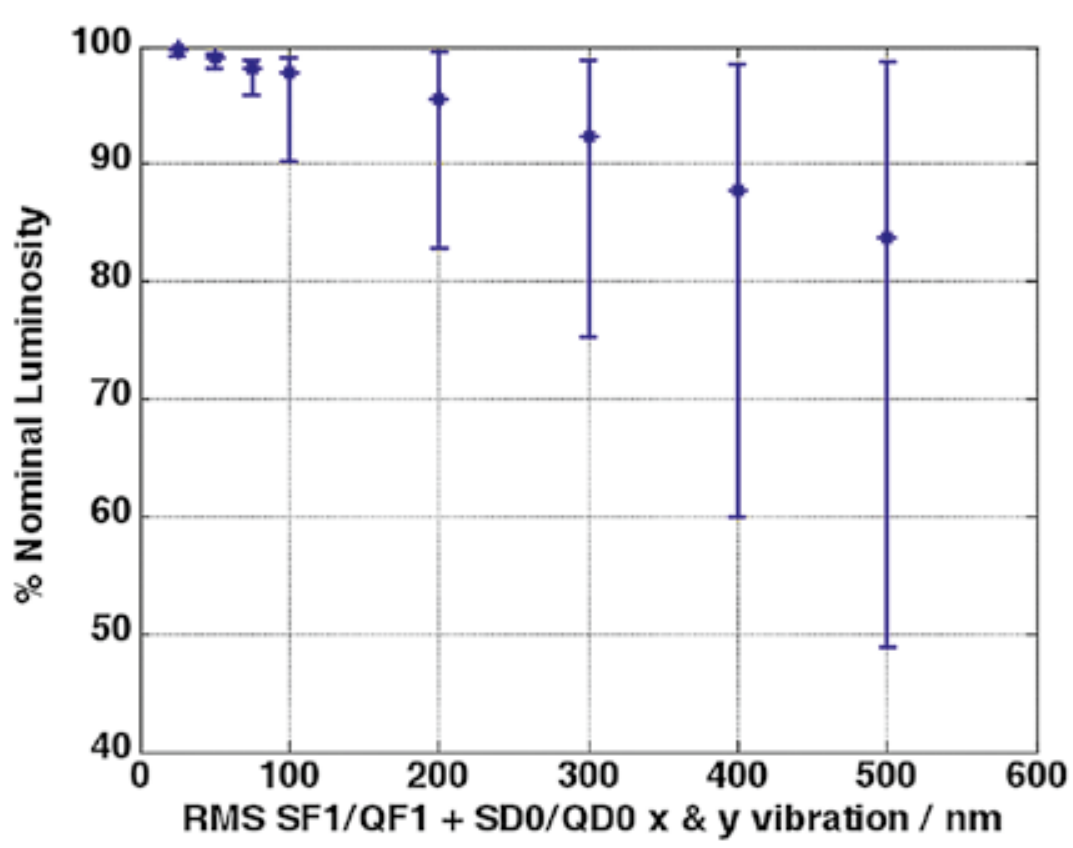

While work continues on a complete integrated simulation, including all static and dynamic effects and tuning and feedback, the current results suggest that the goal luminosity is likely to be achievable to within 10\%, providing the alignment tolerances and other input assumptions in the simulations can be realistically achieved. 
References

[3-1] M.A. Palmer, et al., "Electron Cloud at Low Emittance in CESRTA," Proceedings of IPAC'10, Kyoto, Japan (2010) and references therein.

[3-2] The CesrTA Collaboration includes senior researchers from the following institutions: ANL, Australian Synchrotron, BNL, California Polytechnic State Univ., Carleton Univ., CERN, the Cockroft Institute, FNAL, INFN-LNF, KEK, LBNL, Purdue University, SLAC and Technion-Haifa.

[3-3] http://www.Ins.cornell.edu/Events/ECLOUD10/ WebHome.html

[3-4] https://wiki.lepp.cornell.edu/

ilc/bin/view/Public/DampingRings/

WebHome\#Working_Group_on_Electron_Cloud

[3-5] M.T.F. Pivi, et al., "Recommendation for the Feasibility of More Compact LC Damping Rings," Proceedings of IPAC'10, Kyoto, Japan (2010). [3-6] http://www.Ins.cornell.edu/Events/ECLOUD10/ ILCDampingRingsSatelliteMeeting.html

[3-7] M.A. Palmer, et al., "The Conversion and Operation of the Cornell Electron Storage Ring as a Test Accelerator (CESRTA) for Damping Rings Research and Development," Proc. of PAC09, Vancouver, Canada (2009), and references therein. [3-8] Y. Li, et al., "CesrTA Vacuum System Modifications," Proceedings of PAC09, Vancouver, British Columbia, Canada (2009).

[3-9] D. Rice, "CESR-c: A Wiggler-dominated Collider," Proceedings of PAC07, Albuquerque, New Mexico, USA (2007).

[3-10] M.A. Palmer, et al.., "CESR Beam Position Monitor System Upgrade for CesrTA and CHESS Operations," Proceedings of IPAC10, Kyoto, Japan (2010). [3-11] J. Alexander, et al., "CesrTA X-Ray Beam Size Monitor Design," Proceedings of PAC09, Vancouver, British Columbia, Canada (2009). [3-12] M.A. Palmer, et al., "Design, Implementation and First Results of Retarding Field Analyzers Developed for the CesrTA Program," Proceedings of PAC09, Vancouver, British Columbia, Canada (2009). [3-13] Y. Li, et al., "Design and Implementation of CesrTA Superconducting Wiggler Beampipes with Thin Retarding Field Analyzers," Proceedings of PAC09, Vancouver, British Columbia, Canada (2009).

[3-14] S. De Santis, et al., "The TE Wave Transmission Method for Electron Cloud Measurements at CesrTA," Proceedings of PAC09, Vancouver, British Columbia, Canada (2009).

[3-15] M.G. Billing, et al., "Techniques for Observation of BeamDynamics in the Presence of an Electron Cloud," Proceedings of IPAC'10, Kyoto, Japan (2010).
[3-16] D. Rubin, et al., "CesrTA Layout and Optics,"

Proceedings of PAC09, Vancouver, British Columbia, Canada (2009).

[3-17] J.R. Calvey, et al., "CesrTA Retarding

Field Analyzer Measurements in Drifts, Dipoles, Quadrupoles and Wigglers," Proceedings of IPAC'10, Kyoto, Japan (2010).

[3-18] J.R. Calvey, et al., "Electron Cloud Mitigation Investigations at CesrTA," to be published in the proceedings of the ECLOUD'10 workshop, Ithaca, New York, USA (2010).

[3-19] J.R. Calvey, et al., "CesrTA Retarding Field Analyzer Modeling Results," Proceedings of IPAC'10, Kyoto, Japan (2010).

[3-20] J.R. Calvey, et al., "Methods for Quantitative Interpretation of Retarding Field Analyzer Data," to appear in the Proceedings of the ECLOUD10 Workshop, Ithaca, New York, USA (2010).

[3-21] S. De Santis et al., "Characterization of Electron Clouds in the Cornell Electron Storage Ring Test Accelerator using TE-Wave Transmission," Phys. Rev. ST Accel. Beams 13:071002 (2010).

[3-22] G. Penn, J-L. Vay, "Theoretical Studies of TE-Wave Propagation as a Diagnostic for Electron Cloud," Proceedings of IPAC'10, Kyoto, Japan (2010). [3-23] K. Sonnad, et al., "Simulations Using VORPAL on the Effect of Imperfections and Nonuniformities in TE Wave Propagation Through Electron Clouds," to appear in the Proceedings of the ECLOUD10 Workshop, Ithaca, NY, USA (2010).

[3-24] S. Veitzer, et al., "Modeling Electron Cloud Buildup and Microwave Diagnostics Using Vorpal," to appear in the Proceedings of the ECLOUD10 Workshop, Ithaca, NY, USA (2010).

[3-25] J. Crittenden, et al., "Electron Cloud Modeling Results for Time-resolved Shielded Pickup Measurements at CesrTA," to appear in the Proceedings of the ECLOUD10 Workshop, Ithaca, NY, USA (2010).

[3-26] Y. Suetsugu, et al., "Demonstration of Electron Clearing Effect by Means of Clearing Electrodes and Groove Structures in High-Intensity Positron Ring", proc. of PAC09, Vancouver, Canada (2009). [3-27] C. Yin Vallgren, et al., "Amorphus Carbon Coatings for Mitigation of Electron Cloud in the Cern SPS," Proceedings of IPAC'10, Kyoto, Japan (2010). [3-28] L. Wang and M.T.F. Pivi, "Electron Trapping in Wiggler and Quadrupole Magnets of CesrTA," Proceedings of IPAC'10, Kyoto, Japan (2010). [3-29] J.P. Shanks, et al., "CesrTA Low Emittance Tuning," Proceedings of IPAC'10, Kyoto, Japan (2010).
[3-30] D.P. Peterson, et al., "CesrTA X-Ray Beam Size Monitor Operation," Proceedings of IPAC'10, Kyoto, Japan (2010).

[3-31] J. Crittenden, et al., "Studies of the Effects of Electron Cloud Formation on Beam Dynamics at CesrTA," Proceedings of PAC09, Vancouver, British Columbia, CA (2009).

[3-32] J. Crittenden, et al., "Progress in Studies of Electron Cloud - Induced Optics Distortions at CesrTA," Proceedings of IPAC'10, Kyoto, Japan (2010).

[3-33] G. .Dugan, et al., "Synrad3D Photon

Propagation and Scattering Simulation," to appear in the Proceedings of the ECLOUD10 Workshop, Ithaca, NY, USA (2010).

[3-34] G. .Dugan, et al., "CesrTA EC-Induced Beam Dynamics," to appear in the Proceedings of the ECLOUD10 Workshop, Ithaca, NY, USA (2010). [3-35] J.W. Flanagan, et al., "Measurement of Low-Emittance Beam with Coded Aperture X Ray Optics at CesrTA," Proceedings of IPAC'10, Kyoto, Japan (2010).

[3-36] J.W. Flanagan, et al., "xBSM Bunch-by-Bunch Measurements in EC Conditions at CesrTA," to appear in the Proceedings of the ECLOUD10 Workshop, Ithaca, NY, USA (2010).

[3-37] K. Ohmi, et al., "Electron Instability in Low Emittance Rings, CesrTA and SuperKEKB," to appear in the Proceedings of the ECLOUD10 Workshop, Ithaca, NY, USA (2010).

[3-38] M.T.F. Pivi, et al., "ILC Damping Ring Electron Cloud R\&D Effort and Single-Bunch Instability Simulations Using CMAD," to appear in the Proceedings of the ECLOUD10 Workshop, Ithaca, NY, USA (2010).

[3-39] M. Palmer, et al., "CesrTA Preliminary

Recommendations for the ILC Positron Damping

Ring," to appear in the Proceedings of the ECLOUD10 Workshop, Ithaca, NY, USA (2010).

[3-40] M. T.F. Pivi, et al, "Secondary Electron Yield Measurements and Groove Chambers Update Tests in the PEP-II Beam Line," 691, proc. of EPAC08, Genova, Italy, 2008.

[3-41] M. T.F. Pivi, et al., "A New Chicane Experiment in PEP-II to Test Mitigations of the Electron Cloud Effect for Linear Colliders," 688, proc. of EPAC08, Genova, Italy, 2008.

[3-42] Y. Suetsugu, et al., Nucl.Instrum. Methods A598 (2008) 372. 
[3-43] Y. Suetsugu, et al., "Experimental Studies on Grooved Surfaces to Suppress Secondary Electron Emission," Proceedings of IPAC'10, Kyoto, Japan, 2010. [3-44] Y. Suetsugu, et al, "Beam Tests of a Clearing Electrode for Electron Cloud Mitigation at KEKB Positron Ring," Proceedings of IPAC'10, Kyoto, Japan, 2010. [3-45] D. Alesini, et al., "Design and Test of the Clearing Electrodes for e- cloud Mitigation in the e+ DAFNE Ring," Proceedings of IPAC'10, Kyoto, Japan, 2010. [3-46] M. Pivi, "Recommendation for the ILC DR EC mitigations," slides 20-49, IWLC2010 Workshop, CERN, October 2010, http://ilcagenda.linearcollider. org/materialDisplay. py?contribld $=544 \&$ sessionld $=83 \&$ materialld $=$ slides \& confld $=4507$

[3-47] P. Bambade et al., Phys. Rev. ST Accel. Beam 13, 042801 (2010), and references therein. [3-48] V. Balakin and A. Mikhailichenko, Preprint INP-79-85, 1979. See also K. Flöttmann, DESY-93$161,1993$.

[3-49] Y. Ivanyushenkov et al, PAC 2007, Albuquerque, p2865.

[3-50] A. Mikhailichenko, PAC 2007, Albuquerque, p1974.

[3-51] E. Baynham et al, PAC 2009, Vancouver. [3-52] J. A. Clarke, http://ilcagenda.linearcollider.org/ getFile. py/access? contribld=462\&sessionld=77\& resld= 0\&materialld=slides\&confld=4507, IWLC 2010, Geneva. [3-53] G. Ellwood, http://indico.cern.ch/getFile.py/ access? contribld=6\&res $\mid d=0 \&$ materialld $=$ slides \& confld=109166, EUCARD-WP7-HFM collaboration meeting, Nov 2010, CERN.

[3-54] S.P. Antipov et al., PAC 2007 Albuquerque, p2909. [3-55] I.R. Bailey et al., EPAC 2008, Genoa, p715. [3-56] I.R. Bailey et al., IPAC 2010, Kyoto, p4125. [3-57] I.R. Bailey, http://ilcagenda.linearcollider.org/ getFile.py/access? contribld=485\&sessionld $=83 \&$ resld =0\&materialld=slides\&confld=4507, IWLC 2010, Geneva [3-58] J. Gronberg et al, IPAC 2010, Kyoto, p4137. [3-59] Wanming Liu and Wei Gai, IPAC 2010, Kyoto, p4134.

[3-6o] T. Omori, http://ilcagenda.linearcollider.org/ getFile. py/access? contribld=475\&sessionld=77\& resld= 1\&materialld=slides\&confld=4507, IWLC 2010, Geneva. [3-61] T. Takahashi,

http://ilcagenda.linearcollider.org/materialDisplay.py?c ontribld $=471 \&$ sessionld $=77 \&$ materialld $=$ slides \&co nfld=4507, IWLC2010, Geneva.
[3-62] V. Yakimenko, http://ilcagenda.linearcollider. org/getFile.py/access? contribld $=487 \&$ sessionld $=83 \& \mathrm{r}$ esld $=1 \&$ materialld $=$ slides \& confld $=4507$, and T. Omori, http://ilcagenda.linearcollider.org/getFile.py/access?co ntribld $=486 \&$ session $\mid d=83 \&$ res $\mid d=0 \&$ materiall $d=$ slide s\&confld=4507, IWLC2010, Geneva.

[3-63] J. Urakawa, "Results From DR and Instrumentation Test Facilities," Proceedings of PAC2005, Knoxville, Tennessee, 2005

[3-64] J. P. Shanks, D. Rubin, D. Sagan, "CesrTA Low Emittance Tuning," Proceedings of IPAC'10, Kyoto, Japan, 2010.

[3-65] D.P. Peterson et al., "CesrTA x-Ray Beam Size Monitor Operation," Proceedings of IPAC'10, Kyoto, Japan, 2010.

[3-66] J.W. Flanagan et al., "Measurement of Low-Emittance Beam with Coded Aperture X Ray Optics at CesrTA," Proceedings of IPAC'10, Kyoto, Japan, 2010.

[3-67] R. Bartolini, "Performance and Trends of Storage Ring Light Sources," Proceedings of EPAC08, Genoa, Italy, 2008

[3-68] R. Bartolini, "Light Sources Trends and Common Design Issues with Low Emittance Rings," LER10, Low Emittance Rings Workshop, CERN 12-15 Jan. 2010. http://indico.cern.ch/materialDisplay.py?c ontribld=92\&session $I d=21 \&$ materiall $d=$ paper $\&$ co nfld $=74380$

[3-69] M. Böge et al., "The Swiss Light Source a "Test-bed" for Damping Ring Optimization," Proceedings of IPAC10, Kyoto, Japan, 2010 [3-70] R. Dowd, et al., "Emittance Coupling Control at the Australian Synchrotron," Proceedings of PAC09, Vancouver, BC, Canada, 2009.

[3-71] G. Rehm, "Recent Development of Diagnostics on 3rd Generation Light Sources," Proceedings of EPAC08, Genoa, Italy, 2008

[3-72] C. Thomas et al., PRSTAB, 13, 022805, 2010

[3-73] A. Andersson, et al, Nucl. Instrum. Methods Phys. Res., Sect. A 591, 437, 2008.

[3-74] T. Naito et al., "Development of a $3 \mathrm{~ns}$ rise and fall time strip-line kicker for the International Linear Collider," NIM A571(2007) 599-607.

[3-75] T. Naito, et al., "Multi-Bunch Beam Extraction Using Strip-Line Kicker at KEK-ATF," Proceedings of IPAC'10, Kyoto, Japan, 2010.

[3-76] D. Alesini et al., "Design and Tests of New Fast Kickers for the DAFNE Collider and the ILC Damping Rings," Proceedings of EPAC 2006, Edinburgh, Scotland, 2006.
[3-77] F. Marcellini, et al., "Tests and Operational Experience with the DAFNE Stripline Injection Kicker," Proceedings of PAC09, Vancouver, BC, Canada, 2009. [3-78] F. Marcellini, et al., "Coupling Impedance of DAFNE Upgraded Vacuum Chamber," Proceedings of EPAC08, Genoa, Italy, 2008.

[3-79] T. Tang, C. Burkhart, "Hybrid MOSFET/Driver for ultra-fast switching," IEEE Trans. on Dielectrics and Electrical Insulation, Aug. 2009, in press.

[3-80] T. Tang, C. Burkhart, "Development of an Adder-Topology ILC Damping Ring Kicker Modulator," Proceedings of PAC09, Vancouver, BC, Canada [3-81] F. Arntz et al., "A Kicker Driver Exploiting Drift Step Recovery Diodes for the International Linear Collider," Proceedings of EPAC08, Genoa, Italy, 2008 [3-82] C. Burkhart, et al., "Ultra-Fast Damping Ring Kicker Modulator Development at SLAC," IWLC2010 Workshop, CERN, October 2010.

[3-83] ILC-NOTE-2009-050

[3-84] BARC references

[3-85] Crab cavity Cockcroft Institute reference [3-86] N. Solyak et al., "Final Results on RF- and Wake- Kicks Caused by the Couplers in the ILC Cavity," ICAP 2009, San-Francisco [3-87] A.Latina et al., "Emittance Dilution Caused by the Couplers in the Main Linac and in the $B C$ of the ILC," PAC 09 [3-88] Dirk Kruecker, ILC-LET Workshop, SLAC, Dec.2007

[3-89] A.Latina, ILC-CLIC Workshop, Geneva, Oct. 2010 [3-9o] K. Kubo, ILC-Asia Note 2006-05, 2006 [3-91] Dmitri Sergatskov, ILC-CLIC LET Beam Dynamics Workshop, CERN, June 2009 [3-92] John Dale, ILC-CLIC LET Beam Dynamics Workshop, CERN, June 2009 [3-93] K. Kubo, ILC-CLIC Workshop, Geneva, Oct. 2010 [3-94] G.White, ILC-LET Workshop, SLAC, Dec.2007 [3-95] J. Resta-Lopez, ILC-CLIC Workshop, Geneva, Oct. 2010 


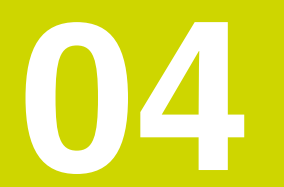

\author{
4.1 EVOLVING DESIGN BEYOND \\ THE REFERENCE DESIGN REPORT \\ 4.2 LAYOUT AND DESIGN \\ 4.3 PARAMETERS
}

\title{
ACCELERATOR DESIGN \& INTEGRATION
}


The design of the ILC continues to evolve from the 'conceptual design' presented in the Reference Design Report (RDR) [4-1] towards a more mature 'technical design'. The ongoing modifications to the design are based on the results of continuing R\&D on technical systems, on engineering design of these systems and their integration with the civil engineering layouts, designs and supporting services.

At the time of the RDR's publication in 2007, it was envisaged that an Engineering Design Report could be completed by late 2010. However, by early 2008 it became clear that the resources required were not available, so the two-stage technical design phase shifted its focus to risk-mitigating $R \& D$ (see chapter 1). Many technical system designs at the time were already evolving with improved performance, and many ideas for perhaps lower-cost schemes were being proposed for study. In order to consolidate and prioritise these developments in an integrated fashion, it was proposed that a modified formal baseline design be developed by the end of Technical Design Phase 1. The new baseline will form the basis of the technical work and updated cost estimate to be published in the Technical Design Report at the end of 2012.

The accelerator design and integration studies in Technical Design Phase 1 have evolved into a total design review of the older RDR parameters, technical and civil system designs and their integrated layouts. The supporting technical R\&D programmes are described in chapters 2 and 3 and development of the civil system designs in section 5. The proposed integrated design and layout is described below in section 4.3.

\subsection{EVOLVING DESIGN BEYOND THE REFERENCE DESIGN REPORT}

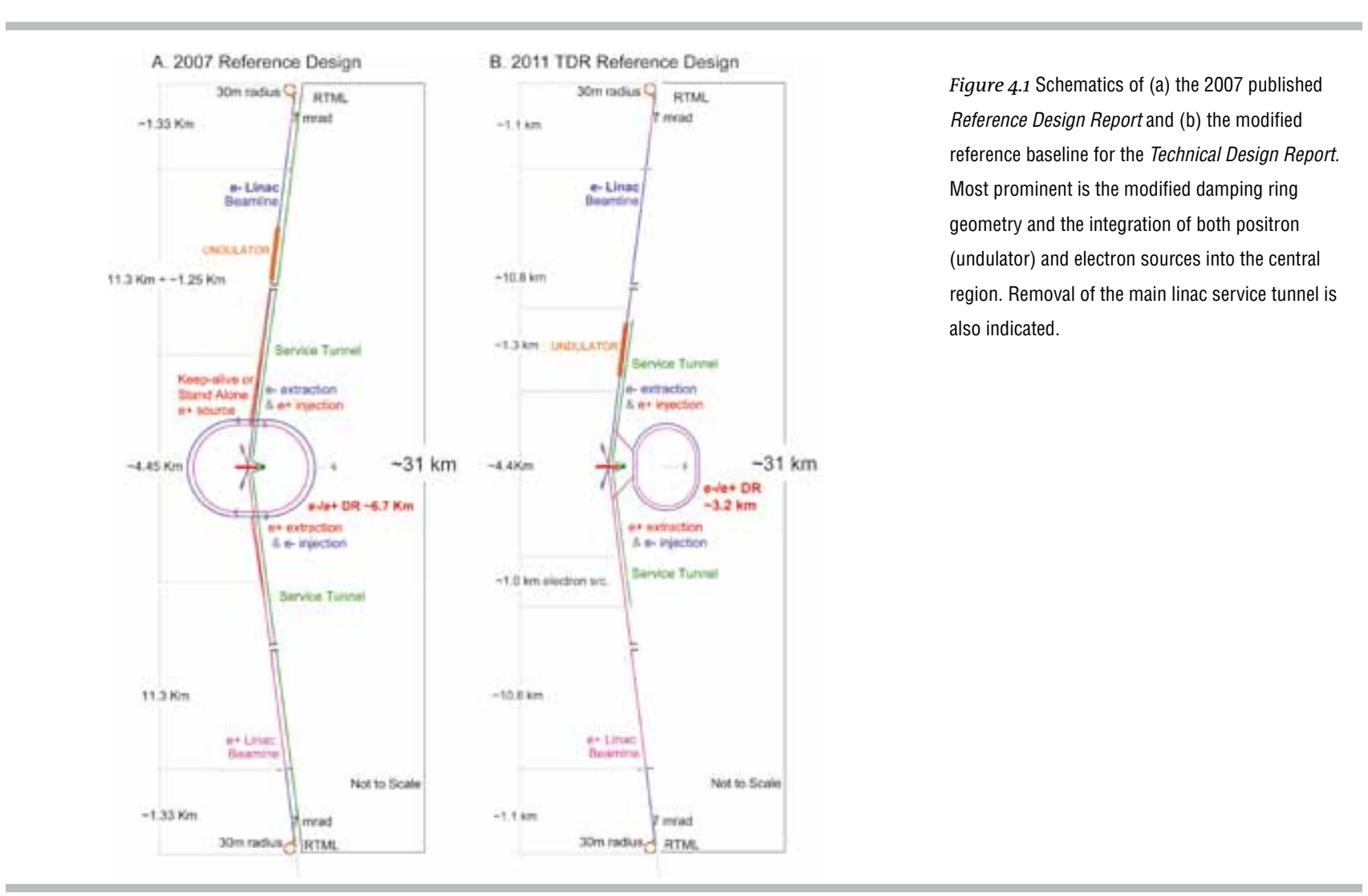




\subsection{LAYOUT AND DESIGN}

\subsubsection{Main linear accelerators}

Schematics of the ILC layout are shown in Figure 4.1 as (a) proposed in the RDR and (b) with the proposed technical and civil engineering changes for 2010. The primary system parameters that have not changed are those of the main electron and positron linacs, which remain the same length, a reflection of the fact that after extensive review, there is no proposed change in the maximum average accelerating gradient of 31.5 megavolts per metre (MV/m). The linear accelerators, or linacs, also remain oriented to provide the 14-milliradian (mrad) crossing angle between the two particle beams required at the interaction point.
Figure 4.2 Single-tunnel solutions for the main linacs: (a) distributed radiofrequency sources, where many small modular 800-kilowatt klystrons, modulators and associated power supplies are all installed in the tunnel; (b) klystron cluster system, where no active RF is installed in the tunnel, and the RF power is brought to the accelerator via long high-power over-moded waveguide system; and (c) the solution adopted for the European X-ray Free Electron Laser (ILC backup solution), where the 10-MW klystrons are installed in the tunnel, but driven by surface-located modulators connected via many high-voltage pulsed cables.
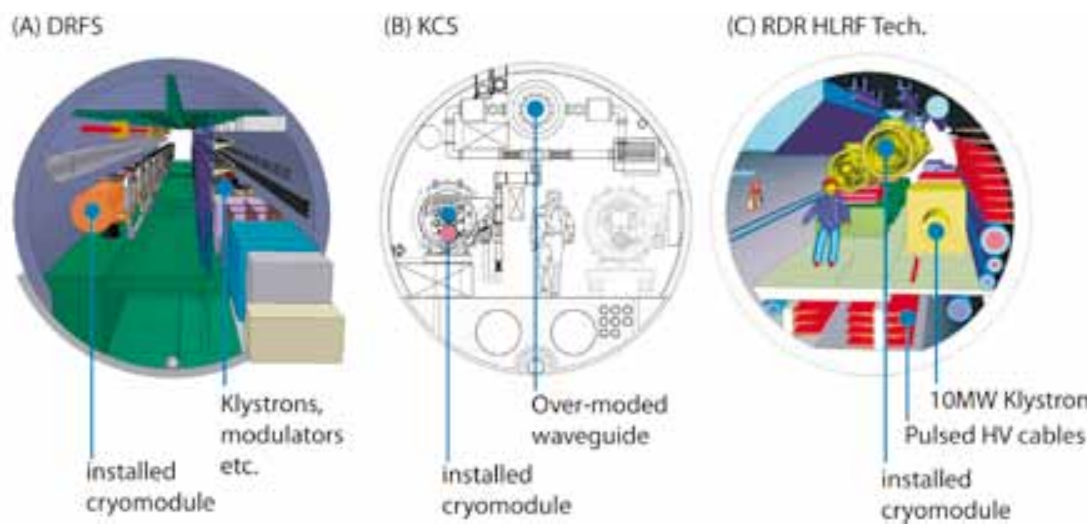

The major change is the removal of the separate so-called service tunnel for the main linacs, in which the radiofrequency (RF) power sources, power supplies and electronics were originally located. This is a significant reduction in underground tunnel volume that benefits both costs and construction schedules. Two novel solutions for the RF power systems have been proposed in support of the single tunnel solution: the distributed radiofrequency sources scheme and the klystron cluster scheme - both of which are described in section 2.5. The two schemes represent quite different solutions for the single-tunnel design and have emerged due to detailed considerations of differing site-specific constraints (see Figure 4.2). Both solutions offer an efficient use of space for the RF power sources and associated power supplies, either in the accelerator tunnel itself (in the case of the distributed radiofrequency sources system), or in surface buildings (klystron cluster scheme). Although these alternate RF power designs appear practical, there is ongoing $\mathrm{R} \& \mathrm{D}$ to demonstrate their performance and cost. As a risk-mitigating measure, a single-tunnel solution based on the RDR/European X-ray Free Electron Laser-type solution is still considered as a backup. 


\subsubsection{Particle sources}

The electron source remains unchanged from that outlined in the RDR as it has the capability of providing any of the electron bunch train patterns or repetition rates being considered in the new baseline options.

The changes in the positron systems are more extensive and are coupled to the layout of the central region, which includes injectors (both electron and positron), damping rings and final focus systems. In the RDR the undulator-based positron production, capture and acceleration systems that accelerate the beam to 400 megaelectronvolts $(\mathrm{MeV})$ were incorporated in an approximately 1.5-kilometre $(\mathrm{km})$ insertion in the electron linac at the nominal 150 gigaelectronvolts $(\mathrm{GeV})$ point (the approximate mid-point). The produced $400-\mathrm{MeV}$ positron beam was then transported $6 \mathrm{~km}$ to the central region (see section 4.2.4) before being accelerated to $5 \mathrm{GeV}$ and injected into the damping ring. This change was considered necessary to maintain an adequate positron yield for operation at centre-of-mass energies of less than $300 \mathrm{GeV}$. An almost duplicate low-charge conventional source (the 'keep-alive source') was originally located in the central region along with the positron and electron $5-\mathrm{GeV}$ booster injectors. Moving the undulator and target systems to the central region, and integrating it with a built-in low-current auxiliary linac source that uses the same target and capture section reduces the total component count and tunnel length.

In addition, going from a linac that has a large acceptance in energy and transverse phase space into such a complex insertion with small acceptance requires beam collimation and machine protection systems ahead of the insertion. These same systems are also required at the ends of the main linacs in the central region to similarly protect the beam delivery systems. Moving the positron system to the central region allows both protection systems on the electron side to be combined into one single system.

Moving the whole positron production to the end of the linac benefits the electron linac operation and civil designs, but the benefits come with trade-offs that require alternative methods of boosting positron yield for lower-energy running. The proposed solution is to run the electron injector and electron linac at $10 \mathrm{~Hz}$ with alternating pulses at $5 \mathrm{~Hz}$, producing either positrons that are sent into the damping ring or electron beams that are sent to the interaction point for collisions. This can be done at low energies with almost no modifications to the electron systems and with electrical power consumption equal to or less than that required at full energy. The original damping time in the damping rings has to be reduced by a factor of two, which can be achieved with the introduction of more wiggler magnets and $\mathrm{RF}$ cavities (and power); the new flexible damping ring design (section 4.2.3) can accommodate these modifications with no changes in the layout of the other systems in the central region. 


\subsubsection{Damping rings}

In the RDR the 6.4-km circumference damping rings had six-fold symmetry. There were difficulties in stacking the two (electron and positron) rings in one tunnel because of physical interferences of components such as wiggler magnets and RF systems. The design rather quickly evolved into a racetrack-shaped design with two 1-km straight sections that contained RF stations, long wiggler magnets and injection and extraction systems. This design allowed many different options for the damping ring layouts and their integration with the other central region systems. For example, in the RDR, to maintain a compact footprint of the central region, the damping rings, including their $5-\mathrm{GeV}$ injector boosters, were in a plane separated vertically by $10 \mathrm{~m}$ from the plane of the linac and final focus. With the racetrack design, everything can remain in one plane, with the injection/ extraction straight section close to and parallel to the final focus beamline, simplifying the civil engineering.

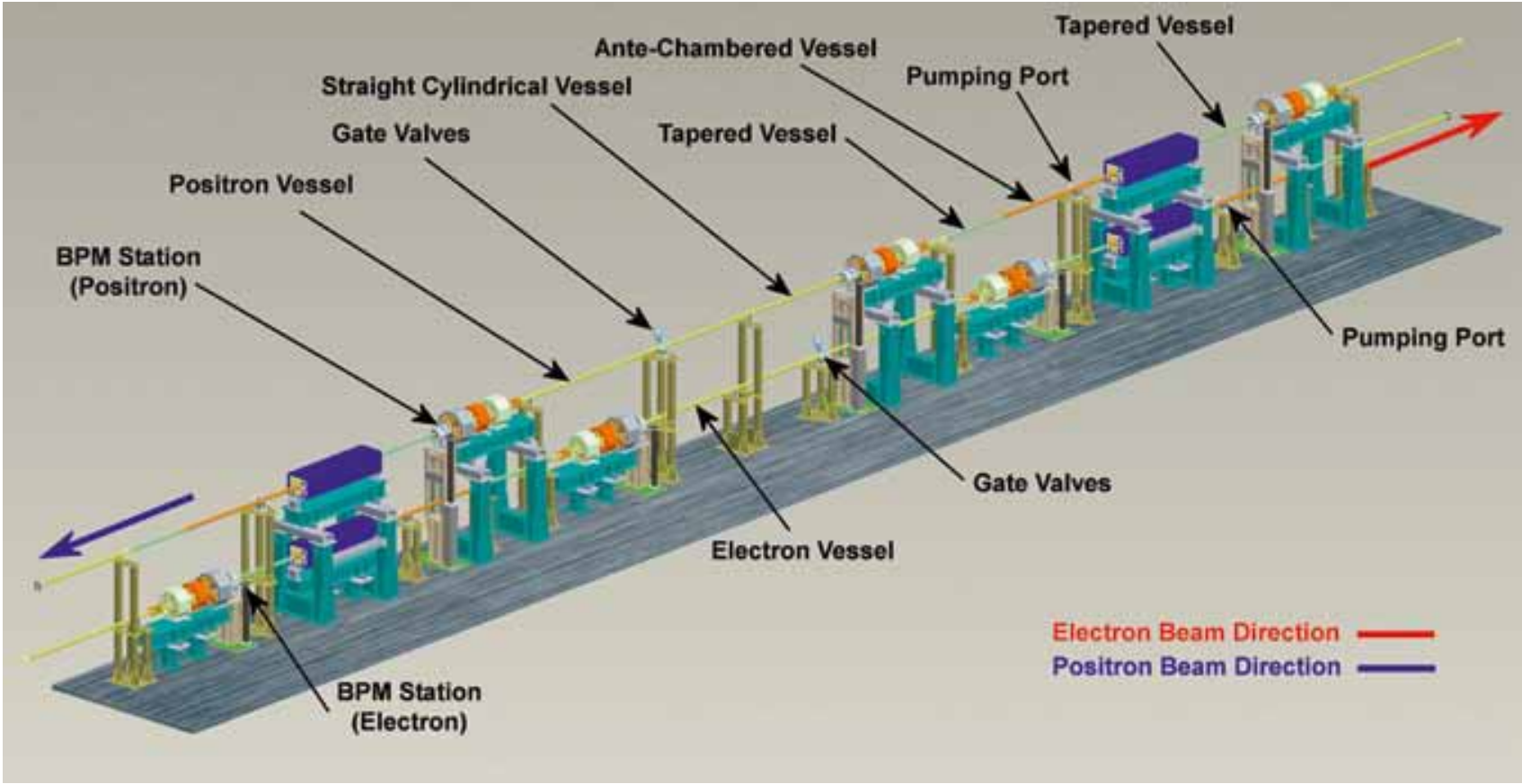

Figure 4.3 Engineering design for the DCO4 damping ring arc cell.

A low and flexible momentum compaction lattice has provided RF system and bunch length optimisation. The 6.4-km DCO4 lattice design is quite mature. It satisfies the main damping ring requirements and has been the basis of a large amount of technical design work (Figure 4.3) [4-2]. At present two lattices for the $3.2-\mathrm{km}$ ring are under study $[4-3,4]$ based on the same racetrack design as the $\mathrm{DCO}_{4}$ and with very similar straight sections, in order to take advantage of the technical design already done. Studies have been carried out to compare the performance of the 3.2- and 6.4-km lattices with respect to the electron cloud instability (see section 3.1). 
One large advantage of the present racetrack approach is that it is relatively straightforward to exchange in the design the two different circumference rings being considered, since both have the same 1-km injection/extraction straight sections. This provides a common interface to the central region integrated layout, which is now independent of the choice of damping ring design. The detailed design of the damping rings will continue to evolve with technology and engineering development as described in sections 3.1 and 3.3.3.

\subsubsection{Central region}

The central region, the central $6 \mathrm{~km}$ of the $30-\mathrm{km}$-long $500-\mathrm{GeV}$ machine, contains all the different ILC systems except the linacs and the ring-tomain-linac transport and bunch compressors. In the RDR layout there were three interconnected tunnels, not counting the damping rings. One tunnel contained the beam delivery systems consisting of complex beamlines and instrumentation, which included several hundred metres of spent-beam transport (after the interaction point) to the main beam dumps. A second tunnel contained the electron and positron (keep-alive) sources, the $5-\mathrm{GeV}$ booster linacs and the injection and return lines to and from the damping rings. The third tunnel was the support tunnel for all of the equipment in the other two tunnels and contained RF equipment, power supplies and instrumentation. This required a complex civil design, which at the time of the RDR was very conceptual.

Today the same equipment, with the undulator source and target replacing the keep-alive positron source, shares a single beam tunnel with the beam delivery system. With respect to the optics and layout of the system, the most significant difference involves modification of the beamline sequence and features for the electron side, primarily to incorporate the positron source (see Figure 4.4). The beam delivery system sacrificial collimators are now protecting the undulator and are placed upstream. The new dogleg needed to separate the gamma beam from the main beam is designed in such a way to minimise beam emittance growth for the beam energy up to $0.5 \mathrm{TeV} /$ beam. The fast abort capability is located in front of the undulator while the tune-up extraction beamline retains only DC extraction.

Figure 4.4 Updated design of the electron beam delivery system, compatible with integration requirements.

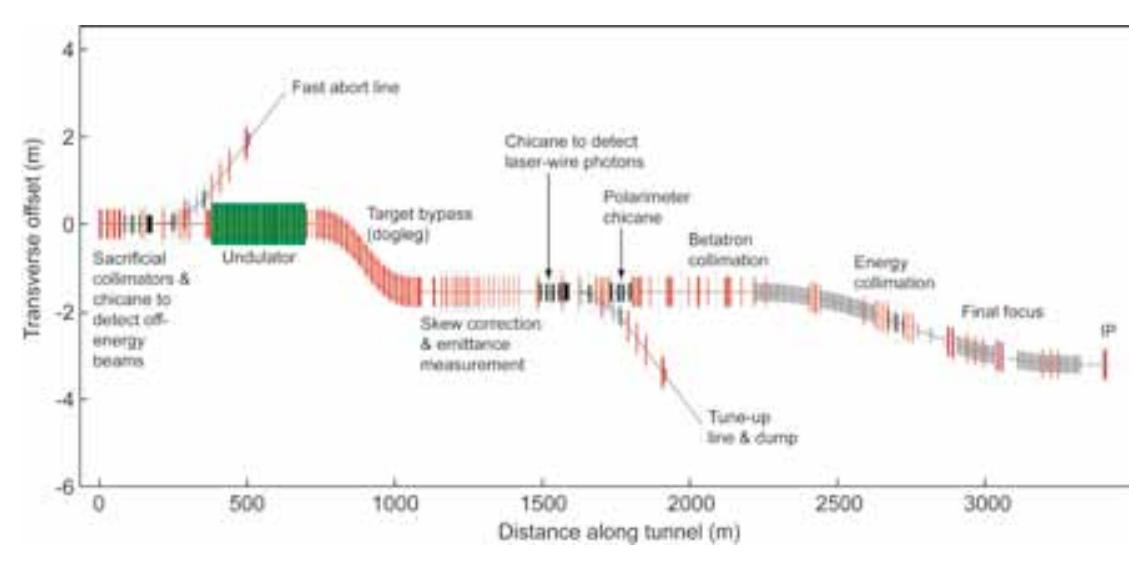




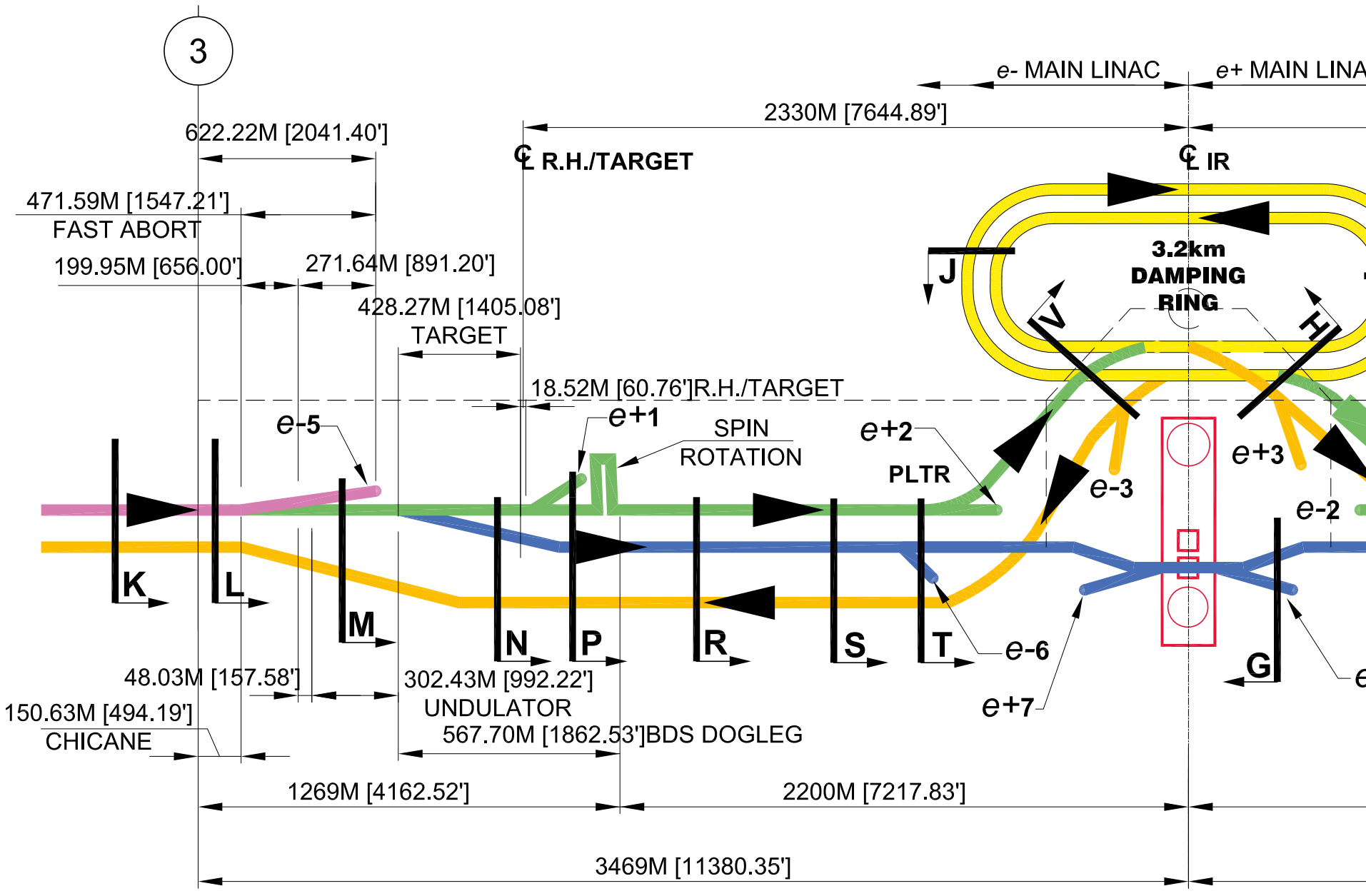

A second equipment support tunnel exists as before and everything is in one plane. There is less underground volume than previously but the central region still has the most complex layout and civil engineering of any section of the ILC (see Figure 4.5).

To achieve practical satisfactory designs, integration of three-dimensional computer-aided design (CAD) modelling of civil engineering and beamline components (geometry) is required. The 3 -D CAD work, along with several iterations of technical and civil designs in special GDE workshops, brings us to the present baseline layout. Designs for the whole $\pm 3 \mathrm{~km}$ of the central region now exist as a baseline for final civil engineering. An impression of a few hundred metres of the central region is shown in Figure 4.6. 
$J^{1}$ 4
2 214M [701.84'] 622.22M [2041.40']

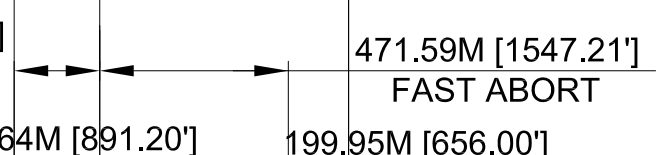

Figure 4.5 Schematic of the various beamlines in the central region, approximately five kilometres from the interaction point (not to scale).

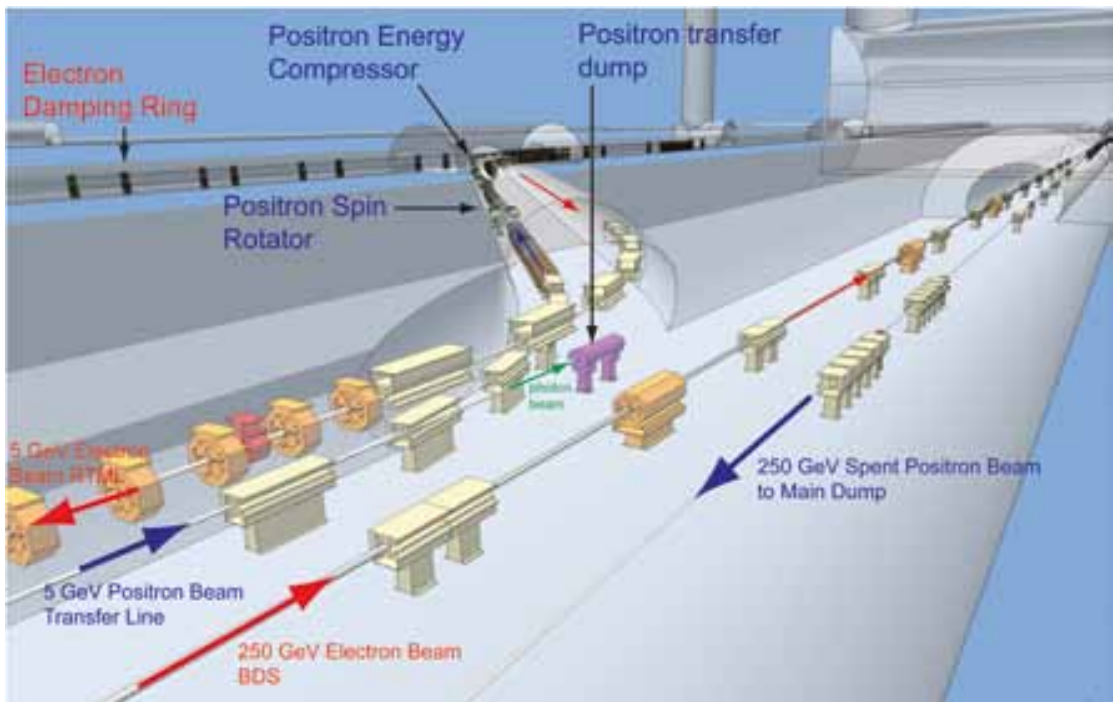

Figure 4. 6 Example of a 3-D layout of part of the central region. 


\subsection{PARAMETERS}

In addition to studying a more cost-effective layout and configuration for the machine as outlined in the previous sections, the accelerator design and integration studies also examined the parameter space of the published reference design with a view to understanding the RDR cost drivers. The performance of the machine design at centre-of-mass energies lower than the nominal $500 \mathrm{GeV}$ has also for the first time been studied in detail. In particular, physics-relevant parameter sets have been developed for centre-of-mass energies of $200,230,250$ and $350 \mathrm{GeV}$, reflecting the physics case centred around a possible light Higgs and the top quark. A modified parameter set that has a reduced beam power has also been developed. This supports a significant cost saving in both RF power generation and the size of the damping rings. Luminosity is restored by more aggressive focusing at the interaction point (beam-beam). Table 4.1 gives the top-level parameters for each of the identified centre-of-mass energies of interest.

\begin{tabular}{|c|c|c|c|c|c|c|c|c|c|}
\hline & Centre-of-mass energy & $\mathrm{E}_{\mathrm{cm}}$ & $\mathrm{GeV}$ & 200 & 230 & 250 & 350 & 500 & upgrade 1,000 \\
\hline & Collision rate & $f_{\text {rep }}$ & $\mathrm{Hz}$ & 5 & 5 & 5 & 5 & 5 & 4 \\
\hline & Electron linac rate & $\mathrm{f}_{\text {linac }}$ & $\mathrm{Hz}$ & 10 & 10 & 10 & 5 & 5 & 4 \\
\hline & Number of bunches & $\mathrm{n}_{\mathrm{b}}$ & & 1,312 & 1,312 & 1,312 & 1,312 & 1,312 & 2,625 \\
\hline & Electron bunch population & $\mathrm{N}$ & $\mathrm{x} 10^{10}$ & 2 & 2 & 2 & 2 & 2 & 2 \\
\hline & Positron bunch population & $\mathrm{N}_{+}$ & $\mathrm{x} 10^{10}$ & 2 & 2 & 2 & 2 & 2 & 2 \\
\hline & Main linac average gradient & $\mathrm{G}_{\mathrm{av}}$ & $\mathrm{MV} / \mathrm{m}$ & 12.6 & 14.5 & 15.8 & 22.1 & 31.5 & $>31.5$ \\
\hline & RMS bunch length & $\sigma_{\mathrm{z}}$ & $\mathrm{Mm}$ & 0.3 & 0.3 & 0.3 & 0.3 & 0.3 & 0.3 \\
\hline & Electron RMS energy spread & $\Delta \mathrm{p} / \mathrm{p}$ & $\%$ & 0.22 & 0.22 & 0.22 & 0.22 & 0.21 & 0.11 \\
\hline & Positron RMS energy spread & $\Delta \mathrm{p} / \mathrm{p}$ & $\%$ & 0.17 & 0.15 & 0.14 & 0.1 & 0.07 & 0.04 \\
\hline & Electron polarisation & $\mathrm{P}$. & $\%$ & 80 & 80 & 80 & 80 & 80 & 80 \\
\hline & Positron polarisation & $\mathrm{P}_{+}$ & $\%$ & 31 & 31 & 31 & 29 & 22 & 22 \\
\hline & IP RMS horizontal beam size & $\sigma_{\mathrm{x}}^{*}$ & $\mathrm{~nm}$ & 904 & 843 & 700 & 662 & 474 & 554 \\
\hline & IP RMS vertical beam size & $\sigma_{\mathrm{y}}^{*}$ & $\mathrm{~nm}$ & 9.3 & 8.6 & 8.3 & 7 & 5.9 & 3.3 \\
\hline & Luminosity & $\mathbf{L}$ & $\times 10^{34} \mathrm{~cm}^{-2} \mathrm{~s}^{-2}$ & 0.47 & 0.54 & 0.71 & 0.86 & 1.49 & 2.7 \\
\hline & Fraction of luminosity in top $1 \%$ & $\mathrm{~L}_{0.01} / \mathrm{L}$ & & $92.20 \%$ & $89.80 \%$ & $84.10 \%$ & $79.30 \%$ & $62.50 \%$ & $63.50 \%$ \\
\hline & Average energy loss & $\delta \mathrm{E}_{\mathrm{BS}}$ & & $0.61 \%$ & $0.78 \%$ & $1.23 \%$ & $1.75 \%$ & $4.30 \%$ & $4.86 \%$ \\
\hline Using & IP RMS vertical beam size & $\sigma_{\mathrm{y}}^{*}$ & $\mathrm{~nm}$ & 6 & 5.6 & 5.3 & 4.5 & 3.8 & 2.7 \\
\hline Travelling & Luminosity & $\mathbf{L}$ & $\times 10^{34} \mathrm{~cm}^{-2} \mathrm{~s}^{-2}$ & 0.64 & 0.73 & 0.97 & 1.17 & 2.05 & 3.39 \\
\hline \multirow[t]{2}{*}{ Focus } & Fraction of luminosity in top $1 \%$ & $\mathrm{~L}_{0.01} / \mathrm{L}$ & & $91.60 \%$ & $89.00 \%$ & $83.00 \%$ & $77.90 \%$ & $60.80 \%$ & $62.30 \%$ \\
\hline & Average energy loss & $\delta \mathrm{E}_{\mathrm{BS}}$ & & $0.61 \%$ & $0.79 \%$ & $1.26 \%$ & $1.78 \%$ & $4.33 \%$ & $4.85 \%$ \\
\hline
\end{tabular}

Table 4.1 Selected top-level parameters assumed for the Technical Design Report. These parameters will be adjusted as the R\&D continues. In particular, the impact of emittance dilution from the main linacs and the beam delivery systems (collimator wakefields) still need to be assessed and included in this table. 
Two sets of luminosity parameters are supplied:

- The first set assumes a straightforward reduction in the vertical beam size at the interaction point, pushing the vertical beta $(\beta)$ function close to the nominally accepted limit of the bunch length. This pushes the vertical disruption parameter to a level close to the instability threshold (approximately 25), but to one that is still considered achievable. This configuration achieves about $75 \%$ of the nominal RDR luminosity $\left(2 \times 10^{34} \mathrm{~cm}^{-2} \mathrm{~s}^{-1}\right)$.

- The second set assumes application of the so-called travelling focus [4-5]. Travelling focus allows the beam to be focused down beyond the traditional limit of the bunch length. Adjustment of the longitudinal position of the focal point (optical waist) of individual longitudinal segments of the bunch effectively compensates the luminosity-diluting effects of the hourglass effect. The technique potentially allows $100 \%$ of the RDR luminosity, but at the cost of a relatively unstable collision due to the intense beam-beam effect, which will result in tighter vibration tolerances and more demanding specifications on beam-based feedbacks. The travelling focus configuration will be studied in more detail during the remainder of the Technical Design Phase.

At centre-of-mass energies of 200,230 and $250 \mathrm{GeV}$, the corresponding beam energies are less than the minimum $150 \mathrm{GeV}$ required to generate the design positron bunch charge (3.2 nanocoulombs). For these energies, the electron linac is run at $10 \mathrm{~Hz}$, with one pulse used to generate a $150-\mathrm{GeV}$ beam to produce positrons, as described in section 4.2.2. (Other alternatives such as a short-period undulator are also under consideration.)

Finally, a tentative parameter set for the $1-\mathrm{TeV}$ upgrade has been added. The current working assumption is that the full RD beam power $(2,625$ bunches) will either be restored in parallel to the energy upgrade or will have already been implemented. Restoration of the full RDR bunch number assumes a doubling of the installed RF power (by klystrons and modulators) and that the damping rings can accommodate the increased bunch number. The major bottleneck for the latter is likely to the electron cloud effects in the positron damping ring. Because of this uncertainty, space is assumed in the current design to facilitate the construction of a second positron ring.

References

[4-1] ILC Reference Design Report, Vol. 3, Accelerator.

[4-2] A. Wolski et al., "ILC Damping Ring

Design Studies at the Cockcroft Institute,"

ILC-NOTE-2010-057.
[4-3] S. Guiducci, M. E. Biagini, "A Low Emittance

Lattice for the ILC $3 \mathrm{~km}$ Damping Ring," Proceedings of IPAC 2010, Kyoto, Japan, 2010.

[4-4] D. Wang, J. Gao, Y. Wang, "A New Design for ILC 3.2 km Damping Ring Based on FODO Cell,"

Proceedings of IPAC 2010, Kyoto, Japan, 2010.
[4-5] V.E. Balakin, Travelling Focus Regime for Linear Collider, VLEPP Proc. 77th ICFA Workshop on Beam Dynamics, May 13-16, Los Angeles, 1991. 


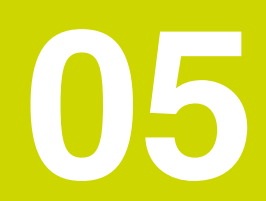

5.1 GLOBAL CONVENTIONAL FACILITIES DESIGN

5.2 LIFE SAFETY AND

INFRASTRUCTURE SUPPORT

5.3 SITE-SPECIFIC DESIGN EFFORTS

\section{CONVENTIONAL FACILITIES AND SITING}


During the Technical Design Phase, the conventional facilities design has continued as a global enterprise coordinating the work in the Americas, Asian and European regions. Over the first two years of this phase, the conventional facilities design has matured from relatively similar generic solutions for sample sites in each of the three regions to more detailed designs adapted to specific site conditions. In all cases, designs are based on requirements that apply to the construction of all modern accelerators: stable geologic conditions for the support of the accelerator complex, suitable radiation shielding, life safety and egress considerations, appropriate infrastructure for the transport of equipment and components and ample utility support. Since a site has not been preselected, the design development in each region has led to differences in the design approach and resulting configuration. These differences will be well documented, cost-estimated and carefully reviewed to understand the distinctions among the regional design solutions.

\subsection{GLOBAL CONVENTIONAL FACILITIES DESIGN}

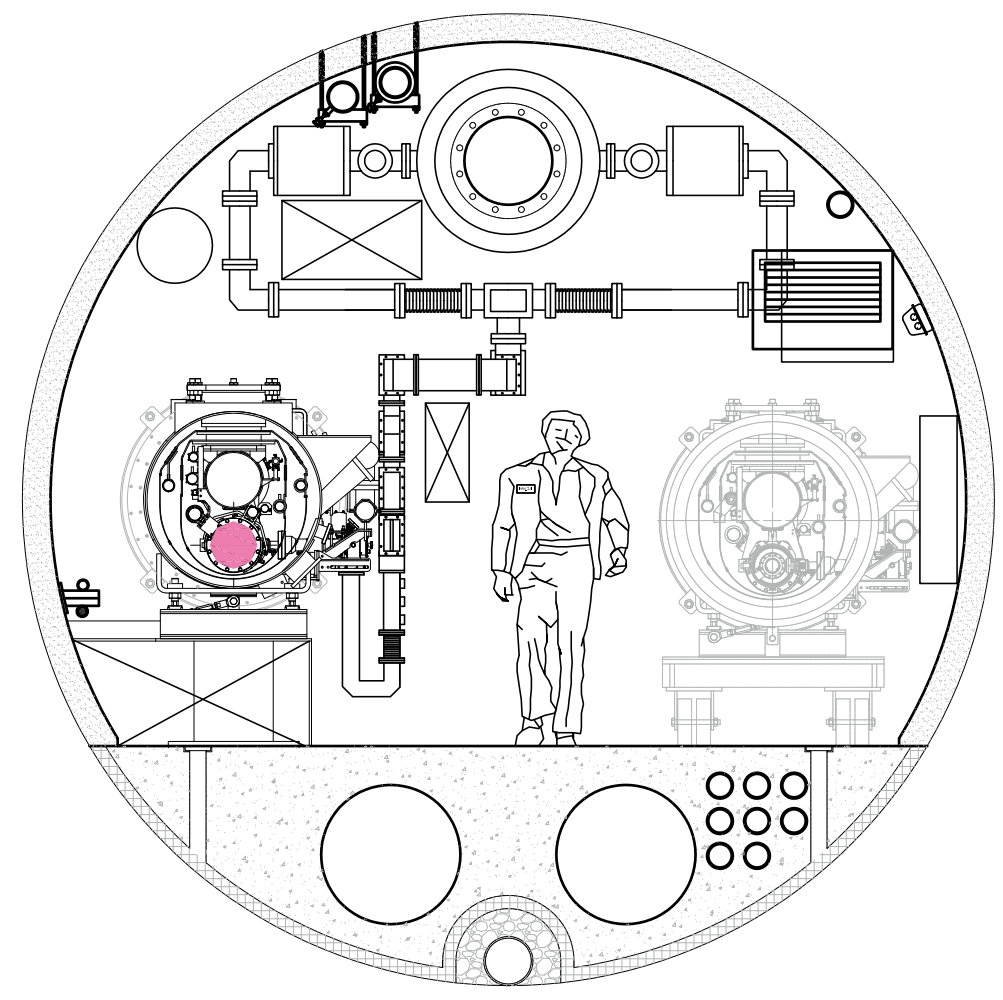

Figure 5.1 Main linac tunnel cross-section for the Americas region. The klystron cluster scheme waveguide is shown.

In Figure 5.2, the overall ILC layout is depicted in schematic form with the path of the electron and positron beams indicated by directional arrows. In all regions, the machine layout and configuration remain consistent. However, regional conditions have prompted differing solutions for tunnel construction as well as the choice of high-level radiofrequency power supply systems. The Americas and European regions have selected a klystron cluster radiofrequency system to facilitate a single-tunnel main linear accelerator (linac) configuration. 


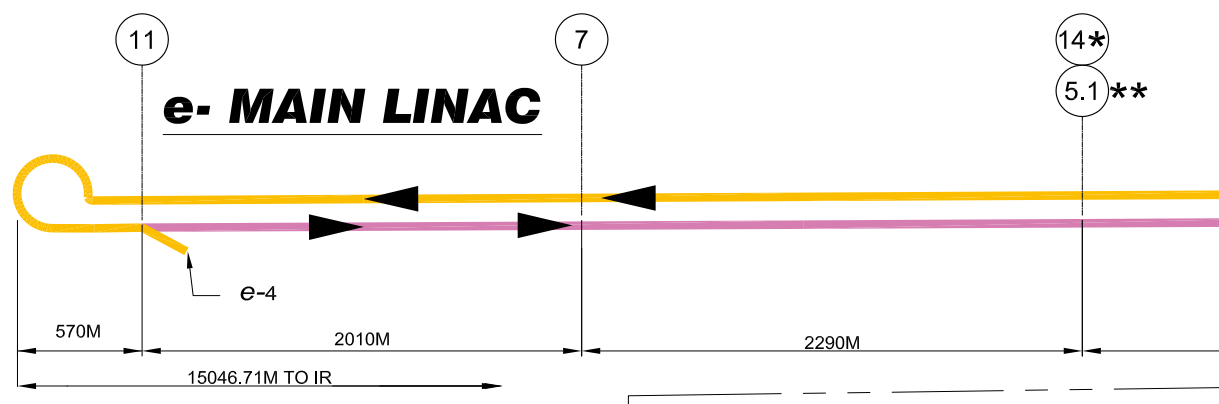

1. e- MAIN LINAC LENGTH DOES NOT ACCOUNT FOR

UNDULATOR ENERGY LOSS.

2. MAIN LINACS LENGTH DOES NOT INCLUDE OVERHEAD.

3 CAVERN DIMENSIONS ARE BASED ON THE AMERICAS GEOLOGIC CONDITIONS.

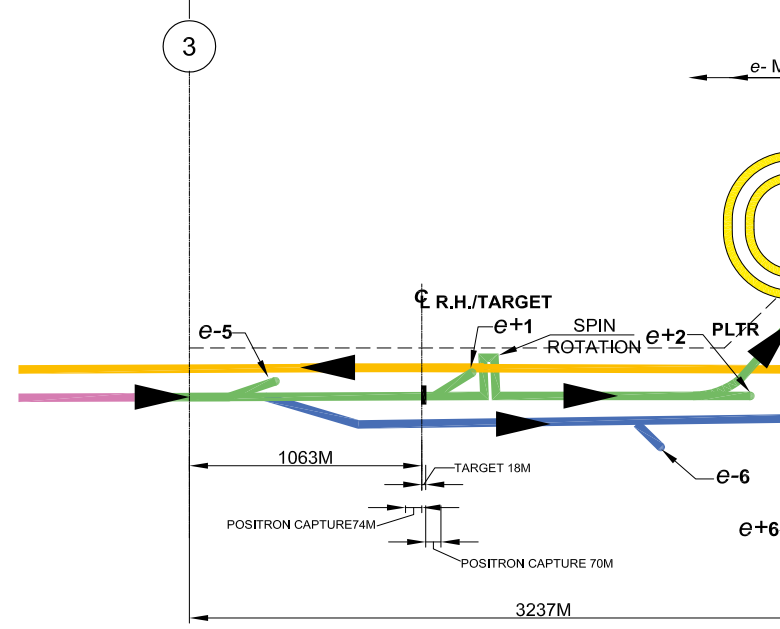

$3237 \mathrm{M}$

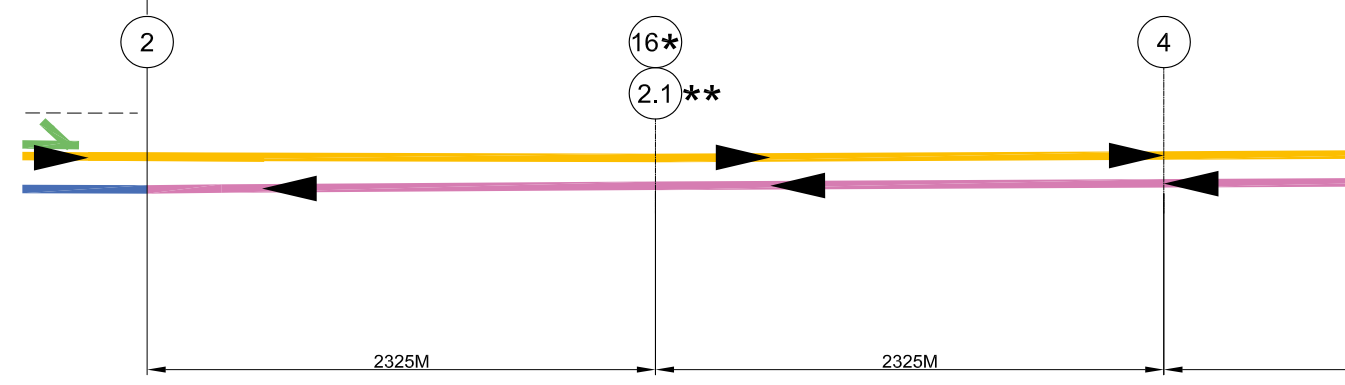

This system allows the klystrons that supply the radiofrequency (RF) power to the accelerating cavities to be grouped in surface buildings located at approximately 2.5-kilometre $(\mathrm{km})$ intervals. Due to regional conditions at sites being considered for the Asian region in Japan, a distributed RF system has been selected for that region. In that system, smaller klystrons are being developed to support only two cavities; these are to be located in the tunnel near the cryomodules. In all regions the primary main linac tunnel diameter is determined by three fundamental dimensions: the space needed for the installed main linac cryomodule, the space needed for transport of replacement equipment and the space required for emergency exit, even in the event that replacement equipment is being transported through the tunnel at the time of an incident. 
Figure 5.3 Main linac tunnel cross-section for the European region showing KCS waveguide.

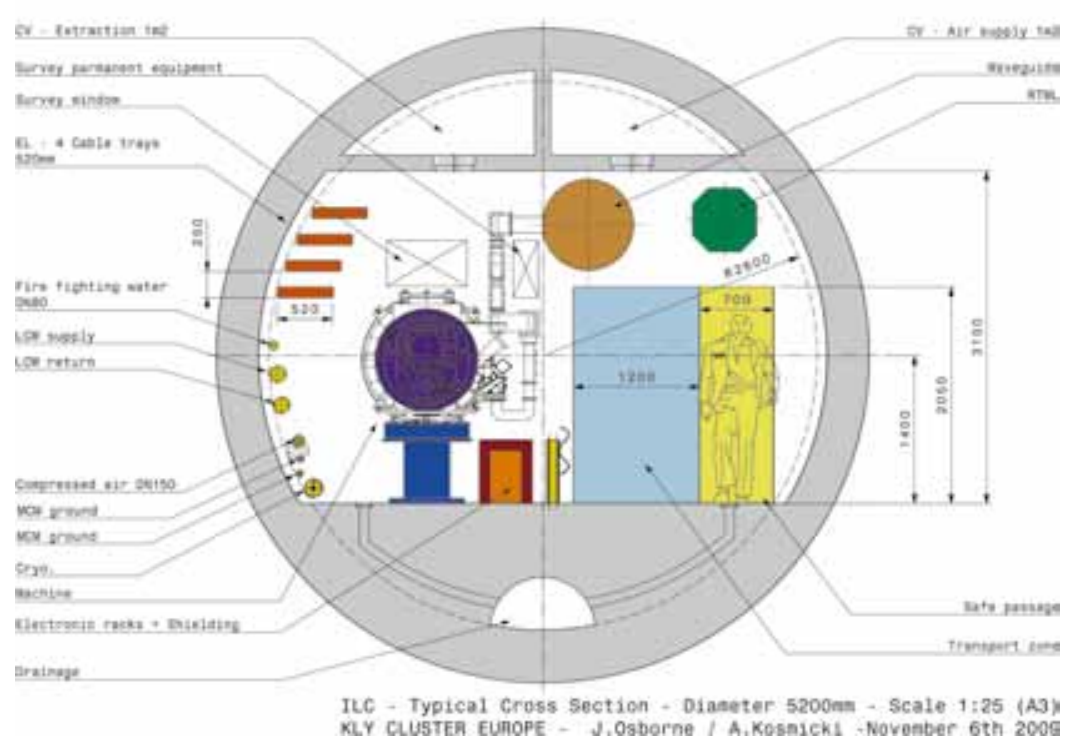

The European main linac tunnel cross-section, Figure 5.3, uses a similar approach to tunnel configuration and also uses the klystron cluster $\mathrm{RF}$ system. The main linac cryomodule is floor-mounted and the large waveguide is also mounted at the tunnel ceiling. The personnel access way and equipment transport space is reversed in position. The main differences between the two cross-sections are the two large supply and return air ducts that are formed at the tunnel crown. The European approach to underground life safety requires a 'compartmentalised' approach in which the underground space is divided into increments of $500 \mathrm{~m}$ and requires the provision for fresh air supply and return for each 'compartment'.
Figure 5.4 Main linac tunnel cross-section for the Asian region showing distributed radiofrequency system installation.

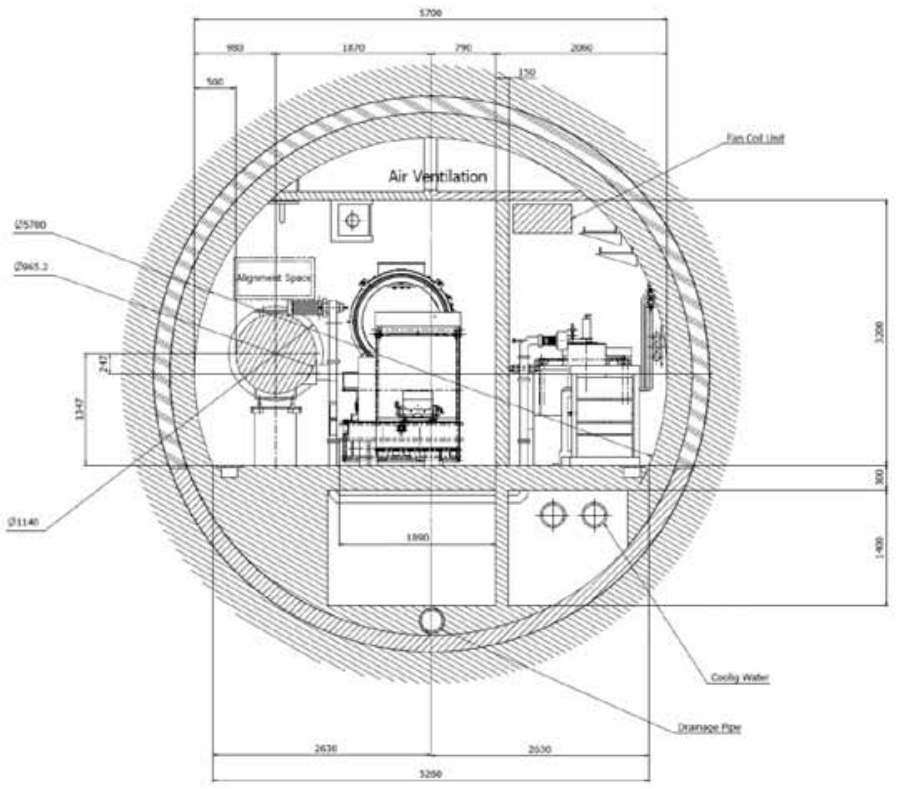


The Asian main linac tunnel cross-section, shown in Figure 5.4, uses a 'compartmentalised' approach to life safety very similar to the European solution. At the crown of the tunnel two large supply and return air ducts are indicated. The Asian main linac tunnel configuration has one major distinction from that of the Americas and European regions. The sites being considered for the ILC in Japan are in mountainous areas and are not suitable for a klystron cluster system approach with large surface buildings. The Asian tunnel cross-section shows the configuration of the distributed RF system. On the left side of the tunnel, the main linac cryomodule is floor-mounted. The centre portion of the tunnel shows an equipment transport vehicle and minimum space for personnel passage. On the right side of the tunnel, the distributed RF system, the small klystrons and related support equipment is indicated. This equipment is separated from the main accelerator space by a wall for radiation shielding. The space below the floor is also utilised for a drainage system to control ground water inflow, process water piping and electrical power cable distribution. The Japanese sites being considered have additional unique features that affect the underground tunnel configuration. These will be discussed later in the site-specific section of the conventional facilities \& siting portion of this report.

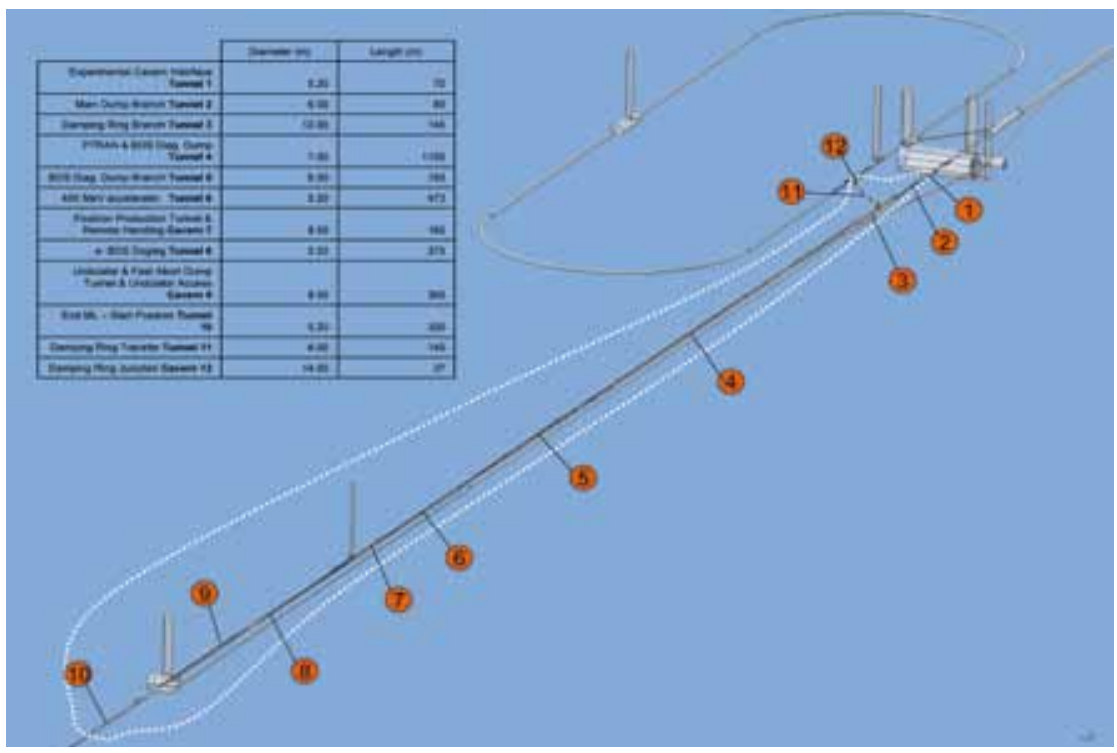

Figure 5.5 An isometric view of the Central Region tunnel schematic.

While the ILC main linacs represent a considerable portion of the underground construction for the project, there are several other requirements for underground space. Both the electron and positron main linacs extend for a length of approximately $10 \mathrm{~km}$ each. However, the 'central region' of the project also occupies a similar overall length of $10 \mathrm{~km}$ and contains the electron and positron sources, the damping rings and the electron and positron beam delivery systems, which lead to the interaction region where two detectors alternate positions in the beamline for data gathering (see Figure 5.2). The electron and positron sources are located in an enlarged enclosure that also accommodates the beam delivery systems. The damping ring is located offset from the interaction region in a racetrack 
shape that has a circumference of $3.2 \mathrm{~km}$. In addition the ring-to-main-linac, which transfers the electron and positron beams from the damping rings to the start of the main linacs, it also occupies space in both the beam delivery system and main linac enclosures. Needless to say, the central region design and construction will require attention to the requirements of each individual system as well as to all aspects of initial installation and ongoing operations and maintenance processes.

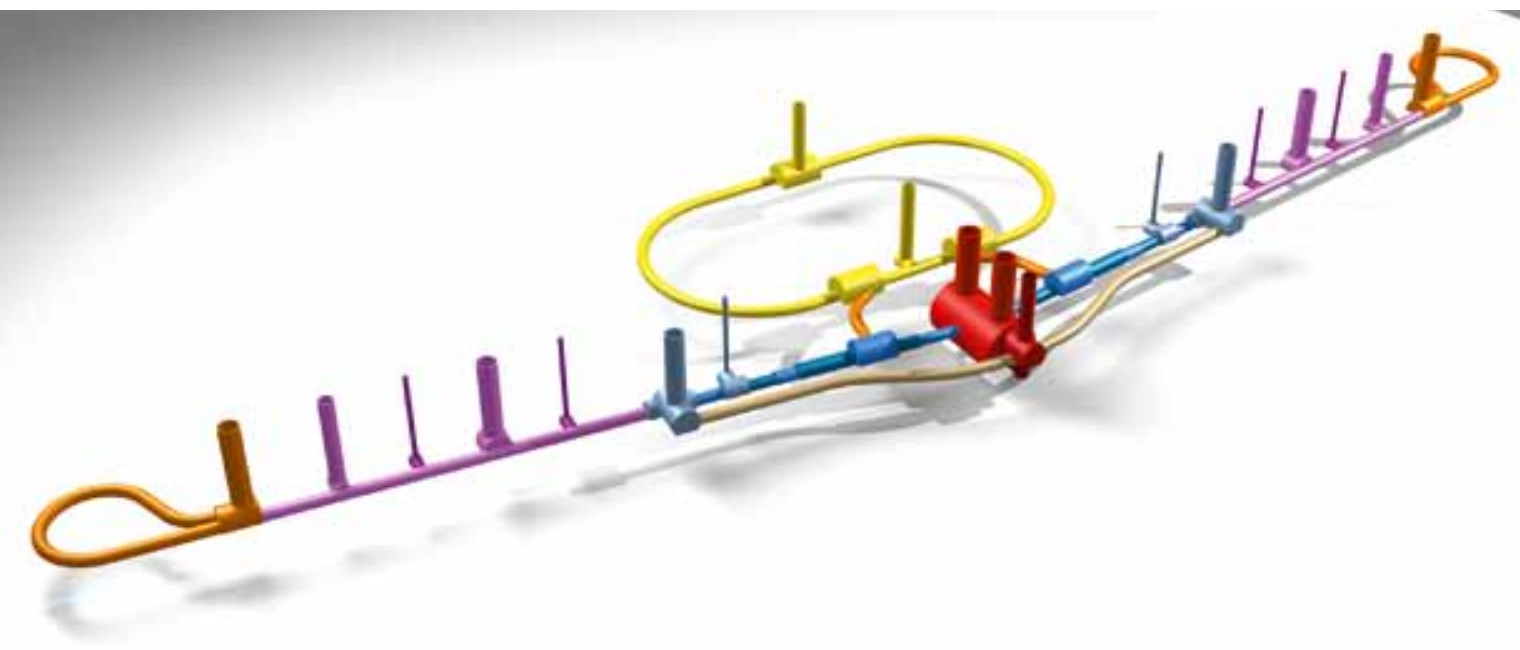

Figure 5.6 An overall view of the linac collider tunnel schematic showing access shafts.

Figure 5.7 An isometric view of the Central Tunnel schematic showing the detector hall.
Figure 5.5 is a 3-D isometric drawing of the electron beam delivery system, damping ring and interaction region. Figure 5.6 is an overview of the entire ILC underground configuration. It is likely that the enclosures for the beam delivery system on each side of the interaction region will have to be enlarged beyond the nominal main linac tunnel diameter to accommodate the accelerator equipment required to occupy this area.

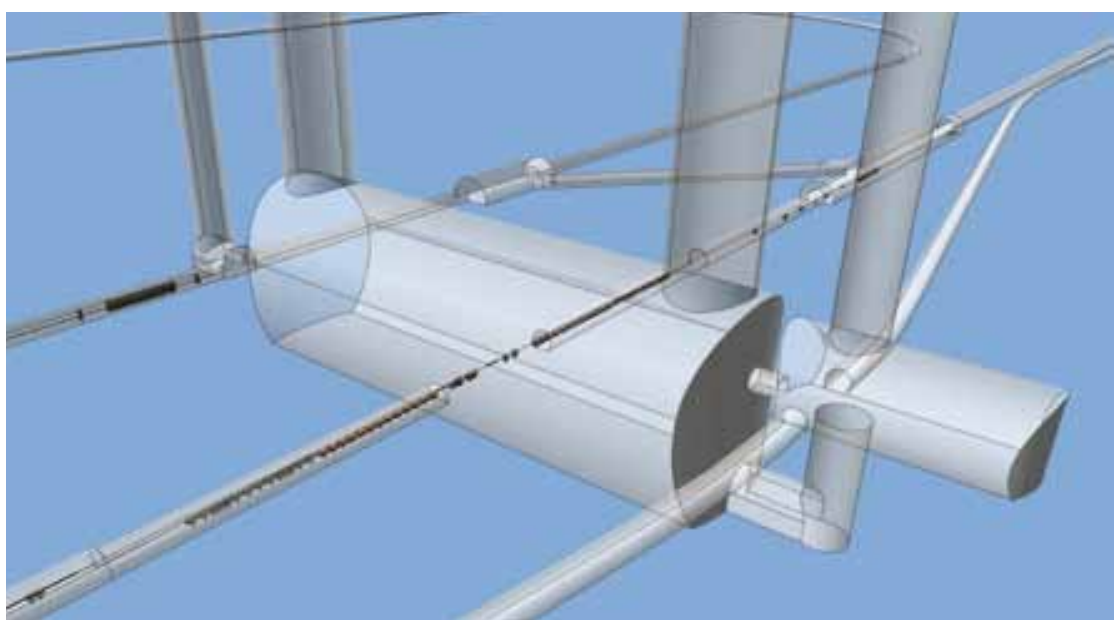




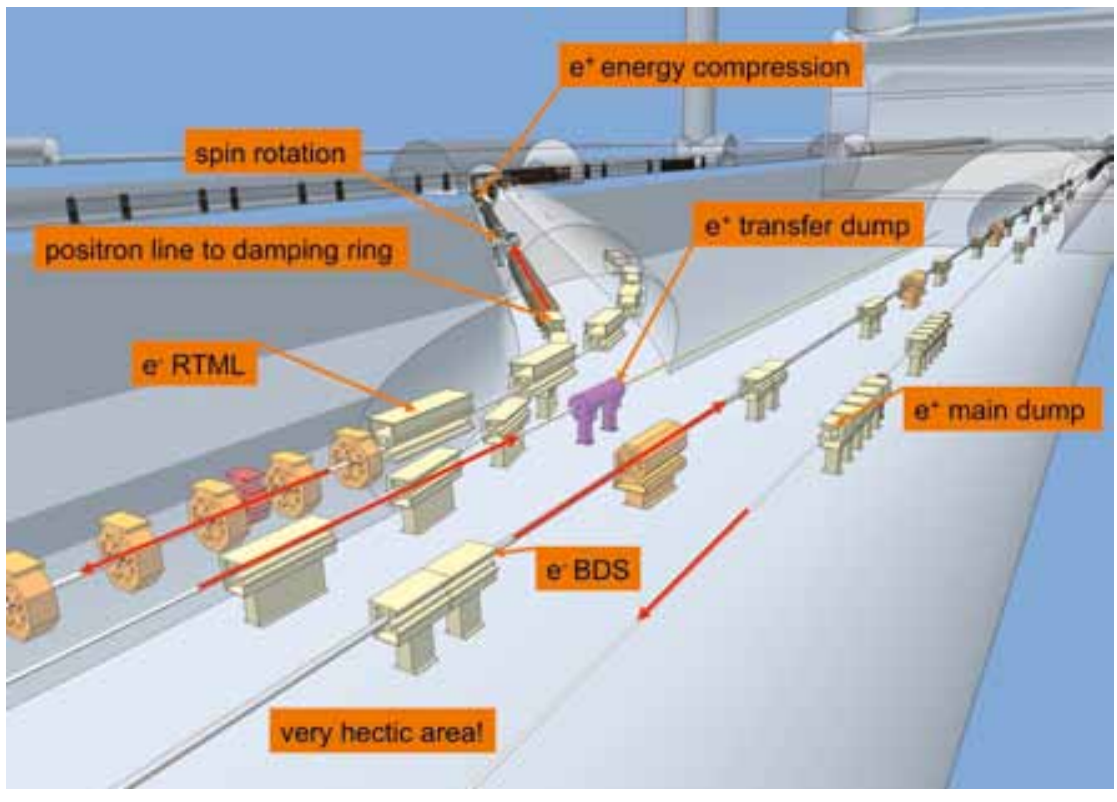

Figure 5.8 A detail of the Central Region tunnel showing typical beamline installation.

Figure 5.7 shows an enlarged depiction of the interaction region and adjacent damping ring and ring-to-main-linac connections to the beam delivery system enclosure. Figure 5.8 shows one of the more congested areas of the entire underground complex. This is a view looking towards the interaction region from the end of the electron main linac. At the right side of the drawing is the positron beam transfer line from the interaction region to the main positron absorber. To the left, the electron beam delivery system carries the electron beam to the interaction region. To the left of the electron beam, and floor-mounted, is the low-energy positron transfer line from the positron source to the damping ring. Finally, above the positron transfer line, the electron ring-to-main-linac transfer line from the damping ring to the start of the electron main linac is indicated positioned at ceiling height above the other beam lines. Red directional arrows indicate the direction of travel for each of the beam lines. A similar condition exists on the opposite side of the interaction region at the end of the positron main linac. These 3-D drawings are an essential tool for the conventional facilities effort. Work continues in all three regions to use the 3-D drawings to understand potential interferences, evaluate installation and maintenance techniques and develop workable design solutions for the entire range of underground enclosures from the simplest to the most complex. 


\subsection{LIFE SAFETY AND INFRASTRUCTURE SUPPORT}

While the underground tunnels, enclosures and caverns constitute the largest component of the conventional facilities design, the safety of personnel and the configuration of supporting utilities are also very important parts of the overall design. When the accelerator complex is operating, only a few underground areas are accessible to personnel. However, during initial installation and ongoing maintenance periods, there is likely to be a good deal of activity in many parts of the underground space. It is for these times that proper precautions must be taken to ensure the safety of workers underground in general. People's safety and stable machine operation also depend on a variety of utility support systems. An accelerator with the magnitude and complexity of the ILC requires a stable temperature environment, a process water system for component cooling and a high-voltage electrical distribution system to supply the power needed to operate the machine.
Figure 5.9 The main linac tunnel design has been modified from a two-tunnel to a single-tunnel scheme.

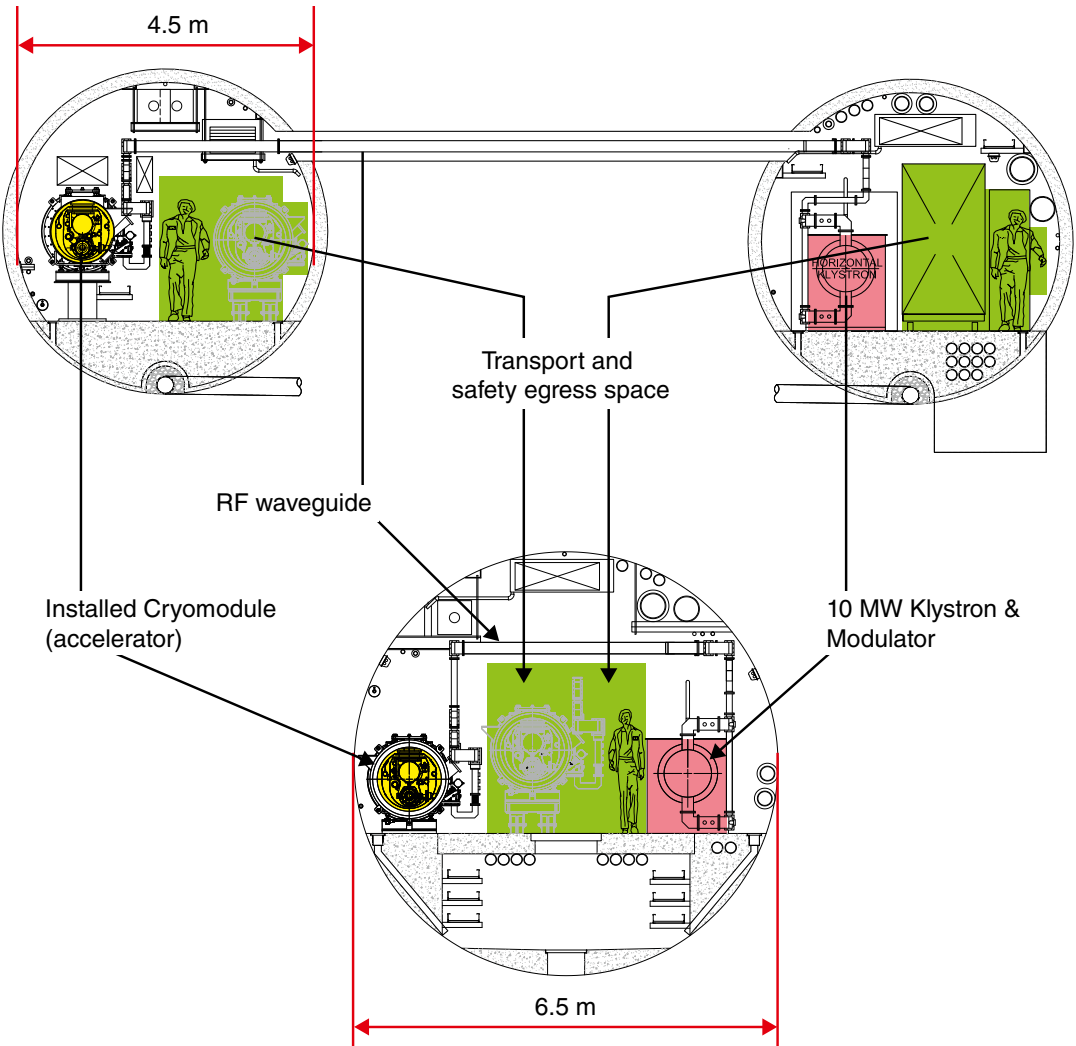

An important part of the Technical Design Phase was the decision to move from the Reference Design Report (RDR) twin-tunnel configuration to a single-tunnel configuration for the main linacs. The damping ring also has a single-tunnel configuration while the beam delivery systems retain an adjacent service tunnel configuration due to requirements for support equipment. Figure 5.9 illustrates the transition from the twin-tunnel RDR scheme for the main linacs to an RDR-type single-tunnel solution. Other solutions for a single tunnel have been developed based on different RF 
system configurations and local geologic conditions. But fundamental to all of these alternative single-tunnel solutions was the need to develop sensible life safety and egress solutions for a single-tunnel configuration that were compliant with prevailing codes and regulations. The twin-tunnel configuration allowed a fairly straightforward approach to life safety and egress. If a fire or other hazard occurred in one of the tunnels, the second tunnel could be isolated and used as the emergency escape route. With a single-tunnel configuration, the issue of emergency personnel egress had to be studied. Varying regulations in the three regional areas has led to two different solutions to the problem.

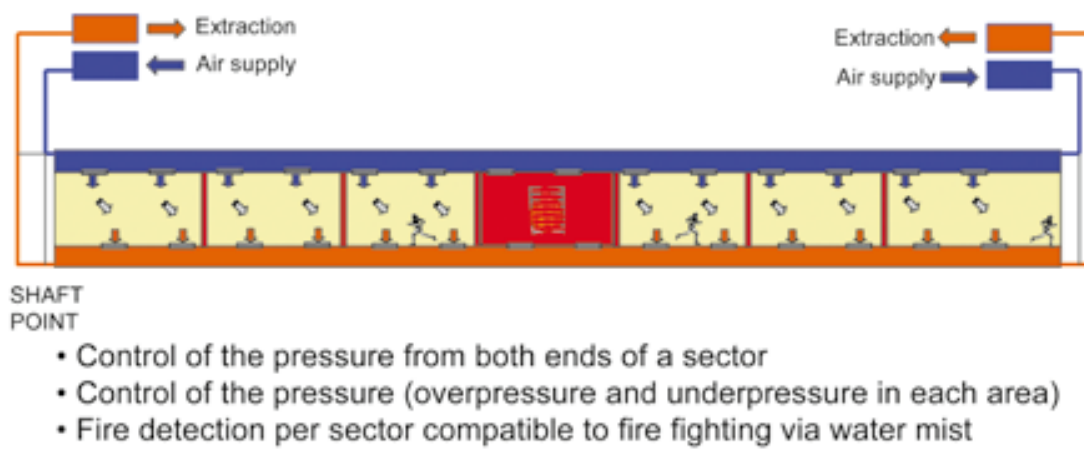

Figure 5.10 A scheme of the exit plan for the main linac tunnel if the ILC were built in the European region.

The Asian and European regions have both developed a similar approach to the life safety and egress issue. Their solution divides the single-tunnel portions of the main linacs and damping ring into 500-m increments or 'compartments' that are separated by fire walls and automatic fire doors that can isolate areas that may be involved in a fire or other incident. Figure 5.10 is a schematic drawing that shows how this compartmentalisation scheme can isolate a hazardous area. Another important part of this approach is the control of airflow in an emergency. As the drawing indicates, a continuous duct for air supply and extraction extends along the entire length of the single-tunnel enclosure (see previous tunnel cross-section drawings in section 5.1). Under normal operation, supply conditioned fresh air to the tunnel enclosure is returned for conditioning and air change through the extraction duct. In the event of a fire, the affected area can be sealed by firewalls and doors. Dampers will close in the supply duct, preventing fresh air from contributing to the fire and controlling smoke from permeating beyond the affected area. In this way the unaffected areas of the tunnel can be used for personnel egress to the surface and for emergency response personnel to access the affected area.

In the Americas region a different approach is required due to stipulations in the prevailing regulations for underground construction. Instead of dividing the underground space into small areas, the containment is based upon the enclosure of the highest-hazard areas with fire-rated walls. This highesthazard equipment is actually not the accelerator itself, but in some of the equipment required to support accelerator operation. Oil-filled electrical equipment, water pumps, motors and other utility equipment constitute the highest potential for fire. This equipment is located in the caverns at the base 
of the vertical access shafts located along the single-tunnel enclosures. In the Americas region solution, these local areas are isolated by fire-rated walls and doors, which leave the main tunnel enclosure available for personnel egress to the surface. It is required to have a fire-protected area of refuge at intervals of $1,200 \mathrm{~m}$ along the length of the single tunnel to provide an intermediate safe area for injured personnel or to await emergency response assistance. Figure 5.11 is a schematic drawing showing the areas of refuge with respect to the adjacent vertical shafts.

\section{Airflow during Emergency}

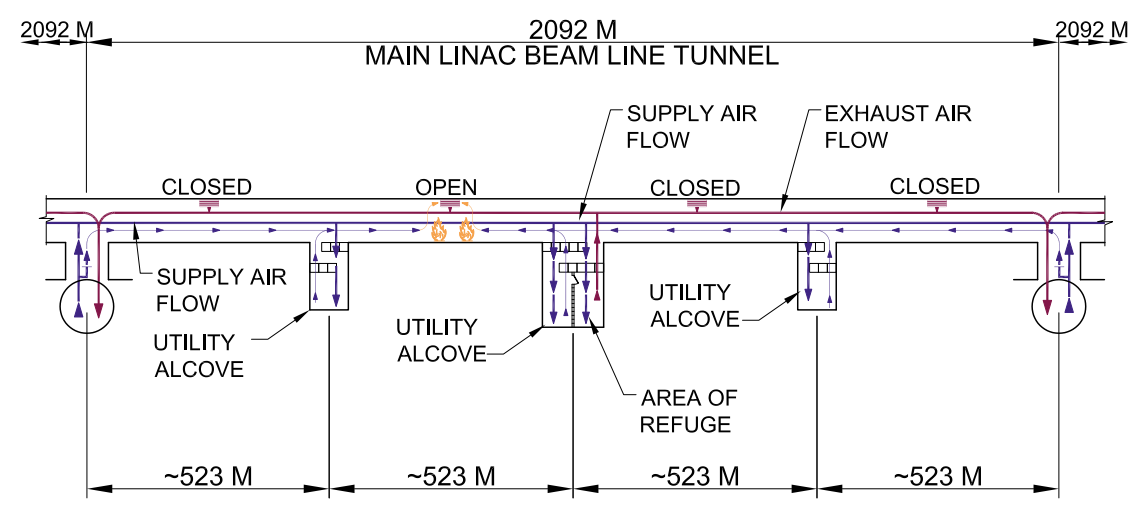

It is important to note that both approaches to life safety and egress are the direct result of extensive analysis of regional requirements supported in part by independent consultant review. Work to date has provided confidence that a single-tunnel solution can be constructed that will provide a safe working environment when people are underground performing machine installation and maintenance activities. When an actual site is selected for the project, the details of a final design solution can be developed in conjunction with local code requirements.

Another continuing focus within the conventional facilities design effort is the optimisation of the designs for the utility systems that support the accelerator operation. After the underground civil construction, the mechanical systems, including air handling and ventilation systems and process cooling water systems, are the second largest cost driver in the conventional facilities cost, with the process cooling water being the largest component. At the time of the RDR, the major portion of the process water system design was completed in the Americas region and adopted for use in all three regions. During the first two years of the Technical Design Phase, the Americas regional team completed a formal value engineering review of the process water system. The value engineering process involves identifying major drivers to the existing design and identifying alternatives for evaluation and possible inclusion into the design to improve efficiency and reduce costs. During this process it was determined that the process cooling water design developed as the reference design was based on criteria provided independently from all of the contributing parts of the ILC machine. In many cases these criteria were 
optimised for each specific part of the machine, or area system, which resulted in a varied set of requirements and produced a very complicated and expensive process water system design. One of the most important outcomes of the value engineering review was to identify specific extreme criteria that were complicating the process water design and work with the various area systems to relax some of the more stringent criteria. In doing so, a more uniform and simplified process water system design was achieved and costs were reduced.

In the conventional facilities work that led to the development of the ILC Reference Design Report, no specific site investigation was included. Instead, each region identified a generic sample site and used a common overall machine layout and underground configuration on which to base the initial cost estimate. During the current Technical Design Phase, preliminary site investigation has been started in varying degrees and in all regions. In the Asian region, two specific sites have been identified in Japan as possible locations to be considered for the construction of the ILC. In the European region, the sample site location at the CERN laboratory in Switzerland as well as a possible site in Dubna, Russia, near the Joint Institute for Nuclear Research are undergoing preliminary site investigation as well. In the Americas region, although specific site investigation has not begun, studies for surface building arrangement at shaft locations have been completed. In the Asian region the down-selection to two specific sites in Japan as candidate sites for the location of the ILC project marks the most mature of the site-specific investigation efforts. The Asian conventional facilities and siting group has been working jointly with the Japanese Advanced Accelerator Association Promoting Science \& Technology (AAA) in the development of preliminary design for these sites. The member corporations of the AAA have a great deal of cumulative experience in the construction of tunnels in Japan, primarily for car and rail traffic. Both of the selected sites, one located in the northern part of Japan and one located in the southern region, are in mountainous areas and as such present unique challenges for the construction of large underground complexes.

\section{$5 \cdot 3$ SITE-SPECIFIC DESIGN EFFORTS}

Figure 5.12 Schematic of a mountainous region tunnel provided by the Japanese Advanced Accelerator Association Promoting Science \& Technology. 
Figure 5.12 is a schematic transverse section of one of the selected sites; both sites have a similar mountainous profile. In a mountainous region, vertical shaft access to the surface and the corresponding surface facilities are in most cases unworkable solutions, so gently inclined horizontal tunnels will be used to provide access to most parts of the underground accelerator complex. The geology for both of the sites being considered in Japan is primarily granite, which affords the possibility of tunnel construction using either tunnel boring machines or the drill and blast method.

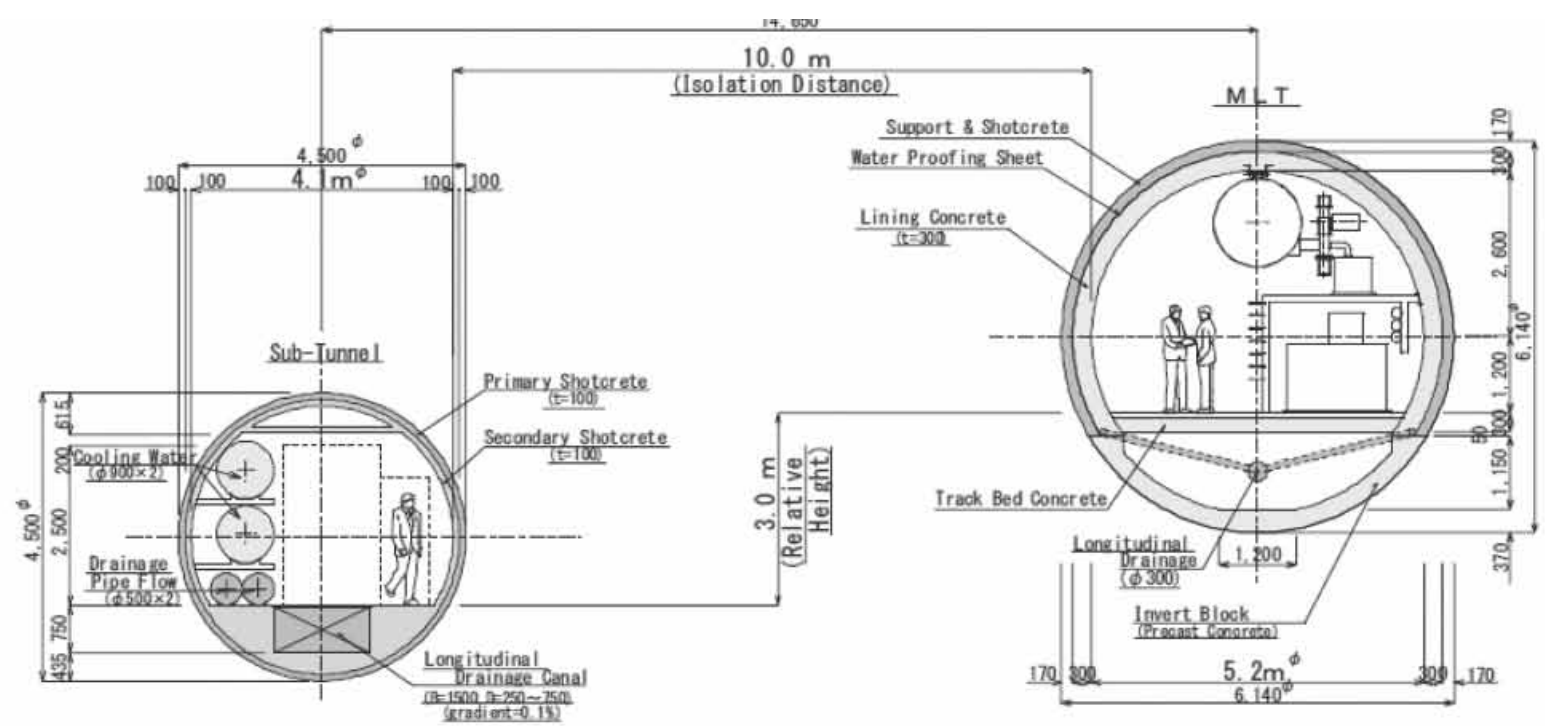

(section)

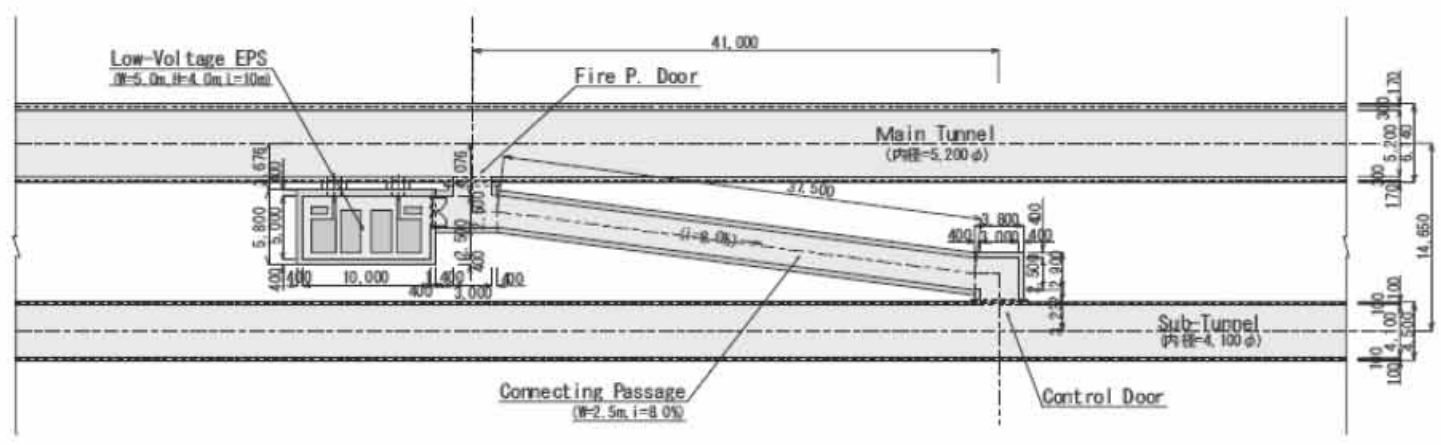

(plan)

Figure 5.13 A cross-section of a mountainous region tunnel provided by the Japanese Advanced Accelerator Association Promoting Science \& Technology.

A unique aspect of the design approach in Japan's mountainous regions is the construction of a smaller-diameter pilot tunnel ahead of and slightly below the level of the main accelerator tunnel. This approach is used for most traffic tunnels in Japan and the benefit of the pilot tunnel is two-fold. First, water inflow in this type of construction is expected and the pilot tunnel provides a means to dewater the main tunnel during both construction and operational use. Second, the pilot tunnel can also be used 
as an additional means of egress in the event of an emergency or hazardous event. Figure 5.13 shows a typical cross-section through the main linac tunnel with the lower pilot tunnel as well as a plan view of the two-tunnel configuration with a connecting passage between the two tunnels. In the case of the ILC, the pilot tunnel can also be used for support equipment in addition to ground water control and egress. While mountainous sites require most of the accelerator complex to be housed in underground tunnels and caverns, some major support installations will still be required to be located on the surface. These installations will likely be adjacent to one or more of the adits of the horizontal access tunnels. Figure 5.14 is a schematic layout for the main substation that will supply the electrical power needed to support the accelerator complex. This substation layout is likely to be applicable to any site being considered to host the ILC project.

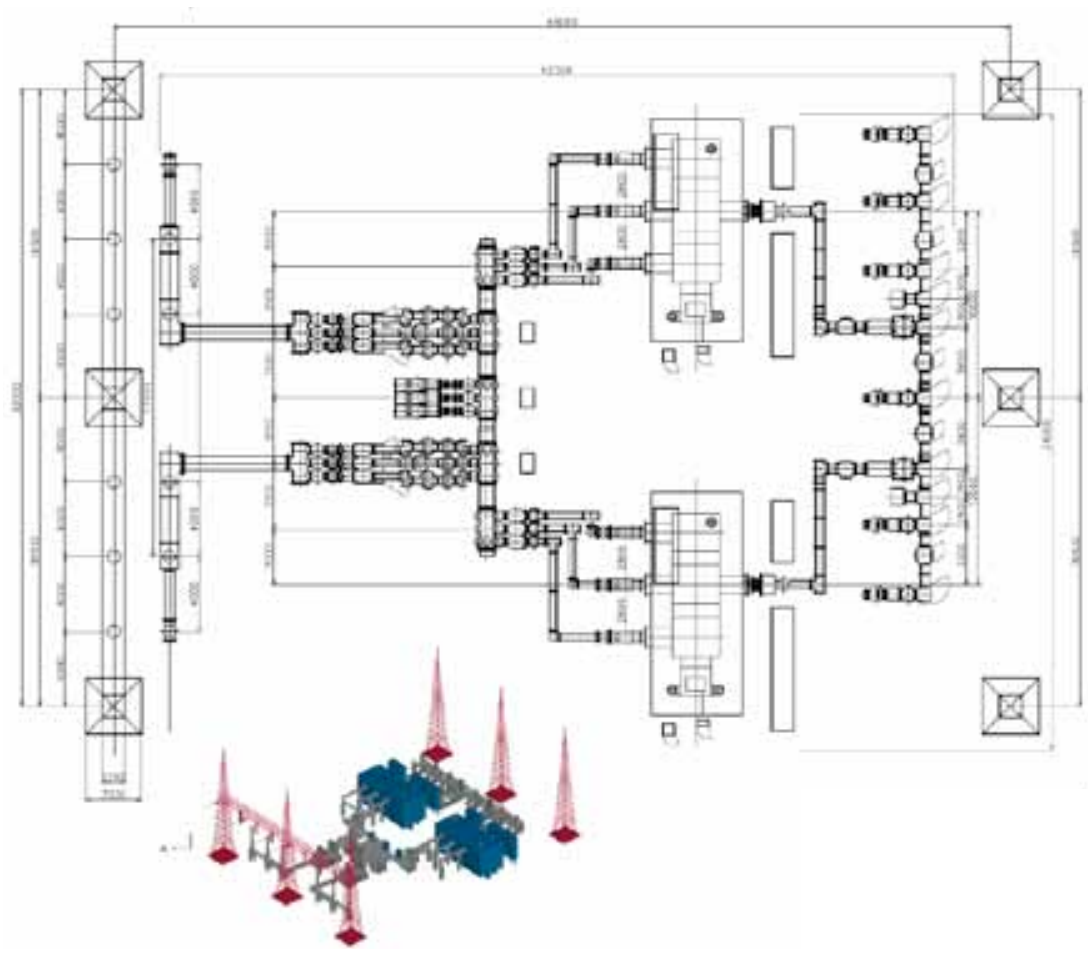

Figure 5.14 Diagram of the electrical utility master substation.

The European region sample site developed for the Reference Design Report was also a somewhat mountainous region located near CERN, although it was not subject to the constraints of the more mountainous Japanese sites. Vertical shafts will still be used to access the underground complex and surface structures at the shaft locations are still being considered. Specific site investigation has not yet begun with the exception of the development of preliminary geologic profiles along the alignment of accelerator complex. 
Figure 5.15 shows a schematic transverse section of the proposed alignment of the European sample site. The geology at this site is primarily a sandstone/ molasse rock, which favours a fully lined tunnel construction method with a tunnel boring machine.

Another European site that is being considered is located in a rural area of the Russian Federation in the northern Moscow region near the town of Dubna and the Joint Institute for Nuclear Research. Figure 5.16 shows the initial proposed alignment north of the city of Taldom. While the actual design is at a very preliminary stage, some geologic site characterisation and soil boring work has been completed that verifies the consideration of this site and the potential for future conceptual design work. Figure 5.17 shows a schematic transverse section of the proposed alignment of the Dubna site. Unlike the deep tunnel designs that are currently being considered in Japan, at CERN and in the Americas region, the Dubna site design would incorporate a relatively shallow bored tunnel configuration with surface support buildings. The site is relatively uniform in surface elevation compared to the Asian and CERN sites and the tunnel would be bored in an impermeable soil-based stratum rather than in the deeper rock.

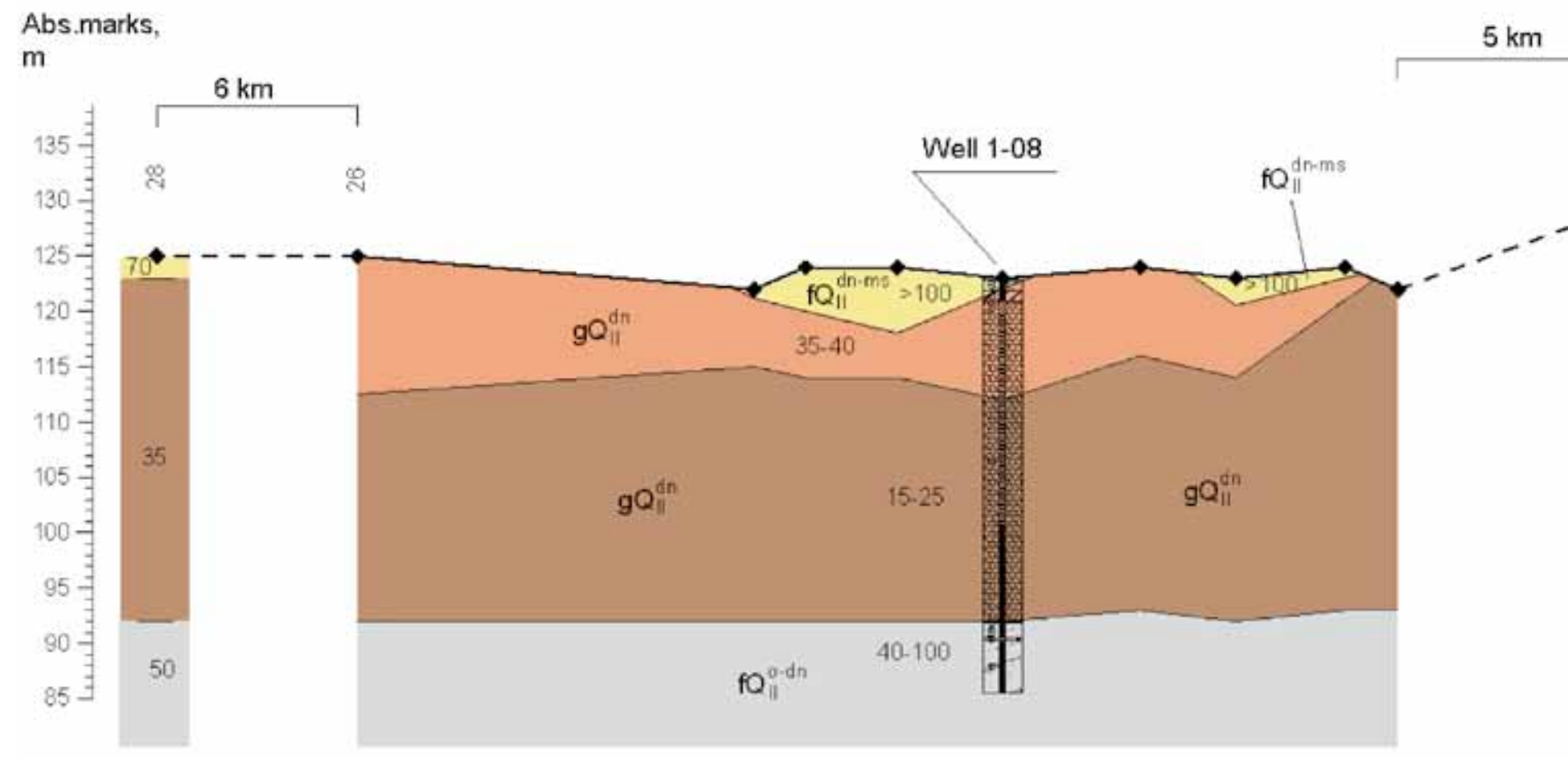




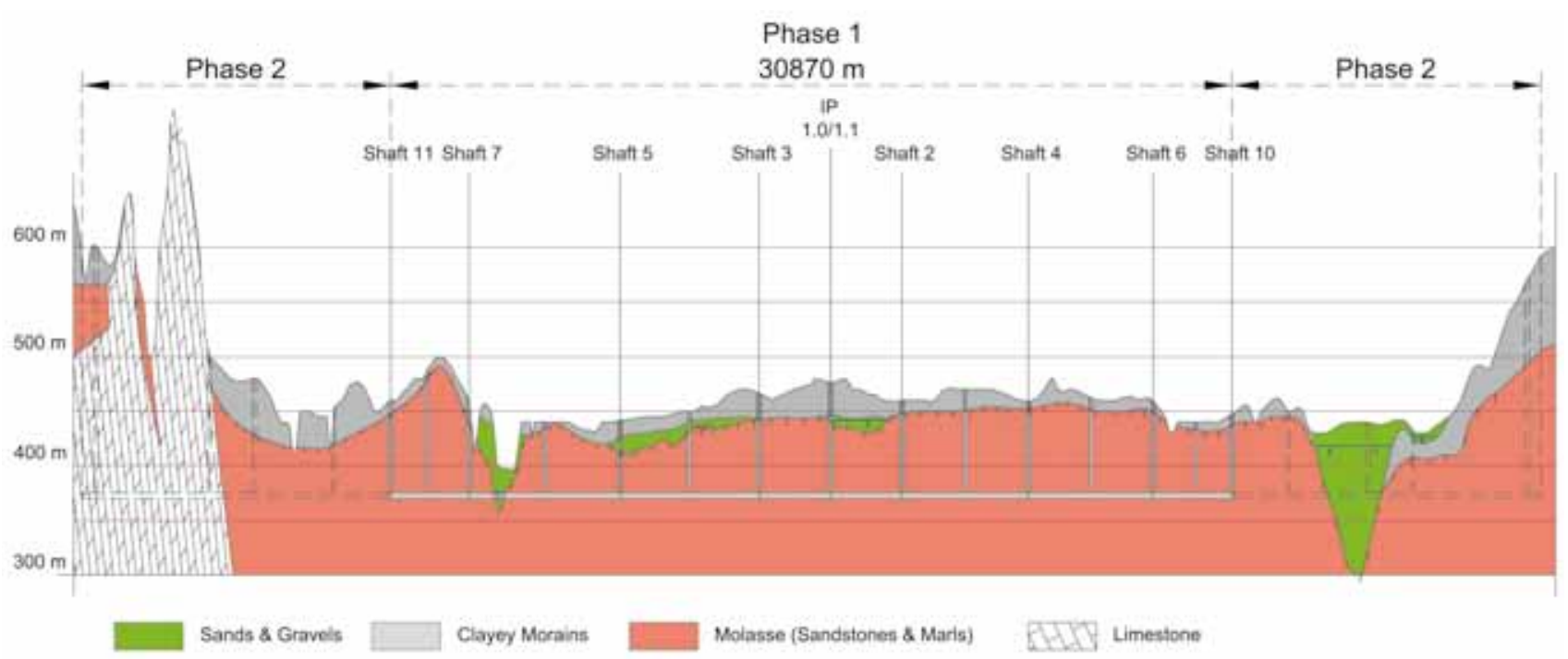

Figure 5.15 Tunnel geology of the CERN region.

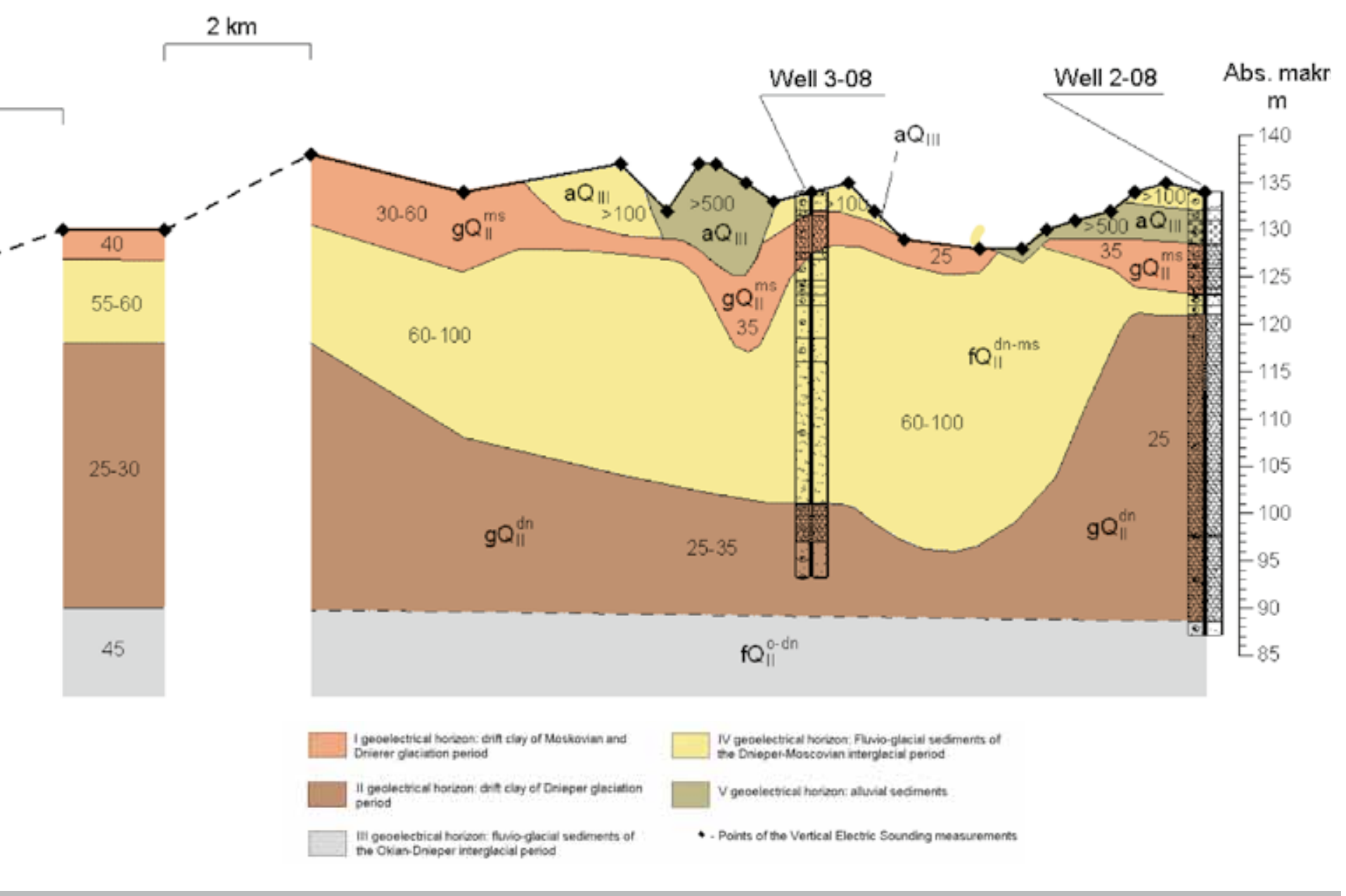


Figure 5.16 The linear collider alignment near Dubna in the Moscow, Russia region.

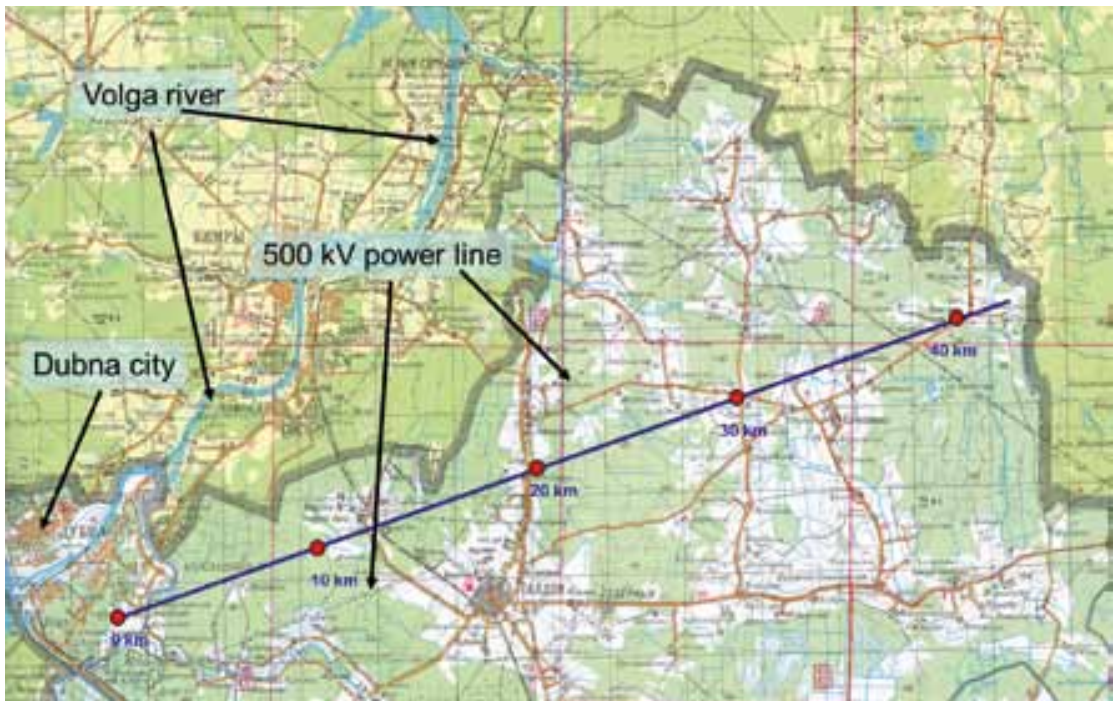

Figure 5.18 is taken from a cost study that was completed in the Americas region that compared deep-, shallow-bored and cut-and-cover site conditions and tunnel configurations. This schematic perspective view shows a possible configuration for the conditions found at the Dubna site.

In the Americas region the sample site that was used for the Reference Design Report was a site in northeastern Illinois near Fermilab. This site is also relatively uniform in elevation along the machine alignment. However, due to the favourable geologic conditions of the limestone bedrock, a deeptunnel configuration is the preferred solution for this site. The Americas region has continued to be a full partner in the global effort to develop an updated baseline with respect to the previous reference design. While the preliminary design is well developed, there has been limited actual site investigation up to this point in the Technical Design Phase. There is, however, a great deal of local experience in tunnel construction in the limestone bedrock as a result of the Deep Tunnel Wastewater project recently constructed in the greater metropolitan Chicago area. Experience gained from this project has been incorporated into the design for the Americas sample site. 


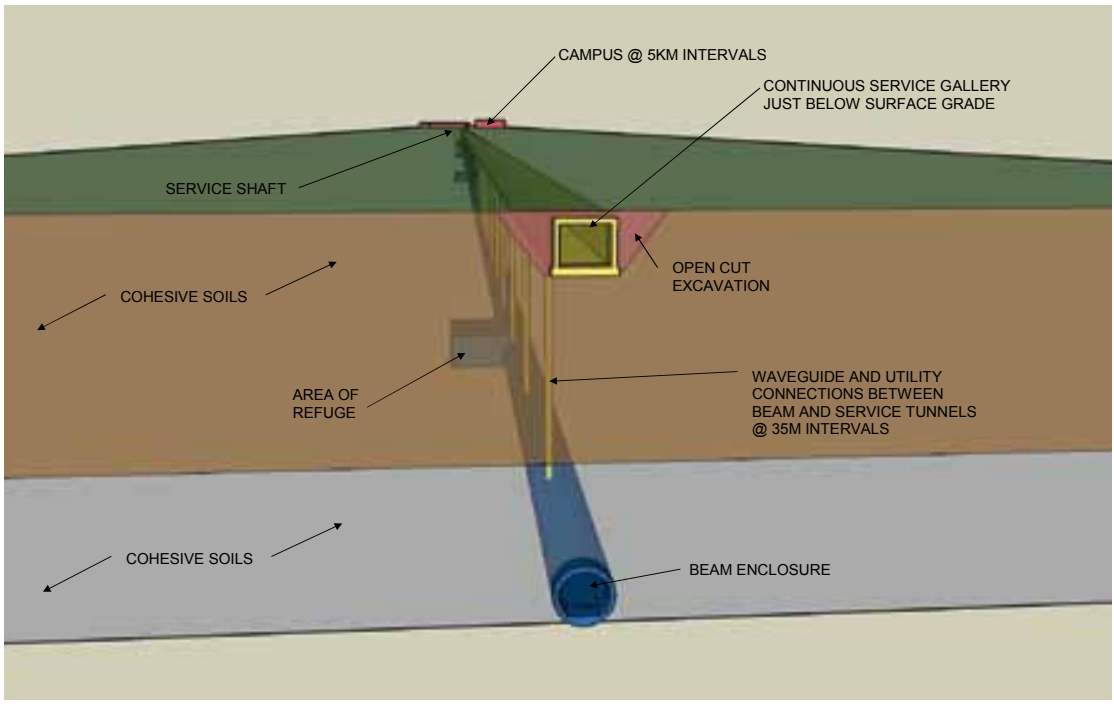

Figure 5.18 Tunnel schematic cross-section near Dubna in the Moscow, Russia region.

Figure 5.19 shows a schematic transverse section along the proposed alignment for the Americas sample site. The dolomite limestone bedrock is a very uniform geologic layer and is also relatively dry, which lends itself to the use of tunnel boring machines and drill-and-blast methods for the construction of underground tunnels and caverns.

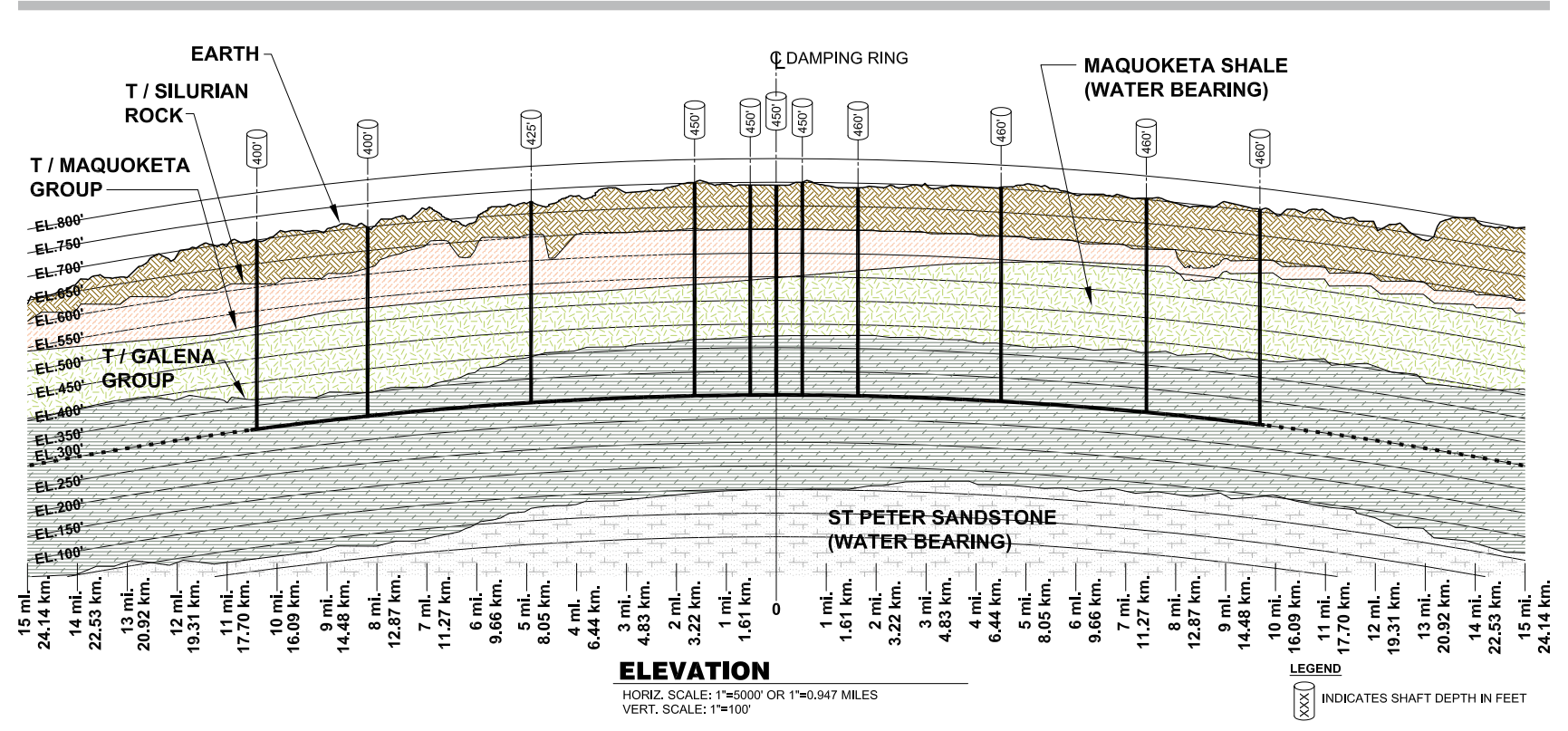

Figure 5.19 Tunnel geology of the Americas region sample site. 
A schematic perspective view of the single deep-tunnel solution, also taken from the Americas tunnel configuration study, is shown in Figure 5.20. The uniform elevation of the Americas region sample site allows for the construction of surface buildings for utilities and other equipment at and around each vertical shaft location. Variations on the preliminary work for these surface campuses will necessarily depend on exact site selection, which may occur at a later date. However, conceptual work has been completed to develop general schemes and land requirements should this site be considered in the future. Figure 5.21 shows a plan view of one of the surface campus layouts at a vertical access shaft. Included in this layout is space for cryogenic and high-level RF accelerator support equipment as well as process water cooling and conventional electrical power equipment. Figure 5.22 shows the same layout in isometric form superimposed on a generic aerial photograph.

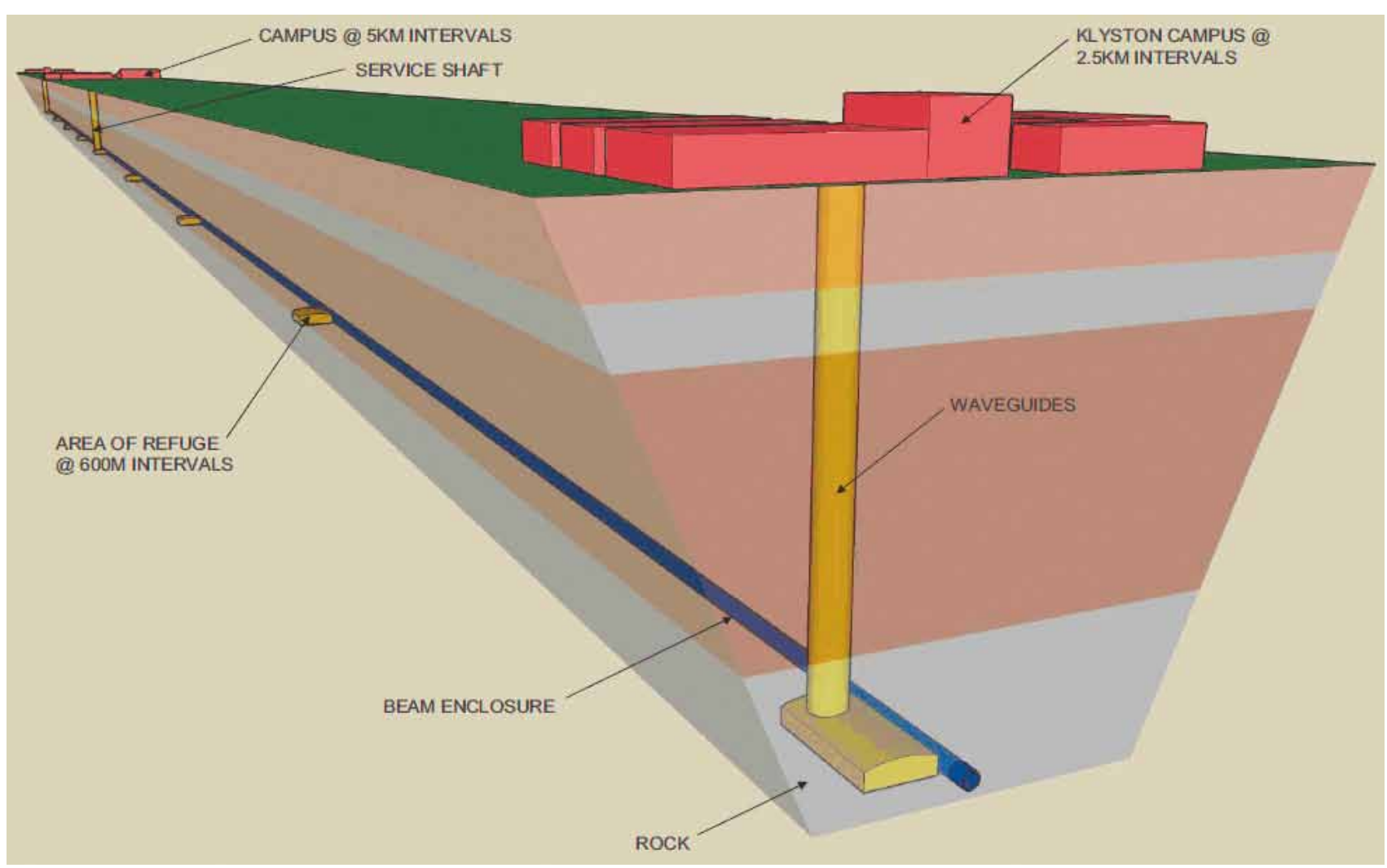

Figure 5.20 Tunnel cross-section schematic for the Americas region.

Variations on the preliminary work for these surface campuses will necessarily depend on exact site selection, which may occur at a later date. However, conceptual work has been completed to develop general schemes and land requirements should this site be considered in the future. Figure 5.21 shows a plan view of one of the surface campus layouts at a vertical access shaft. Included in this layout is space for cryogenic and highlevel RF accelerator support equipment as well as process water cooling and conventional electrical power equipment. Figure 5.22 shows the same layout in isometric form superimposed on a generic aerial photograph. 


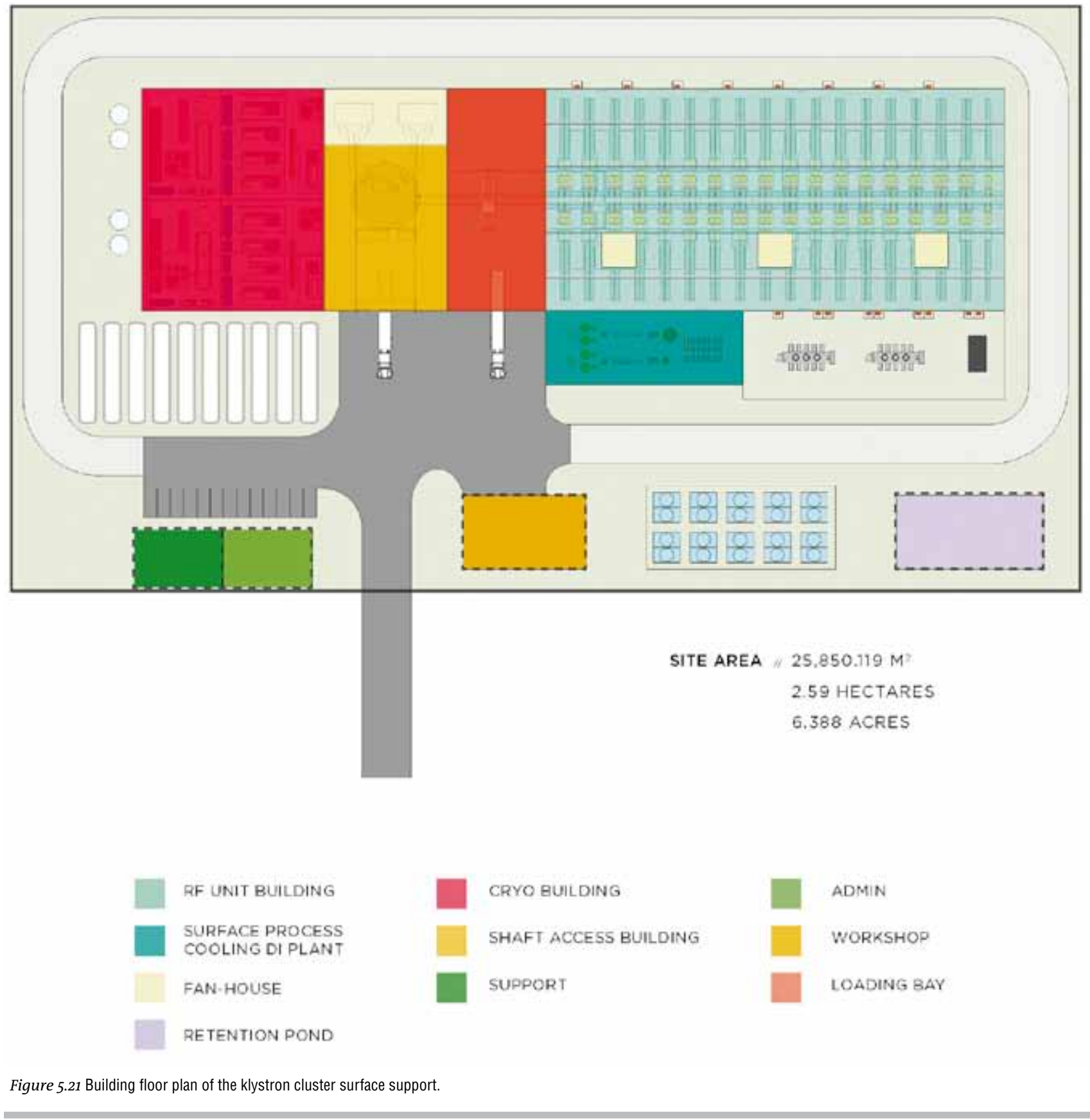




\section{CONVENTIONAL FACILITIES AND SITING}

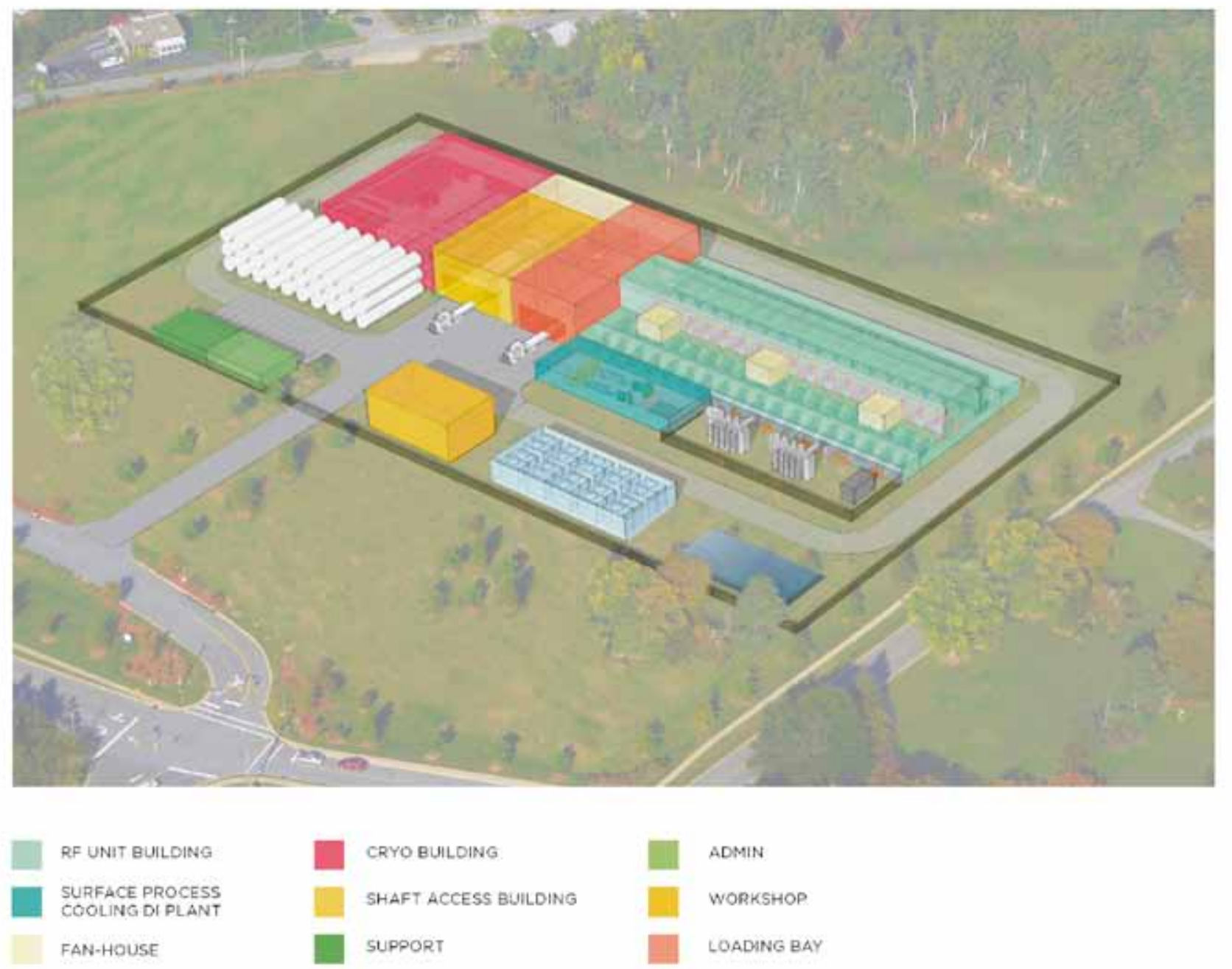

Figure 5.22 Aerial view of the klystron cluster surface support building.

The actual site selection process for the ILC project will begin in the coming years. However, preliminary work to investigate the potential of various sites located in all parts of the world provides a global approach to understanding the relative costs for each of the different design approaches and conditions that are likely to be encountered for each of the various sites being considered. 
CONVENTIONAL FACILITIES AND SITING 


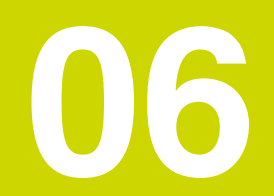

6.1 A FOCUS ON MASS

PRODUCTION AND COST

6.2 CONSOLIDATING R\&D

6.3 THE PROJECT

IMPLEMENTATION PLAN

6.4 BEYOND THE TECHNICAL

DESIGN REPORT

\section{TOWARDS THE TECHNICAL DESIGN REPORT}


Phase 2 of the Technical Design Phase (TDP) will see a major focus on costeffective mass-production scenarios for the superconducting radiofrequency (SCRF) cavity and cryomodule systems. As the primary cost driver for the ILC, establishing a defendable and realistic cost for the industrial manufacture of some 2,00o cryomodules will be by far the most critical issue facing the Global Design Effort (GDE) as it prepares for the Technical Design Report (TDR) at the end of 2012.

In order to establish an internationally agreed cost estimate for the cryomodules, several key themes will need to be developed:

- Cavity and cryomodule gradient specification and projected production yield

- Flexible technical specification based on a plug compatibility concept (interface definition and specifications)

- Production process and industrialisation models

- Models for international cooperation including in-kind contributions

Several issues go beyond just production engineering. For example, understanding the need to maintain flexibility in the industrialisation models to support in-kind contributions is seen as a high priority. As a result, understanding how the production of cryomodules might be divided among contributing nations (or regions) must be factored into the production models. At the same time, a strong emphasis on mass-production techniques must be adopted to maximise the expected significant cost-reduction factor associated with large-volume production. A further desire is to maintain as far as possible global competition between possible vendors, with a view to reduced cost to the ILC project. Last (but not least), a cost-effective approach to risk mitigation needs to be developed, one that does not place too stringent performance guarantees on vendors that would likely increase the costs.

Early in the Technical Design Phase, the concept of plug-compatible design was introduced for the components of the cryomodule (specifically the cavity package). A set of interface definitions have been internationally agreed upon that, in principle, would allow alternative component designs to be assembled in the same cryomodule. During the current R\&D phase, plug compatibility has allowed parallel development of technologies such as cavities, tuners, and high-power couplers. This has allowed regional teams to develop in-house design expertise, effectively promoting local innovation, while maintaining global cooperation and sharing advanced technology.

The concept of plug compatibility can now be directly translated to the massproduction models (construction), where it will:

- support competition between multiple suppliers, promoting cost reduction, while allowing variants within a common design envelope;

- allow for multiple (in-kind) contributors, each of which may arrive at a different cost-optimised design, maximising the benefit of local industrial capability and experience;

- encourage intellectual interest from each contributor to promote regional, national or institutional centres for integration and test of the cryomodule or any of its subcomponent packages.
6.1 A FOCUS ON MASS PRODUCTION AND COST 
The published cost estimate in the RDR was firmly based on the original European-based industrial studies performed for the TESLA proposal in 2000 [6-1]. These studies were based on a single-vendor model for the production of 20,000 cavities (about 1,700 cryomodules) in a five-year time scale (two years for setup and pre-production, three years for peak production). While these studies represent a solid basis for one particular industrial model, it is now necessary that the GDE produce its own updated estimate given the progress in the last ten years. Specifically,

- The last ten years of R\&D have seen the establishment of SCRF technology in both the Americas and Asian regions, and a Europeancentric estimate must now be reviewed.

- The governance issues mentioned above (in-kind contributions leading to distributed mass-production) should be considered and the possible impact on the costs quantified. This suggests moving away from the single-vendor model used in the RDR estimate (but does not necessarily exclude it).

- Production risk should be more carefully considered, which would also tend towards a multiple-vendor model.

Finally, the recent experience of the European X-ray Free-Electron Laser (or European XFEL) mass-production will need to be factored in, as this will be the largest production and deployment of the technology within the TDR timescale. However, at approximately $5 \%$ of the ILC production requirement, care needs to be taken in extrapolating these costs.

Many of the key issues mentioned above are driven primarily by politics, which is at this stage difficult to predict. Exactly how the mass-production of cryomodules will be divided amongst possible contributors (in some form of in-kind model) will only be exactly known at the time of approval. However, the GDE needs to be prepared to answer the inevitable questions that will be asked, which will require the development of more than one massproduction model (and ultimately cost estimate). Therefore it is necessary to develop cost estimates in all three regions based on a limited set of in-kind contribution scenarios. Approaches to mass-production in each region will need to be studied that take into account the differences in local industry technical expertise, markets and cost. This will likely result in different technical approaches to mass-production, each cost-optimised for the specific scenarios considered and the local regional industrial environment.

In developing the models and associated cost estimates it will be necessary to

- review the existing manufacturing process, and understand the primary cost-drivers (information from the European XFEL will be extremely useful in this respect),

- look for the most cost-effective production technology and approach towards aggressive cost-reduction for large-scale mass production; this includes R\&D towards cost-effective production techniques and possible modifications to the cryomodule design (design for manufacture), and

- communicate with regional industry and laboratories, with a view to developing cost-effective manufacturing, testing and quality control models.

The role of the large regional laboratories is seen as central to cost-effective mass production. In particular, these labs would provide testing and quality control facilities, and possibly act as the primary integrator of sub-components delivered by industry. Such a model is similar to the approach adopted by 
CERN for the LHC dipole mass production. Performance guarantees would then be the responsibility of the central labs; this is seen as key to reducing to a minimum vendor risk, and thereby reducing the costs. The central labs - acting as regional production centres - are then directly responsible for delivering complete cryomodules (for example) to the future ILC lab. While other massproduction models exist, the lab-based regional centre model is clearly one that would fit naturally with a globally distributed cryomodule production, driven by in-kind contributions. For all models considered, an important aspect is to maintain global market competition between vendors. A primary GDE goal for the Technical Design Phase is the development of industrial capability in all three regions, but this should be seen as developing a global competition base, rather than guaranteeing regionally centred contracts to local industries.

To facilitate the development of the mass-production models, and to prepare the way for cost estimates based on industrial quotes, a series of visits to established cavity and cryomodule manufacturers in all three regions was made in 2009, primarily to establish contact and to gauge first-hand the scale of the production facilities. This was followed by an industrial workshop on SCRF cavity industrialisation, held in Kyoto in 2010 [6-2]. The GDE now plans to make a second visit to the vendors in 2011, specifically to begin discussions on mass-production and ultimately producing tentative cost-estimates for cavity production based on a well-defined process specification ('build to print'). The industrial feedback received will then be factored into GDE updated cost estimate for the TDR.

Following the completion of the Reference Design Report in 2007, the focus of the ILC Global Design Effort shifted to component and system development and performance demonstrations. In keeping with a worldwide three-region strategy, the foremost principle of the GDE, the work has been started and carried out evenly in each region by the global partnership team. A summary $\mathrm{R} \& \mathrm{D}$ plan that explains the effort, including tables showing where the work is done, has been kept updated and published semi-annually since mid-2008.

The primary objective of the R\&D is to reduce technical risk and find ways to reduce project cost. Also, since the timescale of ILC construction is unknown, the GDE promotes and is constantly in search of alternate strategies for various aspects of the design, including subsystems and components. Through that, the project is kept updated and does not become frozen as can often happen in long-term high-technology projects.

During the second half of the Technical Design Phase the GDE will take stock of risk-reduction and cost-saving R\&D work and assess what is useful and appropriate for inclusion in the Technical Design Report to be published in 2012. In this section, we summarise these efforts and outline what may be included in the TDR. In some cases, work will continue beyond the publication of the TDR and this is also outlined.

GDE Research and Development work can be divided into three categories: high-technology scientific and engineering studies, beam-based studies and component and sub-system design development. 


\subsubsection{High-technology scientific and engineering studies aimed at expanding the state-of-the-art frontier}

Superconducting radiofrequency process and test infrastructure in several institutions was commissioned successfully during the Technical Design Phase. To provide the greatest return, these facilities were designed and constructed to address a variety of challenges inherent in the emergent technology. While each one is built to satisfy ILC project-specific R\&D goals, each also includes enough flexibility to serve the goals of other projects. This strategy, applied globally, resulted in both redundancy and diversity.

Research and development goals (chapter 2) are arranged to focus attention on three aspects of the technology: 1) the basic nine-cell niobium sheet metal cavity, 2) the cryomodule assembly and 3) the operation of a system based on several cryomodules at full ILC main-linac beam intensity. A fourth focus of the R\&D is on mass-production aspects of the cavities and cryomodules. For the latter, each of the three regions adopted a different approach - production of roughly 600 cavities for the European XFEL, development of a pilot plant for industrialisation studies in Asia, and studies of longer-term improvements to the basic process in America. During the Technical Design Phase, we will see results from each of the four focal points. Each of these will be included in the TDR, especially the analysis of mass-production techniques.

Notwithstanding this global progress, much of the infrastructure has just been commissioned and full system tests, especially, will not be started until after 2012. While we expect the primary objectives of linac system testing to be achieved in time for the TDR, the potential of the multi-cryomodule high-current test linacs to demonstrate new cost-saving designs will not be realised by then. The highest post-2012 priority for these installations will be to subject the new technology to a value engineering cycle, together with regional industrial partners. A component of this effort will be in support of the $1 \mathrm{TeV}$ energy upgrade for the ILC. We expect $1 \mathrm{TeV}$ upgrade-related R\&D to address issues in each of the four focal points, and hope for performance (and cost) breakthroughs in cavity gradient, cryomodule and coupler design, system integration and industrialisation and mass-production.

\subsubsection{Beam-based studies based at ILC purpose-built test facilities} Three beam test facilities were constructed with ILC beam parameters in mind and presently devote substantial operations time to ILC R\&D. These are CesrTA (Cornell, Americas) for studies of the electron-cloud coherent instability, ATF/ATF2 (KEK, Asia) for studies of precision beam optics and tuning, and TTF/FLASH (DESY, Europe) for studies of high-current superconducting linac operation. It is fitting for investments of this size that the primary goals of each test facility have very little overlap with the goals of the other two. At each one, beam studies are carried by a global collaboration. Initial results from each - electron cloud mitigation strategies from CesrTA, low-emittance tuning from ATF, and full beam current tuning from FLASH operations - will be a highlight of the TDR. It is not expected, however, that the full suite of studies will be complete by the end of 2012; the work will continue beyond then. 
Each of the ILC test facilities has unique capabilities and will be useful for accelerator science and technology development well beyond the ILC. This is particularly true for ATF/ATF2, the only accelerator test facility built to study manipulation of ultra-low-emittance beams. Beyond the publication of the TDR, ATF/ATF2 will be valuable for development of precision magnets, instrumentation and alignment technology, coupled with development of iterative beam tuning procedures. TTF/FLASH studies are closely linked to preparations for the operation of the European XFEL, which should begin in 2014. Beam-based feedback loops, especially those intended for control of SCRF cavity fields, will continue to be a critical focus at FLASH because the long bunch trains enable precision performance and stability.

\subsubsection{Component and sub-system design development}

Through the development of the Reference Design Report from 2005 to 2007 and during the Technical Design Phase, development work on the baseline design (and alternate designs), has been strongly supported by the GDE. Examples of this include the polarised electron source laser and gun system, the positron source target and matching device, the damping ring fast injection and extraction kicker, the linac Marx modulator (alternate), the beam delivery and machine-detector interface superconducting final doublet magnet and the conventional facilities and siting development for sites with varied topography. At present, midway through the TDP, plans for reviewing and evaluating the work done are in progress. Criteria to be applied are performance, anticipated cost, needs and plans for additional development and interface to various systems.

For the TDR, technical subsystem choices made through the review process will reflect the state-of-the-art ILC accelerator R\&D. In some cases, especially with projects that are close to completion, selections made for the technical design will assume the work to be successful. In addition, to make sure the design is kept dynamic and flexible following the TDR, we will promote work on new and promising technology and develop links to the teams doing that work. One way that will be done is through the collaboration with the CLIC study group, centred at CERN. This group has chosen to work on less well established technologies with tighter tolerances and that require more elaborate demonstration schemes. Nevertheless, we expect their design report, scheduled to be published around 2016, to include significant results that can be adapted to the ILC design. 
6.3 The Project IMPLEMENTATION PLAN
While the technical information relating to the ILC will be detailed in the Technical Design Report, there is additional information that will be useful to any collaboration member considering a possible bid to host. The Project Implementation Plan (PIP) will seek to provide, at an outline level, this kind of non-technical information emphasising areas where the GDE could reasonably be expected to possess an informed opinion. Examples include such topics as technical requirements for sites or in-kind contribution models. At this time there is no requirement for a highly detailed proposal, which would rapidly become out of date. Thus the PIP has the goal of establishing a general framework for considerations that should prove more enduring. The PIP will be produced on the same schedule as the TDR.

Many aspects of project implementation start from the basic project organisation, and the GDE will produce governance and funding recommendations in the PIP. This will be a summary of a more detailed GDE report on this subject. Specific proposals for organisation charts and the like are not warranted at this time. That will depend on the ultimate project management team. A synopsis of the project team and member state roles and responsibilities will be given in the PIP.

A sufficient number of large multinational projects have been completed to date that there seems to be a tacit understanding on the role of a host state, so this should not be too controversial. For example, land acquisition and services to the site boundary should not be a project cost. Civil construction and on-site utilities, which is part of the construction project, are generally accepted be a host responsibility. The host state must also agree to certain legal and quasi-legal conditions such as international access. The PIP will review the anticipated host state responsibilities.

In the absence of any additional information, then, a project schedule based on an LHC-like installation effort for the main linac recommends itself. Some progress in this direction was made at the ALCPGog workshop. This in turn would establish tunneling requirements. This input together with a 'traditional' start on the low-energy systems will provide sufficient information to develop a nominal project schedule. We intend to develop a crude high-level resource-loaded schedule based on the Technical Design Phase 2 cost estimate. This will provide guidance as to the natural project funding profile and will be part of the PIP.

Most scenarios involve substantial in-kind contributions, and outlining the appropriate interface points in the PIP would serve to furnish examples of the kind of technical contributions that collaboration members could be expected to provide. The exact details of member state contributions will, of course, only be determined during the final project negotiations. The use of in-kind contributions also implies that there will be an outsourcing of technical design and associated quality assurance activities. The PIP will outline the suggested respective roles of the project and the member state collaborators in this regard.

While the design of the ILC is not site-specific, there are requirements for the site that will not be expected to change significantly. The PIP will describe the major site requirements such as footprint, power needs, tunnel penetrations, 
central campus layout and so on. The GDE will provide different technical solutions to enable different site topographies to be considered. We expect the final ILC design to be site dependent to some degree. The actual siteselection process will be specified by the ILC Steering Committee. We do expect to include a summary in the PIP for the sake of completeness.

We do not expect all technical work to cease by the end of 2012. The phase two plan of the Superconducting Radiofrequency Test Facility at KEK is scheduled to run to (at earliest) 2014. The Fermilab-based string test is completed in 2012 but routine operations would only start in 2013 and would continue for several years at least. Cryomodule and cavity value engineering will remain a highly leveraged item and it is reasonable to assume that positron production will remain a topic of interest. The Super-KEKB (2013) will incorporate several of the CesrTA electron cloud-mitigation techniques. The anticipated technical programme for the subsequent several years will be described in the PIP.

This interim report focuses mainly on the ILC R\&D progress and accomplishments since the Reference Design Report was published. It marks a halfway point toward completion of the ILC Technical Design Phase, which will be documented at the end of 2012 in a Technical Design Report. The TDR will be a complete report that contains all that will be needed to propose the ILC to collaborating governments, including the technical design, costing and an implementation plan. The design has evolved significantly since the RDR, reflecting a more optimised and coherent design that balances cost, risk and performance. In addition, by the end of 2012 the key R\&D demonstrations will have been completed and the project will be ready to be proposed at any time after the TDR.

The GDE will have accomplished its mandate once the TDR is published, reviewed and accepted by our oversight committees (around mid-2013). Nevertheless, there will still be important R\&D still to be continued, and improvements in the design will undoubtedly result. In fact, the ILC design will continue to evolve until the project is approved for construction.

Guidance from LHC results is very much sought. The first extended LHC run will take place during the coming two years, during TDR completion. The most significant results are expected during the post-2012 period. The LHC physics results will both sharpen the physics motivation and help guide the choice of machine parameters, including the initial energy. The ILC design must remain flexible enough to respond to this emerging physics. At present, the biggest unknown is determining what energy a lepton collider will be required to match LHC physics. If the ILC reach of about $1 \mathrm{TeV}$ is a good match, then the ILC is clearly the right machine to propose based on maturity of the technology and achievable physics parameters. However, if much higher energy is required, other approaches like CLIC or a muon collider will be required; both will require more R\&D before a project can be proposed.

\subsection{BEYOND THE TECHNICAL DESIGN REPORT}


After the TDR, the highest priority will continue to be developing highgradient SCRF through research that may substantially increase the gradient, such as developing different cavity shapes. On a systems level, the Superconducting Radiofrequency Test Facility programmes at KEK and the superconducting radiofrequency test accelerator in the New Muon Lab at Fermilab will just be coming to fruition and a several-year programme of research and testing will follow. There will also be continuing R\&D in other areas, including positron source R\&D, final focus tests at ATF2, assuming the ATF continues to run, and other endeavours. Perhaps the largest unfinished task that has to be completed is a value engineering study, after which the final engineering design can proceed and the construction begun.

It will be possible to carry out the post-TDR programme at a somewhat reduced support level compared to the present programme, but it must be done in a fashion poised to ramp up as soon as commitments are made for an ILC construction project. The post-TDR R\&D programme will be carried out by a successor organisation to the GDE; the ILC Steering Committee and International Committee for Future Accelerators are in discussions as to how to structure such a new organisation. The GDE management is providing input for that process. All agree that whatever successor organisation is created there will need to be continuity of key personnel and core expertise, as well as the ability to maintain a global process for setting priorities and making decisions.

Although the future of the ILC R\&D programme following the TDR is presently unclear, we remain ready to submit a very strong construction proposal whenever governments are receptive to considering a future large global project in particle physics.

References

[6-1] TESLA proposal.

[6-2] http://ilcagenda.linearcollider.org/

conferenceDisplay. py? confld=4530 
TOWARDS THE TECHNICAL DESIGN REPORT 


\section{AUTHOR} LIST
Ned Arnold ${ }^{1}$, Sebastian Aderhold ${ }^{16}$, Chris Adolphsen ${ }^{44}$, Robert

Ainsworth $^{28}$, Yasuo Ajima ${ }^{31}$, Mituo Akemoto $^{31}$, Javier Alabau ${ }^{21}$, Maria Alabau Pons ${ }^{9}$, David Alesini ${ }^{24}$, James Alexander ${ }^{15}$, John Amann ${ }^{44}$, Deepa Angal-Kalinin ${ }^{45}$, Robert Apsimon $^{28}$, Sakae Araki ${ }^{31}$, Tug Arkan $^{18}$, Alexander Aryshev ${ }^{31}$, David Asner $^{7}$, Valeri Ayvazyan ${ }^{16}$, Nicoleta $\mathrm{Baboi}^{16}$, Sha Bai ${ }^{22}$, Ian Bailey ${ }^{34}$, Philip Bambade $^{11}$, Serena Barbanotti ${ }^{18}$, Barry Barish $^{5}$, Marco Battistoni ${ }^{18}$, Victoria Bayliss ${ }^{46}$, Paul Bellomo ${ }^{44}$, Douglas Bett ${ }^{28}$, Wilhelm Bialowons ${ }^{16}$, Michael Billing ${ }^{15}$, Grahame Blair ${ }^{28}$, Cesar Blanch ${ }^{21}$, Benoit Bolzon ${ }^{9}$, Stewart Boogert ${ }^{28}$, Laura Boon ${ }^{42}$, Gary Boorman ${ }^{28}$, Angelo Bosotti ${ }^{23}$, Axel Brachmann ${ }^{44}$, Tom Bradshaw ${ }^{46}$, Amanda Brummitt ${ }^{46}$, Yulian Budagov $^{26}$, Benjamin Bullock ${ }^{15}$, Craig Burkhart ${ }^{44}$, Philip Burrows ${ }^{28}$, Geoff Burton ${ }^{46}$, John Byrd ${ }^{36}$, Sergio Calatroni ${ }^{9}$, Joseph Calvey ${ }^{15}$, Simon Canfer ${ }^{46}$, Steve Carr ${ }^{46}$, Paul Carriere $^{15}$, Harry Carter ${ }^{18}$, John Carwardine $^{1}$, Christine Celata ${ }^{36}$, Mark Champion ${ }^{18}$, Stephane $\mathrm{Chel}^{8}$, Jia-er Chen ${ }^{41}$, Glenn Christian ${ }^{28}$, Wojciech Cichalewski ${ }^{16}$, Jim Clarke ${ }^{45}$, Paul Coe ${ }^{28}$, Norbert Collomb ${ }^{45}$, Ben Constance $^{28}$, Zachary Conway ${ }^{15}$, Lance Cooley ${ }^{18}$, Charlie Cooper ${ }^{18}$, Laura Corner ${ }^{29}$, Mike Courthold ${ }^{46}$, Anthony Curtis Crawford ${ }^{15}$, James Crittenden $^{15}$, Francis Cullinan ${ }^{28}$, T.S. Datta $^{25}$, Michael Davis ${ }^{28}$, Stefano De Santis ${ }^{36}$, Lawrence Deacon ${ }^{28}$,
Jean-Pierre Delahaye ${ }^{9}$, Theo Demma $^{24}$, Yury Denisov ${ }^{26}$, Andrey Dudarev $^{26}$, Gerald Dugan ${ }^{15}$, Nicholas Eggert $^{15}$, George Ellwood ${ }^{46}$, Eckhard Elsen $^{16}$, Atsushi Enomoto ${ }^{31}$, Fabien Eozenou $^{8}$, Grigory Eremeev ${ }^{27}$, Bart Faat ${ }^{16}$, Angeles Faus-Golfe ${ }^{21}$, John Flanagan ${ }^{31}$, Mike Foley ${ }^{18}$, Michael Forster ${ }^{15}$, Josef Frisch ${ }^{44}$, Lars Froehlich $^{16}$, Shigeki Fukuda ${ }^{31}$, Masafumi Fukuda ${ }^{31}$, Yoshisato Funahashi ${ }^{31}$, Miguel Furman ${ }^{36}$, Fumio Furuta ${ }^{31}$, Wei Gai ${ }^{1}$, Jie Gao ${ }^{22}$, Mingqi Ge ${ }^{15}$, Nicolas Geffroy ${ }^{12}$, Rongli Geng $^{27}$, Eliana Gianfelice-Wendt ${ }^{18}$, Camille Ginsburg ${ }^{18}$, Mariusz Grecki $^{16}$, Shlomo Greenwald ${ }^{15}$, Jeffrey Gronberg ${ }^{37}$, Susanna Guiducci ${ }^{24}$, Hayg Guler ${ }^{38}$, Kazufumi Hara ${ }^{31}$, Katherine Harkay ${ }^{1}$, Elvin Harms ${ }^{18}$, Mike Harrison ${ }^{4}$, Donald Hartill ${ }^{15}$, Hitoshi Hayano ${ }^{31}$, Olaf Hensler ${ }^{16}$, Aeyoung $\mathrm{Heo}^{33}$, Leah Hesla ${ }^{18}$, Stefan Hesselbach $^{17}$, Norio Higashi ${ }^{31}$, Matthew Hills ${ }^{46}$, Andy Hocker ${ }^{18}$, Georg H. Hoffstaetter ${ }^{15}$, Robert Holtzapple ${ }^{6}$, Yosuke Honda ${ }^{31}$, Katja Honkavaara $^{16}$, Walter Hopkins ${ }^{15}$, Kenji Hosoyama ${ }^{31}$, Jung-Yun Huang $^{40}$, Andrew Hutton ${ }^{27}$, Woon-Ha Hwang ${ }^{40}$, Alexander Ignatenko ${ }^{16}$, Hitoshi Inoue ${ }^{31}$, Yoshihisa Iwashita ${ }^{32}$, Wojciech Jamuzna $^{16}$, Michael Jenkins ${ }^{34}$, Kay Jensch $^{16}$, Andréa Jeremie ${ }^{12}$, James Jones $^{45}$, Nirav Joshi ${ }^{28}$, C.K. Joshi ${ }^{43}$, Eiji Kako ${ }^{31}$, Yoshio Kamiya ${ }^{48}$, Raimund Kammering ${ }^{16}$, Ken-ichi Kanazawa $^{31}$, Pavel Karataev ${ }^{28}$,

$\begin{array}{llll} & & \\ & & \\ \text { ANL } & { }^{7} \text { Carleton University } & { }^{14} \text { Cockcroft Institute/ } & { }^{21} \text { IFIC (CSIC-UV) } \\ { }^{2} \text { Bhabha Atomic } & { }^{8} \text { CEA/Irfu } & \text { University of } & { }^{22} \text { IHEP } \\ \text { Research Centre } & { }^{9} \text { CERN } & \text { Liverpool } & { }^{23} \text { INFN Milano } \\ { }^{3} \text { BINP Novosibirsk } & { }^{10} \text { CNRS/IN2P3 } & { }^{15} \text { Cornell University } & { }^{24} \text { INFN-LNF } \\ { }^{4} \text { BNL } & { }^{11} \text { CNRS/LAL } & { }^{16} \text { DESY } & { }^{25} \text { IUAC } \\ { }^{5} \text { California Institute of } & { }^{12} \text { CNRS/LAPP } & { }^{17} \text { Durham University } & { }^{26} \text { JINR } \\ \text { Technology } & { }^{13} \text { CNRS/LLR } & { }^{18} \text { Fermilab } & { }^{27} \text { JLab } \\ { }^{6} \text { California Polytechnic } & & { }^{19} \text { Hamburg University } & \\ \text { State University } & & { }^{20} \text { Hiroshima University } & \end{array}$


Vladimir Kashikhin ${ }^{18}$, Hiroaki Katagiri $^{31}$, Valery Katalev ${ }^{16}$, Shigeki Kato $^{31}$, Michael P. Kelly ${ }^{1}$, Robert Kephart ${ }^{18}$, Jim Kerby ${ }^{18}$, Timergali Khabiboulline $^{18}$, Jin-Sung Kim ${ }^{15}$, EunSan Kim³ ${ }^{33}$, HyoungSuk Kim³, SeungHwan Kim³ ${ }^{33}$ Arkadiy Klebaner $^{18}$, Peter Kneisel ${ }^{27}$, Yuji Kojima $^{31}$, Sachio Komamiya ${ }^{48}$, Yoshinari Kondo ${ }^{31}$, Waldemar Koprek $^{16}$, Denis Kostin ${ }^{16}$, Anatoly Krasnykh ${ }^{44}$, David Kreinick ${ }^{15}$, Benjamin Kreis ${ }^{15}$, Dirk Kruecker ${ }^{16}$, Kiyoshi Kubo ${ }^{31}$, Vic Kuchler ${ }^{18}$, Kiran Kulkarni², Tatsuya Kume ${ }^{31}$, Shigeru Kuroda ${ }^{31}$, Mickaël Lacroix ${ }^{11}$, Briant Lam ${ }^{44}$, Andrea Latina ${ }^{9}$, Jerry Leibfritz $^{18}$, Konstantin Lekomtsev ${ }^{28}$, Yulin $\mathrm{Li}^{15}$, Lutz Lilje ${ }^{16}$, Wanming $\mathrm{Liu}^{1}$, Xianghong Liu ${ }^{15}$, Kexin Liu ${ }^{41}$, Jesse Livezey ${ }^{15}$, Florian Loehl ${ }^{15}$, J. Lucas ${ }^{45}$, Alexey Lyapin ${ }^{28}$, Oleg Malyshev $^{45}$, Fabio Marcellini' ${ }^{24}$, Mika Masuzawa $^{31}$, Axel Matheisen ${ }^{16}$, Toshihiro Matsumoto ${ }^{31}$, Douglas McCormick $^{44}$, Robert Meller ${ }^{15}$, Shinichiro Michizono ${ }^{31}$, Alexander Mikhailichenko $^{15}$, Shekhar Mishra ${ }^{18}$, Don Mitchell ${ }^{18}$, Takako Miura ${ }^{31}$, Wolf-Dietrich Moeller ${ }^{16}$, Stephen Molloy $^{28}$, Gudrid Moortgat-Pick ${ }^{19}$, Dawn Munson ${ }^{36}$, Sergei Nagaitsev ${ }^{18}$, Takashi Naito ${ }^{31}$, Hirotaka Nakai ${ }^{31}$, Hiromitsu Nakajima ${ }^{31}$, Tomoaki Nakamura $^{48}$, Chris Nantista ${ }^{44}$, Olivier Napoly $^{8}$, Janice Nelson ${ }^{44}$, Laurence Nevay $^{29}$, Shuichi Noguchi ${ }^{31}$, Kazuhito Ohmi $^{31}$, Norihito Ohuchi ${ }^{31}$, Daisuke Okamoto $^{47}$, Toshiyuki Okugi ${ }^{31}$,
Masahiro Oroku ${ }^{48}$, John Osborne ${ }^{9}$, Joe Ozelis $^{18}$, Hasan Padamsee ${ }^{15}$, Carlo Pagani $^{23}$, Mark Palmer ${ }^{15}$, Yannis Papaphilippou ${ }^{9}$, Rocco Paparella ${ }^{23}$, Yong Jung Park ${ }^{40}$, Brett Parker ${ }^{4}$, Ewan Paterson ${ }^{4}$, Jacques Payet ${ }^{8}$, Shilun $\mathrm{Pei}^{44}$, Gregg Penn ${ }^{36}$, Colin Perry ${ }^{28}$, Daniel Peterson ${ }^{15}$, Tom Peterson ${ }^{18}$, Paulo Pierini ${ }^{23}$, Tom Piggott ${ }^{37}$, Yuriy Pischalnikov ${ }^{18}$, Mauro Pivi ${ }^{44}$, Freddy Poirier ${ }^{16}$, S. Postlethwaite ${ }^{45}$, Eduard Pratt ${ }^{16}$, Pravin Rai ${ }^{2}$, Pantaleo Raimondi $^{24}$, Tor Raubenheimer ${ }^{44}$, Charles Reece ${ }^{27}$, Kay Rehlich ${ }^{16}$, Yves Renier ${ }^{9}$, Javier Resta Lopez ${ }^{29}$, Nathan Rider $^{15}$, Sabine Riemann ${ }^{16}$, Cécile Rimbault $^{11}$, Bob Rimmer ${ }^{27}$, Louis Rinolfi $^{9}$, James Rochford ${ }^{46}$, Marc Ross $^{18}$, Allan Rowe ${ }^{18}$, Amit Roy ${ }^{25}$, Perrine Royole-Degieux ${ }^{10}$, David Rubin $^{15}$, Giovanni Rumolo9, Takayuki Saeki $^{31}$, David Sagan ${ }^{15}$, Kenji Saito ${ }^{31}$, Tomoyuki Sanuki ${ }^{47}$, Masato Sato ${ }^{31}$, Polepalle Styamurthy², Motoaki Sawabi $^{31}$, Anthony Scarfe ${ }^{39}$, Andreas Schaelicke ${ }^{16}$, Joern Schaffran ${ }^{16}$, Warren Schappert ${ }^{18}$, P. Schilling ${ }^{16}$, Felix Schlander ${ }^{16}$, Holger Schlarb ${ }^{16}$, Christian Schmidt ${ }^{16}$, M. Schmoekel ${ }^{16}$, Siegfried Schreiber ${ }^{16}$, Daniel Schulte ${ }^{9}$, Duncan Scott ${ }^{45}$, Andrei Seryi ${ }^{30}$, James Shanks $^{15}$, Valery Shemelin ${ }^{15}$, Kyo Shibata $^{31}$, Tetsuo Shidara ${ }^{31}$, Gregory Shirkov $^{26}$, Toshiro Shishido ${ }^{31}$, John Sikora $^{15}$, Stefan Simrock ${ }^{16}$, Waldemar Singer $^{16}$, Brian Smith ${ }^{18}$, Steve Smith ${ }^{44}$, Tonee Smith ${ }^{44}$, Nikolay Solyak ${ }^{18}$, Kiran Sonnad ${ }^{15}$, Cherrill Spencer ${ }^{44}$, Martin Staack ${ }^{16}$, Rich Stanek ${ }^{18}$,
Taikan Suehara ${ }^{31}$, Yusuke Suetsugu ${ }^{31}$, Ryuhei Sugahara ${ }^{31}$, Christina Swinson $^{29}$, Cosmore Sylvester ${ }^{18}$, Jaroslaw Szewinski ${ }^{16}$, Mauro Taborelli $^{9}$, Tsuyoshi Tajima ${ }^{35}$, Tohru Takahashi $^{20}$, Rika Takahashi ${ }^{31}$, Tateru Takenaka $^{31}$, Tao Tang ${ }^{44}$, Toshiaki Tauchi $^{31}$, Owen Taylor ${ }^{46}$, Akio Terashima $^{31}$, Nobuhiro Terunuma ${ }^{31}$, Jeff Tice ${ }^{44}$, Vikas Tiwari², Nobukazu Toge $^{31}$, Rogelio Tomas 9 , Grigori Trubnikov ${ }^{26}$, Kiyosumi Tsuchiya ${ }^{31}$, Kenji Ueno ${ }^{31}$, Junji Urakawa ${ }^{31}$, David Urner ${ }^{29}$, Andrei Ushakov ${ }^{16}$, Alessandro Variola ${ }^{11}$, Marco Venturini $^{3^{6}}$, Marc Verderi ${ }^{13}$, Reine Versteegen $^{8}$, Bernard Visentin ${ }^{8}$, Nicholas Walker $^{16}$, Dou Wang ${ }^{22}$, Lanfa Wang44, Min-Huey Wang44, Faya Wang ${ }^{44}$, Matthew Warden ${ }^{29}$, Barbara Warmbein $^{16}$, Ken Watanabe ${ }^{31}$, Yuichi Watanabe $^{31}$, Stephen Watson ${ }^{46}$, Hans Weise $^{16}$, Manfred Wendt ${ }^{18}$, Glen White ${ }^{44}$, Tim Wilksen ${ }^{16}$, Ingo Will ${ }^{49}$, Kay Wittenburg ${ }^{16}$, Walter Wittmer ${ }^{44}$, Torsten Wohlenberg ${ }^{16}$, Andrzej Wolski $^{14}$, Mayling Wong-Squires ${ }^{18}$, Mark Woodley ${ }^{44}$, Mike Woodward ${ }^{46}$, Seiya Yamaguchi ${ }^{31}$, Youhei Yamaguchi $^{48}$, Akira Yamamoto ${ }^{31}$, Yasuchika Yamamoto ${ }^{31}$, Takashi Yamanaka ${ }^{48}$, Yiton Yan ${ }^{44}$, Jacqueline Yan $^{48}$, Hakutaro Yoda ${ }^{48}$, Kaoru Yokoya $^{31}$, Jiyuan Zhai ${ }^{22}$, Min Zhang ${ }^{22}$, Feng Zhou ${ }^{44}$, Frank Zimmerman", Konstantin Zolotarev ${ }^{3}$.

\begin{tabular}{|c|c|c|c|c|}
\hline${ }^{28}$ John Adams Institute & ${ }^{30}$ John Adams Institute & ${ }^{33}$ Kyungpook National & ${ }^{41} \mathrm{PKU}$ & ${ }^{48}$ Tokyo University \\
\hline for Accelerator & for Accelerator & University & ${ }^{42}$ Purdue University & ${ }^{49}$ TU Berlin \\
\hline Science and Royal & Science, University & ${ }^{34}$ Lancaster University & ${ }^{43}$ RRCAT & \\
\hline Holloway, University & of Oxford and Royal & ${ }^{35}$ LANL & ${ }^{44}$ SLAC & \\
\hline of London & Holloway, University & ${ }^{36}$ LBNL & ${ }^{45}$ STFC, Daresbury & \\
\hline${ }^{29}$ John Adams Institute & of London & ${ }^{37}$ LLNL & Laboratory & \\
\hline for Accelerator & ${ }^{31} \mathrm{KEK}$ & ${ }^{38} \mathrm{LLRL}$ & ${ }^{46}$ STFC, Rutherford & \\
\hline Science and & ${ }^{32}$ Kyoto University & ${ }^{39}$ Manchester University & Appleton Laboratory & \\
\hline University of Oxford & & ${ }^{40} \mathrm{PAL}$ & ${ }^{47}$ Tohoku University & \\
\hline
\end{tabular}




\section{in \\ stovion international linear collider}

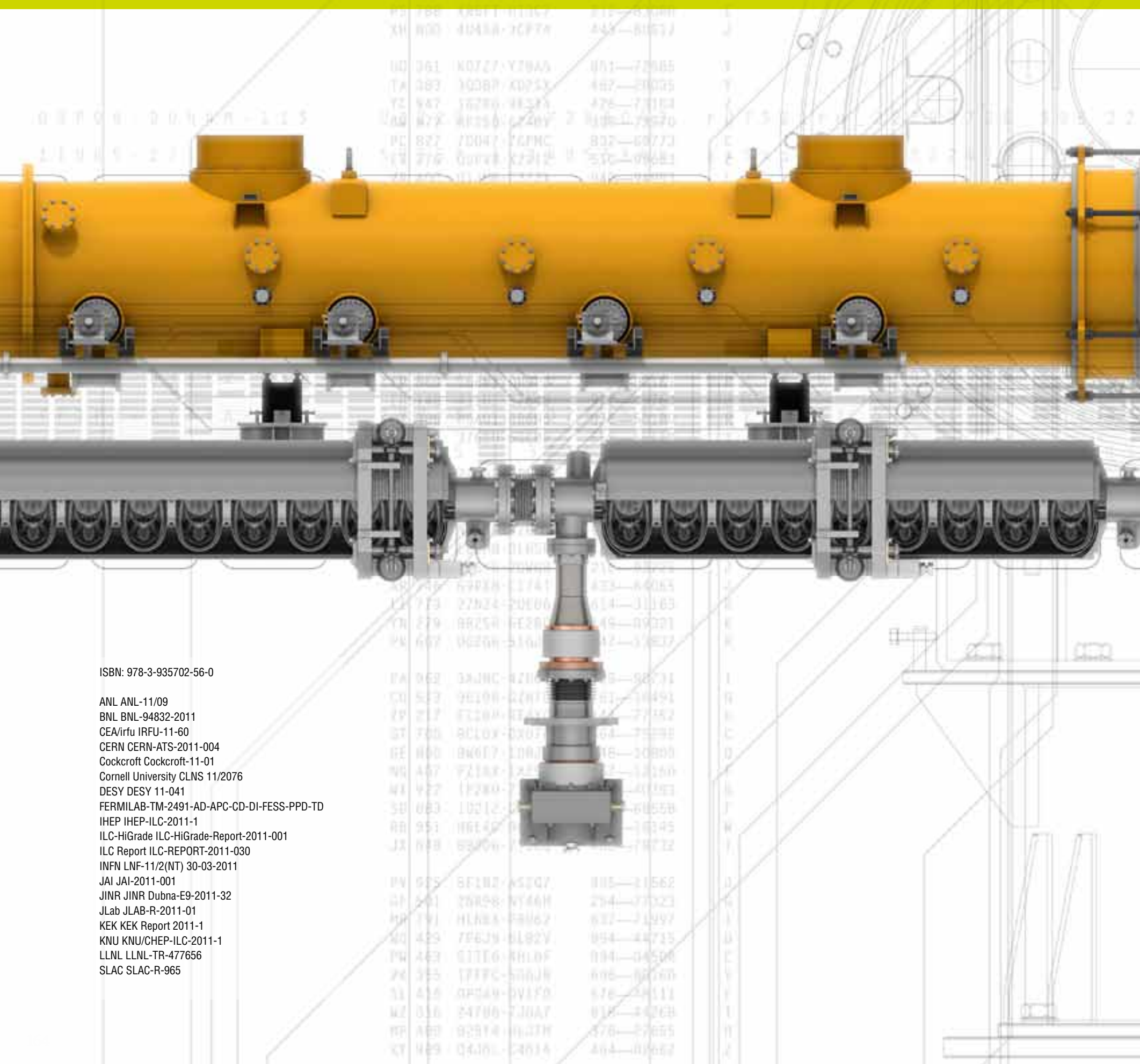




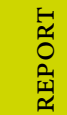

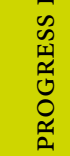

茯 